\title{
KRAGUJEVAC JOURNAL OF MATHEMATICS
}

Volume 46, Number 1, 2022

University of Kragujevac

Faculty of Science 
CIP - Каталогизација у публикацији

Народна библиотека Србије, Београд

51

KRAGUJEVAC Journal of Mathematics / Faculty of Science, University of Kragujevac ; editor-in-chief Suzana Aleksić

. - Vol. 22 (2000)- . - Kragujevac : Faculty of Science, University

of Kragujevac, 2000- (Belgrade : Donat Graf). - $24 \mathrm{~cm}$

Dvomesečno. - Delimično je nastavak: Zbornik radova Prirodnomatematičkog fakulteta (Kragujevac) = ISSN 0351-6962. - Drugo izdanje na drugom medijumu: Kragujevac Journal of Mathematics (Online) $=$ ISSN 2406-3045

ISSN 1450-9628 = Kragujevac Journal of Mathematics COBISS.SR-ID 75159042

DOI 10.46793/KgJMat2201

Published By: $\quad$ Faculty of Science University of Kragujevac Radoja Domanovića 12 34000 Kragujevac Serbia

Tel.: +381 (0)34 336223

Fax: +381 (0)34335040

Email: krag_j_math@kg.ac.rs

Website: http://kjm.pmf.kg.ac.rs

Designed By: Thomas Lampert

Front Cover: $\quad$ Željko Mališić

Printed By: Donat Graf, Belgrade, Serbia

From 2021 the journal appears in one volume and six issues per annum. 


\section{Editor-in-Chief:}

- Suzana Aleksić, University of Kragujevac, Faculty of Science, Kragujevac, Serbia

\section{Associate Editors:}

- Tatjana Aleksić Lampert, University of Kragujevac, Faculty of Science, Kragujevac, Serbia

- Đorđe Baralić, Mathematical Institute of the Serbian Academy of Sciences and Arts, Belgrade, Serbia

- Dejan Bojović, University of Kragujevac, Faculty of Science, Kragujevac, Serbia

- Bojana Borovićanin, University of Kragujevac, Faculty of Science, Kragujevac, Serbia

- Nada Damljanović, University of Kragujevac, Faculty of Technical Sciences, Čačak, Serbia

- Jelena Ignjatović, University of Niš, Faculty of Natural Sciences and Mathematics, Niš, Serbia

- Nebojša Ikodinović, University of Belgrade, Faculty of Mathematics, Belgrade, Serbia

- Boško Jovanović, University of Belgrade, Faculty of Mathematics, Belgrade, Serbia

- Marijan Marković, University of Montenegro, Faculty of Science and Mathematics, Podgorica, Montenegro

- Emilija Nešović, University of Kragujevac, Faculty of Science, Kragujevac, Serbia

- Marko Petković, University of Niš, Faculty of Natural Sciences and Mathematics, Niš, Serbia

- Marija Stanić, University of Kragujevac, Faculty of Science, Kragujevac, Serbia

\section{Editorial Board:}

- Ravi P. Agarwal, Department of Mathematics, Texas A\&M University-Kingsville, Kingsville, TX, USA

- Dragić Banković, University of Kragujevac, Faculty of Science, Kragujevac, Serbia

- Richard A. Brualdi, University of Wisconsin-Madison, Mathematics Department, Madison, Wisconsin, USA

- Bang-Yen Chen, Michigan State University, Department of Mathematics, Michigan, USA

- Claudio Cuevas, Federal University of Pernambuco, Department of Mathematics, Recife, Brazil

- Miroslav Ćirić, University of Niš, Faculty of Natural Sciences and Mathematics, Niš, Serbia

- Sever Dragomir, Victoria University, School of Engineering \& Science, Melbourne, Australia

- Vladimir Dragović, The University of Texas at Dallas, School of Natural Sciences and Mathematics, Dallas, Texas, USA and Mathematical Institute of the Serbian Academy of Sciences and Arts, Belgrade, Serbia 
- Paul Embrechts, ETH Zurich, Department of Mathematics, Zurich, Switzerland

- Ivan Gutman, University of Kragujevac, Faculty of Science, Kragujevac, Serbia

- Mircea Ivan, Technical University of Cluj-Napoca, Department of Mathematics, Cluj- Napoca, Romania

- Sandi Klavžar, University of Ljubljana, Faculty of Mathematics and Physics, Ljubljana, Slovenia

- Giuseppe Mastroianni, University of Basilicata, Department of Mathematics, Informatics and Economics, Potenza, Italy

- Miodrag Mateljević, University of Belgrade, Faculty of Mathematics, Belgrade, Serbia

- Gradimir Milovanović, Serbian Academy of Sciences and Arts, Belgrade, Serbia

- Sotirios Notaris, National and Kapodistrian University of Athens, Department of Mathematics, Athens, Greece

- Miroslava Petrović-Torgašev, University of Kragujevac, Faculty of Science, Kragujevac, Serbia

- Stevan Pilipović, University of Novi Sad, Faculty of Sciences, Novi Sad, Serbia

- Juan Rada, University of Antioquia, Institute of Mathematics, Medellin, Colombia

- Stojan Radenović, University of Belgrade, Faculty of Mechanical Engineering, Belgrade, Serbia

- Lothar Reichel, Kent State University, Department of Mathematical Sciences, Kent $(\mathrm{OH}), \mathrm{USA}$

- Miodrag Spalević, University of Belgrade, Faculty of Mechanical Engineering, Belgrade, Serbia

- Hari Mohan Srivastava, University of Victoria, Department of Mathematics and Statistics, Victoria, British Columbia, Canada

- Kostadin Trenčevski, Ss Cyril and Methodius University, Faculty of Natural Sciences and Mathematics, Skopje, Macedonia

- Boban Veličković, University of Paris 7, Department of Mathematics, Paris, France

- Leopold Verstraelen, Katholieke Universiteit Leuven, Department of Mathematics, Leuven, Belgium

\section{Technical Editor:}

- Tatjana Tomović Mladenović, University of Kragujevac, Faculty of Science, Kragujevac, Serbia 


\section{Contents}

K. Ali Khelil New Integral Equations for the Monic Hermite Polynomials7

R. Sfaxi

A. Boukhemis

F. Ali

Connectedness of the Cut System Complex on Nonorientable

F. Atalan

Surfaces ....................................21

M. B. Esfahani $\quad w M B$-Property of Order $p$ in Banach Spaces............29

F. Sultanzadeh Refining some Inequalities for Frames with Specht's Ratio39

M. Hassani

M. E. Omidvar

R. A. K. Gol

M. Beddani

Solution Set for Impulsive Fractional Differential Inclusions................................ 49

M. M. Woldaregay Uniformly Convergent Numerical Method for Singularly Per-

G. F. Duressa

turbed Delay Parabolic Differential Equations Arising in Computational Neuroscience ........................6 65

A. Zada

Existence and Stability Analysis of Sequential Coupled Sys-

M. Yar tem of Hadamard-Type Fractional Differential Equations 85

R. Cruz

Extremal Graphs for Exponential VDB Indices 105

J. Rada

A. K. Wanas

Differential Subordination Results for Holomorphic Functions Related to Generalized Differential Operator........... 115

A. Rezaei Pseudo-BCK Algebras Derived from Directoids 125

S. Hans

A Note on Comparison of Annuli Containing all the Zeros of

A. Tomar a Polynomial...

J. Chen

V. Gupta

Some Results on Post-Widder Operators Preserving Test

G. Tachev

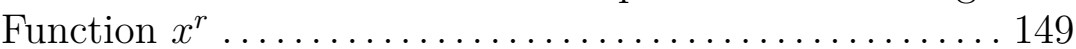



Kragujevac Journal of Mathematics

Volume 46(1) (2022), Pages 7-19.

\title{
NEW INTEGRAL EQUATIONS FOR THE MONIC HERMITE POLYNOMIALS
}

\author{
KARIMA ALI KHELIL ${ }^{1}$, RIDHA SFAXI ${ }^{2}$, AND AMMAR BOUKHEMIS ${ }^{1}$
}

\begin{abstract}
In this article, we are study the question of existence of integral equation for the monic $\mathcal{H}$ ermite polynomials $H_{n}$, where the intervening real function does not depend on the index $n$, well-known by the linear functional $\mathscr{W}_{x}$ given by its moments $H_{n}(x)=\left\langle\mathscr{W}_{x}, t^{n}\right\rangle, n \geq 0,|x|<\infty$. Also, we obtain some properties of the zeros of this intervening function. Furthermore, we obtain an integral representation of the Dirac mass $\delta_{x}$, for every real number $x$.
\end{abstract}

\section{INTRODUCTION}

Given two sequences $\left\{B_{n}\right\}_{n \geq 0}$ and $\left\{Q_{n}\right\}_{n \geq 0}$ of normalized polynomials with real coefficients, with one real variable $x$ and where $\operatorname{deg} B_{n}=\operatorname{deg} Q_{n}=n$, for every integer $n \geq 0$. The problem of integral equation between these two polynomial sequences consists in finding a real function $u(\cdot, t)$ defined in $I \times \mathbb{R}$, where $I \subset \mathbb{R}=]-\infty,+\infty$, and satisfying the condition:

$$
\int_{-\infty}^{\infty} u(x, t) t^{n} \mathrm{~d} t<\infty, \quad n \geq 0, x \in I
$$

such that

$$
B_{n}(x)=\int_{-\infty}^{\infty} u(x, t) Q_{n}(t) \mathrm{d} t, \quad n \geq 0, x \in I .
$$

When $Q_{n}(x)=x^{n}$, for all integer $n \geq 0$, i.e.,

$$
B_{n}(x)=\int_{-\infty}^{\infty} u(x, t) t^{n} \mathrm{~d} t, \quad n \geq 0, x \in I,
$$

Key words and phrases. Linear functional, integral equation, integral representation on the real line, Hermite polynomials, Dawson function, Dirac mass.

2010 Mathematics Subject Classification. Primary: 33C45, 42C05.

DOI 10.46793/KgJMat2201.007K

Received: January 19, 2019.

Accepted: July 25, 2019. 
we recognize the usual integral representation of the polynomial sequence $\left\{B_{n}\right\}_{n \geq 0}$, called here by the canonical-integral representation of $\left\{B_{n}\right\}_{n \geq 0}$. When $Q_{n}(x)=B_{n}(x)$, for all integer $n \geq 0$, i.e.,

$$
B_{n}(x)=\int_{-\infty}^{\infty} u(x, t) B_{n}(t) \mathrm{d} t, \quad n \geq 0, x \in I,
$$

it is appropriate to say that it is an auto-integral representation of $\left\{B_{n}\right\}_{n \geq 0}$.

In fact, this kind of integral equation is of great relevance in the theory of orthogonal polynomials as well as the moment theory and their applications, [8,9,3,15]. For this reason-in the past as nowadays has attracted the attention of many authors; see, for instance, $[5,6,7,12,4,1,10,11]$. Based on the principle that the terms of any sequence of complex numbers are the moments of a unique linear functional on polynomials, the study of such linear functionals accurate some hypergeometric properties of such sequences, $[2,13,14]$.

In this work, we are interested by the normalized Hermite polynomial sequence $\left\{H_{n}\right\}_{n \geq 0}$. Recall that $\left\{H_{n}\right\}_{n \geq 0}$ is orthogonal with respect to a linear functional on polynomials, namely $\mathscr{H}$ and well-known by its integral representation on the real line $[10]$

$$
\langle\mathscr{H}, p\rangle=\frac{1}{\sqrt{\pi}} \int_{-\infty}^{\infty} p(t) e^{-t^{2}} \mathrm{~d} t, \quad p \in \mathbb{P},
$$

where $\mathbb{P}$ is the vector space of polynomials in one variable with real coefficients and $\mathbb{P}^{\prime}$ its algebraic dual space. Notice that $\langle u, p\rangle$ is the action of a linear functional $u \in \mathbb{P}^{\prime}$ on $p \in \mathbb{P}$ and by $(u)_{n}:=\left\langle u, t^{n}\right\rangle, n \geq 0$, the moments of $u$ with respect to the canonical sequence $\left\{t^{n}\right\}_{n>0}$. For any $u$ in $\mathbb{P}^{\prime}$, any $q$ in $\mathbb{P}$ and any complex numbers $a, b, c$ with $a \neq 0$, recall that $D u=u^{\prime}, q u, h_{a} u$ and $\tau_{b} u$, be respectively, the derivative, the left multiplication, the homothetic and the translation of the linear functionals defined by duality [9]:

$$
\begin{aligned}
\left\langle u^{\prime}, f\right\rangle & :=-\left\langle u, f^{\prime}\right\rangle, \\
\langle q u, f\rangle & :=\langle u, q f\rangle \\
\left\langle h_{a} u, f\right\rangle & :=\left\langle u, h_{a} f\right\rangle=\langle u, f(a x)\rangle, \\
\left\langle\tau_{-b} u, f\right\rangle & :=\left\langle u, \tau_{b} f\right\rangle=\langle u, f(x-b)\rangle, \quad f \in \mathbb{P} .
\end{aligned}
$$

The linear functional $\mathscr{H}$ is normalized, i.e., $(\mathscr{H})_{0}=1$. It satisfies the following Pearson equation [10]:

$$
\mathscr{H}^{\prime}+2 x \mathscr{H}=0_{\mathbb{P}^{\prime}} .
$$

The moments of $\mathscr{H}$ are given by

$$
(\mathscr{H})_{n}=\frac{n !}{2^{n+1} \Gamma\left(\frac{n}{2}+1\right)}\left(1+(-1)^{n}\right), \quad n \geq 0 .
$$

This leads to the following integral representation of the moments of $\mathscr{H}$

$$
\frac{n !}{2^{n+1} \Gamma\left(\frac{n}{2}+1\right)}\left(1+(-1)^{n}\right)=\frac{1}{\sqrt{\pi}} \int_{-\infty}^{\infty} t^{n} e^{-t^{2}} \mathrm{~d} t, \quad n \geq 0 .
$$


The normalized Hermite polynomial $H_{n}$ can be represented in terms of a definite integral containing the real variable $x$ as parameter [8]

$$
H_{n}(x)=\frac{e^{x^{2}}}{\sqrt{\pi}} \int_{-\infty}^{\infty}(-i t)^{n} e^{-t^{2}+2 i t x} \mathrm{~d} t, \quad n \geq 0,|x|<\infty .
$$

Equivalently,

$$
H_{n}(x)=\int_{0}^{\infty} h_{n}(t, x) t^{n} \mathrm{~d} t, \quad n \geq 0,|x|<\infty
$$

where the intervening real function $h_{n}(t, \cdot)$ depends on the integer $n$, and given by

$$
h_{n}(t, x)=\frac{2}{\sqrt{\pi}} e^{x^{2}-t^{2}} \cos \left(2 t x+n \frac{\pi}{2}\right) .
$$

The polynomial $H_{n}$ satisfies the following integral equation [8]

$$
H_{n}(x)=\frac{(-i)^{n}}{\sqrt{2 \pi}} e^{\frac{x^{2}}{2}} \int_{-\infty}^{\infty} e^{-\frac{t^{2}}{2}+i t x} H_{n}(t) \mathrm{d} t, \quad n \geq 0,|x|<\infty .
$$

Equivalently,

$$
H_{n}(x)=\int_{0}^{\infty} r_{n}(t, x) H_{n}(t) \mathrm{d} t, \quad n \geq 0,|x|<\infty,
$$

where the real function $r_{n}(t, \cdot)$ depends on the integer $n$, and given by

$$
r_{n}(t, x)=\frac{1}{\sqrt{2}} h_{n}\left(\frac{t}{\sqrt{2}}, \frac{x}{\sqrt{2}}\right) .
$$

The main purpose of this work is to give two new integral equations for the polynomial sequence $\left\{H_{n}\right\}_{n \geq 0}$, where the intervening real functions do not depend on the integer $n$. In summary, we are going to establish the following.

- The canonical-integral representation:

$$
H_{n}(x)=\int_{-\infty}^{\infty} U(t-x) t^{n} \mathrm{~d} t, \quad n \geq 0,|x|<\infty,
$$

where

$$
\begin{aligned}
U(t) & =S^{-1} e^{t^{2}} \int_{|t|}^{\infty} e^{-y^{2}} e^{-y^{\frac{1}{4}}} \sin y^{\frac{1}{4}} \mathrm{~d} y \\
S & =\int_{-\infty}^{\infty} e^{\xi^{2}} \int_{|\xi|}^{\infty} e^{-y^{2}} e^{-y^{\frac{1}{4}}} \sin y^{\frac{1}{4}} \mathrm{~d} y \mathrm{~d} \xi>0 .
\end{aligned}
$$

- The auto-integral representation:

$$
H_{n}(x)=\int_{-\infty}^{\infty} V(t-x) H_{n}(t) \mathrm{d} t, \quad n \geq 0,|x|<\infty,
$$

where

$$
V(t)= \begin{cases}\frac{e^{-t^{\frac{1}{4}}} \sin \left(t^{\frac{1}{4}}\right)}{\pi t}, & \text { if } t>0, \\ 0, & \text { if } t \leq 0 .\end{cases}
$$




\section{New Canonical-Integral Representation of $\left\{H_{n}\right\}_{n \geq 0}$}

First, let us recall some properties of $\left\{H_{n}\right\}_{n \geq 0},[8,10]$.

- The Taylor expansion:

$$
H_{n}(x)=\sum_{k=0}^{\left[\frac{n}{2}\right]} \frac{(-1)^{k} n !}{2^{2 k} k !(n-2 k) !} x^{n-2 k}, \quad n \geq 0 .
$$

- The symmetry property:

$$
H_{n}(-x)=(-1)^{n} H_{n}(x), \quad n \geq 0 .
$$

- The Appel property:

$$
H_{n}^{\prime}(x)=n H_{n-1}(x), \quad n \geq 0, H_{-1}(x)=0 .
$$

- The three-terms-recurrence relation:

$$
\left\{\begin{array}{l}
H_{-1}(x)=0, \quad H_{0}(x)=1, \\
H_{n+1}(x)=x H_{n}(x)-\frac{n}{2} H_{n-1}(x), \quad n \geq 0 .
\end{array}\right.
$$

Next, let $\mathscr{W}_{x}$ be the linear functional on polynomials and given by its moments

$$
H_{n}(x)=\left\langle\mathscr{W}_{x}, t^{n}\right\rangle, \quad n \geq 0,|x|<\infty .
$$

From (2.1) and (2.3), we show that

$$
\mathscr{W}_{-x}=h_{-1}\left(\mathscr{W}_{x}\right), \quad|x|<\infty .
$$

From (2.2) and (2.3), the linear functional $\mathscr{W}_{x}$ satisfies

$$
\left(\mathscr{W}_{x}\right)_{0}=1, \quad \mathscr{W}_{x}^{\prime}-2(t-x) \mathscr{W}_{x}=0, \quad|x|<\infty .
$$

Lemma 2.1. For any real number $x$, the following properties hold:

$$
\begin{aligned}
\mathscr{W}_{x} & =\tau_{x} \mathscr{W}_{0}, \\
H_{n}(x) & =\left\langle\mathscr{W}_{0},(t+x)^{n}\right\rangle, \quad n \geq 0,
\end{aligned}
$$

where $\mathscr{W}_{0}$ is symmetric (i.e., $\left.h_{-1}\left(\mathscr{W}_{0}\right)=\mathscr{W}_{0}\right)$, normalized $\left(\right.$ i.e., $\left.\left(\mathscr{W}_{0}\right)_{0}=1\right)$ and satisfying the Pearson equation $\mathscr{W}_{0}^{\prime}-2 t^{2} \mathscr{W}_{0}=0$.

Proof. Let $x$ be a fixed real number. We have $\left(\tau_{-x} \mathscr{W}_{x}\right)_{0}=\left(\mathscr{W}_{x}\right)_{0}=H_{0}(x)=1$. If we take (2.4) into account, we can write

$$
\begin{aligned}
\left\langle\left(\tau_{-x} \mathscr{W}_{x}\right)^{\prime}-2 t\left(\tau_{-x} \mathscr{W}_{x}\right), p(t)\right\rangle & =-\left\langle\mathscr{W}_{x}, p^{\prime}(t-x)\right\rangle-2\left\langle\mathscr{W}_{x},(t-x) p(t-x)\right\rangle \\
& =\left\langle\mathscr{W}_{x}^{\prime}, p(t-x)\right\rangle-2\left\langle(t-x) \mathscr{W}_{x}, p(t-x)\right\rangle \\
& =\left\langle\mathscr{W}_{x}^{\prime}-2(t-x) \mathscr{W}_{x}, p(t-x)\right\rangle \\
& =0, \quad p \in \mathbb{P} .
\end{aligned}
$$

So, the normalized linear functional $\tau_{-x} \mathscr{W}_{x}$ satisfies: $\left(\tau_{-x} \mathscr{W}_{x}\right)^{\prime}-2 t\left(\tau_{-x} \mathscr{W}_{x}\right)=0$. The fact that $\mathscr{W}_{0}$ is the unique normalized linear functional satisfying the Pearson equation $\mathscr{W}_{0}^{\prime}-2 t \mathscr{W}_{0}=0$, yields $\tau_{-x} \mathscr{W}_{x}=\mathscr{W}_{0}$ and then $\mathscr{W}_{x}=\tau_{x} \mathscr{W}_{0}$.

Finally, (2.6) follows in a straightforward way from (2.3) and (2.5). 
2.1. An integral representation of $\mathscr{W}_{x}$. At first, we start by giving an integral representation of $\mathscr{W}_{0}$ as follows

$$
\left\langle\mathscr{W}_{0}, p\right\rangle=\int_{-\infty}^{\infty} U(t) p(t) \mathrm{d} t, \quad p \in \mathbb{P}
$$

where we assume that the function $U$ is absolutely continuous on the real line and decaying as fast as its derivative $U^{\prime}$.

By an easy integration by parts, we obtain

$$
\begin{aligned}
0 & =\left\langle\mathscr{W}_{0}^{\prime}-2 t \mathscr{W}_{0}, p\right\rangle=-\left\langle\mathscr{W}_{0}, p^{\prime}(t)+2 t p(t)\right\rangle=-\int_{-\infty}^{\infty} U(t)\left(p^{\prime}(t)+2 t p(t)\right) \mathrm{d} t \\
& =-[U(t) p(t)]_{-\infty}^{\infty}+\int_{-\infty}^{\infty}\left(U^{\prime}(t)-2 t U(t)\right) p(t) \mathrm{d} t, \quad p \in \mathbb{P} .
\end{aligned}
$$

The following condition:

$$
\lim _{t \rightarrow \pm \infty} U(t) p(t)=0, \quad p \in \mathbb{P}
$$

leads to

$$
\int_{-\infty}^{\infty}\left(U^{\prime}(t)-2 t U(t)\right) p(t) \mathrm{d} t=0, \quad p \in \mathbb{P}
$$

This implies

$$
U^{\prime}(t)-2 t U(t)=\lambda f(t),
$$

where $\lambda \neq 0$ is arbitrary and the function $f$ is locally integrable, with rapid decay, and representing the null function, i.e.,

$$
\int_{-\infty}^{\infty} t^{n} f(t) \mathrm{d} t=0, \quad n \geq 0
$$

Conversely, if $U$ is a solution of (2.10) verifying the hypothesis above and the condition:

$$
\int_{-\infty}^{\infty} U(t) \mathrm{d} t \neq 0
$$

then (2.8) and (2.9) are fulfilled and (2.7) defines a linear functional $\mathscr{W}_{0}$, which is a solution of the Pearson equation $\mathscr{W}_{0}^{\prime}-2 t \mathscr{W}_{0}=0$. Putting

$$
f(t)=-\operatorname{sgn}(t) s(|t|), \quad t \in]-\infty,+\infty[,
$$

where $s$ is the Stieltjes function $[10,1,11]$,

$$
s(t)= \begin{cases}0, & t \leq 0 \\ e^{-t^{\frac{1}{4}}} \sin t^{\frac{1}{4}}, & t>0 .\end{cases}
$$


In view of the fact that $\int_{0}^{\infty} t^{n} s(t) \mathrm{d} t=0, n \geq 0$, we get

$$
\begin{aligned}
\int_{-\infty}^{\infty} t^{n} f(t) \mathrm{d} t & =-\int_{-\infty}^{\infty} t^{n} \operatorname{sgn}(t) s(|t|) \mathrm{d} t=\int_{-\infty}^{0} t^{n} s(-t) \mathrm{d} t+\int_{0}^{\infty} t^{n} s(t) \mathrm{d} t \\
& =(-1)^{n} \int_{0}^{\infty} t^{n} s(t) \mathrm{d} t+\int_{0}^{\infty} t^{n} s(t) \mathrm{d} t=\left(1+(-1)^{n}\right) \int_{0}^{\infty} t^{n} s(t) \mathrm{d} t \\
& =0, \quad n \geq 0 .
\end{aligned}
$$

Let $U$ be the function defined on the real line and given by,

$$
\left.U(t)=\lambda e^{t^{2}} \int_{|t|}^{\infty} e^{-y^{2}} s(y) \mathrm{d} y, \quad t \in\right]-\infty,+\infty[.
$$

An easy computation shows that $U^{\prime}(t)=2 t U(t)-\lambda s(t)$ for every $t \geq 0, U^{\prime}(t)=$ $2 t U(t)+\lambda s(-t)$ for every $t<0$.

Equivalently,

$$
\left.U^{\prime}(t)-2 t U(t)=\lambda f(t), \quad t \in\right]-\infty,+\infty[.
$$

For $|t|$ large, we have

$$
|U(t)| \leq|\lambda| e^{t^{2}} \int_{|t|}^{\infty} e^{-y^{2}} e^{-y^{\frac{1}{4}}} \mathrm{~d} y \leq|\lambda| e^{-\frac{1}{2}|t|^{\frac{1}{4}}} e^{t^{2}} \int_{|t|}^{\infty} e^{-y^{2}} \mathrm{~d} y \leq o\left(e^{-\frac{1}{2}|t|^{\frac{1}{4}}}\right), \quad|t| \rightarrow \infty,
$$

by the fact that,

$$
\begin{aligned}
\lim _{|t| \rightarrow \infty} e^{t^{2}} \int_{|t|}^{\infty} e^{-y^{2}} \mathrm{~d} y & =\lim _{x \rightarrow \infty} e^{x^{2}} \int_{x}^{\infty} e^{-y^{2}} \mathrm{~d} y=\lim _{x \rightarrow \infty} \frac{\int_{x}^{\infty} e^{-y^{2}} \mathrm{~d} y}{e^{-x^{2}}}=\lim _{x \rightarrow \infty} \frac{e^{-x^{2}}}{2 x e^{-x^{2}}}=\lim _{x \rightarrow \infty} \frac{1}{2 x} \\
& =0 .
\end{aligned}
$$

Hence, the condition (2.8) holds. Clearly, $\left.U \in L^{1}\right]-\infty,+\infty[$. Condition (2.11) can be written as follows:

$$
\int_{-\infty}^{\infty} U(t) \mathrm{d} t=\lambda S \neq 0
$$

where after reverse the order of integration, we get

$$
\begin{aligned}
S & =2 \int_{0}^{\infty} U(t) \mathrm{d} t=2 \int_{0}^{\infty} e^{t^{2}} \int_{t}^{\infty} e^{-y^{2}} s(y) \mathrm{d} y \mathrm{~d} t \\
& =2 \int_{0}^{\infty} e^{-y^{2}}\left(\int_{0}^{y} e^{t^{2}} \mathrm{~d} t\right) e^{-y^{\frac{1}{4}}} \sin y^{\frac{1}{4}} \mathrm{~d} y,
\end{aligned}
$$

and by making the change of the variable $x=y^{\frac{1}{4}}$, it follows that

$$
S=8 \int_{0}^{\infty} y^{3} e^{-y} F\left(y^{4}\right) \sin y \mathrm{~d} y
$$

where $F(z)=e^{-z^{2}} \int_{0}^{z} e^{t^{2}} \mathrm{~d} t, z \in \mathbb{C}$, is the Dawson function (called also the Dawson integral), [8]. The Dawson function is an entire function for all $z \in \mathbb{C}$ and remains 
bounded for all real number $z$. Recall that the Dawson function satisfies [8]

$$
\begin{aligned}
F(0) & =0, \quad F^{\prime}(z)=-2 z F(z)+1, \quad z \in \mathbb{C}, \\
F(z) & =\sum_{k=0}^{\infty} \frac{(-1)^{k} 2^{k} z^{2 k+1}}{1 \cdot 3 \cdots(2 k+1)}, \quad|z|<\infty \\
F(z) & \simeq \frac{1}{2 z}, \quad|z| \rightarrow \infty \\
F(-z) & =-F(z), \quad z \in \mathbb{C}, \\
0 \leq F(y) & \leq F_{\max }=0,541 \ldots, \quad y \geq 0
\end{aligned}
$$

where $F_{\max }=F\left(x_{\max }\right)$, with $x_{\max }=0,942 \ldots$ Notice that $x_{\max }$ is the only critical point of $F$ on the interval $[0,+\infty$. The following result contains simple but fundamental properties which will be useful in the sequel.

Lemma 2.2. The Dawson function satisfies:

$$
\begin{aligned}
& F(y)<\frac{1}{2 y} \text { if and only if } 0<y<x_{\max }, \\
& F(y)>\frac{1}{2 y} \text { if and only if } y>x_{\max }, \\
& F(y)=\frac{1}{2 y} \text { if and only if } y=x_{\max } .
\end{aligned}
$$

Proof. The proof is an immediate consequence of (2.13) and (2.14).

We can write

$$
S=\int_{0}^{\infty} G(y) \sin y \mathrm{~d} y
$$

where

$$
G(y)=8 y^{3} e^{-y} F\left(y^{4}\right), \quad y \geq 0 .
$$

From (2.16) and (2.14), we obtain

$$
0 \leq G(y) \leq 8 F_{\max } y^{3} e^{-y}, \quad y \geq 0 .
$$

Directly, $G(0)=0$ and $\lim _{y \rightarrow \infty} G(y)=0$, which implies that $G$ has a maximum for $y=\bar{y}>0$, satisfying $G^{\prime}(\bar{y})=0$, i.e.,

$$
F\left(\bar{y}^{4}\right)=\frac{4 \bar{y}^{4}}{8 \bar{y}^{8}+\bar{y}-3} .
$$

Notice that the function $G$ is decreasing on the interval $[\bar{y},+\infty[$.

Lemma 2.3. We have $\bar{y} \leq 3$.

Proof. If we suppose that $\bar{y}>3$, then $F\left(\bar{y}^{4}\right)<\frac{1}{2 \bar{y}^{4}}$. By Lemma 2.2, this yields $\bar{y}^{4}<x_{\max }=0,942 \ldots$, i.e., $\bar{y}<(0,942 \ldots)^{\frac{1}{4}}<3$. This is a contradiction.

Furthermore, the following technical lemma will be needed. 
Lemma $2.4([1])$. Consider the following integral: $S=\int_{0}^{\infty} G(x) \sin x \mathrm{~d} x$, where the function $G:[0,+\infty[\rightarrow[0,+\infty[$ is continuous on $[0,+\infty[$, decreasing on $[2 \pi,+\infty[$. Suppose that $\int_{0}^{2 \pi} G(y) \sin y \mathrm{~d} y>0$, then $S>0$.

The function $G$ given by (2.16) satisfies the condition of the previous lemma. Indeed, $G$ is a nonnegative function on $[0,+\infty$ [ and decreasing on $[2 \pi,+\infty[$. In order to show that $S$, given by $(2.15)$, is positive, it suffices to prove that $\int_{0}^{2 \pi} G(y) \sin y \mathrm{~d} y>0$. Equivalently,

$$
\int_{0}^{\pi} G(y) \sin y \mathrm{~d} y>-\int_{\pi}^{2 \pi} G(y) \sin y \mathrm{~d} y
$$

In view of Lemma 2.2 , the fact that $G \geq 0, \sin y \geq 0$, for all $y \in[0, \pi], x_{\max }^{\frac{1}{4}}<\frac{\pi}{2}$ and $\sin y \geq \frac{2}{\pi} y$ for all $y \in\left[0, \frac{\pi}{2}\right]$, we obtain

$$
\begin{aligned}
\int_{0}^{\pi} G(y) \sin y \mathrm{~d} y & \geq \int_{x_{\max }^{\frac{1}{4}}}^{\pi} y^{3} e^{-y} \sin y F\left(y^{4}\right) \mathrm{d} y \geq \int_{x_{\max }^{\frac{1}{4}}}^{\pi} \frac{y^{3} e^{-y} \sin y}{2 y^{4}} \mathrm{~d} y \\
& \geq \frac{1}{2} \int_{x_{\max }^{\frac{1}{2}}}^{\frac{\pi}{2}} e^{-y} \frac{\sin y}{y} \mathrm{~d} y \geq \frac{1}{2} \frac{2}{\pi} \int_{x_{\max }^{\frac{1}{4}}}^{\frac{\pi}{2}} e^{-y} \mathrm{~d} y \\
& \geq \frac{1}{\pi}\left(e^{-x_{\max }^{\frac{1}{4}}}-e^{-\frac{\pi}{2}}\right) .
\end{aligned}
$$

Then, we have

$$
\int_{0}^{\pi} G(y) \sin y \mathrm{~d} y \geq \frac{1}{\pi}\left(e^{-x_{\max }^{\frac{1}{4}}}-e^{-\frac{\pi}{2}}\right) \simeq 0,0263 .
$$

On the other hand, we have

$$
-\int_{\pi}^{2 \pi} G(y) \sin y \mathrm{~d} y=-\int_{\pi}^{2 \pi} y^{3} e^{-y} \sin y F\left(y^{4}\right) \mathrm{d} y \leq-F\left(\pi^{4}\right) \int_{\pi}^{2 \pi} y^{3} e^{-y} \sin y \mathrm{~d} y .
$$

By integration by parts and an easy computation we find

$$
-\int_{\pi}^{2 \pi} y^{3} e^{-y} \sin y \mathrm{~d} y=\frac{1}{2} e^{-2 \pi} \pi\left(6+12 \pi+8 \pi^{2}+e^{\pi}\left(3+3 \pi+\pi^{2}\right)\right) \simeq 1,8731
$$

and

$$
F\left(\pi^{4}\right)=e^{-\pi^{8}} \int_{0}^{\pi^{4}} e^{t^{2}} \mathrm{~d} t \leq e^{-\pi^{8}} \int_{0}^{\pi^{4}} e^{\pi^{4} t} \mathrm{~d} t=\frac{1-e^{-\pi^{8}}}{\pi^{4}} \simeq 0,010266
$$

then

$$
-\int_{\pi}^{2 \pi} G(y) \sin y \mathrm{~d} y \leq 1,8731 \cdot 0,010266 \simeq 0,01922 .
$$

From (2.17) and (2.18), we deduce that

$$
\int_{0}^{\pi} G(y) \sin y \mathrm{~d} y>-\int_{\pi}^{2 \pi} G(y) \sin y \mathrm{~d} y .
$$


Proposition 2.1. The normalized Hermite polynomial $H_{n}$ has the following integral representations:

$$
H_{n}(x)=\int_{-\infty}^{\infty} U(t-x) t^{n} \mathrm{~d} t, \quad n \geq 0,|x|<\infty
$$

where

$$
\begin{aligned}
U(t) & =S^{-1} e^{t^{2}} \int_{|t|}^{\infty} e^{-y^{2}} e^{-y^{\frac{1}{4}}} \sin y^{\frac{1}{4}} \mathrm{~d} y, \\
S & =\int_{-\infty}^{\infty} e^{\xi^{2}} \int_{|\xi|}^{\infty} e^{-y^{2}} e^{-y^{\frac{1}{4}}} \sin y^{\frac{1}{4}} \mathrm{~d} y \mathrm{~d} \xi>0 .
\end{aligned}
$$

Proof. It is a straightforward consequence of Lemma 2.1 and 2.4, and (2.12).

2.2. On the zeros of the function $U$. By the change of the variable $y=x^{4}$, the function $U$ given by (2.19), can by written as

$$
U(t)=4 S^{-1} e^{t^{2}} V\left(|t|^{\frac{1}{4}}\right), \quad|t|<\infty,
$$

where

$$
V(t)=\int_{t}^{\infty} x^{3} e^{-x^{8}-x} \sin x \mathrm{~d} x=\int_{0}^{\infty}(x+t)^{3} e^{-(x+t)^{8}-x-t} \sin (x+t) \mathrm{d} x, \quad t \geq 0 .
$$

Clearly, the function $U$ is even and their zeros are exactly those of the function $t \mapsto V\left(|t|^{\frac{1}{4}}\right)$. Observe that we have

$$
V(k \pi)=(-1)^{k} I_{k}, \quad k \geq 0
$$

where

$$
I_{k}=\int_{0}^{\infty} G_{k}(x) \sin x \mathrm{~d} x
$$

and

$$
G_{k}(x)=G_{0}(x+k \pi)=(x+k \pi)^{3} e^{-(x+k \pi)^{8}-(x+k \pi)} .
$$

Lemma 2.5. For every integer $k \geq 0$, we have $I_{k}>0$.

Proof. Let $h(x)=-8 x^{8}-x+3$ for all $x \geq 0$. So, $h^{\prime}(x)=-64 x^{7}-1<0$ for all $x \geq 0$ and $h$ is decreasing on $[0,+\infty$ [. The function $h$ is a bijection from $[0,+\infty[$ to $]-\infty, 3]$. Directly, there exists a unique solution $\theta \in[0,+\infty[$ solution of the equation: $h(x)=0$, where $x \geq 0$. By the intermediate value theorem, we can see that $\frac{1}{2}<\theta<1$, since $h\left(\frac{1}{2}\right)=\frac{9}{2}>0$ and $h(1)=-6<0$. So, $h(x)<0$, for all $\left.x \in\right] \theta,+\infty[$, and $h(x)>0$ for all $x \in\left[0, \theta\left[\right.\right.$. It is clear that $G_{0}^{\prime}(x)=x^{2} e^{-x^{8}-x} h(x)$ for all $x \geq 0$. Thus, $G_{0}$ is decreasing on $[\theta,+\infty[$. The fact that $\theta<1$ allows us to say that:

- the function $G_{0}$ is decreasing on the interval $[\pi,+\infty[$;

- the function $G_{k}$ is decreasing on the interval $[0,+\infty$ [ for every $k \geq 1$.

For every fixed integer $k \geq 1$, we have

$$
I_{k}=\lim _{n \rightarrow \infty} \int_{0}^{2 n \pi} G_{k}(x) \sin x \mathrm{~d} x
$$


Clearly,

$$
\int_{0}^{2 n \pi} G_{k}(x) \sin x \mathrm{~d} x=\sum_{l=0}^{n-1} \int_{0}^{\pi}\left(G_{k}(x+2 l \pi)-G_{k}(x+(2 l+1) \pi)\right) \sin x \mathrm{~d} x,
$$

for every integer $n \geq 1$. Since $\sin x>0$ on $] 0, \pi\left[\right.$, and all the functions $G_{k}, k \geq 1$, are decreasing on $[0,+\infty[$, we have

$$
\int_{0}^{\pi}\left(G_{k}(x+2 l \pi)-G_{k}(x+(2 l+1) \pi)\right) \sin x \mathrm{~d} x>0, \quad l \geq 0 .
$$

Accordingly, it follows that

$$
I_{k} \geq \int_{0}^{\pi}\left(G_{k}(x)-G_{k}(x+\pi)\right) \sin x \mathrm{~d} x>0, \quad k \geq 1 .
$$

For $k=0$, let's note first that $G_{0}$ is nonnegative and continuous on $[0,+\infty[$ and decreasing on $\left[2 \pi,+\infty\left[\right.\right.$. By Lemma 2.4, in order to show that $I_{0}>0$, it suffices to show that $\int_{0}^{2 \pi} G_{0}(x) \sin x \mathrm{~d} x>0$. Equivalently,

$$
\int_{0}^{\pi} G_{0}(x) \sin x \mathrm{~d} x>-\int_{\pi}^{2 \pi} G_{0}(x) \sin x \mathrm{~d} x .
$$

On the one hand, we have

$$
\int_{0}^{\pi} G_{0}(x) \sin x \mathrm{~d} x=\int_{0}^{\theta} G_{0}(x) \sin x \mathrm{~d} x+\int_{\theta}^{\pi} G_{0}(x) \sin x \mathrm{~d} x .
$$

By the fact that $G_{0}(x) \sin x \geq 0$ for every $x \in[0, \pi]$, the function $G_{0}$ is decreasing on the interval $[\theta, \pi]$, we can write

$$
\int_{\theta}^{\pi} G_{0}(x) \sin x \mathrm{~d} x \geq G_{0}(\pi) \int_{\theta}^{\pi} \sin x \mathrm{~d} x=G_{0}(\pi)(1+\cos \theta),
$$

but, $\theta \in] 0, \frac{\pi}{2}[$, then

$$
\int_{\theta}^{\pi} G_{0}(x) \sin x \mathrm{~d} x \geq G_{0}(\pi)=\pi^{3} e^{-\pi^{8}-\pi} .
$$

Since $\theta \in] \frac{\pi}{2}, \pi[\subset] 0, \pi[$, we get

$$
\int_{0}^{\theta} G_{0}(x) \sin x \mathrm{~d} x \geq e^{-\theta^{8}} \int_{0}^{\theta} x^{3} e^{-x} \sin x \mathrm{~d} x \geq e^{-1} \int_{0}^{\frac{\pi}{2}} x^{3} e^{-x} \sin x \mathrm{~d} x,
$$

by an easy computation, we obtain

$$
\int_{0}^{\frac{\pi}{2}} x^{3} e^{-x} \sin x \mathrm{~d} x=\frac{35 \sin \left(\frac{\pi}{2}\right)-19 \cos \left(\frac{\pi}{2}\right)}{16 \sqrt{e}},
$$

and hence,

$$
\int_{0}^{\theta} G_{0}(x) \sin x \mathrm{~d} x \geq \frac{35 \sin \frac{\pi}{2}-19 \cos \frac{\pi}{2}}{16 e \sqrt{e}}=\vartheta,
$$

where $\vartheta \approx 0.0014752$.

From (2.22) and (2.23), we get

$$
\int_{0}^{\pi} G_{0}(x) \sin x \mathrm{~d} x \geq \eta_{1}
$$


where $\eta_{1}=\vartheta+\pi^{3} e^{-\pi^{8}-\pi}$.

On the other hand, since $\sin x \leq 0$, for all $x \in[\pi, 2 \pi]$, we obtain

$$
-\int_{\pi}^{2 \pi} G_{0}(x) \sin x \mathrm{~d} x=-\int_{\pi}^{2 \pi} x^{3} e^{-x^{8}-x} \sin x \mathrm{~d} x \leq-e^{-\pi^{8}} \int_{\pi}^{2 \pi} x^{3} e^{-x} \sin x \mathrm{~d} x,
$$

by an easy computation, we get

$$
-\int_{\pi}^{2 \pi} x^{3} e^{-x} \sin x \mathrm{~d} x=\frac{\pi}{2} e^{-2 \pi}\left(6+12 \pi+8 \pi^{2}+e^{\pi}\left(3+3 \pi+\pi^{2}\right)\right) \approx 1.8731,
$$

and hence,

$$
-\int_{\pi}^{2 \pi} G_{0}(x) \sin x \mathrm{~d} x \leq \eta_{2},
$$

where $\eta_{2}=\beta e^{-\pi^{8}}$ and $\beta \approx 1.8731$.

Since $\eta_{1}>\eta_{2}$, the condition (2.21) holds. Thus, $I_{0}>0$.

Proposition 2.2. The function $U$, given by (2.20), has the following properties.

i) The function $U$ is even and all its zeros are placed symmetrically with respect to the origin.

ii) For every integer $k \geq 0$, $\operatorname{sgn} U\left((k \pi)^{4}\right)=(-1)^{k}$.

iii) For every integer $k \geq 0$, there exists a unique solution $\left.\xi_{k} \in\right](k \pi)^{4},((k+1) \pi)^{4}[$ solution of the equation $U(x)=0$, where $x \in\left[(k \pi)^{4},((k+1) \pi)^{4}\right]$.

Proof. The property given by i) is immediate, by taking (2.20) into account.

By $(2.20), \operatorname{sgn} U(t)=\operatorname{sgn} V\left(t^{\frac{1}{4}}\right)$ for all $t \geq 0$. Since, $V^{\prime}(x)=-t^{3} e^{-t^{8}-t} \sin (t)$ for all $t \geq 0$, then $\operatorname{sgn} V^{\prime}(t)=(-1)^{k+1}$ for all $\left.t \in\right] k \pi,(k+1) \pi[$ and all integer $k \geq 0$. We have already seen that $\operatorname{sgn} V(k \pi)=(-1)^{k}$ for all integer $k \geq 0$. Then, for every integer $k \geq 0$, there exists a unique $\left.\tau_{k} \in\right] k \pi,(k+1) \pi[$ solution to the equation $V(x)=0$, where $x \in[k \pi,(k+1) \pi]$. In view of (2.20), for every integer $k \geq 0$, we infer that $\operatorname{sgn} U\left((k \pi)^{4}\right)=(-1)^{k}$, and there exists a unique $\left.\xi_{k}=\tau_{k}^{4} \in\right](k \pi)^{4},((k+1) \pi)^{4}[$ solution of the equation $U(x)=0$, where $x \in\left[(k \pi)^{4},((k+1) \pi)^{4}\right]$. Thus, ii) and iii) hold.

\section{An Auto-Integral Representation of the Normalized Hermite POLYNOMIALS}

Recall that the Stieltjes integral formula is given by [10]

$$
\int_{0}^{\infty} x^{p-1} e^{-a x} \sin m x \mathrm{~d} x=\frac{\Gamma(p)}{\left(a^{2}+m^{2}\right)^{\frac{p}{2}}} \sin p \theta
$$

for any positive real numbers $p, q, m$, with $\sin \theta=\frac{m}{r}, \cos \theta=\frac{a}{r}, 0<\theta<\frac{\pi}{2}$ and $r=\sqrt{a^{2}+m^{2}}$. 
From (3.1) taking with $\theta=\frac{\pi}{4}$, it comes that $a=m=1$, and

$$
\int_{0}^{\infty} x^{p-1} e^{-x} \sin x \mathrm{~d} x=\frac{\Gamma(p)}{2^{\frac{p}{2}}} \sin \frac{p \pi}{4}, \quad p>0 .
$$

In particular, for $p=4(n+1)$, we get

$$
\int_{0}^{\infty} x^{4 n+3} e^{-x} \sin x \mathrm{~d} x=0, \quad n \geq 0
$$

and the transformation $x=t^{\frac{1}{4}}$, yields,

$$
\int_{0}^{\infty} t^{n} e^{-t^{\frac{1}{4}}} \sin t^{\frac{1}{4}} \mathrm{~d} t=0, \quad n \geq 0 .
$$

On the other hand, by (3.1) and the recursion property of the Gamma function, to known $\Gamma(z+1)=z \Gamma(z)$, for all $z \in \mathbb{C}$ such that $z \neq-n$ for every integer $n \geq 0$, and $\Gamma(1)=1$, we can write

$$
\int_{0}^{\infty} x^{p-1} e^{-a x} \sin m x \mathrm{~d} x=\frac{\Gamma(p+1)}{\left(a^{2}+m^{2}\right)^{\frac{p}{2}}} \frac{\sin p \theta}{p}, \quad p>0,
$$

and by letting $p \rightarrow 0^{+}$, we get

$$
\int_{0}^{\infty} \frac{e^{-a x} \sin m x}{x} \mathrm{~d} x=\theta .
$$

For $\theta=\frac{\pi}{4}, m=a=1$, the transformation $x=t^{\frac{1}{4}}$, gives us

$$
\int_{0}^{\infty} \frac{e^{-t^{\frac{1}{4}}} \sin t^{\frac{1}{4}}}{\pi t} \mathrm{~d} t=1
$$

and by taking (3.2) into account, we obtain

$$
\int_{-\infty}^{\infty} t^{n} W(t) \mathrm{d} t=\delta_{n, 0}, \quad n \geq 0
$$

where

$$
W(t)= \begin{cases}\frac{e^{-t^{\frac{1}{4}}} \sin t^{\frac{1}{4}}}{\pi t}, & \text { if } t>0 \\ 0, & \text { if } t \leq 0 .\end{cases}
$$

This leads to the following integral representation of the Dirac mass $\delta_{0}$,

$$
\left\langle\delta_{0}, p\right\rangle=\int_{-\infty}^{\infty} W(t) p(t) \mathrm{d} t=p(0), \quad p \in \mathbb{P}
$$

and more general to an integral representation of the Dirac mass $\delta_{x}$, for every real number $x$,

$$
\left\langle\delta_{x}, p\right\rangle=\int_{-\infty}^{\infty} W(t-x) p(t) \mathrm{d} t=p(x), \quad p \in \mathbb{P} .
$$

Consequently, the following auto-integral representation of the normalized $\mathcal{H}$ ermite polynomial $H_{n}$ holds

$$
H_{n}(x)=\int_{-\infty}^{\infty} W(t-x) H_{n}(t) \mathrm{d} t, \quad n \geq 0 .
$$




\section{REFERENCES}

[1] K. Ali Khelil, R. Sfaxi and A. Boukhemis, Integral representation of the generalized Bessel linear functional, Bull. Math. Anal. Appl. 9(3) (2017), 1-15.

[2] N. I. Akhiezer, The Classical Moment Problem, Oliver and Boyd, Edinburgh, London, 1965.

[3] T. S. Chihara, An Introduction to Orthogonal Polynomials, Gordon and Breach, New York, 1978.

[4] A. Ghressi and L. Kheriji, Some new results about a symmetric D-semiclassical linear form of class one, Taiwanese J. Math. 11(2) (2007), 371-382.

[5] M. E. H. Ismail and D. Stanton, Classical orthogonal polynomials as moments, Canad. J. Math. 49 (1997), 520-542.

[6] M. E. H. Ismail and D. Stanton, More orthogonal polynomials as moments, in: Mathematical Essays in Honor of Gian-Carlo Rota, Cambridge, MA, 1996, Birkhäuser Boston, Boston, MA, 1998, 377-396.

[7] M. E. H. Ismail and D. Stanton, q-Integral and moment representations for q-orthogonal polynomials, Canad. J. Math. 45 (2002), 709-735.

[8] N. N. Lebedev, Special Functions and their Applications, Translated from the Russian by Richard A. Silverman, Englewood Cliffs, New Jork, 1965.

[9] P. Maroni, Une théorie algébrique des polynômes orthogonaux, Applications aux polynômes orthogonaux semi-classiques, IMACS: International Association for Mathematics and Computers in Simulation 9 (1991), 95-130.

[10] P. Maroni, Fonctions eulériennes, polynémes orthogonaux classiques, Techniques de l'Ingénieur 154 (1994), 1-30.

[11] P. Maroni, An integral representation for the Bessel form, J. Comput. Appl. Math. 157 (1995), 251-260.

[12] M. Rahman and A. Verma, A q-integral representation for the Rogers q-ultraspherical polynomials and some applications, Constr. Approx. 2 (1986), 1-10.

[13] J. Shohat and J. D. Tamarkin, The Problem of Moments, American Mathematical Society, Providence, 1950.

[14] B. Simon, The classical moment as a selfadjoint finite difference operator, Adv. Math. 137 (1998), 82-203.

[15] G. Szegö, Orthogonal Polynomials, Fourth Edition, American Mathematical Society, Providence, 1975.

${ }^{1}$ Department of Mathematics, UNIVERSITY OF BADJI MOKHTAR, Annaba, Algeria

Email address: aboukhemis@yahoo.com

Email address: kalikhelil@gmail.com

${ }^{2}$ Coll. Edu. of Girls, Scientific Sections, University of HAFR-Al-BATIN,

Hafr-Al-Batin, Saudi Arabia

Email address: ridhasfaxi@gmail.com 

Kragujevac Journal of Mathematics

Volume 46(1) (2022), Pages 21-28.

\title{
CONNECTEDNESS OF THE CUT SYSTEM COMPLEX ON NONORIENTABLE SURFACES
}

\author{
FATEMA ALI ${ }^{1,2}$ AND FERIHE ATALAN ${ }^{1}$
}

\begin{abstract}
Let $N$ be a compact, connected, nonorientable surface of genus $g$ with $n$ boundary components. In this note, we show that the cut system complex of $N$ is connected for $g<4$ and disconnected for $g \geq 4$. We then define a related complex and show that it is connected for $g \geq 4$.
\end{abstract}

\section{INTRODUCTION}

Complexes of curves and cut system complexes of surfaces are fundamental geometric objects in geometric topology. Let $S$ be a compact, connected, orientable or nonorientable surface of genus $g \geq 1$ with $n$ boundary components. Complexes of curves, denoted by $C(S)$, have been introduced by Harvey in [3,4]. Other geometric objects on surfaces include the cut system complex introduced by Hatcher and Thurston in [5]. They have played an ever increasing role since then.

In this note, we show that the cut system complexes of nonorientable surfaces are connected for $g<4$ and disconnected for $g \geq 4$. We then introduce a related complex and show that it is connected for $g \geq 4$.

\section{Preliminaries}

Let $a$ be a simple closed curve on $S$ and let $S_{a}$ denote the surface obtained by cutting $S$ along $a$. We call $a$ on the surface $S$ nonseparating if $S_{a}$ is connected, and separating otherwise. We denote a curve or its isotopy class by the same notation throughout this article. Let $\Sigma$ be a compact, connected, orientable surface of genus $g$ with $n$ boundary components. Let us consider collections of $g$ disjoint nonseparating

Key words and phrases. A nonorientable surface, cut system complex.

2010 Mathematics Subject Classification. Primary: 57N05. Secondary: 57M99, 05C40.

DOI 10.46793/KgJMat2201.021A

Received: January 11, 2019.

Accepted: August 03, 2019. 
simple closed curves $a_{1}, a_{2}, \ldots, a_{g}$ on $\Sigma$, whose complement $\Sigma \backslash\left(a_{1} \cup \cdots \cup a_{g}\right)$ is a sphere with $2 g+n$ boundary components. The collection of their isotopy classes is called a cut system on $\Sigma$. Let $\left\langle a_{1}, a_{2}, \ldots, a_{g}\right\rangle$ be a cut system on $\Sigma$. Assume that for some $k, a_{k}^{\prime}$ is a nonseparating simple closed curve transversely intersecting $a_{k}$ at exactly one point and disjoint from all $a_{i}$ for $i \neq k$. Then if we replace $a_{k}$ by $a_{k}^{\prime}$ in the cut system, we obtain another cut system on $\Sigma$. This operation of replacing curves is called an elementary move. There are three special types of paths which is described in [5]. These special types of paths play an important role in the construction of the cut system complex. The cut system complex of a surface $\Sigma$ is a cell complex of dimension 2. Each cut system is a 0-cell (vertex) of this complex. If two cells are related by an elementary move then these two 0-cells are joined by a 1-cell (an unoriented edge) corresponding to this move. Now, we have a graph, in other words; a 1-dimensional cell complex containing the 0-cells and the 1-cells. Finally, we attach 2-cells to this graph along the boundaries resulting from the three special types of paths to get the complex.

Hatcher and Thurston showed that the cut system complex of an orientable surface is connected in [5]. Later, Wajnryb proved the same result by elementary techniques in $[6]$.

An analogous complex for nonorientable surfaces seems to be the following. Let $N$ be a compact, connected nonorientable surface of genus $g$ with $n$ boundary components. If the regular neighborhood of the curve $a$ is a Möbius band or an annulus, then we say that $a$ is one-sided or two-sided, respectively. We note that all one-sided simple closed curves on $N$ are nonseparating. In addition, there are two topological types of one-sided simple closed curves on nonorientable surfaces of odd genus $g \geq 3$. Let $a$ be a one-sided simple closed curve. We call $a$ a one-sided essential simple closed curve if either $g=1$ or $g \geq 2$ and the surface $N_{a}$ is nonorientable. Otherwise, we say that $a$ is a one-sided characteristic simple closed curve. A cut system on the nonorientable surface $N$ is defined by taking a family of pairwise disjoint one-sided essential simple closed curves. Explicitly, let $\left\{a_{1}, a_{2}, \ldots, a_{g}\right\}$ be a collection of pairwise disjoint onesided essential simple closed curves on the surface $N$. Then, the collection of their isotopy classes $\left\langle a_{1}, a_{2}, \ldots, a_{g}\right\rangle$ is said to be a cut system if the surface obtained from $N$ by cutting along all $a_{i}$ in the collection is a sphere with $g+n$ boundary components. Let $\left\langle a_{1}, a_{2}, \ldots, a_{i-1}, a_{i}, a_{i+1}, \ldots, a_{g}\right\rangle$ be a cut system on the surface $N$. Let $a_{i}^{\prime}$ be a one-sided essential simple closed curve on the surface $N$ disjoint from $a_{k}$ for $k \neq i$, $1 \leq i \leq g$ and such that it intersects $a_{i}$ at one point and does not intersect other one-sided essential simple closed curves in the collection $\left\{a_{1}, a_{2}, \ldots, a_{g}\right\}$. Similar to the orientable case, if we change $a_{i}$ by $a_{i}^{\prime}$ in the collection, and we get a new cut system $\left\langle a_{1}, a_{2}, \ldots, a_{i-1}, a_{i}^{\prime}, a_{i+1}, \ldots, a_{g}\right\rangle$. This operation, introduced by Ashiba in [1], is called an elementary move. Also, the cut system complex, denoted by $\mathcal{O}(N)$, of a nonorientable surface is described in a similar fashion to the orientable case. As we will show in the next section, unfortunately this complex is not connected for the genus $g \geq 4$. 


\section{MAIn ThEOREM}

In this section, firstly, we will explain below why $\mathcal{O}(N)$ is not connected for $g \geq 4$. Let $v_{1}=\left\langle d_{1}, a_{2}, \ldots, a_{g}\right\rangle$ and $v_{2}=\left\langle d_{2}, a_{2}, \ldots, a_{g}\right\rangle$ be vertices of the complex which are connected by an edge. Let $\bar{N}$ denote the surface $N$ whose holes are filled with discs. Since $d_{1}$ and $d_{2}$ intersect transversally once and they are disjoint from all $a_{i}$ 's we see that the homology classes of $d_{1}$ and $d_{2}$ are the same. This is because in the surface $\bar{N}$ both sums of the homology classes $\left[d_{1}\right]+\left[a_{2}\right]+\cdots+\left[a_{g}\right]$ and $\left[d_{2}\right]+\left[a_{2}\right]+\cdots+\left[a_{g}\right]$ are the Poincaré dual to the first Stiefel Whitney class so that $\left[d_{1}\right]+\left[a_{2}\right]+\cdots+\left[a_{g}\right]=\left[d_{2}\right]+\left[a_{2}\right]+\cdots+\left[a_{g}\right]$ and thus $\left[d_{1}\right]=\left[d_{2}\right]$. As a conclusion, we see that if two vertices of the complex are connected by an edge path then the homology classes of the isotopy classes in these vertices are pairwise identical in $\bar{N}$. In other words, if two vertices have a non-common homology class represented by the isotopy classes contained in them then these two vertices are not connected by an edge path. Such isotopy classes can be found for any $g \geq 4$, hence, the cut system complex cannot be connected in that case.

The above explanation raises the following question.

Question. What if we take $g-1$ pairwise disjoint one-sided essential simple closed curves $\left\{a_{1}, a_{2}, \ldots, a_{g-1}\right\}$ on $N$ as vertices, would the corresponding cut system complex made of these vertices be connected?

The answer is still negative. Indeed, if $v_{1}=\left\langle d_{1}, a_{2}, \ldots, a_{g-1}\right\rangle$ and $v_{2}=\left\langle d_{2}, a_{2}, \ldots, a_{g-1}\right\rangle$ are vertices of the complex, which are connected by an edge then cutting $N$ along all $a_{i}$ 's, we see that $d_{1}$ and $d_{2}$ are two one-sided curves inside a holed Klein Bottle intersecting transversally at one point. Hence again the homology classes $\left[d_{1}\right]$ and $\left[d_{2}\right]$ must coincide in $\bar{N}$. Therefore, the cut system complex still is not connected.

As a result of these observations, we have the following results.

Theorem 3.1. Let $N$ be a nonorientable surface of genus $g$ with $n$ holes, where $g<4$. Then the cut system complex $\mathcal{O}(N)$ is connected.

Let us take $g-2$ pairwise disjoint one-sided essential simple closed curves $\left\{a_{1}, a_{2}, \ldots, a_{g-2}\right\}$ on $N$ as vertices and let $X(N)$ denote the corresponding cut system complex made of these vertices. We call it partial cut system complex.

Theorem 3.2. Let $N$ be a nonorientable surface of genus $g$ with $n$ holes, where $g \geq 4$. Then the partial cut system complex $X(N)$ is connected.

The idea of the proofs is that any two vertices are connected by an edge path in the complex. We use Wajnryb's technique and follow his proof. The main ingredient used in the proof is the following proposition which is proved by Atalan and Korkmaz in $[2]$.

Proposition 3.1. Let $N$ be a nonorientable surface of genus $g$ with $n$ boundary components. Let $d_{1}$ and $d_{2}$ be two one-sided essential simple closed curves on the 
surface $N$ such that $i\left(d_{1}, d_{2}\right)=k$, where $k \geq 2$. In this case, there is a one-sided essential simple closed curve $d$ such that $i\left(d, d_{1}\right)<k$ and $i\left(d, d_{2}\right)<k$.

Proof of Theorem 3.1. Let $g=1$. In this case, a cut system (on the surface $N$ ) contains an isotopy class of a single curve. If two distinct one-sided curves intersect at one point, we connect them by an edge. Since any two essential one-sided curves on a genus one nonorientable surface intersect, using induction it follows from Proposition 3.1 that any two one-sided essential curves can be joined by an edge path in the cut system complex $\mathcal{O}(N)$.

Let $g=2$. Let $v_{1}$ and $v_{2}$ be any two vertices of the complex $\mathcal{O}(N)$. We will show that there exists an edge path $P=\left(v_{1}=s_{1}, s_{2}, \ldots, s_{k}=v_{2}\right)$ connecting $v_{1}$ and $v_{2}$. There are two cases.

Case 1. Suppose that the vertices $v_{1}$ and $v_{2}$ have one isotopy class of one-sided essential simple closed curve in common, say $d$. Let us cut the surface $N$ along the curve $d$. The collection of the remaining one-sided essential simple closed curves constitute two vertices of the cut system complex on the obtained surface of genus one. We have showed that the complex $\mathcal{O}(N)$ is connected for $g=1$. So, they can be connected by a path. Including this common curve $d$ to each of the vertices of this path we obtain a path in $\mathcal{O}(N)$ connecting $v_{1}$ to $v_{2}$.

Case 2. Suppose that the vertices $v_{1}$ and $v_{2}$ do not have any common isotopy class of one-sided essential simple closed curves. Let $d_{1}$ and $d_{2}$ be two different isotopy classes of one-sided essential simple closed curves on $N$ such that $v_{1}$ and $v_{2}$ contain $d_{1}$ and $d_{2}$, respectively. Then, we need to show that there exists an edge path connecting $v_{1}$ and $v_{2}$. To prove this, as in the proof of Lemma 17 in [6], we will use induction on $i\left(d_{1}, d_{2}\right)=n$.

There are three subcases.

Subcase (i). Let $i\left(d_{1}, d_{2}\right)=0$. Then there is a vertex $u$ containing both one-sided essential curves $d_{1}$ and $d_{2}$. Hence, the vertex $u$ is connected to $v_{1}$ and $v_{2}$ as in Case 1 .

Subcase (ii). Let $i\left(d_{1}, d_{2}\right)=1$. The regular neighborhood of $d_{1} \cup d_{2}$ is a two-holed real projective plane. Let us denote $N_{d_{1} \cup d_{2}}$ the surface obtained by cutting $N$ along $d_{1}$ and $d_{2}$. Since $g=2, N_{d_{1} \cup d_{2}}$ has necessarily two components, one of which is a nonorientable surface of genus one, so that we can find a one-sided essential simple closed curve disjoint from $d_{1}$ and $d_{2}$, say $e$. Now, we can find two vertices $w_{1}$ and $w_{2}$ in the complex $\mathcal{O}(N)$ which are joined by an edge and such that $w_{1}$ and $w_{2}$ contain $d_{1}$ and $d_{2}$, respectively. In other words, $w_{1}=\left\langle d_{1}, e\right\rangle$ and $w_{2}=\left\langle d_{2}, e\right\rangle$ are connected by an edge. Finally, we join $v_{1}$ to $w_{1}$ and $v_{2}$ to $w_{2}$ as in Case 1 . Therefore, we can connect $v_{1}$ and $v_{2}$.

Subcase (iii). Let $i\left(d_{1}, d_{2}\right)=n>1$. By Proposition 3.1, there is a one-sided essential simple closed curve $d$ such that $i\left(d_{1}, d\right)<n$ and $i\left(d_{2}, d\right)<n$. We choose a vertex $u$ containing $d$. By induction on $n$, we can connect the vertex $u$ to $v_{1}$ and $v_{2}$ in the cut system complex $\mathcal{O}(N)$. 
For the case $g=3$ the proof is the same as for the case $g=2$ except the Subcase (ii), which we include below.

Subcase (ii). Let $i\left(d_{1}, d_{2}\right)=1$. Since $g=3$ and $d_{1}$ and $d_{2}$ are both essential, $N_{d_{1} \cup d_{2}}$ has necessarily two components. Moreover, either both components are nonorientable of genus one or one of the components is a nonorientable surface of genus two and the other is a sphere with holes. Hence, in each case, we can find two disjoint onesided essential simple closed curves $e_{1}$ and $e_{2}$ that they are both disjoint from $d_{1}$ and $d_{2}$. Now, we can find two vertices $w_{1}$ and $w_{2}$ in the complex which are joined by an edge and such that $w_{1}$ and $w_{2}$ contain $d_{1}$ and $d_{2}$, respectively. In other words, $w_{1}=\left\langle d_{1}, e_{1}, e_{2}\right\rangle$ and $w_{2}=\left\langle d_{2}, e_{1}, e_{2}\right\rangle$ are connected by an edge. Finally, we join $w_{1}$ to $v_{1}$ and $v_{2}$ to $w_{2}$ as in Case 1 . Therefore, we can connect $v_{1}$ and $v_{2}$.

This completes the proof of the theorem.

Proof of Theorem 3.2. We use induction on the genus of the surface $N$ for $g \geq 4$.

Let $g=4$. As in the above proof, there are two cases.

Case 1. Assume that $v_{1}$ and $v_{2}$ have one isotopy class of one-sided essential curve in common, say $d$. Let $e$ and $f$ be the other one-sided essential simple closed curves of $v_{1}$ and $v_{2}$, respectively. In other words, let $v_{1}=\langle d, e\rangle$ and $v_{2}=\langle d, f\rangle$. We will show that $v_{1}$ is connected to $v_{2}$ by an edge path. In this case, there are three possibilities.

- The one-sided essential curves $e$ and $f$ are disjoint. Then, since $d$ is disjoint from both $e$ and $f$, and these all curves are one-sided essential, there is another essential simple closed curve, say $g$, in the complement of $d \cup e \cup f$. Hence, we can find an essential one-sided simple closed curve $c$ representing $\mathbb{Z}_{2}$-homology class $[e]+[f]+[g]$ such that $c$ intersects each of $e$ and $f$ at only one point. Thus, we obtain the required path.

- The one-sided essential curves $e$ and $f$ intersect at one point. Then there is nothing to prove.

- The one-sided essential curves $e$ and $f$ intersect at least two points. Let us cut the surface $N$ along the curve $d$. We get a nonorientable surface of genus three, say $N_{d}$, in which $i(e, f)=k \geq 2$. First assume that the curves $e$ and $f$ are still essential in $N_{d}$. Then, by Proposition 3.1, there is a one-sided essential simple closed curve $c$ such that $i(e, c)<k$ and $i(f, c)<k$.

Now assume without loss of generality that the curve $e$ is not essential in $N_{d}$. Hence $e$ is characteristic in $N_{d}$. Since $f$ is one-sided and $e$ is characteristic the integer $k$ must be odd. Take representatives for $e$ and $f$ and a push-off of $f$ that intersects $f$ transversally at one point. Also a take a slight perturbation $f^{\prime}$ of the push-off. By inspection we see that our curves must be as in the diagram at Figure 1.

This new curve $f^{\prime}$ has the same homology class as $f$. It has at most two components. If it is connected then it, call it $c$, intersects $f$ in one point and $e$ in $k-2$ points. Since $k-2$ is odd, $c$ is still one-sided. If the $f^{\prime}$ is not connected, then by homology arguments one of the components must be one-sided and the other one must be two-sided. The one-sided component, call it $c$ again, will intersect $f$ in at most one point and $e$ in at 


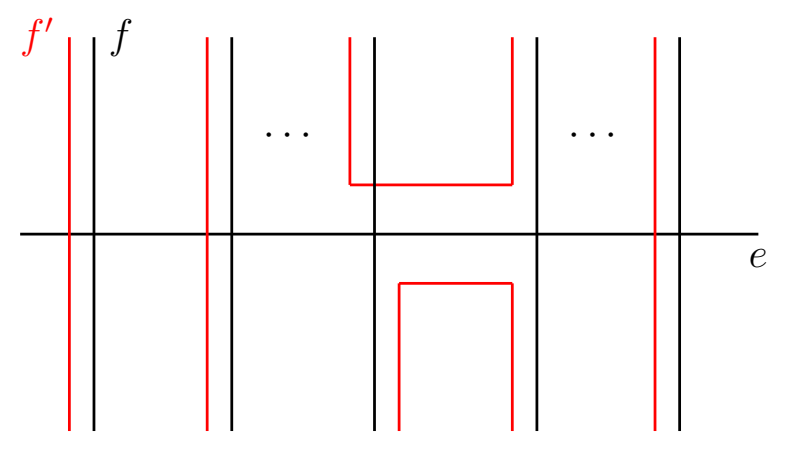

FiguRE 1.

most $k-2$ points. The curve $c$ may not be essential in $N_{d}$ but certainly is essential in $N$.

In particular, in all cases we have obtained a one-sided essential simple closed curve $c$ such that $i(e, c)<k$ and $i(f, c)<k$. Finally, using an induction argument we obtain two sequences of essential one-sided simple closed curves $e=e_{0}, e_{1}, \ldots, e_{j}=c$ and $c=c_{0}, c_{1}, \ldots, c_{s}=f$ such that any two adjacent essential one-sided simple closed curves in the sequences intersect once. Then the sequence $e=e_{0}, e_{1}, \ldots, e_{j}=c=$ $c_{0}, c_{1}, \ldots, c_{s}=f$ gives the required path in $X(N)$.

Case 2. Assume that the vertices $v_{1}$ and $v_{2}$ do not have any common isotopy class of one-sided essential simple closed curves. Let $d_{1}$ and $d_{2}$ be two different isotopy classes of one-sided essential simple closed curves on $N$ such that $v_{1}$ and $v_{2}$ contain $d_{1}$ and $d_{2}$, respectively. Then, we show that there exists an edge path connecting $v_{1}$ and $v_{2}$. To prove this, as in the proof of Lemma 17 in [6], we will use induction on $i\left(d_{1}, d_{2}\right)=n$.

There are three subcases.

Subcase (i). Let $i\left(d_{1}, d_{2}\right)=0$. Then, we can construct a vertex $u=\left\langle d_{1}, d_{2}\right\rangle$. Thus, $u$ is connected to $v_{1}$ and $v_{2}$ as in Case 1 .

Subcase (ii). Let $i\left(d_{1}, d_{2}\right)=1$. The regular neighborhood of $d_{1} \cup d_{2}$ is a two-holed real projective plane. Then, there are two possibilities. One possibility is that $N_{d_{1} \cup d_{2}}$ is a connected nonorientable surface of genus 1 (note that $N_{d_{1} \cup d_{2}}$ cannot be connected and orientable since both $d_{1}$ and $d_{2}$ are essential). Hence, we can find a one-sided essential simple closed curve disjoint from $d_{1}$ and $d_{2}$. Again we can find two vertices $w_{1}$ and $w_{2}$ containing $d_{1}$ and $d_{2}$, respectively, such that they are connected by an edge. Hence, we join $w_{1}$ to $v_{1}$ and $v_{2}$ to $w_{2}$ as in Case 1 . The other possibility is that $N_{d_{1} \cup d_{2}}$ is disconnected. We can notice that at least one component of $N_{d_{1} \cup d_{2}}$ must be nonorientable, so in this case we can also find a one-sided essential simple closed 
curve disjoint from $d_{1}$ and $d_{2}$, which allows to treat the disconnected case similarly. Therefore, we can connect $v_{1}$ and $v_{2}$ by a path.

Subcase (iii). Let $i\left(d_{1}, d_{2}\right)=n>1$. By Proposition 3.1, there is a one-sided essential simple closed curve $d$ such that $i\left(d_{1}, d\right)<n$ and $i\left(d_{2}, d\right)<n$. Let us pick a vertex $u$ containing $d$. By induction on $n$, we can join $u$ to $v_{1}$ and $v_{2}$.

Let $g \geq 5$. By the induction hypothesis, we assume that the theorem holds for a nonorientable surface of genus less than $g$. We will prove that the complex $X(N)$ is connected for a nonorientable surface of genus $g$. Let $v_{1}$ and $v_{2}$ be any two vertices of the complex $X(N)$. We will prove that these two vertices are connected by an edge path.

Case 1. Suppose that $v_{1}$ and $v_{2}$ have one isotopy class of one-sided essential curve in common, say $d$. Let us cut the surface $N$ along the curve $d$. The collection of the remaining one-sided essential simple closed curves constitute two vertices of the cut system complex on the obtained surface of smaller genus. By the induction hypothesis, they can be connected by a path. Including this common curve $d$ to each of the vertices of this path we obtain a path in $X(N)$ connecting $v_{1}$ to $v_{2}$.

Case 2. Suppose that $v_{1}$ and $v_{2}$ do not have any common isotopy classes of onesided essential simple closed curves. Let $d_{1}$ and $d_{2}$ be two different isotopy classes of one-sided essential simple closed curves on $N$ such that $v_{1}$ and $v_{2}$ contain $d_{1}$ and $d_{2}$, respectively. To prove the existence of an edge path joining $v_{1}$ and $v_{2}$, as in the proof of Lemma 17 in [6], we will use induction on $i\left(d_{1}, d_{2}\right)=n$.

There are three subcases.

Subcase (i). Let $i\left(d_{1}, d_{2}\right)=1$. The regular neighborhood of $d_{1} \cup d_{2}$ is a two-holed real projective plane. Again, we have two possibilities. One possibility is that $N_{d_{1} \cup d_{2}}$ is a connected nonorientable surface of genus $g-3$. So, one can choose pairwise disjoint $g-3$ one-sided essential curves disjoint from $d_{1}$ and $d_{2}$. Then, there are vertices $w_{1}$ and $w_{2}$ containing $d_{1}$ and $d_{2}$, respectively, such that they are joined by an edge. Thus, we connect $w_{1}$ to $v_{1}$ and $v_{2}$ to $w_{2}$ as in Case 1 . The other possibility is that $N_{d_{1} \cup d_{2}}$ is disconnected. However, by Theorem 3.10 in [2], we can find a sequence of essential one-sided simple closed curves $d_{1}=a_{1}, a_{2}, \ldots, a_{k}=d_{2}$ such that any two adjacent curves $a_{i}$ and $a_{i+1}$ in the sequence intersect once and $N_{a_{i} \cup a_{i+1}}$ is connected, where $N_{a_{i} \cup a_{i+1}}$ is the surface obtained by cutting $N$ along $a_{i}$ and $a_{i+1}$. Therefore, using the idea of the previous possibility, we can connect $v_{1}$ and $v_{2}$ by a path.

Subcase (ii). Let $i\left(d_{1}, d_{2}\right)=0$. If $\left[d_{1}\right]+\left[d_{2}\right]$ is not characteristic, then there is a vertex $u$ containing both curves $d_{1}$ and $d_{2}$. Hence, the vertex $u$ is connected to $v_{1}$ and $v_{2}$ as in Case 1 . Now, assume that $\left[d_{1}\right]+\left[d_{2}\right]$ is characteristic, in this case $g \geq 6$. Then, $\left[d_{2}\right]$ is characteristic on $N_{d_{1}}$ which is a connected nonorientable surface of genus $g \geq 5$. Without lost of generality, we can choose a one-sided essential curve $c$ on $N_{d_{1}}$, which is not characteristic such that $i\left(c, d_{2}\right)=1$. This implies that $[c]+\left[d_{1}\right]$ is not characteristic on $N$. So there is a vertex $u$ containing both curves $d_{1}$ and $c$. Now, we can connect the vertex $u$ and $v_{1}$ as in Case 1 . Moreover, $u$ can be connected to $v_{2}$ because $i\left(c, d_{2}\right)=1$ by Subcase (i) above. 
Subcase (iii). Let $i\left(d_{1}, d_{2}\right)=n>1$. By Proposition 3.1, there is a one-sided essential simple closed curve $d$ such that $i\left(d_{1}, d\right)<n$ and $i\left(d_{2}, d\right)<n$. We pick a vertex $u$ containing $d$. By induction on $n$, we can connect the vertex $u$ to $v_{1}$ and $v_{2}$ in the partial complex $X(N)$.

This finishes the proof of the theorem.

Acknowledgements. The authors would like to thank the referee for his/her valuable corrections and suggestions.

\section{REFERENCES}

[1] S. A. S. Ashiba, The Hatcher-Thurston complex on a surface, Ms. Thesis, Atilim University, Ankara, 2016.

[2] F. Atalan and M. Korkmaz, Automorphisms of curve complexes on nonorientable surfaces, Groups Geom. Dyn. 8 (2014), 39-68.

[3] W. J. Harvey, Geometric structure of surface mapping class groups, in: C. T. C. Wall (Ed.), Homological Group Theory, London Mathematical Soc. Lecture Notes Series 36, Cambridge University Press, Cambridge, 1979, 255-269.

[4] W. J. Harvey, Boundary structure of the modular group, in: I. Kra and B. Maskit (Eds.), Riemann Surfaces and Related Topics: Proceedings of the 1978 Stony Brook Conference, Annals of Math. Studies 97, Princeton University Press, Princeton, New Jersey, 1981, 245-251.

[5] A. Hatcher and W. Thurston, A presentation of the mapping class group of a closed orientable surface, Topology 19 (1980), 221-237.

[6] B. Wajnryb, An elementary approach to the mapping class group of a surface, Geometry and Topology 3 (1999), 405-466.

${ }^{1}$ Department of Mathematics, Atilim University, 06830 ANKARA, TURKey

Email address: ali.fatema@student.atilim.edu.tr

Email address: ferihe.atalan@atilim.edu.tr

${ }^{2}$ Department of Mathematics, BANI WALIED UNIVERSITY, LIBYA 
Kragujevac Journal of Mathematics

Volume 46(1) (2022), Pages 29-37.

\title{
$w M B$-PROPERTY OF ORDER $p$ IN BANACH SPACES
}

\author{
MANIJEH BAHREINI ESFAHANI ${ }^{1}$
}

\begin{abstract}
In this paper, we introduce a new property of Banach spaces called $w M B$-property of order $p(1 \leq p<\infty)$. A necessary and sufficient condition for a Banach space to have the $w M B$-property of order $p$ is given. We study $p$-convergent operators and weakly- $p$ - $L$-sets. Banach spaces with the $w M B$-property of order $p$ are characterized. Also, the Dunford-Pettis property of order $p$ and $D P^{*}$-property of order $p$ are studied in Banach spaces. Finally we show the relation between Pelczynski's property $(V)$ and $w M B$-property of order $p$.
\end{abstract}

\section{INTRODUCTION}

In 1993, J. M. F. Castillo and F. Sanchez in their fundamental paper [6], extend unconditionally converging operators and completely continuous (or Dunford-Pettis) operators to the general case by introducing $p$-convergent operators, $1 \leq p \leq \infty$. First the authors introduced weakly- $p$-summable sequences and weakly-p-convergent sequences, in the case of $p=\infty$, weakly- $\infty$-convergent sequences are simply the weaky convergent sequences. Then they introduced $p$-convergent operators. The 1 -convergent operators are simply the unconditionally converging operators and $\infty$ convergent operators are simply the completely continuous operators.

Ghenciu in 2018, introduced the concept of weakly- $p$ - $L$-set in a dual space $1 \leq p<$ $\infty$. A weakly- $q$ - $L$-set in $X^{*}$ is a weakly- $p$ - $L$-set in $X^{*}$ if $p<q[14]$.

In this paper, $p$-convergent operators and weakly- $p$ - $L$-set in $X^{*}$ are used to introduce a new property of Banach spaces called $w M B$-property of order $p, 1 \leq p<\infty$. We prove that a Banach space $X$ has $w M B$-property of order $p$ provided every unconditionally converging operator $T: X \rightarrow \ell_{\infty}$ is $p$-convergent.

Key words and phrases. $p$-Convergent operators, weakly- $p$ - $L$-sets, Dunford-Pettis property of order $p$.

2010 Mathematics Subject Classification. Primary: 46B20. Secondary: 46B25, 46B28.

DOI 10.46793/KgJMat2201.029E

Received: July 07, 2019.

Accepted: August 16, 2019. 
Carrion, Galindo and Lourenco in [5] define and discuss the variant of the classical Dunford-Pettis property, called $\left(D P^{*} P\right)$ in Banach spaces. In [18], E. D. Zeekoei and J. H. Fourie introduced the $D P^{*}$-property of order $p$ on Banach spaces $\left(D P^{*} P_{p}\right)$. Clearly, in case of $p=\infty$, we have $D P^{*} P=\left(D P^{*} P_{\infty}\right)$.

In [6], Castillo and Sanchez also introduced the Dunford-Pettis property of order $p$ in Banach spaces in $1 \leq p<\infty$, which is a generalization of the classical Dunford-Pettis property.

Finally, the concepts of the Dunford-Pettis property of order $p$ and $D P^{*}$-property of order $p$ are studied in Banach spaces. We prove that $(w M B)$ property of order $p$ implies the Dunford-Pettis property of order $p$ in Banach spaces.

1.1. Definitions and Notation. By an operator, we mean a bounded linear operator. Let $X, Y$ be Banach spaces. We denote by $L(X, Y)$ the space of all operators from $X$ into $Y$. For an operator $T: X \rightarrow Y$, the adjoint of $T$ is denoted by $T^{*}$. For a Banach space $X$, the closed unit ball of $X$ is denoted by $B_{X}$ and the identity map on $X$ is denoted by $I_{X}$. We denote by $K(X, Y)$ the space of all compact operators from $X$ into $Y$ and $W(X, Y)$ the space of all weakly compact operators from $X$ into $Y$.

Our discussion will make use of several definitions from the paper [6]. Let $X$ be a Banach space.

First we recall the definition of the weakly-p-summable sequences.

Let $1 \leq p<\infty, p^{*}$ denotes the conjugate of $p$. If $p=1, \ell_{p^{*}}$ plays the role of $c_{0}$. The unit vector basis of $\ell_{p}$ is denoted by $\left(e_{n}\right)$.

Let $1 \leq p<\infty$ and $X$ be a Banach space. The set of all $p$-summable sequences in $X$ with the natural norm

$$
\left\|\left(x_{n}\right)\right\|_{p}=\left(\sum_{i=1}^{\infty}\left|x_{n}\right|^{p}\right)^{\frac{1}{p}}
$$

is denoted by $\ell_{p}(X)$.

Let $1 \leq p<\infty$. A sequence $\left(x_{n}\right)$ in a Banach space $X$ is called weakly $p$-summable if $\left(x^{*} x_{n}\right) \in \ell_{p}$, for all $x^{*} \in X^{*}$. In other words, a sequence $\left(x_{n}\right)$ in $X$ is weakly $p$-summable if

$$
\sum_{n=1}^{\infty}\left|\left\langle x_{n}, x^{*}\right\rangle\right|^{p}<\infty
$$

for each $x^{*} \in X^{*}$ (see [9]). The set of all weakly $p$-summable sequences in $X$, endowed with the norm

$$
\left\|\left(x_{n}\right)_{n}\right\|_{p}{ }^{w}=\sup \left\{\left(\sum_{i=1}^{\infty}\left|\left\langle x_{i}, x^{*}\right\rangle\right|^{p}\right)^{\frac{1}{p}}: x^{*} \in B_{X^{*}}\right\}
$$

is denoted by $\ell_{p}^{w}(X)$.

For $p=1$, weakly 1-summable sequences correspond to weakly unconditionally converging series; For $p=\infty$, weakly $\infty$-summable sequences are just weakly null sequences in $X$, i.e., sequences which are in $c_{0}{ }^{w}(X)$. 
Ansari in 1995 showed that for $1<p<\infty$, we have $L\left(\ell_{p^{*}}, X\right)=K\left(\ell_{p^{*}}, X\right)$ if and only if weakly-p-summable sequences in $X$ are norm null, i.e., if and only if $i d_{X} \in C_{p}(X, X)$ (see [1]).

Let $1 \leq p \leq \infty$. A sequence $\left(x_{n}\right)$ in a Banach space $X$ is said to be weakly- $p$ convergent to $x \in X$ if the sequence $\left(x_{n}-x\right)$ is weakly- $p$-summable in $X$ (see [6]). The weakly- $\infty$-convergent sequences are simply the weakly convergent sequences.

Recall that a series $\sum x_{n}$ is weakly unconditionally converging if and only if $\sum\left|x^{*}\left(x_{n}\right)\right|<\infty$ for each $x^{*} \in X^{*}$ and the series $\sum x_{n}$ is unconditionally converging if and only if every rearrangement converges in the norm topology of $X$.

An operator $T: X \rightarrow Y$ is unconditionally converging if it maps weakly 1-summable sequences to (unconditionally) 1-summable sequences, i.e., $T$ takes weakly unconditionally converging series in $X$ into unconditionally convergent series in $Y$. The set of all unconditionally converging operators from $X$ to $Y$ will be denoted by $U C(X, Y)$.

An operator $T: X \rightarrow Y$ is completely continuous (Dunford-Pettis) if it maps weakly null sequences to norm null sequences. The set of all completely continuous operators from $X$ to $Y$ will be denoted by $C C(X, Y)$.

In [3], space of unconditionally converging operators and space of completely continuous operators have been studied. Recently in [2], the author investigated how some localized properties can be used to study more global structure properties.

Finally, let us recall the definition of the $L$ - sets and $V$-sets in dual spaces.

A bounded subset $A$ of $X^{*}$ is called a $V$-subset of $X^{*}$ if

$$
\lim _{n}\left(\sup \left\{\left|x^{*}\left(x_{n}\right)\right|: x^{*} \in A\right\}\right)=0,
$$

for each weakly unconditionally converging series $\sum x_{n}$ in $X$.

A Bounded subset $A$ of $X^{*}$ is called an $L$-subset of $X^{*}$ if

$$
\lim _{n}\left(\sup \left\{\left|x^{*}\left(x_{n}\right)\right|: x^{*} \in A\right\}\right)=0,
$$

for each weakly null sequence $\left(x_{n}\right)$ in $X$.

The reader is referred to Diestel [8] or Dunford-Schwartz [10] for undefined notation and terminology.

\section{MAin Results}

We begin this section with a simple, but extremely useful, characterization of $p$-convergent operators. The concept of $p$-convergent operators for $1 \leq p \leq \infty$ is introduced in [6]. It is well-known that $p$-summing operators take weakly $p$-summable sequences to $p$-summable sequences.

Let $1 \leq p \leq \infty$. An operator $T: X \rightarrow Y$ is called $p$-convergent if $T$ transforms weakly-p-summable sequences into norm-null sequences. The class of $p$-convergent operators from $X$ into $Y$ is denoted by $C_{p}(X, Y)$.

The 1-convergent operators are precisely the unconditionally converging operators and $\infty$-convergent operators are precisely the completely continuous operators. Obviously, $C_{q}(X, Y) \subset C_{p}(X, Y)$, when $p<q$. 
In this section, we study weakly- $p$ - $L$ - sets and $1 \leq p<\infty$ and $V$-sets, and then introduce a new property for Banach spaces.

Ghenciu in [14], introduced the concept of weakly- $p$ - $L$-set, $1 \leq p<\infty$, in dual space.

Let $1 \leq p<\infty$. A Bounded set $A$ in $X^{*}$ is called a weakly- $p$ - $L$-set in $X^{*}$ if

$$
\lim _{n}\left(\sup \left\{\left|x^{*}\left(x_{n}\right)\right|: x^{*} \in A\right\}\right)=0,
$$

for each weakly $p$-summable sequences $\left(x_{n}\right)$ in $X$.

The weakly-1- $L$-set in $X^{*}$ are precisely the $V$-subset of $X^{*}$. If $p<q$, then a weakly $p$-summable sequences in $X$ is a weakly $q$-summable sequences in $X$, i.e., $\ell_{p}{ }^{w}(X) \subseteq \ell_{q}{ }^{w}(X)$. Hence a weakly- $q$ - $L$-set in $X^{*}$ is a weakly- $p$ - $L$-set in $X^{*}$, and thus every weakly- $q$ - $L$-set in $X^{*}$ is a $V$-set of $X^{*}$ for $1<q$. In the following, we will give equivalent characterizations of Banach spaces which the converse statement holds.

Now we are ready to give our new property for Banach spaces using the concept of weakly- $p$ - $L$-sets.

Definition 2.1. Let $1 \leq p<\infty$. A Banach space $X$ has the $w M B$-property of order $p\left(w M B_{p}\right)$ if every $V$-set in $X^{*}$ is a weakly- $p$ - $L$ - set of $X^{*}$.

Recall that $T: Y \rightarrow X$ is an unconditionally converging operator if and only if $T^{*}\left(B_{Y^{*}}\right)$ is a $V$-set in $X^{*}$. Ghenciu generalized this characterization of unconditionally converging operators to $p$-convergent operators in terms of weakly- $p$ - $L$-sets and compact operators [14]. The following two theorems, which give a characterization of $p$-convergent operators, play an important role in this study.

Theorem 2.1 ([14], Theorem 13). Let $1 \leq p<\infty$. Let $T: Y \rightarrow X$ be an operator. The following are equivalent:

(i) $T$ is p-convergent;

(ii) for any operator $S: \ell_{p^{*}} \rightarrow X$ if $1<p<1$ (resp. $S: c_{0} \rightarrow X$ if $p=1$ ), the operator $T S$ is compact.

Theorem 2.2 ([14], Theorem 14). Let $1 \leq p<\infty$. Let $T: Y \rightarrow X$ be an operator. The following are equivalent:

(i) $T^{*}\left(B_{X^{*}}\right)$ is a weakly-p-L-set;

(ii) $T$ is p-convergent.

In the next theorem which is our main result, we characterize Banach spaces with $w M B$-property of order $p$. A necessary and sufficient condition for a Banach space to have the $w M B$-property of order $p$ has given. We prove that a Banach space $X$ has $w M B$-property of order $p$ if and only if for every Banach space $Y$, every unconditionally converging operator $T: X \rightarrow Y$ is $p$-convergent.

Theorem 2.3. Let $1 \leq p<\infty$. The following statements are equivalent about a Banach space $X$.

(i) X has the $w M B$-property of order $p$. 
(ii) For every Banach space $Y$, if $T: X \rightarrow Y$ is an unconditionally converging operator, then $T$ is p-convergent.

(iii) Same as (ii) with $Y=\ell_{\infty}$.

Proof. $(i) \Rightarrow($ ii $)$ Suppose that $Y$ is a Banach space and $T: X \rightarrow Y$ is unconditionally converging. Then $T^{*}\left(B_{Y^{*}}\right)$ is a $V$-set, and hence is weakly- $p$ - $L$-set in $X^{*}$, since $X$ has the $w M B$-property of order $p$. Note that, for all weakly $p$-summable sequences $\left(x_{n}\right)$ in $X$

$$
\left\|T\left(x_{n}\right)\right\|=\sup \left\{\left|\left\langle x_{n}, T^{*}\left(y^{*}\right)\right\rangle\right|: y^{*} \in B_{Y}^{*}\right\} .
$$

Thus $T$ is $p$-convergent.

(ii) $\Rightarrow($ iii $)$ is obvious.

$($ iii $) \Rightarrow(i)$ Suppose that $A$ is a $V$-set in $X^{*}$ and $\left(x_{n}^{*}\right)$ be a sequence in $A$. Define $T: \ell_{1} \rightarrow X^{*}$ by $T(b)=\sum_{n} b_{n} x_{n}^{*}$ for $b=\left(b_{n}\right) \in \ell_{1}$. Note that $T_{\left.\right|_{X}}^{*}: X \rightarrow \ell_{\infty}$ and $T^{*}(x)=\left(x_{i}^{*}(x)\right)$. Let $\left(x_{n}\right)$ be a weakly $p$-summable sequence in $X$, then $\left(x_{n}\right)$ is a weakly 1 -summable sequence, since $p>1$. But $A$ is a $V$-set, hence

$$
\left\|T^{*}\left(x_{n}\right)\right\|=\sup _{i}\left|x_{i}^{*}\left(x_{n}\right)\right| \longrightarrow 0 .
$$

Therefore, $T_{\left.\right|_{X} ^{*}}^{*}$ is unconditionally converging, and hence $T_{\left.\right|_{X}}^{*}$ is $p$-convergent. Let $\left(x_{n}\right)$ be a weakly $p$-summable in $X$ and $y \in B_{\ell_{1}}$. Then

$$
\left|T(y)\left(x_{n}\right)\right|=\left|T^{*}\left(x_{n}\right)(y)\right| \leq\left\|T^{*}\left(x_{n}\right)\right\| \longrightarrow 0 .
$$

Thus, $T\left(B_{\ell_{1}}\right)$ is a weakly- $p$ - $L$-set in $X^{*}$, and hence $X$ has $w M B$-property of order $p$.

Our first result gives a characterization of unconditionally converging operators in terms of weakly- $p$ - $L$-sets.

Corollary 2.1. Let $1 \leq p<\infty$. The following statements are equivalent about a Banach space $X$.

(i) $X$ has the $w M B$-property of order $p$.

(ii) If $T: X \rightarrow Y$ is an unconditionally converging operator, for every Banach space $Y$, then $T^{*}$ maps bounded sets in $Y^{*}$ onto weakly-p-L-sets in $X^{*}$.

Another result from Theorem 2.3 gives a characterization of Banach spaces with the $w M B$-property of order $p$ in terms of weakly $p$-convergent operators. Let us recall definition of the weakly $p$-convergent operators from [18].

Let $1 \leq p \leq \infty$. An operator $T: X \rightarrow Y$ is called weak $p$-convergent if $\left(y_{n}^{*}\left(T x_{n}\right)\right)$ converges to 0 for every sequence $\left(x_{n}\right) \in \ell_{p}{ }^{w}(X)$ and every weakly null sequence $\left(y_{n}^{*}\right)$ in $Y^{*}$.

Obviously, each $p$-convergent operator is weak $p$-convergent. Zeekoei and Fourie showed that, for $1 \leq p<\infty$ and operator $T: Y \rightarrow X, T$ is weak $p$-convergent if and only if for every weakly compact operator $S: Y \rightarrow Z$, the operator $S T$ is $p$-convergent. If $S$ be the identity map on $Y$, then we have the following result. 
Corollary 2.2. Let $1 \leq p<\infty$. Let $X$ and $Y$ be Banach spaces and $Y$ be reflexive. Then $X$ has the $w M B$-property of order $p$ if and only if every unconditionally converging operator $T: X \rightarrow Y$ is weak p-convergent.

In Theorem 2.3, we showed that if $T: X \rightarrow Y$ is $p$-convergent whenever $T$ is an unconditionally converging, then $X$ has the $w M B$-property of order $p$. In the next theorem we extend this result to the second adjoint operators. In fact, we show that $X$ has the $w M B$-property of order $p$ whenever unconditionally converging operator $T$ implies that the second adjoint operator $T^{* *}$ is $p$-convergent.

Theorem 2.4. Let $1 \leq p<\infty$. Let $X$ be a Banach space and $T: X \rightarrow Y$ is an operator, for every Banach space $Y$. If $T^{* *}$ is p-convergent whenever $T$ is unconditionally converging, then $X$ has the $w M B$-property of order $p$.

Proof. Suppose $T: X \rightarrow Y$ is an unconditionally converging operator. Then by assumption, the second adjoint operator $T^{* *}: X^{* *} \rightarrow Y^{* *}$ is $p$-convergent. Now, let $\eta_{X}: X \rightarrow X^{* *}$ and $\eta_{Y}: Y \rightarrow Y^{* *}$ be the natural embedding of $X$ onto $X^{* *}$ and $Y^{* *}$, respectively. Thus, $T^{* *} \eta_{X}$ is $p$-convergent, and hence $\eta_{Y} T$ is $p$-convergent. Let $S: \ell_{p^{*}} \rightarrow X$ if $1<p<1$ (resp. $S: c_{0} \rightarrow X$ if $p=1$ ), hence operator $\eta_{Y} T S$ is compact by Theorem 2.1, thus $T S$ is compact and again using Theorem 2.1, $T$ is $p$-convergent. Finally, Theorem 2.3, implies that $X$ has the $w M B$-property of order $p$.

Now we study the relation between the $w M B$-property of order $p$ and the DunfordPettis property of order $p$.

A Banach space $X$ has the Dunford-Pettis property if for every Banach space $Y$, every weakly compact operator $T: X \rightarrow Y$ transforms weakly compact sets of $X$ into norm compact sets in $Y$, i.e., $W(X, Y) \subseteq C C(X, Y)$. In other words, for every Banach space $Y$, every weakly compact operator $T: X \rightarrow Y$ is completely continuous.

Castillo and Sanchez introduced a weaker property called Dunford-Pettis property of order $p\left(D P P_{p}\right)$ (see [6]).

Let $1 \leq p \leq \infty$. A Banach space $X$ has the Dunford-Pettis property of order $p$ if every weakly compact operator $T: X \rightarrow Y$ is $p$-convergent, for any Banach space $Y$, i.e., $W(X, Y) \subseteq C_{p}(X, Y)$.

Clearly Dunford-Pettis property of order $p$ implies Dunford-Pettis property of order $q$ whenever $p<q$. Also Dunford-Pettis property of order $\infty$ is precisely Dunford-Pettis property and every Banach space has Dunford-Pettis property of order 1.

Next theorem shows that the $w M B$-property of order $p$ implies the Dunford-Pettis property of order $p$.

Theorem 2.5. Let $1 \leq p<\infty$. Let Banach space $X$ has the $w M B$-property of order $p$. Then $X$ has the Dunford-Pettis property of order $p$.

Proof. Let $Y$ be any Banach space and $T: X \rightarrow Y$ is weakly compact. Then it is easy to see that $T$ is unconditionally converging operator. By using Theorem 2.3, $T$ is $p$-convergent, since $X$ has $w M B$-property of order $p$. 
Finally, the concepts of the Dunford-Pettis property of order $p$ and $D P^{*}$-property of order $p$ are studied in Banach spaces.

Carrion, Galindo and Lourenco in [5] define and discuss the variant of the classical Dunford-Pettis property, called $\left(D P^{*} P\right)$ in Banach spaces.

A Banach space $X$ has the $D P^{*}$-property $\left(D P^{*} P\right)$ if every weakly compact sets in $X$ are limited. In other words, every $w^{*}$-null sequence $\left(x_{n}^{*}\right)$ in $X^{*}$, converges uniformly to 0 on all weakly compact sets in $X$.

Zeekoei and Fourie in [18], introduced the $D P^{*}$-property of order $p$ on Banach spaces $\left(D P^{*} P_{p}\right)$.

Let $1 \leq p \leq \infty$. A Banach space $X$ has the $D P^{*}$-property of order $p\left(D P^{*} P_{p}\right)$ if every weakly- $p$-compact sets in $X$ are limited.

Clearly, $D P^{*} P_{q}$ implies $D P^{*} P_{p}$ if $p<q$. Also the $D P^{*}$-property implies $D P^{*}$ property of order $p$ for $1 \leq p<\infty$ and $D P^{*} P=\left(D P^{*} P_{\infty}\right)$.

We prove that $w M B$-property of order $p$ implies the Dunford-Pettis property of order $p$ in Banach spaces.

Theorem 2.6. Let $1 \leq p<\infty$. Let $X$ be a Grothendick space with the $w M B$-property of order $p$. Then $X$ has the $D P^{*}$-property of order $p$.

Proof. Since Banach space $X$ is Grothendick, the Dunford-Pettis property of order $p$ implies the $D P^{*}$-property of order $p$. Now, Theorem 2.5 gives the result.

Corollary 2.3. Let $1 \leq p<\infty$. Let $X$ be a reflexive space with the $w M B$-property of order $p$. Then $X$ has the $D P^{*}$-property of order $p$.

The following result gives another characterization of Banach spaces witch have the $w M B$-property of order $p$.

Corollary 2.4. Let $1 \leq p<\infty$. Let Banach space $X$ has the $w M B$-property of order $p$. Then every weakly compact operator $T$ from $X$ into $c_{0}$ is p-convergent.

It was shown in [14] that if $1 \leq p<\infty$ and $A$ is a bounded subset of a Banach space $X$, then $A$ is weakly- $p$ - $L$-subset of $X^{*}$ if and only if $\left(x_{n}\right)$ is a weakly $p$-summable sequence in $X$ and $\left(x_{n}^{*}\right)$ is a sequence in $X^{*}$, then $\lim x_{n}^{*}\left(x_{n}\right)=0$. We generalize this result to weakly $p$-summable sequences in $X$ and weakly null sequences $\left(x_{n}^{*}\right)$ in $X^{*}$ if $X$ has the $w M B$-property of order $p$.

Theorem 2.7. Let $1 \leq p<\infty$. Let Banach space $X$ has the $w M B$-property of order $p$. If $\left(x_{n}\right)$ is a weakly $p$-summable sequence in $X$ and $\left(x_{n}^{*}\right)$ is a weakly null sequence in $X^{*}$, then $\lim x_{n}^{*}\left(x_{n}\right)=0$.

Proof. Suppose $\left(x_{n}\right)$ is a weakly $p$-summable sequence in $X$ and $\left(x_{n}^{*}\right)$ is a weakly null sequence in $X^{*}$. Let $T: X \rightarrow c_{0}$ such that $T x=\left(x_{n}^{*}\right)$. Thus, $T^{*}$ is weakly compact and $T$ is $p$-convergent by Corollary 2.4 Therefore, $\left(T x_{n}\right)$ is norm-null sequence, and hence $\lim x_{n}^{*}\left(x_{n}\right)=0$. 
In our last result we investigate the converse of Theorem 2.5. We show that if a Banach space $X$ has the $w M B$-property of order $p$, then $X$ has the Dunford-Pettis property of order $p$ for $1 \leq p<\infty$.

Recall that a Banach space $X$ has Pelczynski's property $(V)$ if every $V$-subset of $X^{*}$ is weakly sequentially compact in the weak topology of $X^{*}$. Equivalently, $X$ has Pelczynski's property $(V)$ if for every Banach space $Y$, every unconditionally converging operator $T: X \rightarrow Y$ is weakly compact (see [15]).

Theorem 2.8. Let $1 \leq p<\infty$. Let $X$ be a Banach space with Dunford-Pettis property of order $p$. If $X$ has Pelczynski's property $(V)$, then $X$ has wMB-property of order $p$.

Proof. Suppose $Y$ is any Banach space and operator $T: X \rightarrow Y$ is unconditionally converging. Then $T$ is weakly compact, since $X$ has the Pelczynski's property $(V)$. Now as $X$ has the Dunford-Pettis property of order $p$, then $T$ is $p$-convergent. Thus, $X$ has $w M B$-property of order $p$.

\section{REFERENCES}

[1] S. I. Ansari, On Banach spaces $Y$ for which $B(C(\Omega, Y)=K C(\Omega, Y)$, Pacific J. Math. 169(2) (1995), 201-218.

[2] M. E. Bahreini, Dunford-Pettis sets, $V^{*}$-sets, and property $\left(M B^{*}\right)$, Iran. J. Sci. Technol. Trans. A Sci. 42(4) (2018), 2289-2292.

[3] M. E. Bahreini, E. M. Bator and I. Ghenciu, Complemented subspaces of linear bounded operators, Canad. Math. Bull. 55(3) (2012), 449-461.

[4] E. M. Bator, Remarks on completely continuous operators, Bull. Pol. Acad. Sci. Math. 37 (1987), 409-413.

[5] H. Carrion, P. Galindo and M. L. Lourenco, A stronger Dunford-Pettis, Studia Math. 3 (2008), 205-216.

[6] J. Castillo and F. Sanchez, Dunford-Pettis like properties of countinuous vector function spaces, Revista Mathematica de la Universidad Complutense de Madrid 6 (1993), 43-59.

[7] J. Diestel, A survey of results related to the Dunford-Pettis property, Contemp. Math. 2 (1980), $15-60$.

[8] J. Diestel, Sequences and Series in Banach Spaces, Grad Texts in Math 92, Springer-Verlag, Berlin, 1984.

[9] J. Diestel, H. Jarchow and A. Tonge, Absolutely Summing Operators, Cambridge Stud. Adv. Math. 43, Cambridge University Press, Cambridge, 1995.

[10] N. Dunford and J. T. Schwartz, Linear Operators, Part I: General Theory, Wiley-Interscience, New York, 1958.

[11] G. Emmanuele, Banach spaces in which Dunfrd-Pettis sets are relatively compact, Arch. Math. 58 (1992), 477-485.

[12] H. Fourie and J. Swart, Banach ideals of p-convergent operators, Manuscripta Math. 26 (1979), 349-362.

[13] H. Fourie and D. Zeekoei, DP*-properties of order $p$ on Banach spaces, Quaest. Math. 37(3) (2014), 349-358.

[14] I. Ghenciu, The p-Gelfand Phillips property in spaces of operators and Dunford-Pettis like sets, Acta Math. Hungar. 155 (2018), 439-457.

[15] A. Pelczynski, Banach spaces on which every unconditionally converging operator is weakly compact, Bull. Acad. Polon. Sci., Sér. Sci. Math. Astronom. Phys. 10 (1962), 641-648. 
[16] P. Saab and B. Smith, Spaces on which every unconditionally converging operators are weakly completely continuous, Rocky Mountain J. Math. 22(3) (1992), 1001-1009.

[17] T. Schlumprecht, Limited sets in Banach spaces, Dissertation, Munich, 1987.

[18] E. D. Zeekoei and J. H. Fourie, On p-convergent operators on Banach Lattices, Acta Math. Sin. 34(5) (2018), 873-890.

${ }^{1}$ Department of Mathematics,

UNIVERSITY OF KHANSAR,

KHANSAR, IRAN

Email address: mebahreini@yahoo.com

Email address: mebahreini@khansar-cmc.ac.ir 

Kragujevac Journal of Mathematics

Volume 46(1) (2022), Pages 39-47.

\title{
REFINING SOME INEQUALITIES FOR FRAMES WITH SPECHT'S RATIO
}

\section{FAHIMEH SULTANZADEH ${ }^{1}$, MAHMOUD HASSANI ${ }^{1}$, MOHSEN ERFANIAN OMIDVAR ${ }^{1}$, AND RAJAB ALI KAMYABI GOL ${ }^{2}$}

\begin{abstract}
We give a new lower bound in some inequalities for frames in a Hilbert space. If $\left\{f_{i}\right\}_{i \in I}$ is a Parseval frame for the Hilbert space $\mathbb{H}$ with frame operator $S f=\sum_{i \in I}\left\langle f, f_{i}\right\rangle f_{i}$, then, for every $J \subset I$ and $f \in \mathbb{H}$, we have

$$
\left(\frac{1+2 \alpha}{2+2 \alpha}\right)\|f\|^{2} \leq \sum_{i \in J}\left|\left\langle f, f_{i}\right\rangle\right|^{2}+\left\|\sum_{i \in J^{c}}\left\langle f, f_{i}\right\rangle f_{i}\right\|^{2},
$$

where $\alpha=\inf \left\{R\left(\frac{\left\|S_{J^{c}} f\right\|}{\left\|S_{J} f\right\|}\right): f \in \mathbb{H}, J \subset I\right\}$ with Specht's ratio $R$. Also we obtain some improvements of the inequalities for general frames and alternate dual frames under suitable conditions. Our results refine the remarkable results obtained by Balan et al. and Gavruta.
\end{abstract}

\section{INTRODUCTION}

Frame theory was introduced by Duffin and Schaeffer [6] in 1952. Frames are an essential tool for many emerging applications. Their main advantage is the fact that frames can be designed to be redundant while still providing reconstruction formulas. Due to their numerical stability, tight frames and, in particular, Parseval frames are of increasing interest in applications (see $[5,7,13])$. Let $(\mathbb{H},\langle\cdot, \cdot\rangle)$ be a separable Hilbert space. We denote by $L(\mathbb{H})$ the algebra of all linear operators on $\mathbb{H}$. The space $\ell^{2}(I)$ is the set of $\left\{a_{i}\right\}_{i \in I}$ such that $a_{i} \in \mathbb{C}$ and $\sum_{i \in I}\left|a_{i}\right|^{2}<\infty$ when $I$ is a finite or countable set. A frame for $\mathbb{H}$ is a family of vectors $F=\left\{f_{i}\right\}_{i \in I}$ in $\mathbb{H}$ which satisfies

$$
A\|f\|^{2} \leq \sum_{i \in I}\left|\left\langle f, f_{i}\right\rangle\right|^{2} \leq B\|f\|^{2}, \quad \text { for every } f \in \mathbb{H},
$$

Key words and phrases. Specht's ratio, frame, Parseval frame, inequality. 2010 Mathematics Subject Classification. Primary: 42C15.

DOI 10.46793/KgJMat2201.039S

Received: May 18, 2019.

Accepted: August 16, 2019. 
for positive constants $0<A \leq B$. The bounded, invertible, and positive linear operator $S: \mathbb{H} \rightarrow \mathbb{H}$ defined by

$$
S f=\sum_{i \in I}\left\langle f, f_{i}\right\rangle f_{i}, \quad f \in \mathbb{H}
$$

is known as the frame operator associated to $F$. It allows reconstruction of each vector $f \in \mathbb{H}$ in terms of the family $F$ as follows:

$$
f=\sum_{i \in I}\left\langle f, S^{-1} f_{i}\right\rangle f_{i}=\sum_{i \in I}\left\langle f, f_{i}\right\rangle S^{-1} f_{i} .
$$

If $F$ is a Parseval frame, that is, $S=i d$, then the reconstruction formula resembles the Fourier series of $f$ associated to an orthonormal basis $B=\left\{b_{j}\right\}_{j \in J}$ of $\mathbb{H}$ :

$$
f=\sum_{j \in J}\left\langle f, b_{j}\right\rangle b_{j}
$$

but the frame coefficients $\left\{\left\langle f, f_{i}\right\rangle\right\}_{i \in I}$ given by $F=\left\{f_{i}\right\}_{i \in I}$ allow us to reconstruct $f$ even when some of these coefficients are corrupted (see [6]).

Balan et al. [1] and Gavruta [9] established several identities and inequalities for frames in Hilbert spaces. Furuichi [8] refined Young inequalities with Specht's ratio and introduced their properties. In this paper, first we use this improved inequality in some inequalities for Parseval frames and get new inequalities. Thereafter we give improvements for general frames. However our main focus will be on Parseval frames because of their importance in applications, particularly for signal processing. Finally we give improvements for alternative dual frames too.

\section{Notation And Preliminary Results}

2.1. Frames in Hilbert space. In the definition of frame for Hilbert spaces, the optimal constants (maximal for $A$ and minimal for $B$ ) are known as the upper and lower frame bounds, respectively. If $A=B$, then this frame is called an $A$-tight frame, and if $A=B=1$, then it is called a Parseval frame. If a family of vectors $F=\left\{f_{i}\right\}_{i \in I}$ satisfies the upper bound condition (1.1), we call $F$ a Bessel family. Associated with each frame $F=\left\{f_{i}\right\}_{i \in I}$, there are three linear and bounded operators:

$T: \ell^{2}(I) \rightarrow \mathbb{H}, T x=\sum_{i \in I}\left\langle x, e_{i}\right\rangle f_{i}$ (synthesis operator);

$T^{*}: \mathbb{H} \rightarrow \ell^{2}(I), T^{*}(f)=\left\{\left\langle f, f_{i}\right\rangle\right\}_{i \in I}$, (analysis operator);

$S: \mathbb{H} \rightarrow \mathbb{H}, S f=T T^{*} f=\sum_{i \in I}\left\langle f, f_{i}\right\rangle f_{i}$ (frame operator),

where $\left\{e_{i}\right\}_{i \in I}$ is the standard orthonormal basis of $\ell^{2}(I)$. The inequalities (1.1) imply that $S$ is a (positive) self-adjoint invertible operator, and it allows reconstruction of each vector $f \in \mathbb{H}$ in terms of the family $F$ as follows:

$$
f=\sum_{i \in I}\left\langle f, S^{-1} f_{i}\right\rangle f_{i}=\sum_{i \in I}\left\langle f, f_{i}\right\rangle S^{-1} f_{i} .
$$

Then the family $\left\{\tilde{f}_{i}\right\}_{i \in I}$, where $\tilde{f}_{i}=S^{-1} f_{i}, i \in I$, is also a frame for $\mathbb{H}$ called the canonical dual frame of the $F=\left\{f_{i}\right\}_{i \in I}$. 
In general, the Bessel family $\left\{g_{i}\right\}_{i \in I}$ is called an alternative dual of the frame $F=\left\{f_{i}\right\}_{i \in I}$ if the following formula holds:

$$
f=\sum_{i \in I}\left\langle f, g_{i}\right\rangle f_{i}, \quad \text { for all } f \in \mathbb{H} .
$$

If $\left\{f_{i}\right\}_{i \in I}$ is a frame for $\mathbb{H}$ for every $J \subset I$ we define the operator

$$
S_{J} f=\sum_{i \in J}\left\langle f, f_{i}\right\rangle f_{i}
$$

and denote $J^{c}=I \backslash J$. It follows that $S=S_{J}+S_{J^{c}}$. By this definition, it is clear that if $J_{1} \subseteq J_{2}$, then $\left\|S_{J_{1}} f\right\| \leq\left\|S_{J_{2}} f\right\|$.

For more details, we refer the reader to $[2-4,10,12]$. In [1], Balan et al. proved the following identity for Parseval frames:

$$
\sum_{i \in J}\left|\left\langle f, f_{i}\right\rangle\right|^{2}-\left\|\sum_{i \in J}\left\langle f, f_{i}\right\rangle f_{i}\right\|^{2}=\sum_{i \in J^{c}}\left|\left\langle f, f_{i}\right\rangle\right|^{2}-\left\|\sum_{i \in J^{c}}\left\langle f, f_{i}\right\rangle f_{i}\right\|^{2} .
$$

Moreover, in [1] the following inequality was obtained

$$
\frac{3}{4}\|f\|^{2} \leq \sum_{i \in J}\left|\left\langle f, f_{i}\right\rangle\right|^{2}+\left\|\sum_{i \in J^{c}}\left\langle f, f_{i}\right\rangle f_{i}\right\|^{2} .
$$

See $[9,11]$ for further details. In fact, identity (2.1) was obtained as a particular case from the following result for general frames:

$$
\sum_{i \in J}\left|\left\langle f, f_{i}\right\rangle\right|^{2}+\sum_{i \in I}\left|\left\langle S_{J^{c}} f, \tilde{f}_{i}\right\rangle\right|^{2}=\sum_{i \in J^{c}}\left|\left\langle f, f_{i}\right\rangle\right|^{2}+\sum_{i \in I}\left|\left\langle S_{J} f, \tilde{f}_{i}\right\rangle\right|^{2} .
$$

Inequality (2.2) leads us to introduce, for a Parseval frame, the numbers

$$
\begin{aligned}
& v_{+}(F ; J)=\sup _{f \neq 0} \frac{\left\|\sum_{i \in J}\left\langle f, f_{i}\right\rangle f_{i}\right\|^{2}+\sum_{i \in J^{c}}\left|\left\langle f, f_{i}\right\rangle\right|^{2}}{\|f\|^{2}}, \\
& v_{-}(F ; J)=\inf _{f \neq 0} \frac{\left\|\sum_{i \in J}\left\langle f, f_{i}\right\rangle f_{i}\right\|^{2}+\sum_{i \in J^{c}}\left|\left\langle f, f_{i}\right\rangle\right|^{2}}{\|f\|^{2}} .
\end{aligned}
$$

Recall that $v_{+}(F ; J)$ is called the upper index of $F$ relative to $J$ and $v_{-}(F ; J)$ is called the lower index of $F$ relative to $J$.

Gavruta [9] presented basic properties of these indexes.

2.2. Improved Young inequality with Specht's ratio. The well-known Young inequality says that $(1-v) a+v b \geq a^{1-v} b^{v}$ for positive numbers $a, b$ and $v \in[0,1]$. A refinement of this inequality is given in the following proposition.

Lemma 2.1 ([8]). Specht's ratio

$$
R(h) \equiv \frac{h^{\frac{1}{h-1}}}{e \log h^{\frac{1}{h-1}}}, \quad h \neq 1, h>0,
$$

has the following properties:

(i) $R(1)=1$ and $R(h)=R(1 / h)>1$ for $h>0$; 
(ii) $R(h)$ is a monotone increasing function on $(1, \infty)$;

(iii) $R(h)$ is a monotone decreasing function on $(0,1)$.

Proposition 2.1 ([8]). For $a, b>0$ and $v \in[0,1]$, it follows that

$$
(1-v) a+v b \geq R\left(\left(\frac{b}{a}\right)^{r}\right) a^{1-v} b^{v}
$$

where $r \equiv \min \{v, 1-v\}$ and $R(\cdot)$ is Specht's ratio.

2.3. Operator Theory. We first state a basic result from operator theory. The following lemma appeared in [1].

Lemma 2.2. If $S, T \in L(\mathbb{H})$ satisfy $S+T=i d$, then $S-T=S^{2}-T^{2}$.

Proof. The proof follows from

$S-T=S-(i d-S)=2 S-i d=S^{2}-\left(i d-2 S+S^{2}\right)=S^{2}-(i d-S)^{2}=S^{2}-T^{2}$.

\section{Main Results}

In this section, first we improve the left-hand-side of inequality (2.2) with Specht's ratio. Thereafter in Lemma 3.1 improvements for self-adjoint operators are given, which we apply for general frames. Finally in Theorems 3.3 and 3.4, we present the results for alternate dual frames.

Theorem 3.1. If $\left\{f_{i}\right\}_{i \in I}$ is a Parseval frame for the Hilbert space $\mathbb{H}$ with frame operator $S$, then, for every $\emptyset \neq J \subset I$ and $f \in \mathbb{H}$, it follows that

$$
\left(\frac{1+2 \alpha}{2+2 \alpha}\right)\|f\|^{2} \leq \sum_{i \in J}\left|\left\langle f, f_{i}\right\rangle\right|^{2}+\left\|\sum_{i \in J^{c}}\left\langle f, f_{i}\right\rangle f_{i}\right\|^{2},
$$

where $\alpha=\inf \left\{R\left(\frac{\left\|S_{J} f\right\|}{\left\|S_{J} f\right\|}\right): f \in \mathbb{H}, J \subset I\right\}$.

Proof. Since

$$
\|f\|^{2}=\left\|S_{J} f+S_{J^{c}} f\right\|^{2} \leq\left\|S_{J} f\right\|^{2}+\left\|S_{J^{c}} f\right\|^{2}+2\left\|S_{J} f\right\|\left\|S_{J^{c}} f\right\|,
$$

by letting $v=\frac{1}{2}, a=\left\|S_{J} f\right\|$ and $b=\left\|S_{J^{c}} f\right\|$ in Proposition 2.1, we have

$$
\|f\|^{2} \leq\left(\left\|S_{J} f\right\|^{2}+\left\|S_{J^{c}} f\right\|^{2}\right)\left(1+\frac{1}{R\left(\frac{\left\|S_{J^{c}} f\right\|}{\left\|S_{J} f\right\|}\right)}\right) .
$$

Put $\alpha=\inf \left\{R\left(\frac{\left\|S_{J} f\right\|}{\left\|S_{J} f\right\|}\right): f \in \mathbb{H}, J \subset I\right\}$, then

$$
\left\langle\left(\frac{\alpha}{1+\alpha}\right) i d f, f\right\rangle \leq\left\langle\left(S_{J}^{2}+S_{J^{c}}^{2}\right) f, f\right\rangle .
$$

This implies that

$$
\left(\frac{\alpha}{1+\alpha}\right) i d \leq S_{J}^{2}+S_{J^{c}}^{2}
$$


So,

$$
\left(\frac{\alpha}{1+\alpha}+1\right) i d \leq S_{J}+S_{J^{c}}^{2}+S_{J^{c}}+S_{J}^{2}
$$

Now, by applying Lemma 2.2, it follows that

$$
\left(\frac{1+2 \alpha}{2+2 \alpha}\right) i d \leq S_{J}+S_{J^{c}}^{2}
$$

Hence,

$$
\left(\frac{1+2 \alpha}{2+2 \alpha}\right)\|f\|^{2} \leq\left\langle S_{J} f, f\right\rangle+\left\langle S_{J^{c}} f, S_{J^{c}} f\right\rangle=\sum_{i \in J}\left|\left\langle f, f_{i}\right\rangle\right|^{2}+\left\|\sum_{i \in J^{c}}\left\langle f, f_{i}\right\rangle f_{i}\right\|^{2} .
$$

Note that for $\alpha=1$, inequality (3.1) is the same as inequality (2.2) and that for $\alpha>1,(3.1)$ is an improvement of (2.2).

Corollary 3.1. Let $F=\left\{f_{i}\right\}_{i \in I}$ be a Parseval frame and let $J \subset I$. Then

$$
\frac{1+2 \alpha}{2+2 \alpha} \leq v_{-}(F ; J) \leq v_{+}(F ; J) \leq 1,
$$

where $\alpha=\inf \left\{R\left(\frac{\left\|S_{J^{c}} f\right\|}{\left\|S_{J} f\right\|}\right): f \in \mathbb{H}, J \subset I\right\}$.

Proof. By using Theorem 3.1 and the hypothesis that $F$ is a Parseval frame, we have

$$
\left(\frac{1+2 \alpha}{2+2 \alpha}\right)\|f\|^{2} \leq \sum_{i \in J}\left|\left\langle f, f_{i}\right\rangle\right|^{2}+\left\|\sum_{i \in J^{c}}\left\langle f, f_{i}\right\rangle f_{i}\right\|^{2} \leq\|f\|^{2} .
$$

So,

$$
\frac{1+2 \alpha}{2+2 \alpha} \leq \frac{\sum_{i \in J}\left|\left\langle f, f_{i}\right\rangle\right|^{2}+\left\|\sum_{i \in J^{c}}\left\langle f, f_{i}\right\rangle f_{i}\right\|^{2}}{\|f\|^{2}} \leq 1
$$

Hence,

$$
\frac{1+2 \alpha}{2+2 \alpha} \leq v_{-}(F ; J) \leq v_{+}(F ; J) \leq 1
$$

In the following lemma, we give an improvement of the inequality proved in $[9$, Theorem 2.1], under some conditions. Then we present Theorem 3.2 for general frames.

Lemma 3.1. Let $T_{1}, T_{2} \in L(\mathbb{H})$ be self-adjoint operators satisfying $T_{1}+T_{2}=i d$, such that $T_{1} \geq \frac{k}{k+1} i d$, where $k \in \mathbb{N}$ and $k>3$. Then

$$
\frac{k}{k+1}\|f\|^{2} \leq\left\langle T_{1} f, f\right\rangle+\left\|T_{2} f\right\|^{2}=\left\langle T_{2} f, f\right\rangle+\left\|T_{1} f\right\|^{2}, \quad \text { for } f \in \mathbb{H} .
$$


Proof. From our assumptions, we have

$$
\begin{aligned}
\left\langle T_{2} f, f\right\rangle+\left\|T_{1} f\right\|^{2} & =\left\langle\left(i d-T_{1}\right) f, f\right\rangle+\left\langle T_{1}^{2} f, f\right\rangle \\
& =\left\langle\left(T_{1}^{2}-T_{1}+i d\right) f, f\right\rangle \\
& =\left\langle T_{1} f, f\right\rangle+\left\langle\left(i d-T_{1}\right)^{2} f, f\right\rangle \\
& =\left\langle T_{1} f, f\right\rangle+\left\|T_{2} f\right\|^{2},
\end{aligned}
$$

and also

$$
\left\langle T_{1} f, f\right\rangle+\left\|T_{2} f\right\|^{2} \geq\left\langle\frac{k}{(k+1)} f, f\right\rangle+\left\|T_{2} f\right\|^{2} \geq \frac{k}{(k+1)}\|f\|^{2} .
$$

Remark 3.1. Notice that for $k>3$, from the inequality $\frac{3}{4}<\frac{k}{k+1}$, it follows that inequality (3.2) is an improvement of the inequality proved in [9, Theorem 2.1].

For $k=3,4,5,6,7, \ldots$, the correspondence values of $\frac{k}{k+1}$ or $1-\frac{1}{k+1}$ are $0.75<0.8<$ $0.83<0.86<0.87<\cdots$, respectively. Hence, by increasing $k$, we see that $1-\frac{1}{k+1}$ is rapidly approaching to 1 . Therefore, inequality (3.2) is better in application and we use it for frames.

Theorem 3.2. Let $\left\{f_{i}\right\}_{i \in I}$ be a frame for the Hilbert space $\mathbb{H}$ with frame operator $S$ and canonical dual frame $\left\{\tilde{f}_{i}\right\}_{i \in I}$. For every $\emptyset \neq J \subset I$, if $S^{-\frac{1}{2}} S_{J} S^{-\frac{1}{2}} \geq \frac{k}{k+1} i d$, where $k \in \mathbb{N}$ and $k \geq 3$, then

$$
\begin{aligned}
\frac{k}{k+1} \sum_{i \in I}\left|\left\langle f, f_{i}\right\rangle\right|^{2} & \leq \sum_{i \in J}\left|\left\langle f, f_{i}\right\rangle\right|^{2}+\sum_{i \in I}\left|\left\langle S_{J^{c}} f, \tilde{f}_{i}\right\rangle\right|^{2} \\
& =\sum_{i \in J^{c}}\left|\left\langle f, f_{i}\right\rangle\right|^{2}+\sum_{i \in I}\left|\left\langle S_{J} f, \tilde{f}_{i}\right\rangle\right|^{2}, \quad \text { for } f \in \mathbb{H} .
\end{aligned}
$$

Proof. For every $J \subset I$, we have $S_{J}+S_{J^{c}}=S$, and hence $S^{-\frac{1}{2}} S_{J} S^{-\frac{1}{2}}+S^{-\frac{1}{2}} S_{J^{c}} S^{-\frac{1}{2}}=$ $i d$. By our assumptions and taking $T_{1}=S^{-\frac{1}{2}} S_{J} S^{-\frac{1}{2}}, T_{2}=S^{-\frac{1}{2}} S_{J^{c}} S^{-\frac{1}{2}}$ and $S^{\frac{1}{2}} f$ instead of $f$ in Lemma 3.1, we get

$$
\frac{k}{k+1}\left\|S^{\frac{1}{2}} f\right\|^{2} \leq\left\langle S^{-\frac{1}{2}} S_{J} f, S^{\frac{1}{2}} f\right\rangle+\left\|S^{-\frac{1}{2}} S_{J^{c}} f\right\|^{2}=\left\langle S^{-\frac{1}{2}} S_{J^{c}} f, S^{\frac{1}{2}} f\right\rangle+\left\|S^{-\frac{1}{2}} S_{J} f\right\|^{2},
$$

or equivalently,

$$
\frac{k}{k+1}\langle S f, f\rangle \leq\left\langle S_{J} f, f\right\rangle+\left\langle S^{-1} S_{J^{c}} f, S_{J^{c}} f\right\rangle=\left\langle S_{J^{c}} f, f\right\rangle+\left\langle S^{-1} S_{J} f, S_{J} f\right\rangle .
$$

Therefore,

$$
\begin{aligned}
\frac{k}{k+1} \sum_{i \in I}\left|\left\langle f, f_{i}\right\rangle\right|^{2} & \leq \sum_{i \in J}\left|\left\langle f, f_{i}\right\rangle\right|^{2}+\sum_{i \in I}\left|\left\langle S_{J^{c}} f, \tilde{f}_{i}\right\rangle\right|^{2} \\
& =\sum_{i \in J^{c}}\left|\left\langle f, f_{i}\right\rangle\right|^{2}+\sum_{i \in I}\left|\left\langle S_{J} f, \tilde{f}_{i}\right\rangle\right|^{2} .
\end{aligned}
$$

For $k=3$ inequality (3.3) is the same as the inequality proved in [9, Theorem 2.2] and for $k>3$ it is an improvement for it. 
In the following, we give an improvement for alternate dual frames. We first improve an inequality given in [9] for operators under conditions.

Lemma 3.2. If $T_{1}, T_{2} \in L(\mathbb{H})$ satisfy $T_{1}+T_{2}=i d$ and $\operatorname{Re} T_{1} \geq \frac{k}{k+1} i d$, where $k \in \mathbb{N}$ and $k \geq 3$, then

$$
\frac{k}{k+1} i d \leq T_{1}^{*} T_{1}+\frac{1}{2}\left(T_{2}^{*}+T_{2}\right)=T_{2}^{*} T_{2}+\frac{1}{2}\left(T_{1}^{*}+T_{1}\right) .
$$

Proof. From our assumptions, we have

$$
\begin{aligned}
T_{1}^{*} T_{1}+\frac{1}{2}\left(T_{2}^{*}+T_{2}\right) & =T_{1}^{*} T_{1}+\frac{1}{2}\left(i d-T_{1}^{*}+i d-T_{1}\right) \\
& =T_{1}^{*} T_{1}-\frac{1}{2}\left(T_{1}^{*}+T_{1}\right)+i d \\
& =\left(i d-T_{1}^{*}\right)\left(i d-T_{1}\right)+\frac{1}{2}\left(T_{1}^{*}+T_{1}\right) \\
& =T_{2}^{*} T_{2}+\frac{1}{2}\left(T_{1}^{*}+T_{1}\right) .
\end{aligned}
$$

And also $T_{2}^{*} T_{2}+\frac{1}{2}\left(T_{1}^{*}+T_{1}\right)=T_{2}^{*} T_{2}+\operatorname{Re} T_{1} \geq \frac{k}{k+1} i d$.

Note that, for $k=3$, inequality (3.4) is the same as the inequality proved in $[9$, Theorem 3.1] and for every $k>3$, inequality (3.4) is its improvement.

Theorem 3.3. Let $\left\{f_{i}\right\}_{i \in I}$ be a frame for the Hilbert space $\mathbb{H}$ and let $\left\{g_{i}\right\}_{i \in I}$ be an alternate dual frame of $\left\{f_{i}\right\}_{i \in I}$. For every $J \subset I$ and $f \in \mathbb{H}$, if $\operatorname{Re}\left\langle\left(\sum_{i \in J}\left\langle f, g_{i}\right\rangle f_{i}\right), f\right\rangle \geq$ $\frac{k}{k+1}\langle f, f\rangle$, where $k \in \mathbb{N}$ and $k \geq 3$, then

$$
\begin{aligned}
\frac{k}{k+1}\|f\|^{2} & \leq \operatorname{Re} \sum_{i \in J}\left\langle f, g_{i}\right\rangle \overline{\left\langle f, f_{i}\right\rangle}+\left\|\sum_{i \in J^{c}}\left\langle f, g_{i}\right\rangle f_{i}\right\|^{2} \\
& =\operatorname{Re} \sum_{i \in J^{c}}\left\langle f, g_{i}\right\rangle \overline{\left\langle f, f_{i}\right\rangle}+\left\|\sum_{i \in J}\left\langle f, g_{i}\right\rangle f_{i}\right\|^{2}, \quad \text { for } f \in \mathbb{H} .
\end{aligned}
$$

Proof. For every $J \subset I$, define the bounded linear operator $Z_{J}$ on $\mathbb{H}$ by

$$
Z_{J} f:=\sum_{i \in J}\left\langle f, g_{i}\right\rangle f_{i}
$$

By the Cauchy-Schwartz inequality, it follows that this series converges unconditionally. Since $Z_{J}+Z_{J^{c}}=i d$, by Lemma 3.1, for every $f \in \mathbb{H}$, we have

$$
\begin{aligned}
\frac{k}{k+1}\langle f, f\rangle & \leq\left\langle Z_{J}^{*} Z_{J} f, f\right\rangle+\frac{1}{2}\left\langle\left(Z_{J^{c}}^{*}+Z_{J^{c}}\right) f, f\right\rangle \\
& =\left\langle Z_{J^{c}}^{*} Z_{J^{c}} f, f\right\rangle+\frac{1}{2}\left\langle\left(Z_{J}^{*}+Z_{J}\right) f, f\right\rangle
\end{aligned}
$$


or

$$
\begin{aligned}
\frac{k}{k+1}\|f\|^{2} & \leq\left\|K_{J} f\right\|^{2}+\frac{1}{2}\left(\overline{\left\langle Z_{J^{c}} f, f\right\rangle}+\left\langle Z_{J^{c}} f, f\right\rangle\right) \\
& =\left\|Z_{J^{c}} f\right\|^{2}+\frac{1}{2}\left(\overline{\left\langle Z_{J} f, f\right\rangle}+\left\langle Z_{J} f, f\right\rangle\right) .
\end{aligned}
$$

Hence,

$$
\begin{aligned}
\frac{k}{k+1}\|f\|^{2} & \leq\left\|\sum_{i \in J}\left\langle f, g_{i}\right\rangle f_{i}\right\|^{2}+\operatorname{Re}\left\langle\sum_{i \in J^{c}}\left\langle f, g_{i}\right\rangle f_{i}, f\right\rangle \\
& =\left\|\sum_{i \in J^{c}}\left\langle f, g_{i}\right\rangle f_{i}\right\|^{2}+\operatorname{Re}\left\langle\sum_{i \in J}\left\langle f, g_{i}\right\rangle f_{i}, f\right\rangle,
\end{aligned}
$$

and the proof is completed.

Note that, for $k=3$, inequality $(3.5)$ is the same as the inequality proved in $[9$, Theorem 3.2] and for every $k>3$, inequality (3.5) is its improvement. Finally, we give a more general result.

Theorem 3.4. Let $\left\{f_{i}\right\}_{i \in I}$ be a frame for the Hilbert space $\mathbb{H}$ and let $\left\{g_{i}\right\}_{i \in I}$ be an alternate dual frame of $\left\{f_{i}\right\}_{i \in I}$. For every $f \in \mathbb{H}$, if $\operatorname{Re}\left\langle\left(\sum_{i \in J}\left\langle f, g_{i}\right\rangle f_{i}\right), f\right\rangle \geq \frac{k}{k+1}\langle f, f\rangle$, where $k \in \mathbb{N}$ and $k \geq 3$, then for any bounded sequence $\left\{w_{i}\right\}_{i \in I}$, we have

$$
\begin{aligned}
\frac{k}{k+1}\|f\|^{2} & \leq \operatorname{Re} \sum_{i \in I} w_{i}\left\langle f, g_{i}\right\rangle \overline{\left\langle f, f_{i}\right\rangle}+\left\|\sum_{i \in I}\left(1-w_{i}\right)\left\langle f, g_{i}\right\rangle f_{i}\right\|^{2} \\
& =\operatorname{Re} \sum_{i \in I}\left(1-w_{i}\right)\left\langle f, g_{i}\right\rangle \overline{\left\langle f, f_{i}\right\rangle}+\left\|\sum_{i \in I} w_{i}\left\langle f, g_{i}\right\rangle f_{i}\right\|^{2}, \quad \text { for } f \in \mathbb{H} .
\end{aligned}
$$

Proof. In Lemma 3.2, we put

$$
T_{1} f=\sum_{i \in I} w_{i}\left\langle f, g_{i}\right\rangle f_{i}, \quad T_{2} f=\sum_{i \in I}\left(1-w_{i}\right)\left\langle f, g_{i}\right\rangle f_{i} .
$$

The result is obtained from Theorem 3.3, if we take $J \subset I$ and

$$
w_{i}= \begin{cases}1, & \text { for } i \in J \\ 0, & \text { for } i \in J^{c}\end{cases}
$$

\section{REFERENCES}

[1] R. Balan, P. G. Casazza, D. Edidin and G. Kutyniok, A new identity for Parseval frames, Proc. Amer. Math. Soc. 135 (2007), 1007-1015.

[2] P. G. Casazza, The art of frame theory, Taiwanese J. Math. 4 (2000), 129-201.

[3] P. G. Casazza and G. Kutyniok, Frames of subspaces, in: Wavelets, Frames and Operator theory, Contemp. Math. 345, Amer. Math. Soc., Providence, RI, 2004, 87-113,.

[4] O. Christensen, An Introduction to Frames and Riesz Bases, Birkhauser/Springer, Basel, 2016.

[5] I. Daubechies, Ten Lectures on Wavelets, SIAM, Philadelphia, PA, 1992.

[6] J. Duffin and A. C. Schaeffer, A class of nonharmonic Fiurier series, Trans. Amer. Math. Soc. 72 (1952), 341-366. 
[7] Y. C. Eldar and G. D. Forney, Optimal tight frames and quantum measurement, IEEE Trans. Inform. Theory 48 (2002), 599-610.

[8] Sh. Furuichi, Refined Young inequalities with Specht's ratio, J. Egyptian Math. Soc. 20 (2012), 46-49.

[9] P. Gavruta, On some identities and inequalities for frames in Hilbert spaces, J. Math. Anal. Appl. 321 (2006), 469-478.

[10] L. Gavruta, Frames for operators, Appl. Comput. Harmon. Anal. 32 (2012), 139-144.

[11] Q. P. Guo, J. S. Leng and H. B. Li, Some equalities and inequalities for fusion frames, Springer Plus 5 (2016), Article ID 121, 10 pages.

[12] D. Han and D. R. Larson, Frames, bases and group representations, Mem. Amer. Math. Soc. 147 (2000), 94 pages.

[13] R. Vale and S. Waldron, Tight frames and their symmetries, Constr. Approx. 21 (2005), 83-112.

\author{
${ }^{1}$ Department of Mathematics, \\ Mashhad Branch, Islamic Azad University, \\ MASHHAD, IRAN \\ Email address: fsultanzadeh@gmail.com \\ Email address: mhassanimath@gmail.com \\ Email address: math.erfanian@gmail.com \\ ${ }^{2}$ Department of Pure Mathematics, \\ FERDOWSI UNIVERSITY OF MASHHAD, \\ MASHHAD, IRAN \\ Email address: kamyabi@um.ac.ir
}



Kragujevac Journal of Mathematics

Volume 46(1) (2022), Pages 49-64.

\title{
SOLUTION SET FOR IMPULSIVE FRACTIONAL DIFFERENTIAL INCLUSIONS
}

\author{
MOUSTAFA BEDDANI ${ }^{1}$
}

\begin{abstract}
This paper aims to an initial value problem for an impulsive fractional differential inclusion with the Riemann-Liouville fractional derivative. We apply Covitz and Nadler theorem concerning the study of the fixed point for multivalued maps to obtain the existence results for the given problems. We also obtain some topological properties about the solution set.
\end{abstract}

\section{INTRODUCTION}

We study the existence of solutions and determine certain topological properties of the solutions set for the following impulsive fractional differential inclusion:

$$
\left\{\begin{array}{l}
{ }^{R L} D^{\alpha} y(t) \in F(t, y(t)) \quad \text { a.e. } t \in J=(0, T], t \neq t_{k}, \\
\lim _{t \rightarrow 0^{+}} t^{1-\alpha} y(t)=c \\
\left.\Delta^{*} y\right|_{t_{k}}=I_{k}\left(y\left(t_{k}^{-}\right)\right)
\end{array}\right.
$$

where $k=1, \ldots, m, 0<\alpha \leq 1,{ }^{R L} D^{\alpha}$ is the Riemann-Liouville fractional derivative, $F: J \times \mathbb{R} \rightarrow\{X \subset \mathbb{R}: X \neq \emptyset\}$ is a given multivalued function, $c \in \mathbb{R}, I_{k}: \mathbb{R} \rightarrow \mathbb{R}$ are continuous functions, $0=t_{0}<t_{1}<\cdots<t_{m}<t_{m+1}=T$ and $\left.\Delta^{*} y\right|_{t_{k}}=y^{*}\left(t_{k}^{+}\right)-y\left(t_{k}^{-}\right)$, where $y^{*}\left(t_{k}^{+}\right)=\lim _{t \rightarrow t_{k}^{+}}\left(t-t_{k}\right)^{1-\alpha} y(t)$ and $y\left(t_{k}^{-}\right)=\lim _{t \rightarrow t_{k}^{-}} y(t)$.

More precisely, we present an overall existence result for (1.1) by using Covitz and Nadler fixed-point theorem for multivalued maps. Afterwards, we prove the compactness and acyclicity of the solution set for this problem.

Key words and phrases. Impulsive fractional differential inclusions, Riemann-Liouville fractional derivative, fixed point, solution set, compactness, contractible.

2010 Mathematics Subject Classification. Primary: 34A60, 34A08 Secondary: 34A37.

DOI 10.46793/KgJMat2201.049B

Received: March 25, 2019.

Accepted: August 19, 2019. 
Since the 1960s, the subject of functional differential inclusions and impulsive ordinary differential inclusions with various conditions have been investigated by many authors $[1,2,8,11,14,24-26,32,35,39,40]$, and has several applications in different areas as engineering, electrical, networks electrochemistry, fluid flow, etc. For more details we refer the reader to see the following references $[4,13,20,21,30,31,34,36]$.

The topological and geometric properties of the solution set for differential inclusions are examined by many mathematicians (see for example $[3,10,16,17,22,37]$ ) where the concept of quasi-concavity is extended to multifunctions contractibility, absolutely retract, acyclicity, $R_{\delta}$-sets properties are given.

This work is structured as follows: in the second section, we recall some definitions and properties that are needed throughout this article. Afterwards, in the third section, we show that the solution sets is contractible to a point. Finally, we give an example which illustrate the principle result of this paper.

\section{Preliminary Results}

The object of this section is to recall some basic definitions and useful notations in multivalued analysis. Let $C([a, b], \mathbb{R})$ be the Banach space of all continuous functions $h$ from the interval $[a, b]$ into $\mathbb{R}$ with the norm

$$
\|h\|_{\infty}=\sup _{t \in[a, b]}|h(t)|
$$

and $L^{1}([a, b], \mathbb{R})$ the Banach space of all Lebesgue integrable functions $h$ from the interval $[a, b]$ into $\mathbb{R}$ with the norm

$$
\|h\|_{L^{1}}=\int_{a}^{b}|h(t)| d t .
$$

For a given metric space $E$, we denote:

- $\mathcal{P}(E)=\{X \subset E: X \neq \emptyset\}$;

- $\mathcal{P}_{c l}(E)=\{X \in \mathcal{P}(E): X$ is closed $\}$

- $\mathcal{P}_{b}(E)=\{X \in \mathcal{P}(E): X$ is bounded $\}$;

- $\mathcal{P}_{c p}(E)=\{X \in \mathcal{P}(E): X$ is compact $\}$;

- $\mathcal{P}_{c}(E)=\{X \in \mathcal{P}(E): X$ is convex $\}$;

- $\mathcal{P}_{c p, c}(E)=\mathcal{P}_{c p}(E) \cap \mathcal{P}_{c}(E)$.

If $X$ and $Y$ are two subsets of the metric space $E$, and $x$ (resp. $y$ ) is a point in $X$ (resp. $Y$ ), we denote:

$$
d(x, Y)=\inf _{y \in Y} d(x, y) \quad \text { and } \quad d(X, y)=\inf _{x \in X} d(x, y) .
$$

Recall that, the Hausdorff pseudo-metric distance $H_{d}$ on $\mathcal{P}(E)$ defined by

$$
H_{d}(X, Y):=\max \left\{\sup _{x \in X} d(x, Y), \sup _{y \in Y} d(X, y)\right\} .
$$


Form the previous definition, it is well-known that $\left(\mathcal{P}_{c l, b}(E), H_{d}\right)\left(\operatorname{resp} .\left(\mathcal{P}_{c l}(E), H_{d}\right)\right)$ is a metric space (resp. is a generalized metric space).

Definition 2.1. Let $M: \rightarrow \mathcal{P}(E)$ be a multivalued map.

(a) We say that $M$ is $\gamma$-Lipschitz if there exists a positive real number $\gamma$ such that

$$
H_{d}(M(x), M(y)) \leq \gamma d(x, y), \quad \text { for all } x, y \in E .
$$

(b) The map $M$ is called a contraction if it is $\gamma$-Lipschitz for some $0<\gamma<1$.

Notice that, if $M$ is $\gamma$-Lipschitz on a Banach space $E$, then for every real number $\gamma^{\prime}$ greater than $\gamma, M(x) \subset M(y)+\gamma^{\prime} d(x, y) B(0,1)$, where $B(0,1)$ is the unit ball of $E$.

Definition 2.2. Let $G: X \rightarrow \mathcal{P}_{c l}(Y)$ be a multivalued map, where $X$ and $Y$ are two metric spaces.

(a) We say that $G$ is closed valued (resp. convex valued) if $G(x)$ is closed (resp. convex) for all $x \in E$.

(b) Every single-valued map $g: X \rightarrow Y$ is called a selection of $G$. We write $g \subset G$ whenever $g(x) \in G(x)$ for all $x \in X . G: J \times \mathbb{R} \rightarrow \mathcal{P}(\mathbb{R})$, we define the set of selections of $G$ by

$$
S_{G, x}=\left\{v \in L^{1}(J, \mathbb{R}): v(t) \in G(t, x(t)) \text { a.e. } t \in J\right\} .
$$

Definition 2.3. A multivalued map $G: J \times \mathbb{R} \rightarrow \mathcal{P}(\mathbb{R})$ is called $L^{1}$-Carathéodory if the following are satisfied:

(a) the function $G(\cdot, x)$ is measurable for each $x \in \mathbb{R}$;

(b) the function $G(t, \cdot)$ is upper semi-continuous for almost all $t \in J$;

(c) for every positive real $r$, there exists a function $f_{r} \in L^{1}\left(J, \mathbb{R}_{+}\right)$such that

$\|G(t, x)\|=\sup \{|v|: v \in G(t, x)\} \leq f_{r}(t)$ a.e. $t \in J$ and for all $x \in[-r, r]$.

For more details about multivalued analysis, we refer the reader to see $[5-7,12,23$, 27-29].

Below we present the definition of contractible spaces, and for details about this type of spaces, we recommend $[5,10,15,33]$.

Definition 2.4. A contractible subset of a Banach space $X$ is a nonempty subset $A$ of $X$ for which there exists a continuous homotopy $\psi: A \times[0,1] \rightarrow A$ and $a_{0} \in A$ such that for all $a \in A, \psi(a, 0)=a$ and $\psi(a, 1)=a_{0}$.

We give now two basic definitions used frequently in fractional computation theory. Definition 2.5 ([18,19]). Let $h \in L^{1}\left([a, b], \mathbb{R}_{+}\right)$. The fractional order integral of $h$ is given by

$$
I_{a}^{\alpha} h(t)=\int_{a}^{t} \frac{(t-s)^{\alpha-1}}{\Gamma(\alpha)} h(s) d s .
$$


Definition 2.6 ([19]). Let $b$ be a positive real number and $h$ a real function defined on the interval $[0, b]$. The Riemann-Liouville fractional derivative of order $\alpha \in \mathbb{R}_{+}$of $h$ is defined as follows:

$$
{ }^{R L} D_{0^{+}}^{\alpha} h(t)=\frac{1}{\Gamma(n-\alpha)} \frac{d^{n}}{d t^{n}}\left(\int_{0}^{t}(t-s)^{n-\alpha-1} h(s) d s\right) .
$$

\section{Main Results}

Let

$$
\begin{aligned}
P C_{*}([0, T], \mathbb{R})= & \left\{y:[0, T] \longrightarrow \mathbb{R}: y_{k} \in C\left(t_{k}, t_{k+1}\right], k=0, \ldots, m,\right. \text { and there } \\
& \text { exist } \left.y\left(t_{k}^{-}\right), y_{*}\left(t_{k}^{+}\right), k=1, \ldots, m \text {, with } y\left(t_{k}\right)=y\left(t_{k}^{-}\right)\right\} .
\end{aligned}
$$

It is known that this set is Banach space with the norm

$$
\|y\|_{P C_{*}}=\max _{k=1, \ldots m}\left\|y_{k}\right\|_{*},
$$

where $y_{k}$ is the restriction of $y$ to the interval $J_{k}=\left(t_{k}, t_{k+1}\right]$ for every $k=0, \ldots, m$, and

$$
\left\|y_{k}\right\|_{*}=\sup _{t \in\left[t_{k}, t_{k+1}\right]}\left|\left(t-t_{k}\right)^{1-\alpha} y_{k}(t)\right|, \quad \text { for every } k=1, \ldots, m .
$$

When $A \subset P C_{*}([0, T], \mathbb{R})$, we define $\mathcal{A}_{\alpha}$ by

$$
\mathcal{A}_{\alpha}=\left\{y_{\alpha}: y \in A\right\}
$$

where

$$
y_{\alpha}(t)= \begin{cases}\left(t-t_{k}\right)^{1-\alpha} y(t), & \text { if } t \in\left(t_{k}, t_{k}+1\right], \\ \lim _{t \rightarrow t_{k}}\left(t-t_{k}\right)^{1-\alpha} y(t), & \text { if } t=t_{k} .\end{cases}
$$

Theorem 3.1. Let $A$ be a bounded subset in the Banach space $P C_{*}([0, T], \mathbb{R})$ such that $\mathcal{A}_{\alpha}$ is equicontinuous on $P C([0, T], \mathbb{R})$. Then the set $A$ is relatively compact in $P C_{*}([0, T], \mathbb{R})$.

Proof. We know that, if $\left\{y_{n}\right\}_{n=1}^{\infty} \subset A$, then $\left\{\left(y_{\alpha}\right)_{n}\right\}_{n=1}^{\infty} \subset P C([0, T], \mathbb{R})$. From ArzelaAscoli theorem, the set $K_{0}=\left\{\left(y_{\alpha}\right)_{n}: n \in \mathbb{N}^{*}\right\}$ is relatively compact in the space $P C([0, T], \mathbb{R})$. So, we can find a subsequence of $\left(y_{\alpha}\right)_{n \in \mathbb{N}}$ still denoted by the same indices such that $\lim y_{n} \in\left(P C([0, T], \mathbb{R}),\|\cdot\|_{P C}\right)$. Put $y=\lim y_{n}$. We have

$$
\left.\left\|\left(y_{\alpha}\right)_{n}-y\right\|_{*}=\sup _{t \in\left[t_{k}, t_{k+1}\right]}\left(t-t_{k}\right)^{1-\alpha} \mid y_{\alpha}\right)_{n}(t)-y(t) \mid \rightarrow 0, \quad n \rightarrow+\infty .
$$

So, $y_{n} \rightarrow y, n \rightarrow+\infty$, on $P C_{*}([0, T], \mathbb{R})$.

To explain our results, we need the following lemmas. 
Lemma 3.1 ([38]). Let $\alpha$ be a positive real number and $n=1+[\alpha]$. Then the following differential equation ${ }^{R L} D_{a^{+}}^{\alpha} h(t)=0$, has solutions of the form $h(t)=\sum_{k=1}^{n} c_{k}(t-a)^{\alpha-k}$ for some real numbers $c_{1}, c_{2}, \ldots, c_{n}$.

Lemma $3.2([38])$. Let $\alpha$ be a positive real number and $n=1+[\alpha]$. Then there exist some real numbers $c_{1}, c_{2}, \ldots, c_{n}$ such that

$$
I^{\alpha R L} D_{a^{+}}^{\alpha} h(t)=h(t)+\sum_{k=1}^{n} c_{k}(t-a)^{\alpha-k} .
$$

Lemma 3.3. Let $\alpha$ be a real number in the interval $(0,1)$ and $h$ a continuous function. If $y$ is a solution of following problem

$$
\begin{aligned}
{ }^{R L} D^{\alpha} y(t) & =h(t), \quad \text { for all } t \in J \text { and } t \neq t_{m} \text { for all } k=1, \ldots, m, \\
\left.\Delta^{*} y\right|_{t_{k}} & =I_{k}\left(y\left(t_{k}^{-}\right)\right), \quad \text { for all } k=1, \ldots, m, \\
\lim _{t \rightarrow 0} t^{1-\alpha} y(t) & =c
\end{aligned}
$$

Then

$$
y(t)= \begin{cases}t^{\alpha-1} c+\frac{1}{\Gamma(\alpha)} \int_{0}^{t}(t-s)^{\alpha-1} h(s) d s, & \text { if } 0 \leq t \leq t_{1}, \\ \left(t-t_{1}\right)^{\alpha-1} t_{1}^{\alpha-1} c+\frac{\left(t-t_{1}\right)^{\alpha-1}}{\Gamma(\alpha)} \int_{0}^{t_{1}}\left(t_{1}-s\right)^{\alpha-1} h(s) d s & \\ +\frac{\left(t-t_{1}\right)^{\alpha-1}}{\Gamma(\alpha)} I_{1}\left(y\left(t_{1}^{-}\right)\right)+\frac{1}{\Gamma(\alpha)} \int_{t_{1}}^{t}(t-s)^{\alpha-1} h(s) d s, & \text { if } t_{1}<t \leq t_{2}, \\ \left(t-t_{k}\right)^{\alpha-1} \prod_{i=1}^{k}\left(t_{i}-t_{i-1}\right)^{\alpha-1} c & \\ +\frac{\left(t-t_{k}\right)^{\alpha-1}}{\Gamma(\alpha)}\left[\int_{t_{k-1}}^{t_{k}}\left(t_{k}-s\right)^{\alpha-1} h(s) d s+\right. & \\ \left.\sum_{i=1}^{k-1} \prod_{j=1}^{k-i}\left(t_{k-j+1}-t_{k-j}\right)^{\alpha-1} \int_{t_{i-1}}^{t_{i}}\left(t_{i}-s\right)^{\alpha-1} h(s) d s\right] & \\ +\frac{\left(t-t_{k}\right)^{\alpha-1}}{\Gamma(\alpha)}\left[I_{k}\left(y\left(t_{k}^{-}\right)\right)\right. & \\ \left.+\sum_{i=1}^{k-1} \prod_{j=1}^{k-i}\left(t_{k-j+1}-t_{k-j}\right)^{\alpha-1} I_{i}\left(y\left(t_{i}^{-}\right)\right)\right] & \\ +\frac{1}{\Gamma(\alpha)} \int_{t_{k}}^{t}(t-s)^{\alpha-1} h(s) d s, & \text { if } t_{k}<t \leq t_{k+1}, \\ & 2 \leq k \leq m .\end{cases}
$$

Proof. Suppose that $y$ satisfies (3.1)-(3.3). It is clear when $0 \leq t \leq t_{1}$, we have

$$
{ }^{R L} D^{\alpha} y(t)=h(t) \text {. }
$$


From the previous lemma, we get

$$
y(t)=t^{\alpha-1} c_{1}+\frac{1}{\Gamma(\alpha)} \int_{0}^{t}(t-s)^{\alpha-1} h(s) d s .
$$

Hence, $c_{1}=c$. Thus,

$$
y(t)=t^{\alpha-1} c+\frac{1}{\Gamma(\alpha)} \int_{0}^{t}(t-s)^{\alpha-1} h(s) d s .
$$

When $t_{1}<t \leq t_{2}$, we can obtain, by using the previous lemma, that

$$
\begin{aligned}
y(t)= & \left(t-t_{1}\right)^{\alpha-1} y^{*}\left(t_{1}^{+}\right)+\frac{1}{\Gamma(\alpha)} \int_{t_{1}}^{t}(t-s)^{\alpha-1} h(s) d s \\
= & \left(t-t_{1}\right)^{\alpha-1}\left(I_{1}\left(y\left(t_{1}^{-}\right)+y\left(t_{1}^{-}\right)\right)+\frac{1}{\Gamma(\alpha)} \int_{t_{1}}^{t}(t-s)^{\alpha-1} h(s) d s\right. \\
= & \left(t-t_{1}\right)^{\alpha-1} t_{1}^{\alpha-1} c+\frac{\left(t-t_{1}\right)^{\alpha-1}}{\Gamma(\alpha)} \int_{0}^{t_{1}}\left(t_{1}-s\right)^{\alpha-1} h(s) d s \\
& +\frac{1}{\Gamma(\alpha)} \int_{t_{1}}^{t}(t-s)^{\alpha-1} h(s) d s+\left(t-t_{1}\right)^{\alpha-1} I_{1}\left(y\left(t_{1}^{-}\right)\right) .
\end{aligned}
$$

If $t_{2}<t \leq t_{3}$, Lemma 3.2 implies

$$
\begin{aligned}
y(t)= & \left(t-t_{2}\right)^{\alpha-1} y^{*}\left(t_{2}^{+}\right)+\frac{1}{\Gamma(\alpha)} \int_{t_{2}}^{t}(t-s)^{\alpha-1} h(s) d s \\
y(t)= & \left(t-t_{2}\right)^{\alpha-1}\left[y\left(t_{2}^{-}\right)+I_{2}\left(y\left(t_{2}^{-}\right)\right)\right]+\frac{1}{\Gamma(\alpha)} \int_{t_{2}}^{t}(t-s)^{\alpha-1} h(s) d s \\
= & \left(t-t_{2}\right)^{\alpha-1}\left(t_{2}-t_{1}\right)^{\alpha-1} t_{1}^{\alpha-1}+\frac{\left(t-t_{2}\right)^{\alpha-1}\left(t_{2}-t_{1}\right)^{\alpha-1}}{\Gamma(\alpha)} \int_{0}^{t_{1}}\left(t_{1}-s\right)^{\alpha-1} h(s) d s \\
& +\frac{\left(t-t_{2}\right)^{\alpha-1}}{\Gamma(\alpha)} \int_{t_{1}}^{t_{2}}\left(t_{2}-s\right)^{\alpha-1} h(t) d s+\frac{1}{\Gamma(\alpha)} \int_{t_{2}}^{t}(t-s)^{\alpha-1} h(s) d s \\
& +\left(t-t_{2}\right)^{\alpha-1}\left[\left(t_{2}-t_{1}\right)^{\alpha-1} I_{1}\left(y\left(t_{1}^{-}\right)\right)+I_{2}\left(y_{2}\left(t_{2}^{-}\right)\right)\right] .
\end{aligned}
$$

Finally, when $t_{k}<t \leq t_{k+1}$, we obtain (3.4), by using Lemma 3.2.

Definition 3.1. Let $y$ be a function in $P C_{*}$. We say that $y$ is a mild solution of the problem (1.1) if there exists $v \in L^{1}(J, \mathbb{R})$ such that $v(t) \in F(t, y(t))$ a.e. on $J \backslash$ $\left\{t_{1}, \ldots, t_{m}\right\}, \lim _{t \longrightarrow 0} t^{1-\alpha} y(t)=c,\left.\Delta^{*} y\right|_{t_{k}}=I_{k}\left(t_{k}^{-}\right)$for all $k=1, \ldots, m$, and ${ }^{R L} D^{\alpha} y(t)=$ $v(t)$ for all $t \in J \backslash\left\{t_{1}, \ldots, t_{m}\right\}$.

Definition $3.2([6,23])$. A single-valued map $f:[0, a] \times X \rightarrow Y$ be a single-valued map is said to be measurable locally Lipschitz (mLL) if

(1) $f(\cdot, x)$ is measurable for every $x \in X$, and

(2) for each $x \in X$, there exists a neighborhood $V_{x}$ of $x$ and an integrable function $L_{x}:[0, a] \rightarrow[0, \infty)$ such that

$$
d^{\prime}\left(f\left(t, x_{1}\right), f\left(t, x_{2}\right)\right) \leq L_{x}(t) d\left(x_{1}, x_{2}\right) \quad \text { a.e. } t \in[0, a] \text { and } x_{1}, x_{2} \in V_{x} .
$$


Definition 3.3 ([6, 23]). A mapping $F:[0, a] \times X \rightarrow \mathcal{P}(Y)$ is mLL-selectionable provided there exists a measurable locally-Lipchitzian map $f:[0, a] \times X \rightarrow Y$ such that $f \in F$.

Lemma $3.4([2,11])$. If $N: X \rightarrow \mathcal{P}_{c l}(X)$ is a contraction on a complete metric space $X$, then the fixed point set of $N$ is nonempty.

Theorem 3.2. Let $F: J \times \mathbb{R} \rightarrow \mathcal{P}_{c p}(\mathbb{R})$ be a mLL-selectionable multivalued map such that the following properties are satisfied:

$\left(H_{1}\right)$ there exist $\bar{a}$ and $\bar{b}$ in $\mathbb{R}_{+}$such that for every $x \in \mathbb{R}$, we have

$$
\|F(t, x)\|_{\mathcal{P}} \leq \bar{a}|x|+\bar{b} \quad \text { a.e. } t \in J
$$

$\left(H_{2}\right)$ there exist $a_{k}$ and $b_{k} \in \mathbb{R}_{+}$such that

$$
\left|I_{k}(x)\right| \leq a_{k}|x|+b_{k}, \quad \text { for } x \in \mathbb{R} ;
$$

$\left(H_{3}\right)$ there exist $p \in C\left([0, T], \mathbb{R}_{+}\right)$such that

$$
H_{d}\left(F\left(t, z_{1}\right), F\left(t, z_{2}\right)\right) \leq p(t)\left\|z_{1}-z_{2}\right\|, \quad \text { for all } z_{1}, z_{2} \in \mathbb{R},
$$

and $d(0, F(t, 0)) \leq p(t), t \in J$;

$\left(H_{4}\right)$ there exist a real number $L \in \mathbb{R}_{+}$such that

$$
\left|I_{k}\left(z_{1}\right)-I_{k}\left(z_{2}\right)\right| \leq L\left\|z_{1}-z_{2}\right\|, \quad \text { for all } z_{1}, z_{2} \in \mathbb{R} .
$$

If

$$
\frac{T^{\alpha}\|p\|_{\infty} \Gamma(\alpha)\left(1+m T^{\alpha-1}\right)}{\Gamma(2 \alpha)}+\frac{m T_{0}^{\alpha-1} L}{\Gamma(\alpha)}<1
$$

then (1.1) has a solution. In addition, if $F: J \times \mathbb{R} \rightarrow \mathcal{P}_{c p}(\mathbb{R})$ is a Carathéodory multivalued map with compact convex values, then the solution set is contractible and compact, and hence it is acyclic.

Proof. Step 1. Existence of solutions. Let $P: P C_{*} \longrightarrow \mathcal{P}\left(P C_{*}\right)$ the operator defined by

$$
\begin{aligned}
P(y)= & \left\{h \in P C_{*}: h(t)=\left(t-t_{k}\right)^{\alpha-1} \prod_{i=1}^{k}\left(t_{i}-t_{i-1}\right)^{\alpha-1} c\right. \\
& +\frac{\left(t-t_{k}\right)^{\alpha-1}}{\Gamma(\alpha)}\left[\int_{t_{k-1}}^{t_{k}}\left(t_{k}-s\right)^{\alpha-1} h(s) d s\right. \\
& \left.+\sum_{i=1}^{k-1} \prod_{j=1}^{k-i}\left(t_{k-j+1}-t_{k-j}\right)^{\alpha-1} \int_{t_{i-1}}^{t_{i}}\left(t_{i}-s\right)^{\alpha-1} v(s) d s\right]+\frac{\left(t-t_{k}\right)^{\alpha-1}}{\Gamma(\alpha)}\left[I_{k}\left(y\left(t_{k}^{-}\right)\right)\right. \\
& \left.\left.+\sum_{i=1}^{k-1} \prod_{j=1}^{k-i}\left(t_{k-j+1}-t_{k-j}\right)^{\alpha-1} I_{i}\left(y\left(t_{i}^{-}\right)\right)\right]+\frac{1}{\Gamma(\alpha)} \int_{t_{k}}^{t}(t-s)^{\alpha-1} v(s) d s\right\},
\end{aligned}
$$

where $v \in S_{F, y}=\left\{v \in L^{1}(J, \mathbb{R}): v(t) \in F(t, y(t))\right.$ a.e. $\left.t \in J\right\}$. Now, we show that the operator $F$ satisfies the hypotheses $\left(H_{1}\right),\left(H_{2}\right)$ and $\left(H_{3}\right)$ of Lemma 3.4. To prove 
that $P(y) \in \mathcal{P}_{c l}\left(P C_{*}([0, T], \mathbb{R})\right)$ for all $y \in P C_{*}([0, T], \mathbb{R})$, let $\left\{u_{n}\right\}_{n=0}^{\infty} \in F(y)$ be a sequence converges to $u$ on the space $P C_{*}([0, T], \mathbb{R})$. Then $u \in P C_{*}([0, T], \mathbb{R})$ and there exists $v_{n} \in S_{F, y}$ such that, for each $t \in(0, T]$

$$
\begin{aligned}
u_{n}(t)= & \left(t-t_{k}\right)^{\alpha-1} \prod_{i=1}^{k}\left(t_{i}-t_{i-1}\right)^{\alpha-1} c \\
& +\frac{\left(t-t_{k}\right)^{\alpha-1}}{\Gamma(\alpha)}\left[\int_{t_{k-1}}^{t_{k}}\left(t_{k}-s\right)^{\alpha-1} v_{n}(s) d s\right. \\
& \left.+\sum_{i=1}^{k-1} \prod_{j=1}^{k-i}\left(t_{k-j+1}-t_{k-j}\right)^{\alpha-1} \int_{t_{i-1}}^{t_{i}}\left(t_{i}-s\right)^{\alpha-1} v_{n}(s) d s\right] \\
& +\frac{\left(t-t_{k}\right)^{\alpha-1}}{\Gamma(\alpha)}\left[I_{k}\left(y\left(t_{k}^{-}\right)\right)+\sum_{i=1}^{k-1} \prod_{j=1}^{k-i}\left(t_{k-j+1}-t_{k-j}\right)^{\alpha-1} I_{i}\left(y\left(t_{i}^{-}\right)\right)\right] \\
& +\frac{1}{\Gamma(\alpha)} \int_{t_{k}}^{t}(t-s)^{\alpha-1} v_{n}(s) d s
\end{aligned}
$$

We use the fact that $F$ has compact value and by passing (if necessary) onto a subsequence to obtain that $v_{n}$ converges to $v$ in $L^{1}([0, T], \mathbb{R})$, we get $v \in S_{F, y}$, and for each $t \in(0, T]$, we have

$$
\begin{aligned}
u_{n}(t) \rightarrow u(t)= & \left(t-t_{k}\right)^{\alpha-1} \prod_{i=1}^{k}\left(t_{i}-t_{i-1}\right)^{\alpha-1} c \\
& +\frac{\left(t-t_{k}\right)^{\alpha-1}}{\Gamma(\alpha)}\left[\int_{t_{k-1}}^{t_{k}}\left(t_{k}-s\right)^{\alpha-1} v(s) d s\right. \\
& \left.+\sum_{i=1}^{k-1} \prod_{j=1}^{k-i}\left(t_{k-j+1}-t_{k-j}\right)^{\alpha-1} \int_{t_{i-1}}^{t_{i}}\left(t_{i}-s\right)^{\alpha-1} v(s) d s\right] \\
& +\frac{\left(t-t_{k}\right)^{\alpha-1}}{\Gamma(\alpha)}\left[I_{k}\left(y\left(t_{k}^{-}\right)\right)+\sum_{i=1}^{k-1} \prod_{j=1}^{k-i}\left(t_{k-j+1}-t_{k-j}\right)^{\alpha-1} I_{i}\left(y\left(t_{i}^{-}\right)\right)\right] \\
& +\frac{1}{\Gamma(\alpha)} \int_{t_{k}}^{t}(t-s)^{\alpha-1} v(s) d s
\end{aligned}
$$

Hence, $u \in F(y)$.

Now, we will prove the existence of a real number $\delta<1$ for which

$$
H_{d}(F(x), F(y)) \leq \delta\|x-y\|_{P C_{*}}, \quad \text { for all } x, y \in P C_{*}([0, T], \mathbb{R})
$$

For each $x, y \in P C_{*}([0, T], \mathbb{R})$ and $h_{1} \in P(x)$, we can find $v_{1}(t) \in F(t, x(t))$ such that, for all $t \in(0, T]$,

$$
h_{1}(t)=\left(t-t_{k}\right)^{\alpha-1} \prod_{i=1}^{k}\left(t_{i}-t_{i-1}\right)^{\alpha-1} c
$$




$$
\begin{aligned}
& +\frac{\left(t-t_{k}\right)^{\alpha-1}}{\Gamma(\alpha)}\left[\int_{t_{k-1}}^{t_{k}}\left(t_{k}-s\right)^{\alpha-1} v_{1}(s) d s\right. \\
& \left.+\sum_{i=1}^{k-1} \prod_{j=1}^{k-i}\left(t_{k-j+1}-t_{k-j}\right)^{\alpha-1} \int_{t_{i-1}}^{t_{i}}\left(t_{i}-s\right)^{\alpha-1} v_{1}(s) d s\right] \\
& +\frac{\left(t-t_{k}\right)^{\alpha-1}}{\Gamma(\alpha)}\left[I_{k}\left(x\left(t_{k}^{-}\right)\right)+\sum_{i=1}^{k-1} \prod_{j=1}^{k-i}\left(t_{k-j+1}-t_{k-j}\right)^{\alpha-1} I_{i}\left(x\left(t_{i}^{-}\right)\right)\right] \\
& +\frac{1}{\Gamma(\alpha)} \int_{t_{k}}^{t}(t-s)^{\alpha-1} v_{1}(s) d s .
\end{aligned}
$$

From $\left(H_{3}\right)$, we have

$$
H_{d}(F(t, x(t)), F(t, y(t))) \leq p(t)|x(t)-y(t)| .
$$

Consequently, exists $w \in F(t, y(t))$ such that

$$
\left|v_{1}(t)-w\right| \leq p(t)|x(t)-y(t)|, \quad t \in(0, T] .
$$

Consider the map $U$ from $(0, T]$ into $\mathcal{P}(\mathbb{R})$ defined by

$$
U(t):=\left\{w(t) \in \mathbb{R}:\left|v_{1}(t)-w\right| \leq p(t)|x(t)-y(t)|\right\} .
$$

From [9, Proposition III. 4], the intersection between $U(t)$ and $F(t, y(t))$ is a measurable set. Therefore, we can find a measurable selection $v_{2}(\cdot)$ for $U(\cdot) \cap F(\cdot, y(\cdot))$. So, $v_{2}(t) \in F(t, y(t))$ and

$$
\left|v_{1}(t)-v_{2}(t)\right| \leq p(t)|x(t)-y(t)|, \quad \text { for all } 0<t \leq T .
$$

For every $0<t \leq T$, we define

$$
\begin{aligned}
h_{1}(t)= & \left(t-t_{k}\right)^{\alpha-1} \prod_{i=1}^{k}\left(t_{i}-t_{i-1}\right)^{\alpha-1} c \\
& +\frac{\left(t-t_{k}\right)^{\alpha-1}}{\Gamma(\alpha)}\left[\int_{t_{k-1}}^{t_{k}}\left(t_{k}-s\right)^{\alpha-1} v_{2}(s) d s\right. \\
& \left.+\sum_{i=1}^{k-1} \prod_{j=1}^{k-i}\left(t_{k-j+1}-t_{k-j}\right)^{\alpha-1} \int_{t_{i-1}}^{t_{i}}\left(t_{i}-s\right)^{\alpha-1} v_{2}(s) d s\right] \\
& +\frac{\left(t-t_{k}\right)^{\alpha-1}}{\Gamma(\alpha)}\left[I_{k}\left(y\left(t_{k}^{-}\right)\right)+\sum_{i=1}^{k-1} \prod_{j=1}^{k-i}\left(t_{k-j+1}-t_{k-j}\right)^{\alpha-1} I_{i}\left(y\left(t_{i}^{-}\right)\right)\right] \\
& +\frac{1}{\Gamma(\alpha)} \int_{t_{k}}^{t}(t-s)^{\alpha-1} v_{2}(s) d s .
\end{aligned}
$$

Thus,

$$
\begin{aligned}
& \left|\left(t-t_{k}\right)^{1-\alpha} h_{1}(t)-\left(t-t_{k}\right)^{1-\alpha} h_{2}(t)\right| \\
\leq & \frac{1}{\Gamma(\alpha)}\left[\int_{t_{k-1}}^{t_{k}}\left(t_{k}-s\right)^{\alpha-1}\left|v_{2}(s)-v_{1}(s)\right| d s\right.
\end{aligned}
$$




$$
\begin{aligned}
& \left.+\sum_{i=1}^{k-1} \prod_{j=1}^{k-i}\left(t_{k-j+1}-t_{k-j}\right)^{\alpha-1} \int_{t_{i-1}}^{t_{i}}\left(t_{i}-s\right)^{\alpha-1}\left|v_{2}(s)-v_{1}(s)\right| d s\right] \\
& +\frac{1}{\Gamma(\alpha)}\left[\left|I_{k}\left(y\left(t_{k}^{-}\right)\right)-I_{k}\left(x\left(t_{k}^{-}\right)\right)\right|+\sum_{i=1}^{k-1} \prod_{j=1}^{k-i}\left(t_{k-j+1}-t_{k-j}\right)^{\alpha-1}\left|I_{i}\left(y\left(t_{i}^{-}\right)\right)-I_{i}\left(x\left(t_{i}^{-}\right)\right)\right|\right] \\
& +\frac{\left(t-t_{k}\right)^{1-\alpha}}{\Gamma(\alpha)} \int_{t_{k}}^{t}(t-s)^{\alpha-1}\left|v_{2}(s)-v_{1}(s)\right| d s .
\end{aligned}
$$

Hence,

$$
\left\|h_{1}-h_{2}\right\|_{P C_{*}} \leq\left[\frac{T^{\alpha}\|p\|_{\infty} \Gamma(\alpha)\left(1+m T^{\alpha-1}\right)}{\Gamma(2 \alpha)}+\frac{m T_{0}^{\alpha-1} L}{\Gamma(\alpha)}\right]\|x-y\|_{P C_{*}},
$$

where $T_{0}=\min _{i=1, \ldots, m}\left(t_{i+1}-t_{i}\right)$. Interchange $x$ by $y$ in the previous computation, we obtain

$$
H_{d}(P(x), P(y)) \leq \delta\|x-y\|_{P C_{*}},
$$

where $\delta=\frac{T^{\alpha}\|p\|_{\infty} \Gamma(\alpha)\left(1+m T^{\alpha-1}\right)}{\Gamma(2 \alpha)}+\frac{m T_{0}^{\alpha-1} L}{\Gamma(\alpha)}<1$. Hence, $P$ is a contraction, and from Lemma 3.4, it has a fixed point $y$ considered as a solution of (1.1).

Step 2. Structure of the solutions set. Let

$$
S_{F}(c)=\left\{y \in P C_{*}([0, T], \mathbb{R}): y \text { is solution of }(1.1)\right\} .
$$

We will prove that $S_{F}(c)$ is compact in $P C_{*}([0, T], \mathbb{R})$. Let $\left\{y_{n}\right\}_{n \in \mathbb{N}} \in S_{F}(c)$, then there exists $v_{n} \in S_{F, y_{n}}$ and $t \in J$ such that

$$
\begin{aligned}
y_{n}(t)= & \left(t-t_{k}\right)^{\alpha-1} \prod_{i=1}^{k}\left(t_{i}-t_{i-1}\right)^{\alpha-1} c \\
& +\frac{\left(t-t_{k}\right)^{\alpha-1}}{\Gamma(\alpha)}\left[\int_{t_{k-1}}^{t_{k}}\left(t_{k}-s\right)^{\alpha-1} v_{n}(s) d s\right. \\
& \left.+\sum_{i=1}^{k-1} \prod_{j=1}^{k-i}\left(t_{k-j+1}-t_{k-j}\right)^{\alpha-1} \int_{t_{i-1}}^{t_{i}}\left(t_{i}-s\right)^{\alpha-1} v_{n}(s) d s\right] \\
& +\frac{\left(t-t_{k}\right)^{\alpha-1}}{\Gamma(\alpha)}\left[I_{k}\left(y\left(t_{k}^{-}\right)\right)+\sum_{i=1}^{k-1} \prod_{j=1}^{k-i}\left(t_{k-j+1}-t_{k-j}\right)^{\alpha-1} I_{i}\left(y\left(t_{i}^{-}\right)\right)\right] \\
& +\frac{1}{\Gamma(\alpha)} \int_{t_{k}}^{t}(t-s)^{\alpha-1} v_{n}(s) d s
\end{aligned}
$$

From $\left(H_{1}\right)$ and $\left(H_{2}\right)$, there exists $M_{1}>0$ such that $\left.\left\|y_{n}\right\|\right|_{P C_{*}} \leq M_{1}$ for every $n \geq 1$, and the set $\left\{y_{1}, y_{2}, \ldots, y_{n}, \ldots\right\}$ is equicontinuous in $P C_{*}([0, T], \mathbb{R})$. By using Theorem 3.1 , we can find a subsequence of $\left(y_{n}\right)$ (still denoted $\left(y_{n}\right)$ ) converges to $y$ in the space $P C_{*}([0, T], \mathbb{R}$. Now, we will prove the existence of $v(\cdot) \in F(\cdot, y(\cdot))$ and an element 
$t \in J$ such that

$$
\begin{aligned}
y(t)= & \left(t-t_{k}\right)^{\alpha-1} \prod_{i=1}^{k}\left(t_{i}-t_{i-1}\right)^{\alpha-1} c \\
& +\frac{\left(t-t_{k}\right)^{\alpha-1}}{\Gamma(\alpha)}\left[\int_{t_{k-1}}^{t_{k}}\left(t_{k}-s\right)^{\alpha-1} v(s) d s\right. \\
& \left.+\sum_{i=1}^{k-1} \prod_{j=1}^{k-i}\left(t_{k-j+1}-t_{k-j}\right)^{\alpha-1} \int_{t_{i-1}}^{t_{i}}\left(t_{i}-s\right)^{\alpha-1} v(s) d s\right] \\
& +\frac{\left(t-t_{k}\right)^{\alpha-1}}{\Gamma(\alpha)}\left[I_{k}\left(y\left(t_{k}^{-}\right)\right)+\sum_{i=1}^{k-1} \prod_{j=1}^{k-i}\left(t_{k-j+1}-t_{k-j}\right)^{\alpha-1} I_{i}\left(y\left(t_{i}^{-}\right)\right)\right] \\
& +\frac{1}{\Gamma(\alpha)} \int_{t_{k}}^{t}(t-s)^{\alpha-1} v(s) d s .
\end{aligned}
$$

Use the fact that $F(\cdot, \cdot)$ is upper semi-continuous, we can show that for every positive real number $\varepsilon$, there exists a positive integer $n_{0}$ such that

$$
v_{n}(t) \in F\left(t, y_{n}(t)\right) \subset F\left(t, y_{n}(t)\right)+\varepsilon B(0,1) \text { a.e. } t \in J \text {, for every } n \geq n_{0} .
$$

Using the compactness of $F(\cdot, \cdot)$ we get the subsequence $v_{n m}(\cdot)$ such that

$$
v_{n m}(\cdot) \rightarrow v(\cdot) \text { and } \quad v(t) \in F(t, y(t)) \text { a.e. } t \in J .
$$

From $\left(H_{1}\right)$, we have

$$
v_{n m}(\cdot) \leq \bar{a} t^{\alpha-1} M_{1}+\bar{b} \quad \text { a.e. } t \in J .
$$

Using Lebesgue's dominated convergence theorem, we obtain that $v \in L^{1}(J, \mathbb{R})$, so $v \in S_{F, y}$. Therefore, for all $t \in J$

$$
\begin{aligned}
y(t)= & \left(t-t_{k}\right)^{\alpha-1} \prod_{i=1}^{k}\left(t_{i}-t_{i-1}\right)^{\alpha-1} c \\
& +\frac{\left(t-t_{k}\right)^{\alpha-1}}{\Gamma(\alpha)}\left[\int_{t_{k-1}}^{t_{k}}\left(t_{k}-s\right)^{\alpha-1} v(s) d s\right. \\
& \left.+\sum_{i=1}^{k-1} \prod_{j=1}^{k-i}\left(t_{k-j+1}-t_{k-j}\right)^{\alpha-1} \int_{t_{i-1}}^{t_{i}}\left(t_{i}-s\right)^{\alpha-1} v(s) d s\right] \\
& +\frac{\left(t-t_{k}\right)^{\alpha-1}}{\Gamma(\alpha)}\left[I_{k}\left(y\left(t_{k}^{-}\right)\right)+\sum_{i=1}^{k-1} \prod_{j=1}^{k-i}\left(t_{k-j+1}-t_{k-j}\right)^{\alpha-1} I_{i}\left(y\left(t_{i}^{-}\right)\right)\right] \\
& +\frac{1}{\Gamma(\alpha)} \int_{t_{k}}^{t}(t-s)^{\alpha-1} v(s) d s .
\end{aligned}
$$

Then $S_{F}(c)$ is compact. 
Let $f \in F$ be a function which is mLL. Consider the following single-valued problem

$$
\left\{\begin{array}{l}
{ }^{R L} D^{\alpha} y(t)=f(t, y(t)) \quad \text { a.e. } t \in J=(0, T], t \neq t_{k}, \\
\lim _{t \rightarrow 0^{+}} t^{1-\alpha} y(t)=c \\
\left.\Delta^{*} y\right|_{t_{k}}=I_{k}\left(y\left(t_{k}^{-}\right)\right) .
\end{array}\right.
$$

Using Banach fixed point theorem, we can prove easily that the problem (3.5) has a unique solution $\bar{x}$. Consider the homotopy $h: S_{F}(c) \times[0,1] \rightarrow S_{F}(c)$ defined by

$$
h(y, \lambda):= \begin{cases}y, & \text { for } \lambda=1 \text { and } y \in S_{F}(c), \\ \bar{x}, & \text { for } \lambda=0\end{cases}
$$

Note that

$$
h(y, \lambda)(t)= \begin{cases}y(t), & \text { for } 0<t \leq \lambda T \\ \bar{x}(t), & \text { for } \lambda T<t \leq T .\end{cases}
$$

We will show that $h$ is a continuous homotopy. Let $\left(y_{n}, \lambda_{n}\right) \in S_{F}(c) \times[0,1]$ such that $\left(y_{n}, \lambda_{n}\right) \rightarrow(y, \lambda)$. We shall show that $h\left(y_{n}, \lambda_{n}\right) \rightarrow h(y, \lambda)$. We have

$$
h\left(y_{n}, \lambda_{n}\right)(t)= \begin{cases}y_{n}(t), & \text { for } t \in\left(0, \lambda_{n} T\right], \\ \bar{x}(t), & \text { for }\left(\lambda_{n} T, T\right] .\end{cases}
$$

(a) If $\lim _{n \rightarrow \infty} \lambda_{n}=0$, then $H(y, 0)(t)=\bar{x}(t)$, for $t \in(0, T]$, hence

$$
\left\|H\left(y_{n}, \lambda_{n}\right)-H(y, \lambda)\right\|_{P C_{*}} \leq\left\|y_{n}-y\right\|_{P C_{*}}+\left\|y_{n}-\bar{x}\right\|_{\left[0, \lambda_{n} T\right]},
$$

which tends to 0 as $n \rightarrow+\infty$. The case $\lim _{n \rightarrow \infty} \lambda_{n}=1$ can be processed in the same way.

(b) If $\lambda_{n} \neq 0$ and $0<\lim _{n \longrightarrow \infty} \lambda_{n}=\lambda<1$. We distinguish two sub-cases.

(i) Since $y_{n} \in S_{F}(c)$, there exists $v_{n} \in S_{F, y_{n}}$ such that for $t \in\left(0, \lambda_{n} T\right]$

$$
\begin{aligned}
y_{n}(t)= & \left(t-t_{k}\right)^{\alpha-1} \prod_{i=1}^{k}\left(t_{i}-t_{i-1}\right)^{\alpha-1} c \\
& +\frac{\left(t-t_{k}\right)^{\alpha-1}}{\Gamma(\alpha)}\left[\int_{t_{k-1}}^{t_{k}}\left(t_{k}-s\right)^{\alpha-1} v_{n}(s) d s\right. \\
& \left.+\sum_{i=1}^{k-1} \prod_{j=1}^{k-i}\left(t_{k-j+1}-t_{k-j}\right)^{\alpha-1} \int_{t_{i-1}}^{t_{i}}\left(t_{i}-s\right)^{\alpha-1} v_{n}(s) d s\right] \\
& +\frac{\left(t-t_{k}\right)^{\alpha-1}}{\Gamma(\alpha)}\left[I_{k}\left(y_{n}\left(t_{k}^{-}\right)\right)+\sum_{i=1}^{k-1} \prod_{j=1}^{k-i}\left(t_{k-j+1}-t_{k-j}\right)^{\alpha-1} I_{i}\left(y_{n}\left(t_{i}^{-}\right)\right)\right] \\
& +\frac{1}{\Gamma(\alpha)} \int_{t_{k}}^{t}(t-s)^{\alpha-1} v_{n}(s) d s .
\end{aligned}
$$

Since $y_{n} \rightarrow y$ as $n \rightarrow \infty$, there exists a positive real number $r>0$ such that $\left\|y_{n}\right\|_{P C_{*}} \leq r$, and as the function $F(\cdot, \cdot)$ is upper semi-continuous, for every 
positive real number $\varepsilon$, there exists a positive integer $n_{0}$ such that for every $n \geq n_{0}$, we have

$$
v_{n}(t) \in F\left(t, y_{n}(t)\right) \subset F\left(t, y_{n}(t)\right)+\varepsilon B(0,1) \quad \text { a.e. } t \in J .
$$

Using the fact that $F(\cdot, \cdot)$ has compact values, we can obtain the subsequence $v_{n m}(\cdot)$ such that

$$
v_{n m}(\cdot) \rightarrow v(\cdot) \quad \text { and } \quad v(t) \in F(t, y(t)) \text { a.e. } t \in J .
$$

From $\left(H_{1}\right)$, we have

$$
v_{n m}(\cdot) \leq \bar{a} t^{\alpha-1} M_{1}+\bar{b} \quad \text { a.e. } t \in J .
$$

Using Lebesgue's dominated convergence theorem, we get $v \in L^{1}(J, \mathbb{R})$, so $v \in S_{F, y}$. Since $I_{k}$ are continuous functions, then, for all $t \in J$, we get

$$
\begin{aligned}
y(t)= & \left(t-t_{k}\right)^{\alpha-1} \prod_{i=1}^{k}\left(t_{i}-t_{i-1}\right)^{\alpha-1} c \\
& +\frac{\left(t-t_{k}\right)^{\alpha-1}}{\Gamma(\alpha)}\left[\int_{t_{k-1}}^{t_{k}}\left(t_{k}-s\right)^{\alpha-1} v(s) d s\right. \\
& \left.+\sum_{i=1}^{k-1} \prod_{j=1}^{k-i}\left(t_{k-j+1}-t_{k-j}\right)^{\alpha-1} \int_{t_{i-1}}^{t_{i}}\left(t_{i}-s\right)^{\alpha-1} v(s) d s\right] \\
& +\frac{\left(t-t_{k}\right)^{\alpha-1}}{\Gamma(\alpha)}\left[I_{k}\left(y\left(t_{k}^{-}\right)\right)+\sum_{i=1}^{k-1} \prod_{j=1}^{k-i}\left(t_{k-j+1}-t_{k-j}\right)^{\alpha-1} I_{i}\left(y\left(t_{i}^{-}\right)\right)\right] \\
& +\frac{1}{\Gamma(\alpha)} \int_{t_{k}}^{t}(t-s)^{\alpha-1} v(s) d s .
\end{aligned}
$$

(ii) Since $t \in\left(\lambda_{n} T, T\right]$, then

$$
h\left(y_{n}, \lambda_{n}\right)(t)=h(y, \lambda)(t)=\bar{x} .
$$

Thus,

$$
\left\|h\left(y_{n}, \lambda_{n}\right)-h(y, \lambda)\right\|_{P C_{*}} \rightarrow 0 \quad n \rightarrow \infty .
$$

Consequently, $h$ is continuous, and hence, $S_{F}(c)$ is contractible to the point $\bar{x}$. Therefore, $S_{F}(c)$ is an acyclic space.

Example 3.1 (An application). Consider

$$
\left\{\begin{array}{l}
R L D^{\frac{1}{2}} y(t) \in F(t, y(t)) \quad \text { a.e. } t \in(0,1], t \neq \frac{1}{2}, \\
\lim _{t \rightarrow 0^{+}} t^{\frac{1}{2}} y(t)=\frac{1}{4}, \\
\left.\Delta^{*} y\right|_{t=\frac{1}{2}}=\frac{1}{9}\left|\sin \left(y\left(\frac{1}{2}^{-}\right)\right)\right|+1,
\end{array}\right.
$$


where $T=1, m=1, t_{1}=\frac{1}{2}$,

$$
F(t, x)=\left[0, \frac{1}{9} \sin x+\frac{|x|}{t+9}+\frac{1}{9}\right]
$$

and

Clearly,

$$
I_{1}(u)=\frac{1}{9}|\sin (u)|+1, \quad \text { for } u \in \mathbb{R}
$$

$$
\begin{aligned}
\sup _{v \in F(t, x)}|v| & \leq \frac{1}{9}+\frac{1}{9}|\sin x|+\frac{|x|}{t+9}, \\
H_{d}(F(t, x), F(t, y)) & \leq\left[\frac{1}{t+9}+\frac{1}{9}\right]|x-y|
\end{aligned}
$$

and

$$
\left|I_{1}(u)\right| \leq \frac{1}{9}|\sin (u)|+1, \quad\left|I_{1}(u)-I_{1}(v)\right| \leq \frac{1}{9}|u-v| .
$$

Let $p(t)=\frac{1}{9}+\frac{1}{t+9}$. Then $\|p\|_{\infty}=\frac{2}{9}$ and

$$
\frac{T^{\alpha}\|p\|_{\infty} \Gamma(\alpha)\left(1+m T^{\alpha-1}\right)}{\Gamma(2 \alpha)}+\frac{m T_{0}^{\alpha-1} L}{\Gamma(\alpha)} \simeq 0,73965<1 .
$$

Theorem 3.2 confirms that (3.6) has at least one solution. In addition, it is clear that $F$ is a mLL-selectionable multivalued map (i.e., the function $f(t, u)=\frac{1}{9} \sin u+\frac{|u|}{t+9}+\frac{1}{9}$ is measurable, locally-Lipchitzian) with compact and convex values. Consequently, the solution set is contractible and compact, and hence it is acyclic.

Acknowledgements. I would like to thank the editor and referees for his/her suggestions that improved the quality of the paper. Also I thank my PhD supervisor Pr. Hedia Benaouda for his helpful comments and encouragement.

\section{REFERENCES}

[1] S. Abbes and M. Benchohra, Advanced Functional Evolution Equations and Inclusions, Springer, New York, London, 2015.

[2] R. P. Agarwal, M. Benchohra and S. Hamani, A survey on existence results for boundary value problems of nonlinear fractional differential equations and inclusions, Acta Appl. Math. 109 (2010), 973-1033.

[3] R. P. Agarwal, M. Benchohra, J. Nieto and A. Ouahab, Some results for integral inclusions of Volterra type in Banach spaces, Adv. Difference Equ. (2010), DOI 10.1155/2010/798067.

[4] Z. Agur, L. Cojocaru, G. Mazaur, R. M. Anderson and Y. L. Danon, Pulse mass measles vaccination across age cohorts, Proc. Nat. Acad. Sci. USA 90 (1993), 11698-11702.

[5] J. Andres and L. Górniewicz, Topological Principles for Boundary Value Problems, Kluwer, Dordrecht, 2003.

[6] J. P. Aubin and A. Cellina, Differential Inclusions, Springer-Verlag, New York, 1984.

[7] J. P. Aubin and H. Frankowska, Set-Valued Analysis, Birkhauser, Boston, 1990. 
[8] M. Benchohra, J. Nieto and A. Ouahab, Impulsive differential inclusions involving evolution operators in separable Banach spaces, Ukrainian Math. J. 64 (2012), 991-1018.

[9] C. Castaing and M. Valadier, Convex Analysis and Measurable Multifunctions, Lecture Notes in Mathematics 580, Springer-Verlag, Berlin, Heidelberg, New York, 1977.

[10] Y. Chalco-Cano, J. J. Nieto, A. Ouahab and H. Román-Flores, Solution set for fractional differential equations with Riemann-Liouville derivative, Fract. Calc. Appl. Anal. 16 (2013), 682-694.

[11] H. Covitz and S. B. Nadler, Multivalued contraction mappings in generalized metric spaces, Israel J. Math. 8 (1970), 5-11.

[12] K. Deimling, Multivalued Differential Equations, De Gruyter, Berlin, New York, 1992.

[13] D. Delbosco and L. Rodino, Existence and uniqueness for a nonlinear fractional differential equation, J. Math. Anal. Appl. (1996), 609-625.

[14] K. Djebali, L. Górniewicz and A. Ouahab, Solutions Sets for Differential Equations and Inclusions, De Gruyter Series in Nonlinear Analysis and Applications 18, de Gruyter, Berlin, 2013.

[15] S. Djebali, L. Górniewicz and A. Ouahab, Existence and Structure of Solution Sets for Impulsive Differential Inclusions, a Survey, Lecture Notes in Nonlinear Analysis 13, Nicolaus Copernicus University, Juliusz Schauder Center for Nonlinear Studies, Torun, 2012.

[16] S. Djebali, L. Górniewicz and A. Ouahab, Topological structure of solution sets for impulsive differential inclusions in Fréchet spaces, Nonlinear Anal. 74 (2011), 2141-2169.

[17] R. Dragoni, P. Nistri, P. Zeccaand and J. W. Macki, Solution Sets of Differential Equations in Abstract Spaces, Longman, Edinburgh, 1996.

[18] A. M. A. El-Sayed, Nonlinear functional differential equations of arbitrary orders, Nonlinear Anal. 33 (1998), 181-186.

[19] A. M. A. El-Sayed and A. G. Ibrahim, Multivalued fractional differential equations, Appl. Math. Comput. 68 (1995), 15-25.

[20] L. Gaul, P. Klein and S. Kempfle, Damping description involving fractional operators, Mechanical Systems and Signal Processing 5 (1991), 81-88.

[21] W. G. Glockle and T. F. Nonnenmacher, A fractional calculus approach of self-similar protein dynamics, Biophysical Journal 68 (1995), 46-53.

[22] L. Górniewicz, On the solution sets of differential inclusions, J. Math. Anal. Appl. (1986), 235-244.

[23] L. Górniewicz, Topological Fixed Point Theory of Multivalued Mappings, Mathematics and its Applications 495, Kluwer Academic Publishers, Dordrecht, 1999.

[24] A. Granas and J. Dugundji, Fixed Point Theory, Springer-Verlag, New York, 2003.

[25] A. Grudzka and S. Ruszkowski, Structure of the solutions set to differential inclusions with impulses and variable times, Electronic J. Diff. Equ. (2015), 1-16.

[26] J. Henderson and A. Ouahab, Impulsive differential inclusions with fractional order, Comput. Math. Appl. 59 (2010), 1191-1226.

[27] S. Hu and N. S. Papageorgiou, Handbook of Multi-Valued Analysis, Volume I: Theory, Kluwer, Dordrecht, 1997.

[28] S. Hu and N. S. Papageorgiou, Handbook of Multi-Valued Analysis Volume II: Applications, Kluwer, Dordrecht, 2000.

[29] M. Kamenskii, V. Obukhovskii and P. Zecca, Condensing Multivalued Maps and Semilinear Differential Inclusions in Banach Spaces, Nonlinear Analysis and Applications 7, Walter de Gruyter, Berlin, New York, 2001.

[30] E. Krüger-Thiemer, Formal theory of drug dosage regimens. I, J. Theoret. Biol. 13 (1966), 212-235.

[31] E. Krüger-Thiemer, Formal theory of drug dosage regimens: II. The exact plateau effect, J. Theoret. Biol. 23 (1969), 169-190. 
[32] A. Lasota and Z. Opial, An application of the Kakutaniky fan theorem in the theory of ordinary differential equations, Bull. Acad. Pol. Sci. Ser. Sci. Math. Astronom. Phys. 13 (1965), 781-786.

[33] J. M. Lasry and R. Robert, Analyse Non Lineaire Multivoque, Centre de Recherche de Mathématique de la Décision, Université de Dauphine, Paris, CNRS, 1976.

[34] F. Metzler, W. Schick, H. G. Kilian and T. F. Nonnenmacher, Relaxation in filled polymers, a fractional calculus approach, The Journal of Chemical Physics (1995), 7180-7186.

[35] J. Musielak, Introduction to Functional Analysis, PWN, Warsaw, 1976.

[36] D. Wagner, Survey of measurable selection theorems, SIAM J. Control Optim. 15 (1977), 859903.

[37] Z. Yong, W. Rong-Nian and P. Li, Topological Structure of the Solution Set for Evolution Inclusions, Springer-Verlag, New York, 2017.

[38] S. Zhang, Positive solutions for boundary-value problems of nonlinear fractional diffrential equations, Electron. J. Differential Equations 36 (2006), 1-12.

[39] Y. Zhou, Basic Theory of Fractional Differential Equations, World Scientific, Singapore, 2014.

[40] Y. Zhou, Fractional Evolution Equations and Inclusions, Analysis and Control, Elsevier, Amsterdam, 2015.

${ }^{1}$ Department of Mathematics,

Djillali Liabes University of Sidi Bel-AbBès,

P.O. Box 89, Sidi Bel-AbBès 22000 , Algeria

Email address: beddani20040yahoo.fr 
Kragujevac Journal of Mathematics

Volume 46(1) (2022), Pages 65-84.

\title{
UNIFORMLY CONVERGENT NUMERICAL METHOD FOR SINGULARLY PERTURBED DELAY PARABOLIC DIFFERENTIAL EQUATIONS ARISING IN COMPUTATIONAL NEUROSCIENCE
}

\author{
MESFIN MEKURIA WOLDAREGAY ${ }^{1}$ AND GEMECHIS FILE DURESSA ${ }^{1}$
}

\begin{abstract}
The motive of this work is to develop $\varepsilon$-uniform numerical method for solving singularly perturbed parabolic delay differential equation with small delay. To approximate the term with the delay, Taylor series expansion is used. The resulting singularly perturbed parabolic differential equation is solved by using non-standard finite difference method in spatial direction and implicit Runge-Kutta method for the resulting system of IVPs in temporal direction. Theoretically the developed method is shown to be accurate of order $O\left(N^{-1}+(\Delta t)^{2}\right)$ by preserving $\varepsilon$-uniform convergence. Two numerical examples are considered to investigate $\varepsilon$ uniform convergence of the proposed scheme and the result obtained agreed with the theoretical one.
\end{abstract}

\section{INTRODUCTION}

The class of differential difference equations which have characteristics of delay and singularly perturbed behavior is known as singularly perturbed differential difference equations or singularly perturbed delay differential equations (SPDDEs). SPDDEs plays an important role in modeling real life phenomena in Bioscience, Control Theory, Economics and Engineering [6]. A few application areas are the mathematical modeling of: epidemiology and population dynamics [11], physiological kinetics [1], production of blood cell [16]. Singularly perturbed differential equations relate an unknown function to its derivatives evaluated at the same instance. Whereas, singularly perturbed delay differential equations model physical problems for which the evaluation depend on the present state of the system and also on the past history. In

Key words and phrases. Delay differential equation, method of line, non-standard finite difference, singular perturbation.

2010 Mathematics Subject Classification. Primary: 65M06, 65M20. Secondary: 65N06, 65N12.

DOI 10.46793/KgJMat2201.065W

Received: June 11, 2019.

Accepted: August 29, 2019. 
general, when the perturbation parameter approaches to zero the smoothness of the solution of the SPDDEs deteriorates and it forms a boundary layer(s) [19]. The time dependent problem i.e. singularly perturbed parabolic delay differential equations (SPPDDEs) have applications in the study of modeling of neuronal variability [23].

Various mathematical models are given in books $[25,26]$, for the determination of the behavior of a neuron to random synaptic inputs. In 1991, Musila and Lansky [18] gives generalization for the Stein's model [23] and proposed a model to treat the time evolution trajectories of the membrane potential in terms of SPPDDEs as

$$
\frac{\partial u}{\partial t}=\frac{\sigma^{2}}{2} \frac{\partial^{2} u}{\partial x^{2}}+\left(\mu_{D}-\frac{x}{\tau}\right) \frac{\partial u}{\partial x}+\lambda_{s} u\left(x+a_{s}, t\right)+\omega_{s} u\left(x+i_{s}, t\right)-\left(\lambda_{s}+\omega_{s}\right) u(x, t),
$$

where $\mu_{D}$ and $\sigma$ are diffusion moments of Wiener process characterizing the influence of dendritic synapses on the cell excitability. The excitatory input contributes to the membrane potential by an amplitude $a_{s}$ with intensity $\lambda_{s}$ and similarly the inhibitory input contributes by an amplitude $i_{s}$ with intensity $\omega_{s}$. The first derivative term is because of the exponential decay between two consecutive jumps caused by the input processes. The membrane potential decays exponentially to the resting level with a membrane time constant $\tau$. This model makes available time evolution of the trajectories of the membrane potential. The model in (1.1) is a singularly perturbed parabolic differential difference equation, one can hardly derive its exact solution. Hence, to simulate this model, one has to land to a very suitable numerical methods. In the last few years, scholar's have devoted for the development of numerical solution of this problem. In addition to that, authors try to show the influence of the delay parameter on the behavior of the solution. In research articles $[2-4,12,13,19,20]$ authors develop different numerical schemes to study a classes of SPPDDEs and discussed the effect of shifts on the solution.

In this paper, we construct and analyze a non-standard finite difference scheme which utilizes on uniform mesh. The proposed scheme uses the procedures of method of line, which consist of non-standard finite difference operator for the spatial discretization and Runge-Kutta method for the time discretization. For the theoretical analysis, the global error is decomposed into two parts: the first is due to the spatial discretization and the second is due to the temporal discretization of the semi-discrete problem obtained after the spatial discretization.

The main contribution of this work is, to develop $\varepsilon$-uniform numerical scheme for the SPPDDEs containing small delay on the spatial variable. In the proposed method, it is not required to have any restriction on the mesh generation.

This paper is organized as follows. In Section 1 a brief introduction about the problem is given, in Section 2 definition of the problem and the behavior of its analytical solution is given. In Section 3, discretizing the spatial domain and techniques of non-standard finite difference is discussed, and the $\varepsilon$-uniform convergence of the semidiscrete problem is proved. Next, Runge-Kutta method used for the system of IVPs resulted from spatial discretization and discuss the convergence of the discrete scheme. 
In Section 4, numerical examples and results are given to validate the theoretical analysis and finally, in Section 5, the conclusion of the work done is presented.

Notations. Through out this paper $N, M$ denoted for the number of mesh elements in space and time direction respectively. $C$ (in some case indexed) is denoted for positive constant independent of perturbation parameter and $N$. The norm $\|\cdot\|_{\Omega_{x}^{N} \times \Omega_{t}^{M}}$ is used to denote discrete maximum norm.

\section{Problem Formulation}

A class of singularly perturbed parabolic differential difference equation with delay on the spatial variable on domain $D=\Omega_{x} \times \Omega_{t}$ is given by

$$
\left\{\begin{array}{l}
\frac{\partial u}{\partial t}-\varepsilon^{2} \frac{\partial^{2} u}{\partial x^{2}}+a(x) \frac{\partial u}{\partial x}+\alpha(x) u(x-\delta, t)+\beta(x) u(x, t)=f(x, t), \\
u(x, 0)=u_{0}(x), x \in D_{0}=\left\{(x, 0): x \in \bar{\Omega}_{x}\right\} \\
u(x, t)=\phi(x, t),(x, t) \in D_{L}=\left\{(x, t):-\delta \leq x \leq 0, t \in \Omega_{t}\right\} \\
u(1, t)=\psi(t), \quad t \in \Omega_{t}
\end{array}\right.
$$

where $(x, t) \in D=\Omega_{x} \times \Omega_{t}=(0,1) \times(0, T]$ for fixed positive number $\mathrm{T}, \varepsilon$ is a singular perturbation parameter with $0<\varepsilon \ll 1$ and $\delta$ is delay parameter assumed to be sufficiently small as order of $o(\varepsilon)$. We assume, the functions $a(x), \alpha(x), \beta(x)$ and $f(x, t), u_{0}(x), \phi(x, t)$ and $\psi(x, t)$ are sufficiently smooth, bounded and independent of $\varepsilon$. The coefficients of reaction term $\beta(x)$ and delay term $\alpha(x)$ are assumed to satisfy $\alpha(x)+\beta(x) \geq \theta>0$, for all $x \in \bar{\Omega}_{x}$, for some positive constant $\theta$. This condition ensure that the solution of (2.1) exhibits boundary layer in the neighborhood of $D_{L}=\left\{(x, t):-\delta \leq x \leq 0, t \in \Omega_{t}\right\}$ if $a(x)-\delta \beta(x)<0$ or in the neighborhood of $D_{R}=\left\{(x, t): x=1, t \in \Omega_{t}\right\}$ if $a(x)-\delta \beta(x)>0$ for $x \in \bar{\Omega}_{x}$.

When the shift parameter is zero (i.e., $\delta=0$ ) the above equation reduces to a singularly perturbed parabolic differential equation, which exhibits layer(s) depending on the value of $a(x)$. If $a(x)<0$ a boundary layer appears in the neighborhood of $x=0$, in case $a(x)>0$ corresponds to existence of a boundary layer near $x=1$. For the case $a(x)$ change sign interior layer will appear on the solution of the problem [9]. The layer is maintained for $\delta \neq 0$ but sufficiently small.

When the shift parameter $\delta<\varepsilon$, the use of Taylor's series expansion for the term containing shift is valid [24].

2.1. Estimate for the delay term. From the assumption $\delta<\varepsilon$, by using Taylor series approximation for $u(x-\delta, t)$, we obtain

$$
u(x-\delta, t) \approx u(x, t)-\delta \frac{\partial u}{\partial x}(x, t)+\frac{\delta^{2}}{2} \frac{\partial^{2} u}{\partial x^{2}}(x, t)+O\left(\delta^{3}\right)
$$


Now, substituting this approximations into (2.1), we obtain

$$
\left\{\begin{array}{l}
\frac{\partial u}{\partial t}-c_{\varepsilon}(x) \frac{\partial^{2} u}{\partial x^{2}}+p(x) \frac{\partial u}{\partial x}+q(x) u(x, t)=f(x, t) \\
u(x, 0)=u_{0}(x), \quad x \in \bar{\Omega} \\
u(0, t)=\phi(0, t), \quad t \in \bar{\Omega}_{t} \\
u(1, t)=\psi(1, t), \quad t \in \bar{\Omega}_{t}
\end{array}\right.
$$

where $c_{\varepsilon}(x)=\varepsilon^{2}-\frac{\delta^{2}}{2} \alpha(x), p(x)=a(x)-\delta \alpha(x), q(x)=\alpha(x)+\beta(x)$. For small $\delta$, equations in (2.1) and (2.3) are asymptotically equivalent, because the difference between the two equations is order of $O\left(\delta^{3}\right)$. Now we assume again $0<c_{\varepsilon}(x) \leq$ $\varepsilon^{2}-\delta^{2} C_{1}=c_{\varepsilon}$, where $\alpha(x) \geq 2 C_{1}$. We also assumed that $p(x)=a(x)-\delta \alpha(x) \geq p^{*}>0$, which guarantee the occurrence of boundary layer in the neighborhood of $x=1$. The other case $p(x)=a(x)-\delta \alpha(x) \leq p^{*}<0$, imply the occurrence of the boundary layer in the neighborhood of $x=0$ and can be treated in similar manner.

We impose the compatibility conditions

$$
\left\{\begin{array}{l}
u_{0}(0)=\phi(0,0) \\
u_{0}(1)=\psi(1,0)
\end{array}\right.
$$

and

$$
\left\{\begin{array}{l}
\frac{\partial \phi(0,0)}{\partial t}-c_{\varepsilon} \frac{\partial^{2} u_{0}(0)}{\partial x^{2}}+p(0) \frac{\partial u_{0}(0)}{\partial x}+q(0) u_{0}(0)=f(0,0), \\
\frac{\partial \psi(1,0)}{\partial t}-c_{\varepsilon} \frac{\partial^{2} u_{0}(1)}{\partial x^{2}}+p(1) \frac{\partial u_{0}(1)}{\partial x}+q(1) u_{0}(1)=f(1,0),
\end{array}\right.
$$

so that the data matches at the two corners $(0,0)$ and $(1,0)$. The considered case is when boundary layer occurs near $x=1$. Now, using compatibility conditions in (2.4) and (2.5), we have the conditions that guarantee the existence of a constant $C$ independent of $c_{\varepsilon}$ such that for all $(x, t) \in \bar{D}$

$$
\begin{aligned}
|u(x, t)-u(x, 0)| & =\left|u(x, t)-u_{0}(x)\right| \leq C t \text { and } \\
|u(x, t)-u(0, t)| & =|u(x, t)-\phi(0, t)| \leq C(1-x),
\end{aligned}
$$

for the detail of this one can see [21, page 105].

Remark 2.1. We note that there does not exist a constant $C$ independent of $c_{\varepsilon}$ such that $|u(x, t)-u(1, t)|=|u(x, t)-\psi(1, t)| \leq C x$, since a regular boundary layer exists near $x=1$.

By setting $c_{\varepsilon}=0$ in (2.3) we obtain the reduced problem as:

$$
\left\{\begin{array}{l}
\frac{\partial u^{0}}{\partial t}+p(x) \frac{\partial u^{0}}{\partial x}+q(x) u^{0}(x, t)=f(x, t), \quad \text { for all }(x, t) \in D \\
u^{0}(x, 0)=u_{0}(x), \quad x \in \bar{\Omega}_{x}, \\
u^{0}(0, t)=\phi_{0}(t), \quad t \in \bar{\Omega}_{t} .
\end{array}\right.
$$

This is a first order hyperbolic PDE with initial data given along the two sides $t=0$ and $x=0$ of the domain $\bar{D}$. For small values of $c_{\varepsilon}$ the solution $u(x, t)$ of the problem 
in $(2.3)$ will be very close to $u^{0}(x, t)$. To obtain error bounds on the solution of the difference scheme, we assume that the solution of the reduced problem in (2.6) is sufficiently smooth.

2.2. Properties of continuous solution. To show a boundedness of the solutions $u(x, t)$ of $(2.3)$, we assume the initial condition to be zero. Since $u_{0}(x)$ is assumed sufficiently smooth and using the property of norm, we prove the following lemma.

Lemma 2.1. The bound on the solution $u(x, t)$ of the continuous problem in (2.3) is given by

$$
|u(x, t)| \leq C, \quad \text { for all }(x, t) \in \bar{D} .
$$

Proof. From the compatibility condition $|u(x, t)-u(x, 0)|=\left|u(x, t)-u_{0}(x)\right| \leq C t$, we have $|u(x, t)|-\left|u_{0}(x)\right| \leq\left|u(x, t)-u_{0}(x)\right| \leq C t$, which implies $|u(x, t)| \leq C t+\left|u_{0}(x)\right|$ for all $(x, t) \in \bar{D}$, since $t \in[0, T]$ and $u_{0}(x)$ is bounded it implies $|u(x, t)| \leq C$.

Lemma 2.2 (Continuous maximum principle). Let $v$ be a sufficiently smooth function defined on $D$ which satisfies $v(x, t) \geq 0$ for all $(x, t) \in \partial D$. Then $L v(x, t)>0$ for all $(x, t) \in D$ implies that $v(x, t) \geq 0$ for all $(x, t) \in \bar{D}$.

Proof. Let $\left(x^{*}, t^{*}\right)$ be such that

$$
v\left(x^{*}, t^{*}\right)=\min _{(x, t) \in \bar{D}} v(x, t)
$$

and suppose that $v\left(x^{*}, t^{*}\right)<0$. It is clear that $v\left(x^{*}, t^{*}\right) \notin \partial D$. So, we have

$$
L v\left(x^{*}, t^{*}\right)=v_{t}\left(x^{*}, t^{*}\right)-c_{\varepsilon} v_{x x}\left(x^{*}, t^{*}\right)+p(x) v_{x}\left(x^{*}, t^{*}\right)+q(x) v\left(x^{*}, t^{*}\right) .
$$

Since

$$
v\left(x^{*}, t^{*}\right)=\min _{(x, t) \in \bar{D}} v(x, t),
$$

which implies $v_{x}\left(x^{*}, t^{*}\right)=0, v_{t}\left(x^{*}, t^{*}\right)=0$ and $v_{x x}\left(x^{*}, t^{*}\right) \geq 0$ and implies that $L v\left(x^{*}, t^{*}\right)<0$, which contradict to the assumption made above. Here, we have $L v\left(x^{*}, t^{*}\right)>0$ for all $(x, t) \in D$. Hence, $v(x, t) \geq 0$ for all $(x, t) \in D$, which complete the proof.

Lemma 2.3 (Stability estimate). Let $u(x, t)$ be the solution of the continuous problem in (2.3). Then we have the bound

$$
u \leq \theta^{-1}\|f\|+\max \left\{u_{0}(x), \max \{\phi(x, t), \psi(x, t)\}\right\},
$$

where $\|f\|=\max |f(x, t)|$.

Proof. We define two barrier functions $\vartheta^{ \pm}$as

$$
\vartheta^{ \pm}(x, t)=\theta^{-1}\|f\|+\max \left\{u_{0}(x), \max \{\phi(x, t), \psi(x, t)\}\right\} \pm u(x, t) .
$$

At the initial value

$$
\vartheta^{ \pm}(x, 0)=\theta^{-1}\|f\|+\max \left\{u_{0}(x), \max \{\phi(x, t), \psi(x, t)\}\right\} \pm u(x, 0) \geq 0 .
$$


At the boundary points

$$
\begin{aligned}
& \vartheta^{ \pm}(0, t)=\theta^{-1}\|f\|+\max \left\{u_{0}(0), \max \{\phi(0, t), \psi(0, t)\}\right\} \pm u(0, t) \geq 0, \\
& \vartheta^{ \pm}(1, t)=\theta^{-1}\|f\|+\max \left\{u_{0}(1), \max \{\phi(1, t), \psi(1, t)\}\right\} \pm u(1, t) \geq 0
\end{aligned}
$$

and

$$
\begin{aligned}
L \vartheta^{ \pm}(x, t)= & \vartheta_{t}^{ \pm}(x, t)-c_{\varepsilon} \vartheta_{x x}^{ \pm}(x, t)+p(x) \vartheta_{x}^{ \pm}(x, t)+q(x) \vartheta^{ \pm}(x, t) \\
= & \left(\max \left\{\phi_{t}(x, t), \psi_{t}(x, t)\right\} \pm u_{t}(x, t)\right)-c_{\varepsilon}\left(\max \left\{\phi_{x x}(x, t), u_{0 x x}(x), \psi_{x x}(x, t)\right\}\right. \\
& \left. \pm u_{x x}(x, t)\right)+p(x)\left(\max \left\{u_{0 x}(x, t), \max \left\{\phi_{x}(x, t), \psi_{x}(x, t)\right\}\right\} \pm u_{x}(x, t)\right) \\
& +q(x)\left(\theta^{-1}\|f\|+\max \left\{u_{0}(x), \max \{\phi(x, t), \psi(x, t)\}\right\} \pm u(x, t)\right) \\
\geq & 0,
\end{aligned}
$$

since $c_{\varepsilon} \geq 0, p(x) \geq p^{*}>0$ and $q(x) \geq \theta>0$. This implies that $L \vartheta^{ \pm}(x, t) \geq 0$. Hence, by maximum principle we have,

$$
\vartheta^{ \pm}(x, t) \geq 0, \quad \text { for all }(x, t) \in \bar{D}
$$

which implies

$$
u(x, t) \leq \theta^{-1}\|f\|+\max \left\{u_{0}(x), \max \{\phi(x, t), \psi(x, t)\}\right\} .
$$

Hence, the proof is completed.

Lemma 2.4. The bound on the derivative of the solution $u(x, t)$ of the problem in (2.3) with respect to $x$ is given by

$$
\left|\frac{\partial^{i} u(x, t)}{\partial x^{i}}\right| \leq C\left(1+c_{\varepsilon}^{-i} \exp \left(-p^{*}(1-x) / c_{\varepsilon}\right)\right), \quad(x, t) \in \bar{D}, i=0,1,2,3,4 .
$$

Proof. See in [10].

\section{Formulation of Numerical Scheme}

3.1. Discretization in spatial direction. The theoretical basis of non-standard discrete modeling method is based on the development of exact finite difference method. In [17], Micken's presented techniques and rules for developing non-standard FDMs for different problem types. In Mickens's rules, to develop a discrete scheme, denominator function for the discrete derivatives must be expressed in terms of more complicated functions of step sizes than those used in the standard procedures. These complicated functions constitutes a general property of the schemes, which is useful while designing reliable schemes for such problems. 
For the problem of the form in (2.3), in order to construct exact finite difference scheme we follow the procedures used in [3]. Consider the constant coefficient subequations given in (3.1) and (3.2) by ignoring the time variable as

$$
\begin{aligned}
-c_{\varepsilon} \frac{d^{2} u(x)}{d x^{2}}+p^{*} \frac{d u(x)}{d x}+\theta u(x) & =0, \\
-c_{\varepsilon} \frac{d^{2} u(x)}{d x^{2}}+p^{*} \frac{d u(x)}{d x} & =0,
\end{aligned}
$$

where $p(x) \geq p^{*}$ and $q(x) \geq \theta$. Thus the problem (3.1) has two independent solutions namely $\exp \left(\lambda_{1} x\right)$ and $\exp \left(\lambda_{2} x\right)$ with

$$
\lambda_{1,2}=\frac{-p^{*} \pm \sqrt{\left(p^{*}\right)^{2}+4 c_{\varepsilon} \theta}}{-2 c_{\varepsilon}} .
$$

We discretize the spatial domain $[0,1]$, using uniform mesh length $\Delta x=h$ such that $\Omega_{x}^{N}=\left\{x_{i}=x_{0}+i h, i=1,2, \ldots, N, x_{0}=0, x_{N}=1, h=1 / N\right\}$, where $\mathrm{N}$ is the number of mesh points in spatial direction. We denote the approximate solution of $u(x)$ at $x_{i}$ 's by $U_{i}$. Now our objective is to calculate a difference equation which has the same general solution as the problem (3.1) has at the grid point $x_{i}$ given by $U_{i}=A_{1} \exp \left(\lambda_{1} x_{i}\right)+A_{2} \exp \left(\lambda_{2} x_{i}\right)$ Using the procedures used in [17], we have

$$
\operatorname{det}\left[\begin{array}{ccc}
U_{i-1} & \exp \left(\lambda_{1} x_{i-1}\right) & \exp \left(\lambda_{2} x_{i-1}\right) \\
U_{i} & \exp \left(\lambda_{1} x_{i}\right) & \exp \left(\lambda_{2} x_{i}\right) \\
U_{i+1} & \exp \left(\lambda_{1} x_{i+1}\right) & \exp \left(\lambda_{2} x_{i+1}\right)
\end{array}\right]=0
$$

Simplifying (3.4), we obtain that

$$
\exp \left(\frac{p^{*} h}{2 c_{\varepsilon}}\right) U_{i-1}-2 \cosh \left(\frac{h \sqrt{\left(p^{*}\right)^{2}+4 c_{\varepsilon} \theta}}{2 c_{\varepsilon}}\right) U_{i}+\exp \left(-\frac{p^{*} h}{2 c_{\varepsilon}}\right) U_{i+1}=0
$$

is an exact difference scheme for (3.1).

After doing the arithmetic manipulation and rearrangement on (3.5) we obtain

$$
-c_{\varepsilon} \frac{U_{i-1}-2 U_{i}+U_{i+1}}{\frac{h c_{\varepsilon}}{p^{*}}\left(\exp \left(\frac{p^{*} h}{c_{\varepsilon}}\right)-1\right)}+p^{*} \frac{U_{i}-U_{i-1}}{h}=0 .
$$

The denominator function becomes $\gamma^{2}=\frac{h c_{\varepsilon}}{p^{*}}\left(\exp \left(\frac{h p^{*}}{c_{\varepsilon}}\right)-1\right)$. Adopting this denominator function for the variable coefficient problem, we write it as

$$
\gamma_{i}^{2}=\frac{h c_{\varepsilon}}{p\left(x_{i}\right)}\left(\exp \left(\frac{h p\left(x_{i}\right)}{c_{\varepsilon}}\right)-1\right)
$$

where $\gamma_{i}^{2}$ is a function of $c_{\varepsilon}, p_{i}$ and $h$, where $p_{i}$ is denoted for $p\left(x_{i}\right)$. By using (3.7) in to the main semi-descrete scheme, we obtain

$$
\frac{d U_{i}(t)}{d t}-c_{\varepsilon} \frac{U_{i+1}(t)-2 U_{i}(t)+U_{i-1}(t)}{\gamma_{i}^{2}}+p\left(x_{i}\right) \frac{U_{i}(t)-U_{i-1}(t)}{h}+q\left(x_{i}\right) U_{i}(t)=f\left(x_{i}, t\right) .
$$


Let $U_{i}(t)$ is denoted for the approximation of $u\left(x_{i}, t\right)$. By using the non-standard finite difference approximation. At this stage the problem in (2.3) reduces to semi-discrete form as

$$
\left\{\begin{aligned}
L^{h} U_{i}(t) \equiv & \frac{d U_{i}(t)}{d t}-c_{\varepsilon} \frac{U_{i+1}(t)-2 U_{i}(t)+U_{i-1}(t)}{\gamma_{i}^{2}}+p\left(x_{i}\right) \frac{U_{i}(t)-U_{i-1}(t)}{h} \\
& +q\left(x_{i}\right) U_{i}(t)=f_{i}(t), \\
U_{0}(t)= & \phi(0, t), \\
U_{N}(t)= & \psi(1, t) \quad \text { and } \\
U_{i}(0)= & u_{0}\left(x_{i}\right) .
\end{aligned}\right.
$$

The system of IVPs in (3.8) can be written in compact form as

$$
\frac{d U_{i}(t)}{d t}=A U_{i}(t)+F_{i}(t)
$$

where $A$ is a tridiagonal matrix of size $N-1 \times N-1$ and $U_{i}(t)$ and $F_{i}(t)$ are column vectors with $N-1$ entries. The entries of $A$ and $F$ are given respectively as

$$
\left\{\begin{array}{l}
a_{i i}=\frac{2 c_{\varepsilon}}{\gamma_{i}^{2}}+\frac{p\left(x_{i}\right)}{h}+q\left(x_{i}\right), \quad i=1,2, \ldots, N-1, \\
a_{i i+1}=-\frac{c_{\varepsilon}}{\gamma_{i}^{2}}, \quad i=1,2, \ldots, N-2, \\
a_{i i-1}=-\frac{c_{\varepsilon}}{\gamma_{i}^{2}}-\frac{p\left(x_{i}\right)}{h}, \quad i=2, \ldots, N-1,
\end{array}\right.
$$

and

$$
\left\{\begin{array}{l}
F_{1}(t)=f_{1}(t)-\left(\frac{c_{\varepsilon}}{\gamma_{1}^{2}}+\frac{p\left(x_{1}\right)}{h}\right) \phi(0, t) \\
F_{i}(t)=f_{i}(t), \quad i=2,3, \ldots, N-2 \\
F_{N-1}(t)=f_{N-1}(t)-\left(\frac{2 c_{\varepsilon}}{\gamma_{N-1}^{2}}\right) \psi(1, t)
\end{array}\right.
$$

Now we need to show the semi-discrete operator $L^{h}$ satisfies the maximum principle and the uniform stability estimate.

Lemma 3.1 (Semi-discrete maximum principle). Suppose $U_{0}(t) \geq 0, U_{N}(t) \geq 0$. Then $L^{h} U_{i}(t) \geq 0$ for all $i=1,2, \ldots, N-1$, implies that $U_{i}(t) \geq 0$ for all $i=0,1, \ldots, N$.

Proof. Suppose there exist $p \in\{0,1, \ldots, N\}$ such that $U_{p}(t)=\min _{0 \leq i \leq N} U_{i}(t)$. Suppose that $U_{p}(t)<0$, which implies $p \neq 0, N$. Also we have $U_{p+1}-U_{p}>0$ and $U_{p}-U_{p-1}<0$. Now, we have

$$
L^{h} U_{p}(t)=\frac{d U_{p}(t)}{d t}-c_{\varepsilon} \frac{U_{p+1}(t)-2 U_{p}(t)-U_{p-1}(t)}{\gamma_{p}^{2}}+p_{p} \frac{U_{p}(t)-U_{p-1}(t)}{h}+q_{p} U_{p}(t) .
$$

Using the assumption, we obtain that $L^{h} U_{i}(t)<0$ for $i=1,2, \ldots, N-1$. Thus, the supposition $U_{i}(t)<0, i=0,1, \ldots, N$ is wrong. Hence,

$$
U_{i}(t) \geq 0, \quad \text { for all } i=0,1, \ldots, N .
$$


Lemma 3.2. The solution $U_{i}(t)$ of the semi-discrete problem in (3.8) or (3.9) satisfy the following bound.

$$
\left|U_{i}(t)\right| \leq \theta^{-1} \max _{i}\left|L^{h} U_{i}(t)\right|+\max _{i}\left\{\left|u_{0}\left(x_{i}\right)\right|, \max _{i}\left\{\phi\left(x_{i}, t\right), \psi\left(x_{i}, t\right)\right\}\right\} .
$$

Proof. Let $p=\theta^{-1} \max _{i}\left|L^{h} U_{i}(t)\right|+\max _{i}\left\{\left|u_{0}\left(x_{i}\right)\right|, \max _{i}\left\{\phi\left(x_{i}, t\right), \psi\left(x_{i}, t\right)\right\}\right\}$ and we define the barrier function $\vartheta_{i}^{ \pm}(t)$ by

$$
\vartheta_{i}^{ \pm}(t)=p \pm U_{i}(t)
$$

At the boundary points

$$
\begin{aligned}
& \vartheta_{0}^{ \pm}(t)=p \pm u_{0}(t)=p \pm \phi(0, t) \geq 0, \\
& \vartheta_{N}^{ \pm}(t)=p \pm u_{N}(t)=p \pm \phi(1, t) \geq 0 .
\end{aligned}
$$

On the discretized domain $x_{i}, i=1,2, \ldots, N-1$, we have

$$
\begin{aligned}
L^{h} \vartheta_{i}^{ \pm}(t)= & \frac{d\left(p \pm U_{i}(t)\right)}{d t}-c_{\varepsilon}\left(\frac{p \pm U_{i+1}(t)-2\left(p \pm U_{i}(t)\right)+p \pm U_{i-1}(t)}{\gamma^{2}}\right) \\
& +p_{i}\left(\frac{p \pm U_{i}(t)-\left(p \pm U_{i-1}(t)\right)}{h}\right)+q_{i}\left(p \pm U_{i}(t)\right) \\
= & q_{i} p \pm L^{h} U_{i}(t) \\
= & q_{i}\left(\theta^{-1} \max _{i}\left|L^{h} U_{i}(t)\right|+\max _{i}\left\{\left|u_{0}\left(x_{i}\right)\right|, \max _{i}\left\{\phi\left(x_{i}, t\right), \psi\left(x_{i}, t\right)\right\}\right\}\right) \pm f_{i}(t) \\
\geq & 0, \quad \text { since } q_{i} \geq \theta .
\end{aligned}
$$

From Lemma 3.1, we obtain $\vartheta_{i}^{ \pm} \geq 0$, for all $\left(x_{i}, t\right) \in \bar{\Omega}_{x}^{N} \times \Omega_{t}$.

This complete the proof.

3.2. Convergence Analysis. Now, let us analyze the convergence of the spatial discretization. We proved above the semi-discrete operator $L^{h}$ satisfy the maximum principle and the uniform stability estimate. Note that $U_{i}(t)$ is denoted for the spatial semi-discretization approximate solution to the exact solution $u(x, t)$ at $x=x_{i}$, $i=0,1, \ldots, N$.

Let define the forward and backward finite differences in space as:

$$
D^{+} v\left(x_{i}, t\right)=\frac{v\left(x_{i+1}, t\right)-v\left(x_{i}, t\right)}{h}, \quad D^{-} v\left(x_{i}, t\right)=\frac{v\left(x_{i}, t\right)-v\left(x_{i-1}, t\right)}{h},
$$

respectively, and the second order finite difference operator as

$$
\delta^{2} v\left(x_{i}, t\right)=D^{+} D^{-} v\left(x_{i}, t\right)=\frac{D^{+} v\left(x_{i}, t\right)-D^{-} v\left(x_{i}, t\right)}{h} .
$$

Theorem 3.1. Let the coefficients functions $p(x), q(x)$ and the source function $f(x, t)$ in (2.3) be sufficiently smooth, so that $u(x, t) \in C^{4}[0,1] \times[0, T]$. Then the semi-discrete 
solution $U_{i}(t)$ of (2.3) satisfies

$$
\left|L^{h}\left(u\left(x_{i}, t\right)-U_{i}(t)\right)\right| \leq C h\left(1+\sup _{x \in(0,1)} \frac{\exp \left(-p^{*}(1-x) / c_{\varepsilon}\right)}{c_{\varepsilon}^{3}}\right) .
$$

Proof. We consider the truncation error in spatial discretization as:

$$
\begin{aligned}
& \left|L^{h}\left(u\left(x_{i}, t\right)-U_{i}(t)\right)\right| \\
= & \left|L^{h} u\left(x_{i}, t\right)-L^{h} U_{i}(t)\right| \\
\leq & C c_{\varepsilon}\left|\frac{\partial^{2} u}{\partial x^{2}}\left(x_{i}, t\right)-D_{x}^{+} D_{x}^{-} u\left(x_{i}, t\right)\right|+C c_{\varepsilon}\left|\left(\frac{h^{2}}{\gamma_{i}^{2}}-1\right) D_{x}^{+} D_{x}^{-} u\left(x_{i}, t\right)\right|+C h\left|\frac{\partial^{2} u}{\partial x^{2}}\left(x_{i}, t\right)\right| \\
\leq & C c_{\varepsilon} h^{2}\left|\frac{\partial^{4} u}{\partial x^{4}}\left(x_{i}, t\right)\right|+C h\left|\frac{\partial^{2} u}{\partial x^{2}}\left(x_{i}, t\right)\right|+C h\left|\frac{\partial^{2} u}{\partial x^{2}}\left(x_{i}, t\right)\right| \\
\leq & C c_{\varepsilon} h^{2}\left|\frac{\partial^{4} u}{\partial x^{4}}\left(x_{i}, t\right)\right|+C h\left|\frac{\partial^{2} u}{\partial x^{2}}\left(x_{i}, t\right)\right| .
\end{aligned}
$$

The bound $c_{\varepsilon}\left|\frac{h^{2}}{\gamma_{i}^{2}}-1\right| \leq C h$ used in above expression is based on the behavior of the denominator function $\left(\gamma_{i}^{2}\right)$ in non-standard finite difference. To illustrate the bound given there, let us define $\sigma=: p_{i} h / c_{\varepsilon}, \sigma \in(0, \infty)$. Then

$$
c_{\varepsilon}\left|\frac{h^{2}}{\gamma_{i}^{2}}-1\right|=p_{i} h\left|\frac{1}{\exp (\sigma)-1}-\frac{1}{\sigma}\right|=: p_{i} h R(\sigma) .
$$

By simplifying and writing explicitly we obtain

$$
R(\sigma)=\frac{\exp (\sigma)-\sigma-1}{\sigma(\exp (\sigma)-1)}
$$

and we obtain the limit is bounded as

$$
\lim _{\sigma \rightarrow 0} R(\sigma)=\frac{1}{2}, \quad \lim _{\sigma \rightarrow \infty} R(\sigma)=0 .
$$

Hence, for all $\sigma \in(0, \infty)$, we have $R(\sigma) \leq C$. So, the error estimate in the spatial discretization is bounded as

$$
\left|L^{h}\left(u\left(x_{i}, t\right)-U_{i}(t)\right)\right| \leq C c_{\varepsilon} h^{2}\left|\frac{\partial^{4} u}{\partial x^{4}}\left(x_{i}, t\right)\right|+C h\left|\frac{\partial^{2} u}{\partial x^{2}}\left(x_{i}, t\right)\right| .
$$

From (3.12) and boundedness of derivatives of solution in Lemma 2.4, we obtain

$$
\begin{aligned}
\left|L^{h}\left(u\left(x_{i}, t\right)-U_{i}(t)\right)\right| \leq & C c_{\varepsilon} h^{2}\left|1+c_{\varepsilon}^{-4} \exp \left(\frac{-p^{*}\left(1-x_{i}\right)}{c_{\varepsilon}}\right)\right| \\
& +C h\left|1+c_{\varepsilon}^{-2} \exp \left(\frac{-p^{*}\left(1-x_{i}\right)}{c_{\varepsilon}}\right)\right| \\
\leq & C h\left|1+\max _{i} c_{\varepsilon}^{-3} \exp \left(\frac{-p^{*}\left(1-x_{i}\right)}{c_{\varepsilon}}\right)\right| .
\end{aligned}
$$


Lemma 3.3. For a fixed mesh and for $\varepsilon \rightarrow 0$, it holds

$$
\lim _{c_{\varepsilon} \rightarrow 0} \max _{j} \frac{\exp \left(\frac{-p^{*}\left(1-x_{j}\right)}{c_{\varepsilon}}\right)}{c_{\varepsilon}^{m}}=0, \quad m=1,2,3, \ldots,
$$

where $x_{j}=j h, h=1 / N$, for all $j=1,2, \ldots, N-1$.

Proof. Let us consider the partition of the interval [0,1], with points $x_{j}=j h, h=$ $1 / N, j=0,1, \ldots, N$. By using $x_{1}=h, 1-x_{N-1}=h$, we have

$$
\begin{aligned}
\max _{1 \leq j \leq N-1} \frac{\exp \left(-p^{*} x_{j} / c_{\varepsilon}\right)}{c_{\varepsilon}^{m}} & \leq \frac{\exp \left(-p^{*} x_{1} / c_{\varepsilon}\right)}{c_{\varepsilon}^{m}}=\frac{\exp \left(-p^{*} h / c_{\varepsilon}\right)}{c_{\varepsilon}^{m}} \text { and } \\
\max _{1 \leq j \leq N-1} \frac{\exp \left(-p^{*}\left(1-x_{j}\right) / c_{\varepsilon}\right)}{c_{\varepsilon}^{m}} & \leq \frac{\exp \left(-p^{*}\left(1-x_{N-1}\right) / c_{\varepsilon}\right)}{c_{\varepsilon}^{m}}=\frac{\exp \left(-p^{*} h / c_{\varepsilon}\right)}{c_{\varepsilon}^{m}}
\end{aligned}
$$

Then, by the repeated application of L'Hospital's rule, we have

$$
\lim _{c_{\varepsilon} \rightarrow 0} \frac{\exp \left(-p^{*} h / c_{\varepsilon}\right)}{c_{\varepsilon}^{m}}=\lim _{r=1 / c_{\varepsilon} \rightarrow \infty} \frac{r^{m}}{\exp \left(p^{*} h r\right)}=\lim _{r=1 / c_{\varepsilon} \rightarrow \infty} \frac{m !}{\left(p^{*} h\right)^{m} \exp \left(p^{*} h r\right)}=0 .
$$

Hence, the proof is completed.

Theorem 3.2. Under the hypothesis of boundedness of semi-discrete solution (i.e., it satisfies the semi-discrete maximum principle), Lemma 3.3 and Theorem 3.1, the semi-discrete solution satisfy the following bound.

$$
\sup _{0<c_{\varepsilon} \ll 1} \max _{i}\left|u\left(x_{i}, t\right)-U_{i}(t)\right|_{\Omega_{x}^{N} \times \Omega_{t}} \leq C N^{-1}
$$

Proof. Results from boundedness of solution, Lemma 3.1 and Theorem 3.1 gives the required estimates.

3.3. Discretization in temporal direction. On the time domain $\Omega_{t}=[0, T]$, we construct a mesh with mesh length $\Delta t_{j}=t_{j+1}-t_{j}, j=0,1,2, \ldots, M-1$, where $M$ denotes the number of mesh points in time direction. First rewrite (3.9) in the form

$$
\frac{d U_{i}(t)}{d t}=f\left(t, U_{i}(t)\right)
$$

with the initial condition $U\left(x_{i}, 0\right)=u_{0}\left(x_{i}\right), i=0,1, \ldots, N$, where $f\left(t, U_{i}(t)\right)=$ $-A U_{i}(t)+F_{i}(t)$. Now, we solve the system of IVPs in (3.15) using implicit RungeKutta method of order 2 and 3 given in [7]. 
We construct the scheme for each $j=0,1,2, \ldots, M-1$, as (3.16)

$$
\left\{\begin{array}{l}
k_{1}=f\left(t_{j}, U_{i, j}\right) \\
k_{2}=f\left(t_{j}+\frac{1}{2} \Delta t_{j}, U_{i, j}+\frac{1}{2} \Delta t_{j} k_{1}\right) \\
k_{3}=f\left(t_{j}+\frac{3}{4} \Delta t_{j}, U_{i, j}+\frac{3}{4} \Delta t_{j} k_{2}\right) \\
U_{i, j+1}^{*}=U_{i, j}+\frac{2}{9} \Delta t_{j} k_{1}+\frac{1}{3} \Delta t_{j} k_{2}+\frac{4}{9} \Delta t_{j} k_{3}, \\
k_{4}=f\left(t_{j}+\Delta t_{j}, U_{i, j+1}^{*}\right), \\
U_{i, j+1}=U_{i, j}+\frac{7}{24} \Delta t_{j} k_{1}+\frac{1}{4} \Delta t_{j} k_{2}+\frac{1}{3} \Delta t_{j} k_{3}+\frac{1}{8} \Delta t_{j} k_{4}, \quad i=1,2, \ldots, N-1
\end{array}\right.
$$

For each $j$ the local approximation $U_{i, j+1}$ to $U_{i}\left(t_{j+1}\right)$ has third order (i.e., $O\left(\Delta t_{j}\right)^{3}$ ) accuracy [7]. Let denote $\Delta t=\max _{j} \Delta t_{j}$.

Lemma 3.4. From the approximation method in temporal direction, the global error estimates in this direction is given by

$$
\left\|E_{j+1}\right\|=\max _{j}\left|U_{i}\left(t_{j+1}\right)-U_{i, j+1}\right|_{\Omega_{x}^{N} \times \Omega_{t}^{M}} \leq C(\Delta t)^{2},
$$

where $\left\|E_{j+1}\right\|$ is the global error in the temporal direction at $(j+1)^{\text {th }}$ time level.

Proof. Using the local error estimate upto $j$ th time step, we obtain the global error estimate at $(j+1)$ th time step.

$$
\begin{aligned}
\left\|E_{j+1}\right\| & =\left\|\sum_{l=1}^{j} e_{l}\right\| \quad(j \leq M) \\
& \leq\left\|e_{1}\right\|+\left\|e_{2}\right\|+\cdots+\left\|e_{j}\right\| \quad\left(\left\|e_{l}\right\|=C_{l}\left(\Delta t_{j}\right)^{3}\right) \\
& \leq C_{1}(j \Delta t)(\Delta t)^{2} \\
& \leq C_{1} T(\Delta t)^{2} \quad(\text { since } j \Delta t \leq T) \\
& \leq C(\Delta t)^{2} .
\end{aligned}
$$

Hence,

$$
\left\|E_{j+1}\right\|=\max _{j}\left|U_{i}\left(t_{j+1}\right)-U_{i, j+1}\right| \leq C(\Delta t)^{2} .
$$

Here, since $C$ and $\Delta t$ are independent of the perturbation parameter $\varepsilon$. By taking the suprimum for all $\varepsilon \in(0,1]$ we obtain

$$
\sup _{0<c_{\varepsilon} \ll 1} \max _{j}\left|U_{i}\left(t_{j+1}\right)-U_{i, j+1}\right|_{\Omega_{x}^{N} \times \Omega_{t}^{M}} \leq C(\Delta t)^{2} .
$$

This shows that the discretization in time direction is consistent and global error is bounded, with the error bound $O(\Delta t)^{2}$. 
Now, we use (3.14) and (3.18) to prove the $\varepsilon$-uniform convergence of the fully discrete scheme as

$$
\begin{aligned}
\sup _{0<c_{\varepsilon} \ll 1} \max _{i, j}\left|u\left(x_{i}, t_{j}\right)-U_{i, j}\right|= & \sup _{0<c_{\varepsilon} \ll 1} \max _{i, j}\left|u\left(x_{i}, t_{j}\right)-U_{i}\left(t_{j}\right)+U_{i}\left(t_{j}\right)-U_{i, j}\right| \\
\leq & \sup _{0<c_{\varepsilon} \ll 1} \max _{i, j}\left|u\left(x_{i}, t_{j}\right)-U_{i}\left(t_{j}\right)\right| \\
& +\sup _{0<c_{\varepsilon} \ll 1} \max _{i, j}\left|U_{i}\left(t_{j}\right)-U_{i, j}\right| .
\end{aligned}
$$

Hence, we obtain the required bound as

$$
\sup _{0<c_{\varepsilon} \ll 1}\left\|u\left(x_{i}, t_{j}\right)-U_{i, j}\right\|_{\Omega_{x}^{N} \times \Omega_{t}^{M}} \leq C\left(N^{-1}+(\Delta t)^{2}\right) .
$$

Remark 3.1. The inequality in (3.19) shows the $\varepsilon$-uniform convergence of the scheme with order of convergence: first order in spatial direction and second order in temporal direction.

\section{Numerical Results and Discussion}

To validate the established theoretical results, we perform some experiments using the proposed numerical scheme on the problem given in (2.1). We consider two numerical examples to verify the $\varepsilon$-convergence of the proposed scheme.

Example 4.1. From [20]

$$
\frac{\partial u}{\partial t}-\varepsilon^{2} \frac{\partial^{2} u}{\partial x^{2}}+\left(2+x+x^{2}\right) \frac{\partial u}{\partial x}+\left(\frac{1+x^{2}}{2}\right) u(x-\delta, t)=\sin (\pi x(1-x)),
$$

with $T=1$ subject to the initial condition $u(x, 0)=0, x \in[0,1]$, and intervalboundary conditions $\phi(x, t)=0,-\delta \leq x \leq 0, \psi(1, t)=0$ on $t \in[0,1]$.

Example 4.2. From [9] by setting $\eta=0$

$$
\frac{\partial u}{\partial t}-\varepsilon^{2} \frac{\partial^{2} u}{\partial x^{2}}+\frac{\partial u}{\partial x}+\left(2-x^{2}\right) u(x-\delta, t)+\left(1+x^{2}+\exp (x)\right) u(x, t)=50(x(1-x))^{3},
$$

with $T=2$ subject to the initial condition $u(x, 0)=0, x \in[0,1]$, and interval-boundary conditions $\phi(x, t)=0,-\delta \leq x \leq 0, \psi(1, t)=0$ on $t \in[0,2]$.

Exact solution is not available for these two problems, therefore maximum nodal errors are calculated by using the double mesh technique given in [9] as

$$
E_{\varepsilon, \delta}^{N, M}=\max _{i, j}\left|U_{i, j}^{N, M}-U_{i, j}^{2 N, 2 M}\right|,
$$

where $N$ and $M$ are the number of mesh points in $x$ and $t$, respectively. $U_{i, j}^{N, M}$ are the computed solutions of the problem using $N, M$ mesh numbers and $U_{i, j}^{2 N, 2 M}$ are computed solutions on double number of mesh points $2 N, 2 M$ by including the mid points $x_{i+1 / 2}$ and $t_{j+1 / 2}$ into the mesh points. For any value of the mesh points $N, M$ the $\varepsilon$-uniform error estimate are calculated by

$$
E^{N, M}=\max _{\varepsilon, \delta}\left|E_{\varepsilon, \delta}^{N, M}\right| \text {. }
$$


The rate of convergence of the method is given by

$$
r_{\varepsilon, \delta}^{N, M}=\log _{2}\left(E_{\varepsilon, \delta}^{N, M} / E_{\varepsilon, \delta}^{2 N, 2 M}\right)=\frac{\log \left(E_{\varepsilon, \delta}^{N, M}\right)-\log \left(E_{\varepsilon, \delta}^{2 N, 2 M}\right)}{\log 2}
$$

and the $\varepsilon$-uniform convergence is calculated by

$$
r^{N, M}=\log _{2}\left(E^{N, M} / E^{2 N, 2 M}\right)=\frac{\log \left(E^{N, M}\right)-\log \left(E^{2 N, 2 M}\right)}{\log 2} .
$$

The solutions of Examples 4.1 and 4.2 have a boundary layer on the right side of the $x$-domain as shown in Figures 3 and 5 . The effect of perturbation parameter $\varepsilon$ and delay parameter $\delta$ is shown in Figures 1 and 2, by using different values for $\varepsilon$ and $\delta$ for the test problems, i.e., one can observe from Figure 1 (a) and 2 (a), when the perturbation parameter tends to zero strong boundary layer is formed on the right side of the $x$-domain, in Figures 1 (b) and 2 (b) we observe that as the value of the delay parameter increases the thickness of the boundary layer decreases. The numerical results displayed in Tables 1 and 3 indicate the proposed method is convergent independent of the perturbation parameter. From the results in tables $1-3$ and Figure 4, one can observe that the maximum point-wise error $E_{\varepsilon, \delta}^{N, M}$ decreases as $\mathrm{N}$ increases for each value of $\varepsilon$. in addition to that, the maximum point-wise error is stable as $\varepsilon \rightarrow 0$ for each $N, M$. Using the results in these two examples we confirm the proposed numerical method is more accurate, stable and $\varepsilon$-uniform convergent with rate of convergence one. Numerical results in tables and figure confirm the parameter-uniformness of the proposed scheme. The results in this method are better than that obtained in [20] and [19].

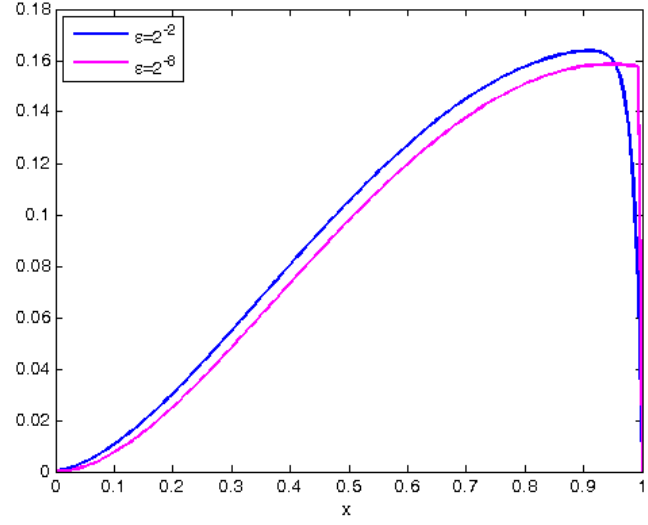

(a)

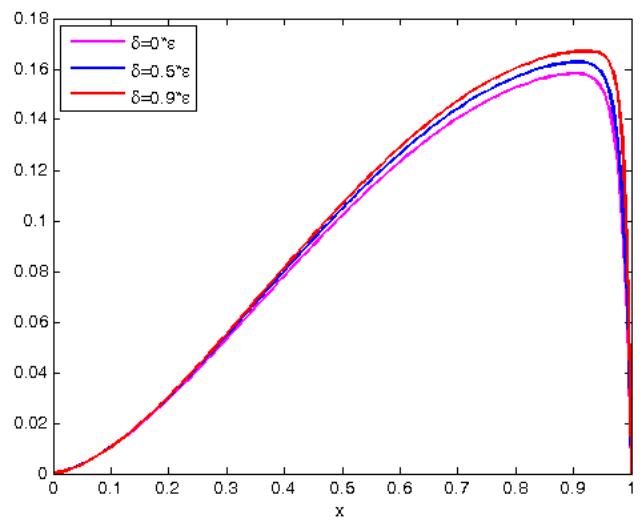

(b)

FiguRE 1. Example 4.1, in (a) the solution behavior for different values of $\varepsilon$ at $T=1$, in (b) the behavior of solution for different values of delay at $\varepsilon=2^{-2}$ and $T=1$. 
TABLE 1. Example 4.1 maximum absolute error and rate of convergence for the proposed method and results in [20] with $\delta=0.6 \varepsilon$

\begin{tabular}{|c|c|c|c|c|c|c|}
\hline$\varepsilon$ & $\mathrm{N}=32$ & 64 & 128 & 256 & 512 & 1024 \\
\hline$\downarrow$ & $\mathrm{M}=60$ & 120 & 240 & 480 & 960 & 1920 \\
\hline \multicolumn{7}{|l|}{ Our } \\
\hline \multicolumn{7}{|l|}{ Scheme } \\
\hline \multirow{2}{*}{$2^{-6}$} & $3.9367 \mathrm{e}-03$ & $1.9697 \mathrm{e}-03$ & $9.8547 \mathrm{e}-04$ & $4.9293 \mathrm{e}-04$ & $2.4651 \mathrm{e}-04$ & $1.2327 \mathrm{e}-04$ \\
\hline & 0.9990 & 0.9991 & 0.9994 & 0.9997 & 0.9998 & - \\
\hline \multirow[t]{2}{*}{$2^{-8}$} & $3.9308 \mathrm{e}-03$ & $1.9667 \mathrm{e}-03$ & $9.8399 \mathrm{e}-04$ & $4.9219 \mathrm{e}-04$ & $2.4614 \mathrm{e}-04$ & $1.2308 \mathrm{e}-04$ \\
\hline & 0.9990 & 0.9991 & 0.9994 & 0.9997 & 0.9999 & - \\
\hline \multirow[t]{2}{*}{$2^{-10}$} & $3.9293 \mathrm{e}-03$ & $1.9660 \mathrm{e}-03$ & $9.8362 \mathrm{e}-04$ & $4.9201 \mathrm{e}-04$ & $2.4605 \mathrm{e}-04$ & $1.2304 \mathrm{e}-04$ \\
\hline & 0.9990 & 0.9991 & 0.9994 & 0.9997 & 0.9998 & - \\
\hline \multirow[t]{2}{*}{$2^{-12}$} & $3.9289 \mathrm{e}-03$ & $1.9658 \mathrm{e}-03$ & $9.8353 \mathrm{e}-04$ & $4.9196 \mathrm{e}-04$ & 2.4603e-04 & $1.2303 \mathrm{e}-04$ \\
\hline & 0.9990 & & & 0.9997 & 0.9998 & - \\
\hline \multirow[t]{2}{*}{$2^{-14}$} & $3.9288 \mathrm{e}-03$ & $1.9658 \mathrm{e}-03$ & $9.8351 \mathrm{e}-04$ & $4.9195 \mathrm{e}-04$ & 2.4602e-04 & $1.4180 \mathrm{e}-04$ \\
\hline & 0.9990 & 0.9991 & 0.9994 & 0.9997 & 0.7949 & - \\
\hline \multirow[t]{2}{*}{$2^{-16}$} & $3.9288 \mathrm{e}-03$ & $1.9658 \mathrm{e}-03$ & $9.8350 \mathrm{e}-04$ & $4.9195 \mathrm{e}-04$ & $2.4602 \mathrm{e}-04$ & $1.2302 \mathrm{e}-04$ \\
\hline & 0.9990 & 0.99 & 0.9994 & 0.9997 & 0.9999 & - \\
\hline \multirow[t]{2}{*}{$2^{-18}$} & $3.9288 \mathrm{e}-03$ & $1.9657 \mathrm{e}-03$ & $9.8350 \mathrm{e}-04$ & $4.9194 \mathrm{e}-04$ & $2.4602 \mathrm{e}-04$ & $1.2302 \mathrm{e}-04$ \\
\hline & 0.9990 & & & & & 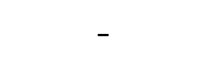 \\
\hline \multirow[t]{2}{*}{$2^{-20}$} & $3.9288 \mathrm{e}-03$ & $1.9657 \mathrm{e}-03$ & $9.8350 \mathrm{e}-04$ & $4.9194 \mathrm{e}-04$ & $2.4602 \mathrm{e}-04$ & $1.2302 \mathrm{e}-04$ \\
\hline & 0.9990 & 0.9991 & 0.9994 & 0.9997 & 0.9999 & - \\
\hline \multicolumn{7}{|l|}{$\begin{array}{l}\text { Result } \\
\text { in }[20]\end{array}$} \\
\hline \multirow[t]{2}{*}{$2^{-6}$} & $5.9208 \mathrm{e}-03$ & $3.6201 \mathrm{e}-03$ & $2.1189 \mathrm{e}-03$ & $1.1966 \mathrm{e}-03$ & $6.5640 \mathrm{e}-04$ & $3.5122 \mathrm{e}-04$ \\
\hline & 0.70 & & & & 0.9022 & 0.9703 \\
\hline \multirow[t]{2}{*}{$2^{-8}$} & $5.9146 \mathrm{e}-03$ & $3.6167 \mathrm{e}-03$ & $2.1172 \mathrm{e}-03$ & $1.1959 \mathrm{e}-03$ & $6.5607 \mathrm{e}-04$ & $3.5165 \mathrm{e}-04$ \\
\hline & 0.7096 & 0.7725 & 0.8241 & 0.8662 & 0.8997 & 0.9256 \\
\hline \multirow[t]{2}{*}{$2^{-10}$} & $5.9131 \mathrm{e}-03$ & $3.6158 \mathrm{e}-03$ & $2.1168 \mathrm{e}-03$ & $1.1957 \mathrm{e}-03$ & $6.5597 \mathrm{e}-04$ & $3.5161 \mathrm{e}-04$ \\
\hline & 0.7096 & 0.7724 & & & 0.8997 & 0.9256 \\
\hline \multirow[t]{2}{*}{$2^{-12}$} & $5.9127 \mathrm{e}-03$ & $3.6156 \mathrm{e}-03$ & $2.1167 \mathrm{e}-03$ & $1.1957 \mathrm{e}-03$ & $6.5594 \mathrm{e}-04$ & $3.5160 \mathrm{e}-04$ \\
\hline & 0.7096 & 0.7724 & 0.8240 & & 0.8996 & 0.9256 \\
\hline \multirow[t]{2}{*}{$2^{-14}$} & $5.9126 \mathrm{e}-03$ & $3.6155 \mathrm{e}-03$ & $2.1167 \mathrm{e}-03$ & $1.1957 \mathrm{e}-03$ & $6.5594 \mathrm{e}-04$ & $3.5160 \mathrm{e}-04$ \\
\hline & 0.7096 & 0.7724 & 0.8240 & 0.8662 & 0.8996 & 0.9256 \\
\hline \multirow[t]{2}{*}{$2^{-16}$} & $5.9126 \mathrm{e}-03$ & $3.6155 \mathrm{e}-03$ & $2.1167 \mathrm{e}-03$ & $1.1957 \mathrm{e}-03$ & $6.5594 \mathrm{e}-04$ & $3.5160 \mathrm{e}-04$ \\
\hline & 0.7096 & & & & & 0.9256 \\
\hline \multirow[t]{2}{*}{$2^{-18}$} & $5.9126 \mathrm{e}-03$ & $3.6155 \mathrm{e}-03$ & $2.1167 \mathrm{e}-03$ & $1.1957 \mathrm{e}-03$ & $6.5593 \mathrm{e}-04$ & $3.5160 \mathrm{e}-04$ \\
\hline & 0.7096 & 0.7724 & 0.8240 & 0.8662 & 0.8996 & 0.9256 \\
\hline \multirow[t]{2}{*}{$2^{-20}$} & $5.9125 \mathrm{e}-03$ & $3.6155 \mathrm{e}-03$ & $2.1167 \mathrm{e}-03$ & $1.1957 \mathrm{e}-03$ & 6.5593e-04 & $3.5160 \mathrm{e}-04$ \\
\hline & 0.7096 & 0.7724 & 0.8240 & 0.8662 & 0.8996 & 0.9256 \\
\hline
\end{tabular}


TABLE 2. Example 4.1's $\varepsilon$-uniform absolute error and rate of convergence for the proposed method and results in [20] and (in [19] for the case $\delta=0.9 \varepsilon)$.

\begin{tabular}{|c|c|c|c|c|c|c|}
\hline $\mathrm{N}$ : & 32 & 64 & 128 & 256 & 512 & 1024 \\
\hline M: & 60 & 120 & 240 & 480 & 960 & 1920 \\
\hline \multicolumn{7}{|l|}{ Our } \\
\hline \multicolumn{7}{|l|}{ Scheme. } \\
\hline$E^{N, M}$ & $3.9288 \mathrm{e}-03$ & $1.9657 \mathrm{e}-03$ & $9.8350 \mathrm{e}-04$ & $4.9194 \mathrm{e}-04$ & $2.4602 \mathrm{e}-04$ & $1.2302 \mathrm{e}-04$ \\
\hline$r^{N, M}$ & 0.9990 & 0.9991 & 0.9994 & 0.9997 & 0.9999 & - \\
\hline \multicolumn{7}{|l|}{$\begin{array}{l}\text { Result } \\
\text { in }[20]\end{array}$} \\
\hline$E^{N, M}$ & $5.9125 \mathrm{e}-03$ & $3.6155 \mathrm{e}-03$ & $2.1167 \mathrm{e}-03$ & $1.1957 \mathrm{e}-03$ & $6.5593 \mathrm{e}-04$ & $3.5160 \mathrm{e}-04$ \\
\hline$r^{N, M}$ & 0.7096 & 0.7724 & 0.8240 & 0.8662 & 0.8996 & 0.9256 \\
\hline \multicolumn{7}{|l|}{$\begin{array}{l}\text { Result } \\
\text { in [19] }\end{array}$} \\
\hline$E^{M, N}$ & $8.3467 \mathrm{e}-03$ & $5.3894 \mathrm{e}-03$ & $3.3649 \mathrm{e}-03$ & $2.0082 \mathrm{e}-03$ & $1.1571 \mathrm{e}-03$ & $6.4904 \mathrm{e}-04$ \\
\hline$r^{M, N}$ & 0.6311 & 0.6796 & 0.7447 & 0.7954 & 0.8341 & - \\
\hline
\end{tabular}

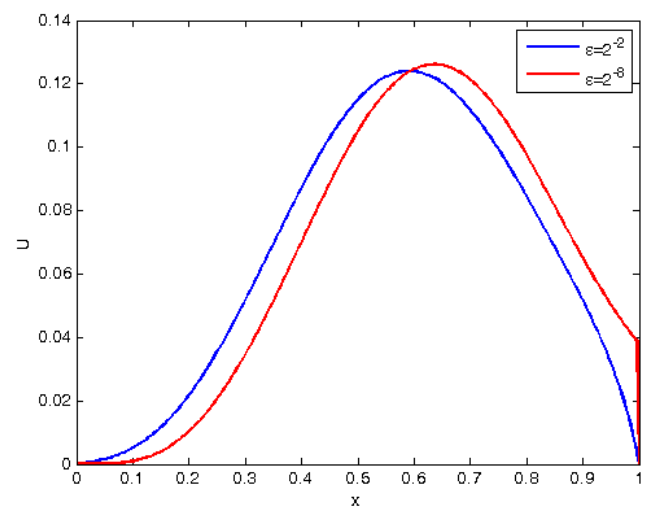

(a)

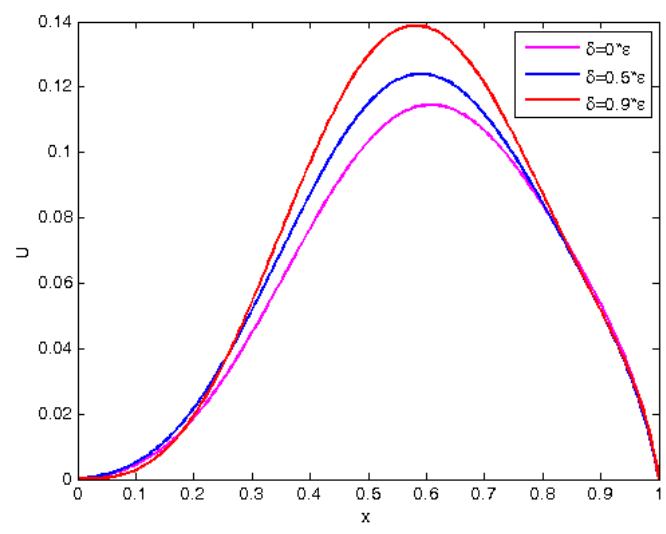

FiguRE 2. Example 4.2, in (a) the solution behavior for different values of $\varepsilon$ at $T=2$, in (b) the behavior of solution for different values of delay at $\varepsilon=2^{-2}$ and $T=2$.

\section{Conclusions}

A numerical method is developed for solving a singularly perturbed parabolic delay differential equation whose solution exhibit a boundary layer behavior. The developed method is based on method of line that constitute the non-standard finite difference for the spatial discretization and the Runge-Kutta order 2 and 3 implicit method in the temporal direction for the system of IVP resulting from the spatial discretization. Stability and convergence analysis of the proposed scheme is shown. The applicability 
TABLE 3. Example 4.2's maximum absolute error and rate of convergence for the proposed method with $\delta=0.5 \varepsilon$

\begin{tabular}{|c|c|c|c|c|c|c|}
\hline $\begin{array}{l}\varepsilon \\
\downarrow\end{array}$ & $\mathrm{N}=\mathrm{M}=32$ & 64 & 128 & 256 & 512 & 1024 \\
\hline \multicolumn{7}{|l|}{ Our } \\
\hline \multicolumn{7}{|l|}{ Scheme } \\
\hline \multirow[t]{2}{*}{$2^{-6}$} & $5.7389 \mathrm{e}-03$ & $2.9180 \mathrm{e}-03$ & $1.4718 \mathrm{e}-03$ & 7.3915e-04 & $3.7036 \mathrm{e}-04$ & $1.8537 \mathrm{e}-04$ \\
\hline & 0.9758 & 0.9874 & 0.9936 & 0.9969 & 0.9985 & - \\
\hline \multirow[t]{2}{*}{$2^{-8}$} & $5.7126 \mathrm{e}-03$ & $2.9034 \mathrm{e}-03$ & $1.4647 \mathrm{e}-03$ & $7.3552 \mathrm{e}-04$ & $3.6854 \mathrm{e}-04$ & $1.8447 \mathrm{e}-04$ \\
\hline & 0.9764 & 0.9871 & 0.9938 & 0.9969 & 0.9984 & - \\
\hline \multirow[t]{2}{*}{$2^{-10}$} & $5.7061 \mathrm{e}-03$ & $2.8998 \mathrm{e}-03$ & $1.4630 \mathrm{e}-03$ & $7.3461 \mathrm{e}-04$ & $3.6808 \mathrm{e}-04$ & $1.8424 \mathrm{e}-04$ \\
\hline & 0.9766 & 0.9870 & 0.9939 & 0.9970 & 0.9984 & - \\
\hline \multirow[t]{2}{*}{$2^{-12}$} & $5.7044 \mathrm{e}-03$ & $2.8989 \mathrm{e}-03$ & $1.4625 \mathrm{e}-03$ & $7.3438 \mathrm{e}-04$ & $3.6797 \mathrm{e}-04$ & $1.8418 \mathrm{e}-04$ \\
\hline & 0.9766 & 0.9871 & 0.9938 & 0.9969 & 0.9985 & - \\
\hline \multirow[t]{2}{*}{$2^{-14}$} & $5.7040 \mathrm{e}-03$ & $2.8987 \mathrm{e}-03$ & $1.4624 \mathrm{e}-03$ & 7.3433e-04 & $3.6794 \mathrm{e}-04$ & $1.8417 \mathrm{e}-04$ \\
\hline & 0.9766 & 0.9871 & 0.9938 & 0.9970 & 0.9984 & - \\
\hline \multirow[t]{2}{*}{$2^{-16}$} & $5.7039 \mathrm{e}-03$ & $2.8986 \mathrm{e}-03$ & $1.4624 \mathrm{e}-03$ & $7.3431 \mathrm{e}-04$ & $3.6794 \mathrm{e}-04$ & $1.8417 \mathrm{e}-04$ \\
\hline & 0.9766 & 0.9870 & 0.9939 & 0.9969 & 0.9984 & - \\
\hline \multirow[t]{2}{*}{$2^{-18}$} & $5.7039 \mathrm{e}-03$ & $2.8986 \mathrm{e}-03$ & $1.4624 \mathrm{e}-03$ & $7.3431 \mathrm{e}-04$ & $3.6793 \mathrm{e}-04$ & $1.8416 \mathrm{e}-04$ \\
\hline & 0.9766 & 0.9870 & 0.9939 & 0.9970 & 0.9985 & - \\
\hline \multirow[t]{2}{*}{$2^{-20}$} & $5.7039 \mathrm{e}-03$ & $2.8986 \mathrm{e}-03$ & $1.4624 \mathrm{e}-03$ & $7.3431 \mathrm{e}-04$ & $3.6793 \mathrm{e}-04$ & $1.8416 \mathrm{e}-04$ \\
\hline & 0.9766 & 0.9870 & 0.9939 & 0.9970 & 0.9985 & - \\
\hline$E^{N, M}$ & $5.7389 \mathrm{e}-03$ & $2.9180 \mathrm{e}-03$ & $1.4718 \mathrm{e}-03$ & 7.3915e-04 & $3.7036 \mathrm{e}-04$ & $1.8537 \mathrm{e}-04$ \\
\hline$r^{N, M}$ & 0.9758 & 0.9874 & 0.9936 & 0.9969 & 0.9985 & - \\
\hline
\end{tabular}
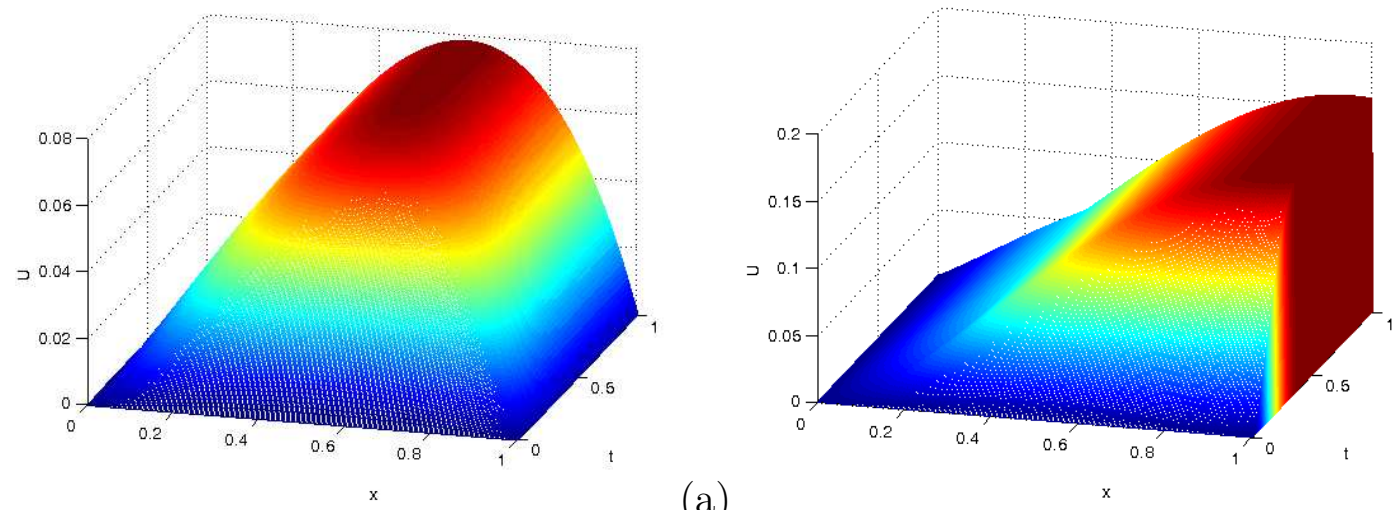

(a)

FiguRE 3. Example 4.1, (a) numerical solution for $\varepsilon=1, \delta=0.6 \varepsilon$ and (b) numerical solution for $\varepsilon=2^{-10}, \delta=0.6 \varepsilon$. 


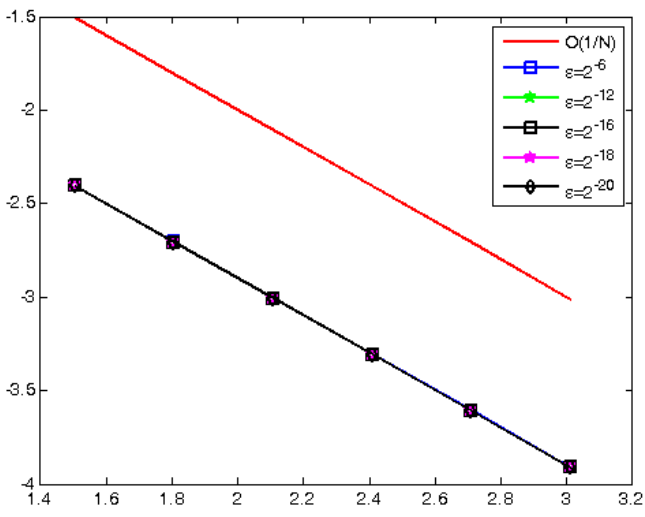

(a)

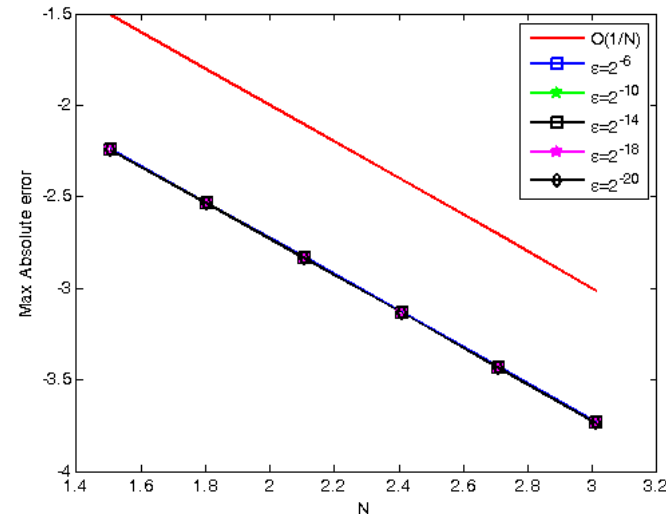

(b)

Figure 4. Example 4.1 on left and Example 4.2 on right, Log-Log scale plot of the maximum absolute error for different values of $\varepsilon$.

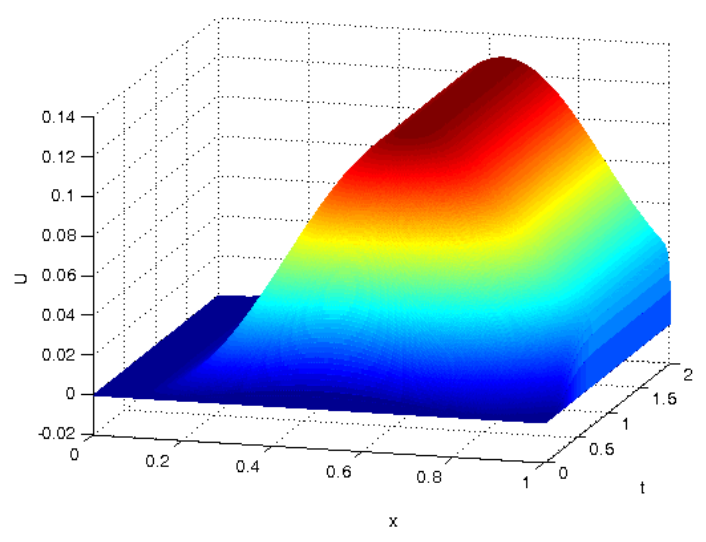

(a)

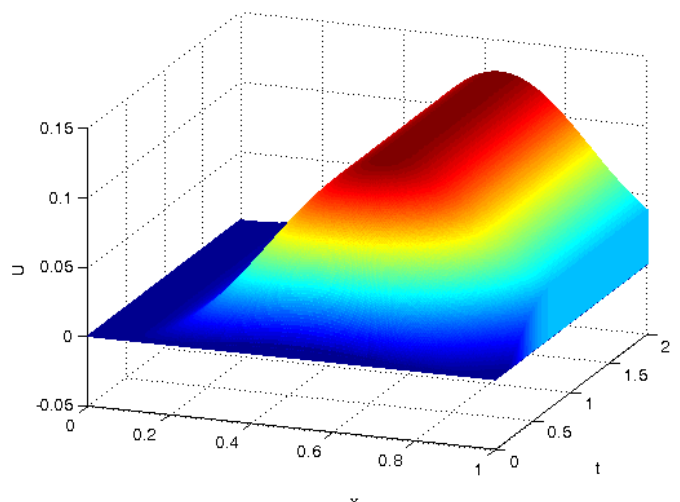

(b)

FiguRE 5. Example 4.2, in (a) numerical solution for $\varepsilon=2^{-4}, \delta=0.5 \varepsilon$ and in (b) numerical solution for $\varepsilon=2^{-20}, \delta=0.5 \varepsilon$.

of the proposed scheme is investigated by taking two examples. The effect of the perturbation parameter and the delay on the solution of the problem are shown by using figures. The method is shown to be $\varepsilon$-uniformly convergent with order of convergence $O\left(N^{-1}+(\Delta t)^{2}\right)$. The performance of the proposed scheme is investigated by comparing with prior study. The proposed method gives more accurate, stable and $\varepsilon$-uniform numerical result.

Acknowledgements. The authors would like to acknowledge the reviewers in advance for their constructive comments. 


\section{REFERENCES}

[1] C. T. H. Baker, G. A. Bocharov and F. A Rihan, A report on the use of delay differential equations in numerical modelling in the biosciences, Department of Mathematics, University of Manchester, Manchester, England, 1999.

[2] K. Bansal and K. K. Sharma, $\varepsilon$-Uniform numerical technique for the class of time dependent singularly perturbed parabolic problems with state dependent retarded argument arising from generalised Stein's model of neuronal variability, Differ. Equ. Dyn. Syst. (2016), 1-28.

[3] K. Bansal and K. K. Sharma, Parameter uniform numerical scheme for time dependent singularly perturbed convection-diffusion-reaction problems with general shift arguments, Numer. Algorithms 75(1) (2017), 113-145.

[4] K. Bansal, P. Rai and K. K. Sharma, Numerical treatment for the class of time dependent singularly perturbed parabolic problems with general shift arguments, Differ. Equ. Dyn. Syst. 25(2) (2017), 327-346.

[5] K. Bansal and K. K. Sharma, Parameter-robust numerical scheme for time-dependent singularly perturbed reaction-diffusion problem with large delay, Numer. Funct. Anal. Optim. 39(2) (2018), $127-154$.

[6] A. Bellen and M. Zennaro, Numerical Methods for Delay Differential Equations, Clarendon Press, Oxford, 2003.

[7] P. Bogacki and L. F. Shampine, A 3(2) pair of Runge-Kutta formulas, Appl. Math. Lett. 2(4) (1989), 321-325.

[8] V. Y. Glizer, Asymptotic analysis and solution of a finite-horizon $H^{\infty}$ control problem for singularly-perturbed linear systems with small state delay, J. Optim. Theory Appl. 117(2) (2003), $295-325$.

[9] V. Gupta, M. Kumar and S. Kumar, Higher order numerical approximation for time dependent singularly perturbed differential-difference convection-diffusion equations, Numer. Methods Partial Differential Equations 34 (2018), 357-380.

[10] T. Kellogg, Analysis of some difference approximations for a singular perturbation problem without turning point, Math. Comput. 32 (1978), 1025-1039.

[11] Y. Kuang, Delay Differential Equations: With Applications in Population Dynamics, Academic Press, Boston, San Diego, New York, 1993.

[12] D. Kumar and M. K. Kadalbajoo, A parameter-uniform numerical method for time-dependent singularly perturbed differential-difference equations, Appl. Math. Model. 35(6) (2011), 28052819.

[13] R. Kumar and S. Kumar, Convergence of three-step Taylor galerkin finite element scheme based monotone schwarz iterative method for singularly perturbed differential-difference equation, Numer. Funct. Anal. Optim. 36(8) (2015), 1029-1045.

[14] C. G. Lange and R. M. Miura, Singular perturbation analysis of boundary value problems for differential-difference equations III. Turning point problems, SIAM J. Appl. Math. 45(5) (1985), 708-734.

[15] C. G. Lange and R. M. Miura, Singular perturbation analysis of boundary value problems for differential-difference equations. V. Small shifts with layer behavior, SIAM J. Appl. Math. 54(1) (1994), 249-272.

[16] P. R. Mahaffy, M. F. A'Hearn, S. K. Atreya, et.al., The Champollion cometary molecular analysis experiment, Advances in Space Research 23(2) (1999), 349-359.

[17] R. E. Mickens, Advances in the Applications of Nonstandard Finite Diffference Schemes, World Scientific, Singapure, New Jersey, London, Hong Kong, 2005.

[18] M. Musila and P. Lánskỳ, Generalized Stein's model for anatomically complex neurons, Biosystems 25(3) (1991), 179-191. 
[19] V. P. Ramesh and M. K. Kadalbajoo, Upwind and midpoint upwind difference methods for timedependent differential difference equations with layer behavior, Appl. Math. Comput. 202(2) (2008), 453-471.

[20] R. N. Rao and P. P. Chakravarthy, Fitted numerical methods for singularly perturbed $1 D$ parabolic partial differential equations with small shifts arising in the modelling of neuronal variability, Differ. Equ. Dyn. Syst. (2017), 1-18.

[21] H. G. Roos, M. Stynes and L. R. Tobiska, Numerical Methods for Singularly Perturbed Differential Equations, Springer-Verlag, Berlin, 2008.

[22] R. B. Stein, A theoretical analysis of neuronal variability, Biophysical Journal 5(2) (1965), 173-194.

[23] R. B. Stein, Some models of neuronal variability, Biophysical journal 7(1) (1967), 37-68.

[24] H. Tian, The exponential asymptotic stability of singularly perturbed delay differential equations with a bounded lag, J. Math. Anal. Appl. 270(1) (2002), 143-149.

[25] H. C. Tuckwell, Stochastic Processes in the Neurosciences, SIAM, Philadelphia, 1989.

[26] H. C. Tuckwell, Introduction to Theoretical Neurobiology: Linear cable theory and dendritic structure, Cambridge University Press, Cambridge, 2005.

[27] J. Wu, Theory and Applications of Partial Functional Differential Equations, Springer-Verlag, New York, 2012.

${ }^{1}$ Department of Mathematics, College of Natural Sciences, JIMMA UNIVERSITY, JimMA, ETHIOPIA

Email address: msfnmkr02@gmail.com

Email address: gammeef@gmail.com 
Kragujevac Journal of Mathematics

Volume 46(1) (2022), Pages 85-104.

\title{
EXISTENCE AND STABILITY ANALYSIS OF SEQUENTIAL COUPLED SYSTEM OF HADAMARD-TYPE FRACTIONAL DIFFERENTIAL EQUATIONS
}

\author{
AKBAR ZADA ${ }^{1}$ AND MOHAMMAD YAR ${ }^{1}$
}

\begin{abstract}
In this paper we study existence, uniqueness and Hyers-Ulam stability for a sequential coupled system consisting of fractional differential equations of Hadamard type, subject to nonlocal Hadamard fractional integral boundary conditions. The existence of solutions is derived from Leray-Schauder's alternative, whereas the uniqueness of solution is established by Banach contraction principle. An example is also presented which illustrate our results.
\end{abstract}

\section{INTRODUCTION}

In this paper, we study the sequential coupled system of Hadamard fractional differential equations with nonlocal Hadamard fractional integral boundary conditions of the following form:

$$
\left\{\begin{array}{l}
\left(D^{q}+k D^{q-1}\right) u(t)=f(t, u(t), v(t)), \quad k>0,1<q \leq 2, t \in(1, e), \\
\left(D^{p}+k D^{p-1}\right) v(t)=g(t, u(t), v(t)), \quad k>0,1<p \leq 2, t \in(1, e), \\
u(1)=0, \quad \sum_{i=1}^{m} \lambda_{i} I^{\alpha_{i}} u\left(\eta_{i}\right)=\sum_{j=1}^{n} \mu_{j}\left(I^{\beta_{j}} u(e)-I^{\beta_{j}} u\left(\xi_{j}\right)\right), \\
v(1)=0, \quad \sum_{i=1}^{m} \rho_{i} I^{\gamma_{i}} v\left(\theta_{i}\right)=\sum_{j=1}^{n} \kappa_{j}\left(I^{\delta_{j}} v(e)-I^{\delta_{j}} v\left(\zeta_{j}\right)\right)
\end{array}\right.
$$

where $D^{(\cdot)}$ denotes the Hadamard fractional derivative of order $p$ and $q, f, g:[1, e] \times$ $\mathbb{R}^{2} \rightarrow \mathbb{R}$ are continuous functions and $\eta_{i}, \theta_{i}, \xi_{j}, \zeta_{j} \in(1, e)$ and $\lambda_{i}, \rho_{i}, \mu_{j}, \kappa_{j} \in \mathbb{R}$,

Key words and phrases. Hadamard fractional derivative, sequential coupled system, fixed point theorem, Hyers-Ulam stability.

2010 Mathematics Subject Classification. Primary: 26A33. Secondary: 34A08, 35B40.

DOI 10.46793/KgJMat2201.085Z

Received: April 07, 2019.

Accepted: September 01, 2019. 
$i=1,2, \ldots, m, j=1,2, \ldots, n$, and $I^{(\phi)}$ is the Hadamard fractional integral of order $\phi>0, \phi=\alpha_{i}, \gamma_{i}, \beta_{j}, \delta_{j}, i=1,2, \ldots, m, j=1,2, \ldots, n$.

Fractional calculus is the field of mathematical analysis, which deals with the investigation and applications of integrals and derivatives of an arbitrary order. Fractional differential equations (FDEs) have played a significant role in many engineering and scientific disciplines e.g. as the mathematical modeling of systems and processes in the fields of physics, chemistry, aerodynamics, electrodynamics of complex medium, polymer rheology, bode's analysis of feedback amplifiers, capacitor theory, electrical circuits, electron analytical chemistry, biology, control theory, fitting of experimental data and so forth $[5,14,15]$. FDEs also serve as an excellent tool for the description of hereditary properties of various materials and processes [18].

The theory of fractional order differential equations, involving different kinds of boundary conditions has been a field of interest in pure and applied sciences. Nonlocal conditions are used to describe certain features of applied mathematics and physics such as blood flow problems, chemical engineering, thermo-elasticity, underground water flow, population dynamics and so on $[1,6,17]$.

In the classical text [19], it has been mentioned that Hadamard in 1892 [8] suggested a concept of fractional integro-differentiation in terms of the fractional power of the type $\left(x \frac{d}{d x}\right)^{p}$ in contrast to its Riemann-Liouville counterpart of the form $\left(\frac{d}{d x}\right)^{p}$. The kind of derivative, introduced by Hadamard contains logarithmic function of arbitrary exponent in the kernel of the integral appearing in its definition. Hadamard's construction is invariant in relation to dilation and is well suited to the problems containing half axes.

Coupled systems of FDEs have also been investigated by many authors. Such systems appear naturally in many real world situations. Some recent results on the topic can be found in a series of papers $[2,3,9,12,20,25]$.

Another aspect of FDEs which has very recently got attentions from the researchers is the Ulam type stability analysis of the aforesaid equations. The mentioned stability was first pointed out by Ulam [26] in 1940, which was further explained by Hyers [10], over Banach space. Latter on, many researchers done valuable work on the same task and interesting results were formed for linear and nonlinear integral and differential equations, for detail see $[4,24,27,28,30-32,35]$. This stability analysis is very useful in many applications, such as numerical analysis, optimization, etc., where finding the exact solution is quite difficult. For detailed study of Ulam-type stability with different approaches, we recommend papers [13, 21-24, 29, 33, 34, 36-39].

In addition, the inspiration of this paper comes from the following two problems $[11,16]$. In [16], Thiramanus et al. investigated the existence and uniqueness of solutions for a fractional boundary value problem involving Hadamard-type fractional 
differential equations and nonlocal fractional integral boundary conditions:

$$
\left\{\begin{array}{l}
D^{q} x(t)=f(t, x(t)), \quad 1<q \leq 2, t \in(1, e), \\
x(1)=0 \\
\sum_{i=1}^{m} \lambda_{i} I^{\alpha_{i}} x\left(\eta_{i}\right)=\sum_{j=1}^{n} \mu_{j}\left(I^{\beta_{j}} x(e)-I^{\beta_{j}} x\left(\xi_{j}\right)\right)
\end{array}\right.
$$

where $D^{q}$ denotes the Hadamard fractional derivative of order $\mathrm{q}, f:[1, e] \times \mathbb{R} \rightarrow \mathbb{R}$ is a continuous function, $\eta_{i}, \xi_{j} \in(1, e), \lambda_{i}, \mu_{j} \in \mathbb{R}$ for all $i=1,2, \ldots, m, j=1,2, \cdots, n$, $\eta_{1}<\eta_{2}<\cdots<\eta_{m}, \xi_{1}<\xi_{2}<\cdots<\xi_{n}$ and $I \phi$ is the Hadamard fractional integral of order $\phi>0, \phi=\alpha_{i}, \beta_{j}, i=1,2, \ldots, m, j=1,2, \ldots, n$.

In [11], the authors discussed existence results for Hadamard type fractional functional integro-differential equations with integral boundary conditions:

$$
\left\{\begin{array}{l}
D^{q} y(t)=f\left(t, y(t),\left(T_{1} y\right)(t),\left(T_{2} y\right)(t)\right), \quad 1<q \leq 2, t \in(1, e) \\
y(1)=0 \\
\sum_{i=1}^{m} \lambda_{i} I^{\alpha_{i}} y\left(\eta_{i}\right)=\sum_{j=1}^{n} \mu_{j}\left(I^{\beta_{j}} x(e)-I^{\beta_{j}} x\left(\xi_{j}\right)\right) .
\end{array}\right.
$$

We show the existence of solutions for problem (1.1) by applying Leray-Schauder alternative criterion while uniqueness of solutions for (1.1) relies on Banach contraction mapping principle.

The rest of the paper is organized as follows: In Section 2, we recall some preliminary concepts which we need in the sequel. Section 3 contains the main results for problem (1.1). In Section 4, we present the Hyers-Ulam stability for problem (1.1).

\section{Preliminaries and Background Materials}

In this section, we introduce some notations and definitions of fractional calculus and present preliminary results needed in our proofs, and then we prove an auxiliary lemma for the linear modification of problem (1.1).

Definition 2.1. The Hadamard derivative of fractional order $q$ for a function $f \in$ $C^{n}[1, \infty)$, is defined as

$$
D^{q} f(t)=\frac{1}{\Gamma(n-q)}\left(t \frac{d}{d t}\right)^{n} \int_{0}^{t}\left(\log \frac{t}{s}\right)^{n-q-1} \frac{f(s)}{s} d s, \quad n-1<q<n,
$$

where $n=[q]+1,[q]$ denotes the integer part of $q$ and $\log (\cdot)=\log _{e}(\cdot)$ provided that integral exists.

Definition 2.2. The Hadamard fractional integral of order $q$ for a function $f$ : $[1, \infty) \rightarrow \mathbb{R}$ is defined by

$$
I^{q} f(t)=\frac{1}{\Gamma(q)} \int_{1}^{t}\left(\log \frac{t}{s}\right)^{q-1} \frac{f(s)}{s} d s, \quad q>0
$$


provided that integral exists, and $\Gamma(\cdot)$ is the Gamma function defined by

$$
\Gamma(q)=\int_{0}^{\infty} e^{-s} t^{q-1} d s, \quad q>0 .
$$

Lemma 2.1. Let $\phi, \psi$ are continuous functions from $[1, e]$ to $\mathbb{R}$. Then the solution for the linear system of sequential fractional differential equations:

$$
\left\{\begin{array}{l}
\left(D^{q}+k D^{q-1}\right) u(t)=\phi(t), \quad k>0,1<q \leq 2, t \in(1, e), \\
\left(D^{p}+k D^{p-1}\right) v(t)=\psi(t), \quad 1<p \leq 2, t \in(1, e), \\
u(1)=0, \quad \sum_{i=1}^{m} \lambda_{i} I^{\alpha_{i}} u\left(\eta_{i}\right)=\sum_{j=1}^{n} \mu_{j}\left(I^{\beta_{j}} u(e)-I^{\beta_{j}} u\left(\xi_{j}\right)\right), \\
v(1)=0, \quad \sum_{i=1}^{m} \rho_{i} I^{\gamma_{i}} v\left(\theta_{i}\right)=\sum_{j=1}^{n} \kappa_{j}\left(I^{\delta_{j}} v(e)-I^{\delta_{j}} v\left(\zeta_{j}\right)\right),
\end{array}\right.
$$

is

$$
\begin{aligned}
u(t)= & \frac{1}{A_{1}}\left(t^{-k} \int_{1}^{t} s^{k-1}(\log s)^{q-2} d s\right)\left[\sum_{i=1}^{m} \frac{\lambda_{i}}{\Gamma\left(\alpha_{i}\right) \Gamma(q-1)}\right. \\
& \times \int_{1}^{\eta_{i}}\left(\log \frac{\eta_{i}}{s}\right)^{\alpha_{i}-1} s^{-k-1}\left(\int_{1}^{s} r^{k-1}\left(\int_{1}^{r}\left(\log \frac{r}{m}\right)^{q-2} \frac{\phi(m)}{m} d m\right) d r\right) d s \\
& +\sum_{j=1}^{n} \frac{\mu_{j}}{\Gamma\left(\beta_{j}\right) \Gamma(q-1)} \int_{1}^{\xi_{j}}\left(\log \frac{\xi_{j}}{s}\right)^{\beta_{j}-1} s^{-k-1} \\
& \times\left(\int_{1}^{s} r^{k-1}\left(\int_{1}^{r}\left(\log \frac{r}{m}\right)^{q-2} \frac{\phi(m)}{m} d m\right) d r\right) d s \\
& -\frac{e^{-k}}{\Gamma(q-1)} \sum_{j=1}^{n} \frac{\mu_{j}}{\Gamma\left(\beta_{j}\right)} \int_{1}^{e}\left(\log \frac{e}{s}\right)^{\beta_{j}-1} s^{-1} \\
& \left.\times\left(\int_{1}^{e} r^{k-1}\left(\int_{1}^{r}\left(\log \frac{r}{m}\right)^{q-2} \frac{\phi(m)}{m} d m\right) d r\right) d s\right] \\
& +t^{-k} \int_{1}^{t} s^{k-1} I^{q-1} \phi(s) d s
\end{aligned}
$$

and

$$
\begin{aligned}
v(t)= & \frac{1}{A_{2}}\left(t^{-k} \int_{1}^{t} s^{k-1}(\log s)^{p-2} d s\right)\left[\sum_{i=1}^{m} \frac{\rho_{i}}{\Gamma\left(\gamma_{i}\right) \Gamma(p-1)}\right. \\
& \times \int_{1}^{\theta_{i}}\left(\log \frac{\theta_{i}}{s}\right)^{\gamma_{i}-1} s^{-k-1}\left(\int_{1}^{s} r^{k-1}\left(\int_{1}^{r}\left(\log \frac{r}{m}\right)^{p-2} \frac{\psi(m)}{m} d m\right) d r\right) d s \\
& +\sum_{j=1}^{n} \frac{\kappa_{j}}{\Gamma\left(\delta_{j}\right) \Gamma(p-1)} \int_{1}^{\zeta_{j}}\left(\log \frac{\zeta_{j}}{s}\right)^{\delta_{j}-1} s^{-k-1} \\
& \times\left(\int_{1}^{s} r^{k-1}\left(\int_{1}^{r}\left(\log \frac{r}{m}\right)^{p-2} \frac{\psi(m)}{m} d m\right) d r\right) d s
\end{aligned}
$$




$$
\begin{aligned}
& -\frac{e^{-k}}{\Gamma(p-1)} \sum_{j=1}^{n} \frac{\kappa_{j}}{\Gamma\left(\delta_{j}\right)} \int_{1}^{e}\left(\log \frac{e}{s}\right)^{\delta_{j}-1} s^{-1} \\
& \left.\times\left(\int_{1}^{e} r^{k-1}\left(\int_{1}^{r}\left(\log \frac{r}{m}\right)^{p-2} \frac{\psi(m)}{m} d m\right) d r\right) d s\right] \\
& +t^{-k} \int_{1}^{t} s^{k-1} I^{p-1} \psi(s) d s
\end{aligned}
$$

where

$$
\begin{aligned}
A_{1}= & \sum_{j=1}^{n} \frac{e^{-k} \mu_{j}}{\Gamma\left(\beta_{j}\right)} \int_{1}^{e}\left(\log \frac{e}{s}\right)^{\beta_{j}-1} s^{-1}\left(\int_{1}^{e} r^{k-1}(\log r)^{q-2} d r\right) d s \\
& -\sum_{j=1}^{n} \frac{\mu_{j}}{\Gamma\left(\beta_{j}\right)} \int_{1}^{\xi_{j}}\left(\log \frac{\xi_{j}}{s}\right)^{\beta_{j}-1} s^{-k-1}\left(\int_{1}^{r} r^{k-1}(\log r)^{q-2} d r\right) d s \\
& -\sum_{i=1}^{m} \frac{\lambda_{i}}{\Gamma\left(\alpha_{i}\right)} \int_{1}^{\eta_{i}}\left(\log \frac{\eta_{i}}{s}\right)^{\alpha_{i}-1} s^{-k-1}\left(\int_{1}^{r} r^{k-1}(\log r)^{q-2} d r\right) d s
\end{aligned}
$$

and

$$
\begin{aligned}
A_{2}= & \sum_{j=1}^{n} \frac{e^{-k} \kappa_{j}}{\Gamma\left(\delta_{j}\right)} \int_{1}^{e}\left(\log \frac{e}{s}\right)^{\delta_{j}-1} s^{-1}\left(\int_{1}^{e} r^{k-1}(\log r)^{p-2} d r\right) d s \\
& -\sum_{j=1}^{n} \frac{\kappa_{j}}{\Gamma\left(\delta_{j}\right)} \int_{1}^{\zeta_{j}}\left(\log \frac{\zeta_{j}}{s}\right)^{\delta_{j}-1} s^{-k-1}\left(\int_{1}^{r} r^{k-1}(\log r)^{p-2} d r\right) d s \\
& -\sum_{i=1}^{m} \frac{\rho_{i}}{\Gamma\left(\gamma_{i}\right)} \int_{1}^{\theta_{i}}\left(\log \frac{\theta_{i}}{s}\right)^{\gamma_{i}-1} s^{-k-1}\left(\int_{1}^{r} r^{k-1}(\log r)^{p-2} d r\right) d s .
\end{aligned}
$$

Proof. As argued in [14], the general solution of the system (2.1) can be written as

$$
u(t)=c_{0} t^{-k}+c_{1} t^{-k} \int_{1}^{t} s^{k-1}(\log s)^{q-2} d s+t^{-k} \int_{1}^{t} s^{k-1} I^{q-1} \phi(t) d s
$$

and

$$
v(t)=d_{0} t^{-k}+d_{1} t^{-k} \int_{1}^{t} s^{k-1}(\log s)^{p-2} d s+t^{-k} \int_{1}^{t} s^{k-1} I^{p-1} \psi(t) d s,
$$

where $c_{i}, d_{i}, i=0,1$, are unknown arbitrary constants. The conditions $u(1)=0$ and $v(1)=0$ in (2.6) and (2.7) imply that $c_{0}=0$ and $d_{0}=0$, which leads to

$$
u(t)=c_{1} t^{-k} \int_{1}^{t} s^{k-1}(\log s)^{q-2} d s+t^{-k} \int_{1}^{t} s^{k-1} I^{q-1} \phi(t) d s
$$

and

$$
v(t)=d_{1} t^{-k} \int_{1}^{t} s^{k-1}(\log s)^{p-2} d s+t^{-k} \int_{1}^{t} s^{k-1} I^{p-1} \psi(t) d s .
$$


Now, using the coupled integral boundary conditions given by (1.1), in (2.8) and (2.9), we obtain

$$
c_{1}=\frac{J_{1}}{A_{1}}, \quad d_{1}=\frac{J_{2}}{A_{2}},
$$

where $A_{1}$ and $A_{2}$ are respectively given by (2.4) and (2.5), and

$$
\begin{aligned}
J_{1}= & \sum_{i=1}^{m} \frac{\lambda_{i}}{\Gamma\left(\alpha_{i}\right) \Gamma(q-1)} \int_{1}^{\eta_{i}}\left(\log \frac{\eta_{i}}{s}\right)^{\alpha_{i}-1} s^{-k-1} \\
& \times\left(\int_{1}^{s} r^{k-1}\left(\int_{1}^{r}\left(\log \frac{r}{m}\right)^{q-2} \frac{\phi(m)}{m} d m\right) d r\right) d s \\
& +\sum_{j=1}^{n} \frac{\mu_{j}}{\Gamma\left(\beta_{j}\right) \Gamma(q-1)} \int_{1}^{\xi_{j}}\left(\log \frac{\xi_{j}}{s}\right)^{\beta_{j}-1} s^{-k-1} \\
& \times\left(\int_{1}^{s} r^{k-1}\left(\int_{1}^{r}\left(\log \frac{r}{m}\right)^{q-2} \frac{\phi(m)}{m} d m\right) d r\right) d s \\
& -\sum_{j=1}^{n} \frac{e^{-k} \mu_{j}}{\Gamma\left(\beta_{j}\right)} \int_{1}^{e}\left(\log \frac{e}{s}\right)^{\beta_{j}-1} s^{-1}\left(\int_{1}^{e} r^{k-1} I^{q-1} \phi(r) d r\right) d s \\
J_{2}= & \sum_{i=1}^{m} \frac{\rho_{i}}{\Gamma\left(\gamma_{i}\right) \Gamma(p-1)} \int_{1}^{\theta_{i}}\left(\log \frac{\theta_{i}}{s}\right)^{\gamma_{i}-1} s^{-k-1} \\
& \times\left(\int_{1}^{s} r^{k-1}\left(\int_{1}^{r}\left(\log \frac{r}{m}\right)^{p-2} \frac{\psi(m)}{m} d m\right) d r\right) d s \\
& +\sum_{j=1}^{n} \frac{\kappa_{j}}{\Gamma\left(\delta_{j}\right) \Gamma(p-1)} \int_{1}^{\zeta_{j}}\left(\log \frac{\zeta_{j}}{s}\right)^{\delta_{j}-1} s^{-k-1} \\
& \times\left(\int_{1}^{s} r^{k-1}\left(\int_{1}^{r}\left(\log \frac{r}{m}\right)^{p-2} \frac{\psi(m)}{m} d m\right) d r\right) d s \\
& -\sum_{j=1}^{n} \frac{e^{-k} \kappa_{j}}{\Gamma\left(\delta_{j}\right)} \int_{1}^{e}\left(\log \frac{e}{s}\right)^{\delta_{j}-1} s^{-1}\left(\int_{1}^{e} r^{k-1} I^{p-1} \psi(r) d r\right) d s .
\end{aligned}
$$

Substituting the values of $c_{1}$ and $c_{2}$ in (2.8) and (2.9) we obtained the solutions (2.2) and (2.3).

\section{MAin Results}

Let $\mathcal{C}=C([1, e], \mathbb{R})$ denotes the Banach space of all continuous functions from $[1, e]$ to $\mathbb{R}$. Let us introduce the space $X=\left\{u(t): u(t) \in C^{1}([1, e])\right\}$ endowed with the norm $\|u\|=\sup \{|u(t)|: t \in[1, e]\}$. Obviously, $(X,\|\cdot\|)$ is a Banach space. Also let $Y=\left\{v(t): v(t) \in C^{1}([1, e])\right\}$ be endowed with the norm $\|v\|=\sup \{|v(t)|: t \in[1, e]\}$. Obviously the product space $(X \times Y,\|(u, v)\|)$ is a Banach space with norm $\|(u, v)\|=$ 
$\|u\|+\|v\|$. In view of Lemma 2.1, we define an operator $\mathcal{T}: X \times Y \rightarrow X \times Y$ by

$$
\mathcal{T}(u, v)(t)=\left(\begin{array}{c}
\mathcal{T}_{1}(u, v)(t) \\
\mathcal{T}_{2}(u, v)(t)
\end{array}\right)
$$

where

$$
\begin{aligned}
\mathcal{T}_{1}(u, v)(t)= & \frac{1}{A_{1}}\left(t^{-k} \int_{1}^{t} s^{k-1}(\log s)^{q-2} d s\right) \\
& {\left[\sum_{i=1}^{m} \frac{\lambda_{i}}{\Gamma\left(\alpha_{i}\right)} \int_{1}^{\eta_{i}}\left(\log \frac{\eta_{i}}{s}\right)^{\alpha_{i}-1} s^{-k-1}\left(\int_{1}^{s} r^{k-1}\left(I^{q-1} f(r, u(r), v(r))\right) d r\right) d s\right.} \\
& +\sum_{j=1}^{n} \frac{\mu_{j}}{\Gamma\left(\beta_{j}\right)} \int_{1}^{\xi_{j}}\left(\log \frac{\xi_{j}}{s}\right)^{\beta_{j}-1} s^{-k-1}\left(\int_{1}^{s} r^{k-1}\left(I^{q-1} f(r, u(r), v(r))\right) d r\right) d s \\
& \left.-\sum_{j=1}^{n} \frac{e^{-k} \mu_{j}}{\Gamma\left(\beta_{j}\right)} \int_{1}^{e}\left(\log \frac{e}{s}\right)^{\beta_{j}-1} s^{-1}\left(\int_{1}^{e} r^{k-1} I^{q-1} f(r, u(r), v(r)) d r\right) d s\right] \\
& +t^{-k} \int_{1}^{t} s^{k-1} I^{q-1} f(s, u(s), v(s)) d s
\end{aligned}
$$

and

$$
\begin{aligned}
\mathcal{T}_{2}(u, v)(t)= & \frac{1}{A_{2}}\left(t^{-k} \int_{1}^{t} s^{k-1}(\log s)^{p-2} d s\right) \\
& {\left[\sum_{i=1}^{m} \frac{\rho_{i}}{\Gamma\left(\gamma_{i}\right)} \int_{1}^{\theta_{i}}\left(\log \frac{\theta_{i}}{s}\right)^{\gamma_{i}-1} s^{-k-1}\left(\int_{1}^{s} r^{k-1}\left(I^{p-1} g(r, u(r), v(r))\right) d r\right) d s\right.} \\
& +\sum_{j=1}^{n} \frac{\kappa_{j}}{\Gamma\left(\delta_{j}\right)} \int_{1}^{\zeta_{j}}\left(\log \frac{\zeta_{j}}{s}\right)^{\delta_{j}-1} s^{-k-1}\left(\int_{1}^{s} r^{k-1}\left(I^{p-1} g(r, u(r), v(r))\right) d r\right) d s \\
& \left.-\sum_{j=1}^{n} \frac{e^{-k} \kappa_{j}}{\Gamma\left(\delta_{j}\right)} \int_{1}^{e}\left(\log \frac{e}{s}\right)^{\delta_{j}-1} s^{-1}\left(\int_{1}^{e} r^{k-1} I^{p-1} g(r, u(r), v(r)) d r\right) d s\right] \\
& +t^{-k} \int_{1}^{t} s^{k-1} I^{p-1} g(s, u(s), v(s)) d s .
\end{aligned}
$$

For the sake of convenience, we set

$$
\begin{aligned}
M_{1}= & \frac{1}{\left|A_{1}\right|}\left(\sum_{i=1}^{m} \frac{\left|\lambda_{i}\right|\left(\log \eta_{i}\right)^{\alpha_{i}+q}}{(q-1) \Gamma(q+1) \Gamma\left(\alpha_{i}+1\right)}+\sum_{j=1}^{n} \frac{\left|\mu_{j}\right|\left(\log \xi_{j}\right)^{\beta_{j}+q}}{(q-1) \Gamma(q+1) \Gamma\left(\beta_{j}+1\right)}\right) \\
& +\sum_{j=1}^{n} \frac{\left|\mu_{j}\right|}{(q-1) \Gamma(q+1) \Gamma\left(\beta_{j}+1\right)}, \\
M_{2}= & \frac{1}{\left|A_{2}\right|}\left(\sum_{i=1}^{m} \frac{\left|\rho_{i}\right|\left(\log \theta_{i}\right)^{\gamma_{i}+p}}{(p-1) \Gamma(p+1) \Gamma\left(\gamma_{i}+1\right)}+\sum_{j=1}^{n} \frac{\left|\kappa_{j}\right|\left(\log \zeta_{j}\right)^{\delta_{j}+p}}{(p-1) \Gamma(p+1) \Gamma\left(\delta_{j}+1\right)}\right)
\end{aligned}
$$




$$
+\sum_{j=1}^{n} \frac{\left|\kappa_{j}\right|}{(p-1) \Gamma(p+1) \Gamma\left(\delta_{j}+1\right)}
$$

and

$$
M_{0}=\min \left\{1-\left(M_{1} k_{1}+M_{2} \lambda_{1}\right), 1-\left(M_{1} k_{2}+M_{2} \lambda_{2}\right\}, \quad k_{i}, \lambda_{i} \leq 0, i=1,2 .\right.
$$

The first result is concerned with the existence and uniqueness of solution for the problem (1.1) and is based on Banach contraction mapping principle.

Theorem 3.1. Assume that $f, g:[1, e] \times \mathbb{R}^{\not} \rightarrow \mathbb{R}$ are continuous functions and there exist constants $m_{i}, n_{i}, i=1,2$ such that for all $t \in[1, e]$ and $u_{i}, v_{i} \in \mathbb{R}, i=1,2$, such that

$$
\left|f\left(t, u_{2}, v_{2}\right)-f\left(t, u_{1}, v_{1}\right)\right| \leq m_{1}\left|u_{2}-u_{1}\right|+m_{2}\left|v_{2}-v_{1}\right|
$$

and

$$
\left|g\left(t, u_{2}, v_{2}\right)-g\left(t, u_{1}, v_{1}\right)\right| \leq n_{1}\left|u_{2}-u_{1}\right|+n_{2}\left|v_{2}-v_{1}\right| .
$$

In addition, assume that

$$
M_{1}\left(m_{1}+m_{2}\right)+M_{2}\left(\left(n_{1}+n_{2}\right)<1,\right.
$$

where $M_{i}, i=1,2$, are given by (3.3) and (3.4). Then the boundary value problem (1.1) has a unique solution.

Proof. Define $\sup _{t \in[1, e]} f(t, 0,0)=N_{1}<\infty$ and $\sup _{t \in[1, e]} g(t, 0,0)=N_{2}<\infty$ such that

$$
r>\frac{M_{1} N_{1}+M_{2} N_{2}}{1-M_{1}\left(m_{1}+m_{2}\right)+M_{2}\left(n_{1}+n_{2}\right)} .
$$

We show that $\mathcal{T} \mathbf{B}_{\mathbf{r}} \subset \mathbf{B}_{\mathbf{r}}$, where $\mathbf{B}_{\mathbf{r}}=\{(u, v) \in X \times Y:\|(u, v)\|<r\}$. For $(u, v) \in \mathbf{B}_{\mathbf{r}}$, we have

$$
\begin{aligned}
\left|\mathcal{T}_{1}(u, v)(t)\right|= & \sup _{t \in[1, e]}\left\{\frac { 1 } { A _ { 1 } } ( t ^ { - k } \int _ { 1 } ^ { t } s ^ { k - 1 } ( \operatorname { l o g } s ) ^ { q - 2 } d s ) \left[\sum_{i=1}^{m} \frac{\lambda_{i}}{\Gamma\left(\alpha_{i}\right) \Gamma(q-1)}\right.\right. \\
& \times \int_{1}^{\eta_{i}}\left(\log \frac{\eta_{i}}{s}\right)^{\alpha_{i}-1} s^{-k-1}\left(\int _ { 1 } ^ { s } r ^ { k - 1 } \left(\int_{1}^{r}\left(\log \frac{r}{m}\right)^{q-2}\right.\right. \\
& \left.\left.\times \frac{f(m, u(m), v(m))}{m} d m\right) d r\right) d s+\sum_{j=1}^{n} \frac{\mu_{j}}{\Gamma\left(\beta_{j}\right) \Gamma(q-1)} \\
& \times \int_{1}^{\xi_{j}}\left(\log \frac{\xi_{j}}{s}\right)^{\beta_{j}-1} s^{-k-1}\left(\int _ { 1 } ^ { s } r ^ { k - 1 } \left(\int_{1}^{r}\left(\log \frac{r}{m}\right)^{q-2}\right.\right. \\
& \left.\left.\frac{f(m, u(m), v(m))}{m} d m\right) d r\right) d s-\sum_{j=1}^{n} \frac{e^{-k} \mu_{j}}{\Gamma\left(\beta_{j}\right) \Gamma(q-1)} \int_{1}^{e}\left(\log \frac{e}{s}\right)^{\beta_{j}-1} s^{-1} \\
& \left.\times\left(\int_{1}^{e} r^{k-1}\left(\int_{1}^{r}\left(\log \frac{r}{m}\right)^{q-2} \frac{f(m, u(m), v(m))}{m} d m\right) d r\right) d s\right]
\end{aligned}
$$




$$
\begin{aligned}
& \left.+\frac{t^{-k}}{\Gamma(q-1)} \int_{1}^{t} s^{k-1}\left(\int_{1}^{s}\left(\log \frac{s}{r}\right)^{q-2} \frac{f(r, u(r), v(r))}{r} d r\right) d s\right\} \\
& \leq \frac{1}{\left|A_{1}\right|}\left(\int_{1}^{t} s^{k-1}(\log s)^{q-2} d s\right)\left[\sum_{i=1}^{m} \frac{\left|\lambda_{i}\right|}{\Gamma\left(\alpha_{i}\right) \Gamma(q-1)} \int_{1}^{\eta_{i}}\left(\log \frac{\eta_{i}}{s}\right)^{\alpha_{i}-1} s^{-k-1}\right. \\
& \times\left(\int _ { 1 } ^ { s } r ^ { k - 1 } \left(\int_{1}^{r}\left(\log \frac{r}{m}\right)^{q-2}\right.\right. \\
& \left.\left.\times \frac{(|f(m, u(m), v(m))-f(m, 0,0)|+|f(m, 0,0)|)}{m} d m\right) d r\right) d s \\
& +\sum_{j=1}^{n} \frac{\left|\mu_{j}\right|}{\Gamma\left(\beta_{j}\right) \Gamma(q-1)} \int_{1}^{\xi_{j}}\left(\log \frac{\xi_{j}}{s}\right)^{\beta_{j}-1} s^{-k-1}\left(\int _ { 1 } ^ { s } r ^ { k - 1 } \left(\int_{1}^{r}\left(\log \frac{r}{m}\right)^{q-2}\right.\right. \\
& \left.\left.\times \frac{(|f(m, u(m), v(m))-f(m, 0,0)|+|f(m, 0,0)|)}{m} d m\right) d r\right) d s \\
& +\sum_{j=1}^{n} \frac{\left|\mu_{j}\right|}{\Gamma\left(\beta_{j}\right) \Gamma(q-1)} \int_{1}^{e}\left(\log \frac{e}{s}\right)^{\beta_{j}-1} s^{-1}\left(\int _ { 1 } ^ { e } r ^ { k - 1 } \left(\int_{1}^{r}\left(\log \frac{r}{m}\right)^{q-2}\right.\right. \\
& \left.\left.\left.\times \frac{(|f(m, u(m), v(m))-f(m, 0,0)|+|f(m, 0,0)|)}{m} d m\right) d r\right) d s\right] \\
& +\frac{1}{\Gamma(q-1)} \int_{1}^{t} s^{k-1}\left(\int_{1}^{s}\left(\log \frac{s}{r}\right)^{q-2}\right. \\
& \left.\times \frac{(|f(r, u(r), v(r))-f(r, 0,0)|+|f(r, 0,0)|)}{r} d r\right) d s \\
& \leq\left(m_{1}\|u\|+m_{2}\|v\|+N_{1}\right) \\
& \times\left[\frac { 1 } { | A _ { 1 } | } \left(\sum_{i=1}^{m} \frac{\left|\lambda_{i}\right|\left(\log \eta_{i}\right)^{\alpha_{i}+q}}{(q-1) \Gamma\left(\alpha_{i}+1\right) \Gamma(q+1)}\right.\right. \\
& \left.+\sum_{j=1}^{n} \frac{\left|\mu_{j}\right|\left(\log \xi_{j}\right)^{\beta_{j}+q}}{(q-1) \Gamma\left(\beta_{j}+1\right) \Gamma(q+1)}\right) \\
& \left.+\sum_{j=1}^{n} \frac{\left|\mu_{j}\right|}{(q-1) \Gamma\left(\beta_{j}+1\right) \Gamma(q+1)}\right] \\
& =M_{1}\left[m_{1}\|u\|+m_{2}\|v\|+N_{1}\right] \\
& =M_{1}\left[\left(m_{1}+m_{2}\right) r+N_{1}\right] \text {. }
\end{aligned}
$$

Hence,

$$
\left|\mathcal{T}_{1}(u, v)(t)\right| \leq M_{1}\left[\left(m_{1}+m_{2}\right) r+N_{1}\right] .
$$

In the same way, we can obtain that

$$
\left|\mathcal{T}_{2}(u, v)(t)\right| \leq M_{2}\left[\left(n_{1}+n_{2}\right) r+N_{2}\right]
$$


Consequently, $|\mathcal{T}(u, v)(t)| \leq r$. Now, for $\left(u_{1}, v_{1}\right),\left(u_{2}, v_{2}\right) \in X \times Y$, and for any $t \in[1, e]$, we get

$$
\begin{aligned}
& \left|\mathcal{T}_{1}\left(u_{2}, v_{2}\right)(t)-\mathcal{T}_{1}\left(u_{1}, v_{1}\right)(t)\right| \\
\leq & \frac{1}{\left|A_{1}\right|}\left(t^{-k} \int_{1}^{t} s^{k-1}(\log s)^{q-2} d s\right)\left[\sum_{i=1}^{m} \frac{\left|\lambda_{i}\right|}{\Gamma\left(\alpha_{i}\right) \Gamma(q-1)} \int_{1}^{\eta_{i}}\left(\log \frac{\eta_{i}}{s}\right)^{\alpha_{i}-1} s^{-k-1}\right. \\
& \times\left(\int _ { 1 } ^ { s } r ^ { k - 1 } \left(\int_{1}^{r}\left(\log \frac{r}{m}\right)^{q-2}\right.\right. \\
& \left.\left.\frac{\left|f\left(m, u_{2}(m), v_{2}(m)\right)-f\left(m, u_{1}(m), v_{1}(m)\right)\right|}{m} d m\right) d r\right) d s \\
& +\sum_{j=1}^{n} \frac{\left|\mu_{j}\right|}{\Gamma\left(\beta_{j}\right) \Gamma(q-1)} \int_{1}^{\xi_{j}}\left(\log \frac{\xi_{j}}{s}\right)^{\beta_{j}-1} s^{-k-1} \\
& \times\left(\int _ { 1 } ^ { s } r ^ { k - 1 } \left(\int_{1}^{r}\left(\log \frac{r}{m}\right)^{q-2}\right.\right. \\
& \left.\left.\frac{\left|f\left(m, u_{2}(m), v_{2}(m)\right)-f\left(m, u_{1}(m), v_{1}(m)\right)\right|}{m} d m\right) d r\right) d s \\
& \sum_{j=1}^{n} \frac{\left|\mu_{j}\right|}{\Gamma\left(\beta_{j}\right) \Gamma(q-1)} \int_{1}^{e}\left(\log \frac{e}{s}\right)^{\beta_{j}-1} s^{-1} \\
& \times\left(M _ { 1 } ^ { e } r ^ { k - 1 } \left(\int_{1}^{r}\left(\log \frac{r}{m}\right)^{q-2}\right.\right. \\
\leq & M_{1}\left(m_{1}+m_{2}\right)\left(\left\|u_{2}-u_{1}\right\|+\left\|v_{2}-v_{1}\right\|\right), \\
& \frac{\left|f\left(r, u_{2}(r), v_{2}(r)\right)-f\left(r, u_{1}(r), v_{1}(r)\right)\right|}{\Gamma(q-1)} \int_{1}^{t} s^{k-1}\left(\int_{1}^{s}\left(\log \frac{s}{r}\right)^{q-2} d s\right. \\
& \left.\left.\left.\frac{f\left(m, u_{2}(m), v_{2}(m)\right)-f\left(m, u_{1}(m), v_{1}(m)\right) \mid}{m} d m\right) d r\right) d s\right]
\end{aligned}
$$

and consequently, we obtain

(3.6) $\left|\mathcal{T}_{1}\left(u_{2}, v_{2}\right)(t)-\mathcal{T}_{1}\left(u_{1}, v_{1}\right)(t)\right| \leq M_{1}\left(m_{1}+m_{2}\right)\left(\left\|u_{2}-u_{1}\right\|+\left\|v_{2}-v_{1}\right\|\right)$.

Similarly,

$$
\left|\mathcal{T}_{2}\left(u_{2}, v_{2}\right)(t)-\mathcal{T}_{2}\left(u_{1}, v_{1}\right)(t)\right| \leq M_{2}\left(n_{1}+n_{2}\right)\left(\left\|u_{2}-u_{1}\right\|+\left\|v_{2}-v_{1}\right\|\right) .
$$

It follows from (3.6) and (3.7) that

$$
\left|\mathcal{T}\left(u_{2}, v_{2}\right)(t)-\mathcal{T}\left(u_{1}, v_{1}\right)(t)\right| \leq\left[M_{1}\left(m_{1}+m_{2}\right)+M_{2}\left(n_{1}+n_{2}\right)\right]
$$




$$
\times\left(\left\|u_{2}-u_{1}\right\|+\left\|v_{2}-v_{1}\right\|\right) .
$$

Since $M_{1}\left(m_{1}+m_{2}\right)+M_{2}\left(n_{1}+n_{2}\right)<1$, therefore, $\mathcal{T}$ is a contraction operator. So, by Banach fixed point theorem, the operator $\mathcal{T}$ has a unique fixed point, which is the unique solution of problem (1.1). This completes the proof.

In the next result, we prove the existence of solutions for the problem (1.1) by applying Leray-Schauder alternative.

Lemma 3.1 (Leray-Schauder alternative [7]). Let $F: E \rightarrow E$ be a completely continuous operator (i.e., a map that restricted to any bounded set in $E$ is compact). Let

$$
\aleph(F)=\{x \in E \mid x=\lambda F(x) \text { for some } 0<\lambda<1\} .
$$

Then either the set $\aleph(F)$ is unbounded or $F$ has at least one fixed point.

Theorem 3.2. Assume that there exist real constants $k_{i}, \lambda_{i}>0, i=1,2$, and $k_{0}>$ $0, \lambda_{0}>0$ such that for all $x_{i} \in \mathbb{R}, i=1,2$, we have

$$
\left|f\left(t, x_{1}, x_{2}\right)\right| \leq k_{0}+k_{1}\left|x_{1}\right|+k_{2}\left|x_{2}\right|
$$

and

$$
\left|g\left(t, x_{1}, x_{2}\right)\right| \leq \lambda_{0}+\lambda_{1}\left|x_{1}\right|+\lambda_{2}\left|x_{2}\right|
$$

In addition, also assume that

$$
\left(M_{1} k_{1}+M_{2} \lambda_{1}\right) \leq 1 \quad \text { and } \quad\left(M_{1} k_{2}+M_{2} \lambda_{2}\right) \leq 1,
$$

where $M_{i}, i=1,2$, are given by (3.3) and (3.4). Then there exists at least one solution for the boundary value problem (1.1).

Proof. First we show that the operator $\mathcal{T}: X \times Y \rightarrow X \times Y$ is completely continuous. By continuity of functions $\mathrm{f}$ and $\mathrm{g}$ the operator $\mathcal{T}$ is continuous.

Let $\Theta \subset X \times Y$ be bounded. Then there exist positive constants $L_{1}$ and $L_{2}$ such that

$$
|f(t, u(t), v(t))| \leq L_{1}, \quad|g(t, u(t), v(t))| \leq L_{2}, \quad \text { for all }(u, v) \in \Theta .
$$

For any $(u, v) \in \Theta$, we have

$$
\begin{aligned}
\left\|\mathcal{T}_{1}(u, v)(t)\right\| \leq & \frac{1}{\left|A_{1}\right|}\left(\int_{1}^{t} s^{k-1}(\log s)^{q-2} d s\right)\left[\sum_{i=1}^{m} \frac{\left|\lambda_{i}\right|}{\Gamma\left(\alpha_{i}\right) \Gamma(q-1)} \int_{1}^{\eta_{i}}\left(\log \frac{\eta_{i}}{s}\right)^{\alpha_{i}-1} s^{-k-1}\right. \\
& \times\left(\int_{1}^{s} r^{k-1}\left(\int_{1}^{r}\left(\log \frac{r}{m}\right)^{q-2} \frac{|f(m, u(m), v(m))|}{m} d m\right) d r\right) d s \\
& +\sum_{j=1}^{n} \frac{\left|\mu_{j}\right|}{\Gamma\left(\beta_{j}\right) \Gamma(q-1)} \int_{1}^{\xi_{j}}\left(\log \frac{\xi_{j}}{s}\right)^{\beta_{j}-1} s^{-k-1} \\
& \times\left(\int_{1}^{s} r^{k-1}\left(\int_{1}^{r}\left(\log \frac{r}{m}\right)^{q-2} \frac{|f(m, u(m), v(m))|}{m} d m\right) d r\right) d s
\end{aligned}
$$




$$
\begin{aligned}
& +\sum_{j=1}^{n} \frac{\left|\mu_{j}\right|}{\Gamma\left(\beta_{j}\right) \Gamma(q-1)} \int_{1}^{e}\left(\log \frac{e}{s}\right)^{\beta_{j}-1} s^{-1} \\
& \left.\times\left(\int_{1}^{e} r^{k-1}\left(\int_{1}^{r}\left(\log \frac{r}{m}\right)^{q-2} \frac{(|f(m, u(m), v(m))|}{m} d m\right) d r\right) d s\right] \\
& +\frac{1}{\Gamma(q-1)} \int_{1}^{t} s^{k-1}\left(\int_{1}^{s}\left(\log \frac{s}{r}\right)^{q-2} \frac{(|f(r, u(r), v(r))|}{r} d r\right) d s \\
\leq & \frac{L_{1}}{\left|A_{1}\right|}\left(\int_{1}^{t} s^{k-1}(\log s)^{q-2} d s\right)\left[\sum_{i=1}^{m} \frac{\left|\lambda_{i}\right|}{\Gamma\left(\alpha_{i}\right) \Gamma(q-1)} \int_{1}^{\eta_{i}}\left(\log \frac{\eta_{i}}{s}\right)^{\alpha_{i}-1} s^{-k-1}\right. \\
& \times\left(\int_{1}^{s} r^{k-1}\left(\int_{1}^{r}\left(\log \frac{r}{m}\right)^{q-2} \frac{1}{m} d m\right) d r\right) d s \\
& +\sum_{j=1}^{n} \frac{\left|\mu_{j}\right|}{\Gamma\left(\beta_{j}\right) \Gamma(q-1)} \int_{1}^{\xi_{j}}\left(\log \frac{\xi_{j}}{s}\right)^{\beta_{j}-1} s^{-k-1} \\
& \times\left(\int_{1}^{s} r^{k-1}\left(\int_{1}^{r}\left(\log \frac{r}{m}\right)^{q-2} \frac{1}{m} d m\right) d r\right) d s \\
& +\sum_{j=1}^{n} \frac{\left|\mu_{j}\right|}{\Gamma\left(\beta_{j}\right) \Gamma(q-1)} \int_{1}^{e}\left(\log \frac{e}{s}\right)^{\beta_{j}-1} s^{-1} \\
& \left.\times\left(\int_{1}^{e} r^{k-1}\left(\int_{1}^{r}\left(\log \frac{r}{m}\right)^{q-2} \frac{1}{m} d m\right) d r\right) d s\right] \\
& +\frac{L_{1}}{\Gamma(q-1)} \int_{1}^{t} s^{k-1}\left(\int_{1}^{s}\left(\log \frac{s}{r}\right)^{q-2} \frac{1}{r} d r\right) d s
\end{aligned}
$$

which implies that

$$
\begin{aligned}
\left\|\mathcal{T}_{1}(u, v)(t)\right\| \leq & L_{1}\left\{\frac { 1 } { | A _ { 1 } | } \left(\sum_{i=1}^{m} \frac{\left|\lambda_{i}\right|\left(\log \eta_{i}\right)^{\alpha_{i}+q}}{(q-1) \Gamma\left(\alpha_{i}+1\right) \Gamma(q+1)}\right.\right. \\
& \left.+\sum_{j=1}^{n} \frac{\left|\mu_{j}\right|\left(\log \xi_{j}\right)^{\beta_{j}+q}}{(q-1) \Gamma\left(\beta_{j}+1\right) \Gamma(q+1)}\right) \\
& \left.+\sum_{j=1}^{n} \frac{\left|\mu_{j}\right|}{(q-1) \Gamma\left(\beta_{j}+1\right) \Gamma(q+1)}\right\} \\
= & L_{1} M_{1} .
\end{aligned}
$$

Similarly, we get

$$
\begin{aligned}
\left\|\mathcal{T}_{2}(u, v)(t)\right\| \leq & L_{2}\left\{\frac { 1 } { | A _ { 2 } | } \left(\sum_{i=1}^{m} \frac{\left|\rho_{i}\right|\left(\log \theta_{i}\right)^{\gamma_{i}+p}}{(p-1) \Gamma\left(\gamma_{i}+1\right) \Gamma(p+1)}\right.\right. \\
& \left.+\sum_{j=1}^{n} \frac{\left|\kappa_{j}\right|\left(\log \zeta_{j}\right)^{\delta_{j}+p}}{(p-1) \Gamma\left(\delta_{j}+1\right) \Gamma(p+1)}\right)
\end{aligned}
$$




$$
\begin{aligned}
& \left.+\sum_{j=1}^{n} \frac{\left|\kappa_{j}\right|}{(p-1) \Gamma\left(\delta_{j}+1\right) \Gamma(p+1)}\right\} \\
= & L_{2} M_{2} .
\end{aligned}
$$

Thus, it follows from the above inequalities that the operator $\mathcal{T}$ is uniformly bounded.

Next, we show that $\mathcal{T}$ is equicontinuous. Let $t_{1}, t_{2} \in[1, e]$ with $t_{1}<t_{2}$. Then we have

$$
\begin{aligned}
\mid & \mathcal{T}_{1}\left(u\left(t_{2}\right), v\left(t_{2}\right)\right)-\mathcal{T}_{1}\left(u\left(t_{1}\right), v\left(t_{1}\right)\right) \mid \\
\leq & \frac{L_{1}}{\left|A_{1}\right|}\left(\frac{\left|t_{1}^{k}-t_{2}^{k}\right|}{t_{1}^{k} t_{2}^{k}} \int_{1}^{t_{1}} s^{k-1}(\log s)^{q-2} d s+t_{2}^{-k} \int_{t_{1}}^{t_{2}} s^{k-1}(\log s)^{q-2} d s\right) \\
& \times\left[\sum_{i=1}^{m} \frac{\left|\lambda_{i}\right|}{\Gamma\left(\alpha_{i}\right) \Gamma(q-1)} \int_{1}^{\eta_{i}}\left(\log \frac{\eta_{i}}{s}\right)^{\alpha_{i}-1} s^{-k-1}\right. \\
& \times\left(\int_{1}^{s} r^{k-1}\left(\int_{1}^{r}\left(\log \frac{r}{m}\right)^{q-2} \frac{1}{m} d m\right) d r\right) d s \\
& +\sum_{j=1}^{n} \frac{\left|\mu_{j}\right|}{\Gamma\left(\beta_{j}\right) \Gamma(q-1)} \int_{1}^{\xi_{j}}\left(\log \frac{\xi_{j}}{s}\right)^{\beta_{j}-1} s^{-k-1} \\
& \times\left(\int_{1}^{s} r^{k-1}\left(\int_{1}^{r}\left(\log \frac{r}{m}\right)^{q-2} \frac{1}{m} d m\right) d r\right) d s \\
& +\sum_{j=1}^{n} \frac{\left|\mu_{j}\right|}{\Gamma\left(\beta_{j}\right) \Gamma(q-1)} \int_{1}^{e}\left(\log \frac{e}{s}\right)^{\beta_{j}-1} s^{-1} \\
& \left.\times\left(\int_{1}^{e} r^{k-1}\left(\int_{1}^{r}\left(\log \frac{r}{m}\right)^{q-2} \frac{1}{m} d m\right) d r\right) d s\right] \\
& +\frac{L_{1}}{\Gamma(q-1)}\left[\frac{\left|t_{1}^{k}-t_{2}^{k}\right|}{t_{1}^{k} t_{2}^{k}} \int_{1}^{t_{1}} s^{k-1}\left(\int_{1}^{s}\left(\log \frac{s}{r}\right)^{q-2} \frac{1}{r} d r\right) d s\right. \\
& \left.+t_{2}^{-k} \int_{t_{1}}^{t_{2}} s^{k-1}\left(\int_{1}^{s}\left(\log \frac{s}{r}\right)^{q-2} \frac{1}{r} d r\right) d s\right],
\end{aligned}
$$

which implies that

$$
\left|\mathcal{T}_{1}\left(u\left(t_{2}\right), v\left(t_{2}\right)\right)-\mathcal{T}_{1}\left(u\left(t_{1}\right), v\left(t_{1}\right)\right)\right| \rightarrow 0 \quad \text { as } \quad t_{2}-t_{1} \rightarrow 0
$$

Analogously, we can obtain

$$
\left|\mathcal{T}_{2}\left(u\left(t_{2}\right), v\left(t_{2}\right)\right)-\mathcal{T}_{2}\left(u\left(t_{1}\right), v\left(t_{1}\right)\right)\right| \rightarrow 0 \quad \text { as } \quad t_{2}-t_{1} \rightarrow 0
$$

From (3.9) and (3.10), it is obvious that $\mathcal{T}$ is equicontinuous and thus is completely continuous.

Finally, it will be verified that the set $\aleph=\{(u, v) \in X \times Y:(u, v)=\lambda \mathcal{T}(u, v), 0 \leq$ $\lambda \leq 1\}$ is bounded. Let $(u, v) \in \aleph$, then $(u, v)=\lambda \mathcal{T}(u, v)$. For any $t \in[1, e]$, we have

$$
u(t)=\lambda \mathcal{T}_{1}(u, v)(t), \quad v(t)=\lambda \mathcal{T}_{2}(u, v)(t) .
$$


Then

$$
\begin{aligned}
|u(t)| \leq & \left(k_{0}+k_{1}\|u\|+k_{2}\|v\|\right)\left[\frac { 1 } { | A _ { 1 } | } \left(\sum_{i=1}^{m} \frac{\left|\lambda_{i}\right|\left(\log \eta_{i}\right)^{\alpha_{i}+q}}{(q-1) \Gamma\left(\alpha_{i}+1\right) \Gamma(q+1)}\right.\right. \\
& \left.\left.+\sum_{j=1}^{n} \frac{\left|\mu_{j}\right|\left(\log \xi_{j}\right)^{\beta_{j}+q}}{(q-1) \Gamma\left(\beta_{j}+1\right) \Gamma(q+1)}\right)+\sum_{j=1}^{n} \frac{\left|\mu_{j}\right|}{(q-1) \Gamma\left(\beta_{j}+1\right) \Gamma(q+1)}\right]
\end{aligned}
$$

and

$$
\begin{aligned}
|v(t)| \leq & \left(\lambda_{0}+\lambda_{1}\|u\|+\lambda_{2}\|v\|\right)\left[\frac { 1 } { | A _ { 2 } | } \left(\sum_{i=1}^{m} \frac{\left|\rho_{i}\right|\left(\log \theta_{i}\right)^{\gamma_{i}+p}}{(p-1) \Gamma\left(\gamma_{i}+1\right) \Gamma(p+1)}\right.\right. \\
& \left.\left.+\sum_{j=1}^{n} \frac{\left|\kappa_{j}\right|\left(\log \zeta_{j}\right)^{\delta_{j}+p}}{(p-1) \Gamma\left(\delta_{j}+1\right) \Gamma(p+1)}\right)+\sum_{j=1}^{n} \frac{\left|\kappa_{j}\right|}{(p-1) \Gamma\left(\delta_{j}+1\right) \Gamma(p+1)}\right] .
\end{aligned}
$$

Hence, we have

$$
\|u(t)\| \leq\left(k_{0}+k_{1}\|u\|+k_{2}\|v\|\right) M_{1}
$$

and

$$
\|v(t)\| \leq\left(\lambda_{0}+\lambda_{1}\|u\|+\lambda_{2}\|v\|\right) M_{2}
$$

which implies that

$$
\|u(t)\|+\|v(t)\| \leq\left(M_{1} k_{0}+M_{2} \lambda_{0}\right)+\left(M_{1} k_{1}+M_{2} \lambda_{1}\right)\|u\|+\left(M_{1} k_{2}+M_{2} \lambda_{2}\right)\|v\| .
$$

Consequently,

$$
\|(u, v)\| \leq \frac{M_{1} k_{0}+M_{2} \lambda_{0}}{M_{0}},
$$

for any $t \in[1, e]$, where $M_{0}$ is defined by (3.5), which proves that $\aleph$ is bounded. Thus, by Lemma 3.1 the operator $\mathcal{T}$ has at least one fixed point. Hence, the boundary value problem (1.1) has at least one solution. The proof is complete.

\section{Hyers-Ulam Stability of System (1.1)}

This section is devoted to the investigation of the Hyers-Ulam stability of our proposed system. Let $\varepsilon_{1}, \varepsilon_{2}>0$ such that:

$$
\left\{\begin{array}{l}
\left|\left(D^{q}+k D^{q-1}\right) u(t)-f(t, u(t), v(t))\right| \leq \varepsilon_{1}, \quad t \in[1, e] \\
\left|\left(D^{p}+k D^{p-1}\right) v(t)-g(t, u(t), v(t))\right| \leq \varepsilon_{2}, \quad t \in[1, e]
\end{array}\right.
$$

Definition 4.1. Problem (1.1) is said to be Hyers-Ulam stable if there exist $M_{i}>0$, $i=1,2$, such that, for given $\varepsilon_{1}, \varepsilon_{2}>0$ and for each solution $(u, v) \in C\left([1, e] \times \mathbb{R}^{\sharp}, \mathbb{R}\right)$ of inequality (4.1), there exists a solution $\left(u^{*}, v^{*}\right) \in C\left([1, e] \times \mathbb{R}^{\not}, \mathbb{R}\right)$ of problem (1.1) with

$$
\left\{\begin{array}{l}
\left|u(t)-u^{*}(t)\right| \leq M_{1} \varepsilon_{1}, \quad t \in[1, e], \\
\left|u(t)-v^{*}(t)\right| \leq M_{2} \varepsilon_{2}, \quad t \in[1, e] .
\end{array}\right.
$$

Remark 4.1. A $(u, v)$ is a solution of inequality (4.1) if there exist functions $Q_{i} \in$ $C([1, e], \mathbb{R}), i=1,2$ which depend on $u, v$ respectively such that 
- $\left|Q_{1}(t)\right| \leq \varepsilon_{1},\left|Q_{2}(t)\right| \leq \varepsilon_{2}, t \in[1, e]$, and

$$
\begin{cases}\left(D^{q}+k D^{q-1}\right) u(t)=f(t, u(t), v(t))+Q_{1}(t), & t \in[1, e], \\ \left(D^{p}+k D^{p-1}\right) v(t)=g(t, u(t), v(t))+Q_{2}(t), & t \in[1, e] .\end{cases}
$$

Remark 4.2. If $(x, y)$ represents a solution of inequality $(4.1)$, then $(x, y)$ is a solution of following inequality:

$$
\left\{\begin{array}{l}
\left|x(t)-x^{*}(t)\right| \leq M_{1} \varepsilon_{1}, \quad t \in[1, e] \\
\left|y(t)-y^{*}(t)\right| \leq M_{2} \varepsilon_{2}, \quad t \in[1, e] .
\end{array}\right.
$$

As from Remark 4.1, we have

$$
\begin{cases}\left(D^{q}+k D^{q-1}\right) u(t)=f(t, u(t), v(t))+Q_{1}(t), & t \in[1, e] \\ \left(D^{p}+k D^{p-1}\right) v(t)=g(t, u(t), v(t))+Q_{2}(t), & t \in[1, e]\end{cases}
$$

With the help of Definition 4.1 and Remark 4.1, we verified Remark 4.2, in the following lines

$$
\begin{aligned}
\mid u(t)-\frac{1}{A_{1}}\left(t^{-k} \int_{1}^{t} s^{k-1}(\log s)^{q-2} d s\right)\left[\sum_{i=1}^{m} \frac{\lambda_{i}}{\Gamma\left(\alpha_{i}\right) \Gamma(q-1)} \int_{1}^{\eta_{i}}\left(\log \frac{\eta_{i}}{s}\right)^{\alpha_{i}-1} s^{-k-1}\right. \\
\times\left(\int_{1}^{s} r^{k-1}\left(\int_{1}^{r}\left(\log \frac{r}{m}\right)^{q-2} \frac{f(m, u(m), v(m))}{m} d m\right) d r\right) d s \\
+\sum_{j=1}^{n} \frac{\mu_{j}}{\Gamma\left(\beta_{j}\right) \Gamma(q-1)} \int_{1}^{\xi_{j}}\left(\log \frac{\xi_{j}}{s}\right)^{\beta_{j}-1} s^{-k-1} \\
\times\left(\int_{1}^{s} r^{k-1}\left(\int_{1}^{r}\left(\log \frac{r}{m}\right)^{q-2} \frac{f(m, u(m), v(m))}{m} d m\right) d r\right) d s \\
-\sum_{j=1}^{n} \frac{e^{-k} \mu_{j}}{\Gamma\left(\beta_{j}\right) \Gamma(q-1)} \int_{1}^{e}\left(\log \frac{e}{s}\right)^{\beta_{j}-1} s^{-1} \\
\left.\quad \times\left(\int_{1}^{e} r^{k-1}\left(\int_{1}^{r}\left(\log \frac{r}{m}\right)^{q-2} \frac{f(m, u(m), v(m))}{m} d m\right) d r\right) d s\right] \\
-\frac{t^{-k}}{\Gamma(q-1)} \int_{1}^{t} s^{k-1}\left(\int_{1}^{s}\left(\log \frac{s}{r}\right)^{q-2} \frac{f(r, u(r), v(r))}{r} d r\right) d s \mid \\
=\mid \frac{1}{A_{1}}\left(t^{-k} \int_{1}^{t} s^{k-1}(\log s)^{q-2} d s\right)\left[\sum_{i=1}^{m} \frac{\lambda_{i}}{\Gamma\left(\alpha_{i}\right) \Gamma(q-1)} \int_{1}^{\eta_{i}}\left(\log \frac{\eta_{i}}{s}\right)^{\alpha_{i}-1} s^{-k-1}\right. \\
\quad \times\left(\int_{1}^{s} r^{k-1}\left(\int_{1}^{r}\left(\log \frac{r}{m}\right)^{q-2} \frac{\left[f(m, u(m), v(m))+Q_{1}(m)\right]}{m} d m\right) d r\right) d s \\
\quad+\sum_{j=1}^{n} \frac{\mu_{j}}{\Gamma\left(\beta_{j}\right) \Gamma(q-1)} \int_{1}^{\xi_{j}}\left(\log \frac{\xi_{j}}{s}\right)^{\beta_{j}-1} s^{-k-1}
\end{aligned}
$$




$$
\begin{aligned}
& \times\left(\int_{1}^{s} r^{k-1}\left(\int_{1}^{r}\left(\log \frac{r}{m}\right)^{q-2} \frac{\left[f(m, u(m), v(m))+Q_{1}(m)\right]}{m} d m\right) d r\right) d s \\
& -\sum_{j=1}^{n} \frac{e^{-k} \mu_{j}}{\Gamma\left(\beta_{j}\right) \Gamma(q-1)} \int_{1}^{e}\left(\log \frac{e}{s}\right)^{\beta_{j}-1} s^{-1} \\
& \left.\times\left(\int_{1}^{e} r^{k-1}\left(\int_{1}^{r}\left(\log \frac{r}{m}\right)^{q-2} \frac{\left[f(m, u(m), v(m))+Q_{1}(m)\right]}{m} d m\right) d r\right) d s\right] \\
& +\frac{t^{-k}}{\Gamma(q-1)} \int_{1}^{t} s^{k-1}\left(\int_{1}^{s}\left(\log \frac{s}{r}\right)^{q-2} \frac{\left[f(r, u(r), v(r))+Q_{1}(r)\right]}{r} d r\right) d s \\
& -\frac{1}{A_{1}}\left(t^{-k} \int_{1}^{t} s^{k-1}(\log s)^{q-2} d s\right)\left[\sum_{i=1}^{m} \frac{\lambda_{i}}{\Gamma\left(\alpha_{i}\right) \Gamma(q-1)} \int_{1}^{\eta_{i}}\left(\log \frac{\eta_{i}}{s}\right)^{\alpha_{i}-1} s^{-k-1}\right. \\
& \times\left(\int_{1}^{s} r^{k-1}\left(\int_{1}^{r}\left(\log \frac{r}{m}\right)^{q-2} \frac{f(m, u(m), v(m))}{m} d m\right) d r\right) d s \\
& +\sum_{j=1}^{n} \frac{\mu_{j}}{\Gamma\left(\beta_{j}\right) \Gamma(q-1)} \int_{1}^{\xi_{j}}\left(\log \frac{\xi_{j}}{s}\right)^{\beta_{j}-1} s^{-k-1} \\
& \times\left(\int_{1}^{s} r^{k-1}\left(\int_{1}^{r}\left(\log \frac{r}{m}\right)^{q-2} \frac{f(m, u(m), v(m))}{m} d m\right) d r\right) d s \\
& -\sum_{j=1}^{n} \frac{e^{-k} \mu_{j}}{\Gamma\left(\beta_{j}\right) \Gamma(q-1)} \int_{1}^{e}\left(\log \frac{e}{s}\right)^{\beta_{j}-1} s^{-1} \\
& \left.\times\left(\int_{1}^{e} r^{k-1}\left(\int_{1}^{r}\left(\log \frac{r}{m}\right)^{q-2} \frac{f(m, u(m), v(m))}{m} d m\right) d r\right) d s\right] \\
& -\frac{t^{-k}}{\Gamma(q-1)} \int_{1}^{t} s^{k-1}\left(\int_{1}^{s}\left(\log \frac{s}{r}\right)^{q-2} \frac{f(r, u(r), v(r))}{r} d r\right) d s \mid \\
& \leq \varepsilon_{1}\left[\frac{1}{\left|A_{1}\right|}\left(\sum_{i=1}^{m} \frac{\left|\lambda_{i}\right|\left(\log \eta_{i}\right)^{\alpha_{i}+q}}{(q-1) \Gamma\left(\alpha_{i}+1\right) \Gamma(q+1)}+\sum_{j=1}^{n} \frac{\left|\mu_{j}\right|\left(\log \xi_{j}\right)^{\beta_{j}+q}}{(q-1) \Gamma\left(\beta_{j}+1\right) \Gamma(q+1)}\right)\right. \\
& \left.+\sum_{j=1}^{n} \frac{\left|\mu_{j}\right|}{(q-1) \Gamma\left(\beta_{j}+1\right) \Gamma(q+1)}\right] \\
& +\varepsilon_{2}\left[\frac{1}{\left|A_{2}\right|}\left(\sum_{i=1}^{m} \frac{\left|\rho_{i}\right|\left(\log \theta_{i}\right)^{\gamma_{i}+p}}{(p-1) \Gamma\left(\gamma_{i}+1\right) \Gamma(p+1)}+\sum_{j=1}^{n} \frac{\left|\kappa_{j}\right|\left(\log \zeta_{j}\right)^{\delta_{j}+p}}{(p-1) \Gamma\left(\delta_{j}+1\right) \Gamma(p+1)}\right)\right. \\
& \left.+\sum_{j=1}^{n} \frac{\left|\kappa_{j}\right|}{(p-1) \Gamma\left(\delta_{j}+1\right) \Gamma(p+1)}\right] \\
& =M_{1} \varepsilon_{1} \text {. }
\end{aligned}
$$


By the same method, we can obtain that

$$
\left|y(t)-y^{*}(t)\right| \leq M_{2} \varepsilon_{2},
$$

where $M_{i}, i=1,2$, are given by (3.3) and (3.4). Hence, Remark 4.2 is verified, with the help of (4.4) and (4.5). Thus the nonlinear sequential coupled system of Hadamard fractional differential equations is Hyers-Ulam stable and consequently, the system (1.1) is Hyers-Ulam stable.

Example 4.1. Consider the following coupled system of Hadamard fractional differential equation:

$$
\left\{\begin{array}{l}
\left(D^{3 / 2}+3 D^{1 / 2}\right) u(t)=\frac{|u(t)|}{(t+3)^{4}+(1+|u(t)|)}+\frac{1}{27(1+|v(t)|)}+\frac{1}{81}, \quad t \in[1, e], \\
\left(D^{3 / 2}+3 D^{1 / 2}\right) v(t)=\frac{\sin (2 \pi|u(t)|)}{40 \pi}+\frac{1}{10 \sqrt{t+4}}+\frac{|v(t)|}{60(1+|v(t)|)}, \quad t \in[1, e], \\
u(1)=0, \\
2 I^{1 / 4} u(5 / 4)+\frac{1}{5} I^{3 / 2} u(9 / 5)+3 I^{1 / 2} u(15 / 7) \\
=I^{2 / 3} u(e)-I^{2 / 3} u(10 / 7)+5\left(I^{9 / 7} u(e)-I^{9 / 7} u(2)\right)-2\left(I^{11 / 4} u(e)-I^{11 / 4} u(9 / 4)\right), \\
v(1)=0, \\
\frac{1}{4} I^{7 / 6} v(7 / 3)-\frac{2}{3} I^{1 / 2} v(7 / 5)-2 I^{5 / 2} v(2) \\
=4\left(I^{5} v(e)-I^{5} v(11 / 5)\right)+\frac{11}{4}\left(I^{3 / 4} v(e)-I^{3 / 4} v(13 / 16)\right)-\frac{1}{2}\left(I^{7 / 4} v(e)\right. \\
\left.-I^{7 / 4} v(1 / 3)\right) .
\end{array}\right.
$$

Here, $q=p=3 / 2, n=3, m=3, k=3, \lambda_{1}=2, \lambda_{2}=1 / 5, \lambda_{3}=3, \alpha_{1}=1 / 4$, $\alpha_{2}=3 / 2, \alpha_{3}=1 / 2, \eta_{1}=5 / 4, \eta_{2}=9 / 5, \eta_{3}=15 / 7, \mu_{1}=1, \mu_{2}=5, \mu_{3}=-2$, $\beta_{1}=2 / 3, \beta_{2}=9 / 7, \beta_{3}=11 / 4, \xi_{1}=10 / 7, \xi_{2}=2, \xi_{3}=9 / 4, \rho_{1}=1 / 4, \rho_{2}=-2 / 3$, $\rho_{3}=-2, \gamma_{1}=7 / 6, \gamma_{2}=1 / 2, \gamma_{3}=5 / 2, \theta_{1}=7 / 3, \theta_{2}=7 / 5, \theta_{3}=2, \kappa_{1}=4, \kappa_{2}=11 / 4$, $\kappa_{3}=-1 / 2, \delta_{1}=5, \delta_{2}=3 / 4, \delta_{3}=7 / 4, \zeta_{1}=11 / 5, \zeta_{2}=13 / 16, \zeta_{3}=1 / 3$. Thus,

$$
f(t, u, v)=(u(t)) /\left((t+3)^{4}+(1+|u(t)|)\right)+1 /(27(1+|v(t)|))+1 / 81
$$

and

$$
g(t, u, v)=(\sin (2 \pi|u(t)|)) / 40 \pi+1 /(10 \sqrt{t+4})+(|v(t)|) /(60(1+|v(t)|)),
$$

which implies

$$
\left|f\left(t, u_{2}, v_{2}\right)-f\left(t, u_{1}, v_{1}\right)\right| \leq(1 / 81)\left|u_{2}-u_{1}\right|+(1 / 27)\left|v_{2}-v_{1}\right|
$$

and

$$
\left|g\left(t, u_{2}, v_{2}\right)-g\left(t, u_{1}, v_{1}\right)\right| \leq(1 / 20)\left|u_{2}-u_{1}\right|+(1 / 60)\left|v_{2}-v_{1}\right| .
$$

Clearly, $m_{1}=1 / 81, m_{2}=1 / 27, n_{1}=1 / 20, n_{2}=1 / 60, M_{1} \simeq 5.695, M_{2} \simeq 6.785$, and

$$
\left[M_{1}\left(m_{1}+m_{2}\right)+M_{2}\left(n_{1}+n_{2}\right)\right] \simeq 0.732<1 .
$$


Thus, all the conditions of Theorem 3.1 are satisfied. Therefore, by Theorem 3.1, the problem (4.8) has a unique solution on $[1, e]$. Further, it is also straightforward to prove that the problem (4.8) is Hyers-Ullam stable.

\section{Conclusion}

We have discussed the existence and Hyers-Ulam stability for a sequential coupled system consisting of fractional differential equations of Hadamard type, subjected to nonlocal Hadamard fractional integral boundary conditions. The existence and uniqueness of solutions rely on Banach's contraction principle, while the existence of solutions is established by applying Leray-Schauder's alternative. As an application, an example is presented to illustrate the main results.

\section{REFERENCES}

[1] G. Adomian and G. E. Adomian, Cellular systems and aging models, Comput. Math. Appl. 11 (1985), 283-291.

[2] B. Ahmad, J. Juan, J. Nieto and A. Alseadi, A coupled system of Caputo-type sequential fractional differential equations with coupled (periodic/anti-periodic type) boundary conditions, Mediterr. J. Math. 2017 (2017), 227.

[3] B. Ahmad and S. K. Ntouayas, A fully Hadamard type integral boundary value problome of a coupled system of fractional differential equations, Fract. Calc. Appl. Anal. 17 (2014), 348-360.

[4] Z. Ali, A. Zada and K. Shah, On Ulam's stability for a coupled systems of nonlinear implicit fractional differential equations, Bull. Malays. Math. Sci. Soc. 42 (2019), 2681-2699.

[5] R. L. Bagley and P. J. Torvik, A theoretical basis for the application of fractional calculus to viscoelasticity, Journal of Rheology 27 (1983), 201-210.

[6] K. W. Blayneh, Analysis of age structured host-parasitoid model, Far East Journal of Dynamical Systems 4 (2002), 125-145.

[7] A. Granas and J. Dugundji, Fixed Point Theory, Springer-Verlag, New York, 2013.

[8] J. Hadamard, Essai sur l'etude des fonctions donnes par leur developpment de taylor, Journal des Mathématiques Pures et Appliquées 8 (1892), 86-101.

[9] R. Hilfer, Applications of Fractional Calculus in Physics, World Scientific, SIngapore, 2000.

[10] D. H. Hyers, On the stability of the linear functional equation, Proc. Natl. Acad. Sci. USA 27 (1941), 222-224.

[11] S. Karthikeyan, C. Ravichandran and T. Gunasekar, Existence results for Hadamard type fractional functional integro-differential equations with integral boundary conditions, International Journal of Engineering Research 10 (2015), 6919-6932.

[12] H. Khan, Y. Li, W. Chen, D. Baleanu and A. Khan, Existence theorems and Hyers-Ulam stability for a coupled system of fractional differential equations with p-Laplacian operator, Bound. Value Probl. 2017.

[13] H. Khan, Y. Li, H. Hongguang and A. Khan, Existence of solution and Hyers-Ulam stability for a coupled system of fractional differential equations with p-laplacian operator, J. Nonlinear Sci. Appl. 10 (2017), 5219-5229.

[14] A. A. Kilbas, H. M. Srivastava and J. J. Trujillo, Theory and Applications of Fractional Differential Equations, North-Holland Mathematics Studies, Amsterdam, 2016.

[15] D. Matignon, Stability results for fractional differential equations with applications to control processing, Computational Engineering in System Application 2 (1996), 963-968. 
[16] T. Phollakrit, S. K. Ntouyas and T. Jessada, Existence and uniqueness results for Hadamardtype fractional differential equations with nonlocal fractional integral boundary conditions, Abstr. Appl. Anal. 5 (2014), 1-9.

[17] I. Podlubny, Fractional Differential Equations, Academic Press, San Diego, 1999.

[18] J. Sabatier, O. P. Agrawal and J. A. T. Machado, Advances in Fractional Calculus, Theoretical Developments and Applications in Physics and Engineering, Springer, Dordrecht, 2007.

[19] S. G. Samko, A. A. Kilbas and O. l. Marichev, Fractional Integrals and Derivatives: Theory and Applications, Gordon and Breach, Yverdon, 1993.

[20] K. Shah and R. A. Khan, Existence and uniqueness of positive solutions to a coupled system of nonlinear fractional order differential equations with anti periodic boundary conditions, Differ. Equ. Appl. 7(2) (2015), 245-262.

[21] R. Shah and A. Zada, A fixed point approach to the stability of a nonlinear Volterra integrodiferential equation with delay, Hacet. J. Math. Stat. 47 (2018), 615-623.

[22] S. O. Shah and A. Zada, Existence, uniqueness and stability of solution to mixed integral dynamic systems with instantaneous and noninstantaneous impulses on time scales, Appl. Math. Comput. 359 (2019), 202-213.

[23] S. O. Shah, A. Zada and A. E. Hamza, Stability analysis of the first order non-linear impulsive time varying delay dynamic system on time scales, Qual. Theory Dyn. Syst. (2019), DOI $10.1007 / \mathrm{s} 12346-019-00315-\mathrm{x}$.

[24] S. Tang, A. Zada, S. Faisal, M. M. A. El-Sheikh and T. Li, Stability of higher-order nonlinear impulsive differential equations, J. Nonlinear Sci. Appl. 9 (2016), 4713-4721.

[25] J. Tariboon and W. Sudsutad, Coupled systems of Riemann-Liouville fractional differential equations with Hadamard fractional integral boundary conditions, J. Nonlinear Sci. Appl. 9 (2016), 295-308.

[26] S. M. Ulam, Problems in Modern Mathematics, John Wiley and Sons, New York, USA, 1940.

[27] J. Wang, L. Lv and Y. Zhou, Ulam stability and data dependence for fractional differential equations with Caputo derivative, Electron J. Qual. Theo. Diff. Equns. 63 (2011), 1-10.

[28] J. Wang, A. Zada and W. Ali, Ulam's-type stability of first-order impulsive differential equations with variable delay in quasi-Banach spaces, Numer. Funct. Anal. Optim. 33 (2012), 216-238.

[29] J. Wang, A. Zada and W. Ali, Ulam's-type stability of first-order impulsive differential equations with variable delay in quasi-Banach spaces, Int. J. Nonlinear Sci. 19 (2018), 553-560.

[30] A. Zada and S. Ali, Stability analysis of multi-point boundary value problem for sequential fractional differential equations with non-instantaneous impulses, Int. J. Nonlinear Sci. Numer. Simul. 19 (2018), 763-774.

[31] A. Zada, S. Ali and Y. Li, Ulam-type stability for a class of implicit fractional differential equations with non-instantaneous integral impulses and boundary condition, Adv. Difference Equ. 2017.

[32] A. Zada, W. Ali and S. Farina, Hyers-Ulam stability of nonlinear differential equations with fractional integrable impulses, Math. Methods Appl. Sci. 40 (2017), 5502-5514.

[33] A. Zada, W. Ali and C. Park, Ulam's type stability of higher order nonlinear delay differential equations via integral inequality of Grönwall-Bellman-Bihari's type, Appl. Math. Comput. 350 (2019), 60-65.

[34] A. Zada, U. Riaz and F. U. Khan, Hyers-Ulam stability of impulsive integral equations, Boll. Unione Mat. Ital. 12 (2019), 453-467.

[35] A. Zada and S. O. Shah, Hyers-Ulam stability of first-order non-linear delay differential equations with fractional integrable impulses, Hacet. J. Math. Stat. 47 (2018), 1196-1205.

[36] A. Zada, S. O. Shah and R. Shah, Hyers-Ulam stability of non-autonomous systems in terms of boundedness of Cauchy problem, Appl. Math. Comput. 271 (2015), 512-518.

[37] A. Zada, S. Shaleena and T. Li, Stability analysis of higher order nonlinear differential equations in $\beta$-normed spaces, Math. Methods Appl. Sci. 42 (2019), 1151-1166. 
[38] A. Zada, P. Wang, D. Lassoued and T. Li, Connections between Hyers-Ulam stability and uniform exponential stability of 2-periodic linear nonautonomous systems, Adv. Difference Equ. 2017.

[39] A. Zada, M. Yar and T. Li, Existence and stability analysis of nonlinearsequential coupled system of Caputo fractional differential equations with integral boundary conditions, Ann. Univ. Paedagog. Crac. Stud. Math. 17 (2018), 103-125.

${ }^{1}$ Department of Mathematics, University of PeshaWAR, Peshawar 25000, PAKISTAN

Email address: zadababo@yahoo.com, akbarzada@uop.edu.pk

Email address: mohammadyar2030@gmail.com 
Kragujevac Journal of Mathematics

Volume 46(1) (2022), Pages 105-113.

\title{
EXTREMAL GRAPHS FOR EXPONENTIAL VDB INDICES
}

\author{
ROBERTO CRUZ ${ }^{1}$ AND JUAN RADA ${ }^{1}$
}

\begin{abstract}
We find the extremal graphs for the exponential of well known vertexdegree-based topological indices over $\mathcal{G}_{n}$, the set of graphs with $n$ non-isolated vertices.
\end{abstract}

\section{INTRODUCTION}

A topological index is a number associated to a graph (for motivation and chemical applications see $[2,10,11,16,17])$. One important class of topological indices are the so-called vertex-degree-based (VDB for short) topological indices, which strongly depend on the degree of the vertices of the graph $[1,3,6,8,9,12]$.

More precisely, let $\mathcal{G}_{n}$ be the set of graphs with $n$ non-isolated vertices. Consider the function $m: \mathcal{G}_{n} \rightarrow \mathbb{R}^{\frac{(n-1) n}{2}}$ defined as $m(G)=\left(m_{i j}(G)\right)_{(i, j) \in K}$ for every $G \in \mathcal{G}_{n}$, where

$$
K=\{(i, j) \in \mathbb{N} \times \mathbb{N}: 1 \leq i \leq j \leq n-1\}
$$

and $m_{i j}(G)$ is the number of edges in $G$ joining vertices of degree $i$ and $j$. We order $K$ lexicographically so that $m(G)$ is a vector of $\mathbb{R}^{\frac{(n-1) n}{2}}$, for each $G \in \mathcal{G}_{n}$. A VDB topological index over $\mathcal{G}_{n}$ is a function $\varphi: \mathcal{G}_{n} \rightarrow \mathbb{R}$ induced by a vector $\varphi=\left(\varphi_{i j}\right)_{(i, j) \in K} \in \mathbb{R}^{\frac{(n-1) n}{2}}$, defined as

$$
\varphi(G)=m(G) \cdot \varphi
$$

the dot product of $m(G)$ and $\varphi$ as vectors in $\mathbb{R}^{\frac{(n-1) n}{2}}[13]$. In other words,

$$
\varphi(G)=\sum_{(i, j) \in K} m_{i j}(G) \varphi_{i j}
$$

Key words and phrases. Vertex-degree-based topological indices, exponential topological indices, extremal graphs.

2010 Mathematics Subject Classification. Primary:05C35. Secondary: 05C07.

DOI 10.46793/KgJMat2201.105C

Received: March 12, 2019.

Accepted: September 03, 2019. 
for every $G \in \mathcal{G}_{n}$.

The best known VDB topological indices are the following:

(a) the First Zagreb index [7], denoted by $\mathcal{F} Z$ and defined as $\varphi_{i j}=i+j$ for all $(i, j) \in K$

(b) the Second Zagreb index [7], denoted by $\mathcal{S} Z$ and defined as $\varphi_{i j}=i j$;

(c) the Randić index [15], denoted by $\chi$ and defined as $\varphi_{i j}=\frac{1}{\sqrt{i j}}$;

(d) the Harmonic index [20], denoted by $\mathcal{H}$ and defined as $\varphi_{i j}=\frac{2}{i+j}$;

(e) the Geometric-Arithmetic index [18], denoted by $\mathcal{G A}$ and defined as $\varphi_{i j}=\frac{2 \sqrt{i j}}{i+j}$;

(f) the Sum-Connectivity index [19], denoted by $\mathcal{S} \mathcal{C}$ and defined as $\varphi_{i j}=\frac{1}{\sqrt{i+j}}$;

(g) the Atom-Bond-Connectivity index [4], denoted by $\mathcal{A B C}$ and defined as $\varphi_{i j}=$ $\sqrt{\frac{i+j-2}{i j}}$

(h) the Augmented Zagreb index [5], denoted by $\mathcal{A} z$ and defined as $\varphi_{i j}=\left(\frac{i j}{i+j-2}\right)^{3}$.

In a recent paper [14], the exponential of a VDB topological index $\varphi=\left(\varphi_{i j}\right)_{(i, j) \in K}$ was introduced as $\psi=e^{\varphi} \in \mathbb{R}^{\frac{(n-1) n}{2}}$, defined as

$$
\psi_{i j}=e^{\varphi_{i j}}
$$

for all $(i, j) \in K$. Among other things, it was shown in [14] that the exponential VDB topological indices have good discrimination properties. In this paper we determine the extremal graphs for the exponentials of all the best known VDB topological indices listed above, over the set $\mathcal{G}_{n}$.

Consider the VDB topological index $\varphi=\left(\varphi_{i j}\right)_{(i, j) \in K} \in \mathbb{R}^{\frac{(n-1) n}{2}}$. The vector $\varphi$ can be viewed as a function $\varphi: K \rightarrow \mathbb{R}$, where $\varphi(x, y)=\varphi_{x y}$ for all $(x, y) \in K$. We define the auxiliary function $f_{\varphi}: K \rightarrow \mathbb{R}$ defined as $f_{\varphi}(x, y)=\frac{x y \varphi_{x y}}{x+y}$. In order to find the maximal and minimal values of $\varphi$ over $\mathcal{G}_{n}$, it is sufficiently to find the maximal and minimal values of $f_{\varphi}$ over $K[12]$. Recall that

$$
K_{\min }\left(f_{\varphi}\right)=\left\{(r, s) \in K: f_{\varphi}(r, s)=\min _{(i, j) \in K} f_{\varphi}(i, j)\right\}
$$

and

$$
K_{\max }\left(f_{\varphi}\right)=\left\{(p, q) \in K: f_{\varphi}(p, q)=\max _{(i, j) \in K} f_{\varphi}(i, j)\right\} .
$$

We use notations $K_{\min }^{c}\left(f_{\varphi}\right)=K-K_{\min }\left(f_{\varphi}\right)$ and $K_{\max }^{c}\left(f_{\varphi}\right)=K-K_{\max }\left(f_{\varphi}\right)$. In order to compute $K_{\min }\left(f_{\varphi}\right)$ and $K_{\max }\left(f_{\varphi}\right)$, we will assume that $\varphi$ is a real continuous and differentiable function defined over the compact set

$$
\widehat{K}=\{(x, y) \in \mathbb{R} \times \mathbb{R}: 1 \leq x \leq y \leq n-1\} .
$$

Hence, $f_{\varphi}: \widehat{K} \rightarrow \mathbb{R}$ defined as $f_{\varphi}(x, y)=\frac{x y \varphi_{x y}}{x+y}$ for all $(x, y) \in \widehat{K}$, is also continuous and differentiable over $\widehat{K}$. 


\section{Extremal Values of Exponentials of VDB Topological Indices}

Based on [12, Theorem 2.3] and [12, Theorem 2.7] we will compute the maximal and minimal values of the exponentials of the VDB topological indices listed in the Introduction.

Theorem 2.1. Let $e^{\mathcal{F Z}}$ be the exponential of the first Zagreb index $\mathcal{F}$. Then:

1. $K_{n}$ is the unique maximal graph over $\mathcal{G}_{n}$ with respect to $e^{\mathfrak{F Z}}$, with value $\frac{n-1}{2} n e^{2(n-1)}$;

2. if $n$ is even (resp. odd), $\frac{n}{2} K_{2}$ (resp. $\frac{n-3}{2} K_{2} \cup P_{3}$ ) is the unique minimal graph over $\mathcal{G}_{n}$ with respect to $e^{\mathcal{F} z}$, with value $\frac{n}{2} e^{2}$ (resp. $\left.\frac{n-3}{2} e^{2}+2 e^{3}\right)$.

Proof. The associated function for $e^{\mathfrak{F z}}$ over $\widehat{K}$ is

$$
f_{e^{\mathcal{F Z}}}(x, y)=\frac{x y e^{x+y}}{x+y} .
$$

Note that

$$
\frac{\partial}{\partial x}\left(\frac{x y e^{x+y}}{x+y}\right)=y \frac{e^{x+y}}{(x+y)^{2}}\left(x^{2}+y x+y\right)>0,
$$

for all $(x, y) \in \widehat{K}$.

1. By (2.1), the greatest value of $f_{e^{\mathcal{F}}}$ over $\widehat{K}$ is attained in the diagonal

$$
D=\{(x, y) \in \widehat{K}: y=x\} \text {. }
$$

Note that

$$
\left(f_{e^{\mathcal{F Z}}}(x, x)\right)^{\prime}=\left(\frac{1}{2} x e^{2 x}\right)^{\prime}=\frac{1}{2} e^{2 x}(2 x+1)>0,
$$

for all $x \in[1, n-1]$. It follows that

$$
K_{\max }\left(f_{e^{\mathcal{F}}}\right)=\{(n-1, n-1)\} .
$$

Now we apply [12, Theorem 2.3] to obtain

$$
e^{\mathcal{F Z}}(G) \leq n f_{e^{\mathcal{F Z}}}(n-1, n-1)=\frac{1}{2}(n-1) n e^{2(n-1)}=e^{\mathcal{F Z}}\left(K_{n}\right) .
$$

Moreover, equality is obtained if and only if $m_{r s}(G)=0$ for all $(r, s) \neq(n-1, n-1)$. This is precisely $G=K_{n}$.

2. By $(2.1)$, the minimal value of $f_{e^{\mathfrak{F}}}$ over $\widehat{K}$ is attained in the vertical line

$$
V=\{(x, y) \in \widehat{K}: x=1\}
$$

Note that

$$
\left(f_{e^{\mathfrak{F Z}}}(1, y)\right)^{\prime}=\left(y \frac{e^{y+1}}{y+1}\right)^{\prime}=\frac{e^{y+1}}{(y+1)^{2}}\left(y^{2}+y+1\right)>0
$$


for all $y \in[1, n-1]$. Hence,

$$
K_{\min }\left(f_{e^{\mathfrak{F} z}}\right)=\{(1,1)\} .
$$

It follows from [12, Theorem 2.3] that if $n$ is even, then

$$
e^{\mathfrak{F Z}}(G) \geq n f_{e^{\mathfrak{F Z}}}(1,1)=\frac{n}{2} e^{2}=e^{\mathfrak{F Z}}\left(\frac{n}{2} K_{2}\right),
$$

for all $G \in \mathcal{G}_{n}$. Furthermore, equality is obtained if and only if $m_{r s}(G)=0$ for all $(r, s) \neq(1,1)$. This is precisely $G=\frac{n}{2} K_{2}$.

Finally, assume that $n$ is odd. From (2.1) and (2.2) we deduce that

$$
f_{e^{\mathfrak{F Z}}}(1,2)<f_{e^{\mathfrak{F Z}}}(x, y),
$$

for all $(x, y) \in K$ different from $(1,1)$ and $(1,2)$. Hence, by [12, Theorem 2.7],

$$
\begin{aligned}
e^{\mathfrak{F Z}}(G) & \geq f_{e^{\mathfrak{F Z}}}(1,1)(n-3)+3 f_{e^{\mathcal{F Z}}}(1,2) \\
& =\frac{n-3}{2} e^{2}+2 e^{3} \\
& =e^{\mathfrak{F Z}}\left(\frac{n-3}{2} K_{2} \cup P_{3}\right),
\end{aligned}
$$

for all $G \in \mathcal{G}_{n}$. Equality occurs if and only if $G=\frac{n-3}{2} K_{2} \cup P_{3}$.

An identical argument as in the proof of Theorem 2.1 works for the exponential of the Second Zagreb index $\mathcal{S Z}$ and the Atom-Bond-Connectivity index $\mathcal{A B C}$. We state them without proof.

Theorem 2.2. Let $e^{\text {Sz }}$ be the exponential of the Second Zagreb index Sz. Then:

(a) $K_{n}$ is the unique maximal graph over $\mathcal{G}_{n}$ with respect to $e^{\delta z}$, with value

$$
\frac{1}{2}(n-1) n e^{(n-1)^{2}}
$$

(b) if $n$ is even (resp. odd), $\frac{n}{2} K_{2}$ (resp. $\left.\frac{n-3}{2} K_{2} \cup P_{3}\right)$ is the unique minimal graph over $\mathcal{G}_{n}$ with respect to $e^{\mathcal{\delta} z}$, with value $\frac{n}{2}$ e (resp. $\left.\frac{n-3}{2} e+2 e^{2}\right)$.

Theorem 2.3. Let $e^{\mathcal{A B C}}$ be the exponential of the $\mathcal{A B C}$ index. Then:

(a) $K_{n}$ is the unique maximal graph over $\mathcal{G}_{n}$ with respect to $e^{\mathcal{A B C}}$, with value

$$
\frac{1}{2}(n-1) n e^{\frac{1}{n-1} \sqrt{2(n-2)}}
$$

(b) if $n$ is even (resp. odd), $\frac{n}{2} K_{2}$ (resp. $\left.\frac{n-3}{2} K_{2} \cup P_{3}\right)$ is the unique minimal graph over $\mathcal{G}_{n}$ with respect to $e^{\mathcal{A B C}}$, with value $\frac{n}{2}$ (resp. $\left.\frac{n-3}{2}+2 e^{\frac{1}{\sqrt{2}}}\right)$.

We next examine the exponential of the Harmonic index.

Theorem 2.4. Let $e^{\mathcal{H}}$ be the exponential of the Harmonic index $\mathcal{H}$. Then:

1. $K_{n}$ is the unique maximal graph over $\mathcal{G}_{n}$ with respect to $e^{\mathcal{H}}$, with value

$$
\frac{1}{2}(n-1) n e^{\frac{1}{n-1}}
$$


2. $S_{n}$ is the unique minimal graph over $\mathcal{G}_{n}$ with respect to $e^{\mathcal{H}}$, with value $(n-1) e^{\frac{2}{n}}$. Proof. The associated function for $e^{\mathcal{H}}$ is

$$
f_{e^{\mathcal{H}}}(x, y)=\frac{x y e^{\frac{2}{x+y}}}{x+y} .
$$

Now

$$
\frac{\partial}{\partial x}\left(\frac{x y e^{\frac{2}{x+y}}}{x+y}\right)=y e^{\frac{2}{x+y}} \frac{-2 x+x y+y^{2}}{(x+y)^{3}}>0,
$$

for all $(x, y) \in \widehat{K}-\{(1,1)\}$ (in $(1,1)$ equals to zero).

1. By (2.3), the maximal value of $f_{e^{\mathscr{H}}}$ over $\widehat{K}$ is attained in the diagonal

$$
D=\{(x, y) \in \widehat{K}: y=x\} \text {. }
$$

Note that

$$
\left(f_{e^{\mathscr{H}}}(x, x)\right)^{\prime}=\left(\frac{1}{2} x e^{\frac{1}{x}}\right)^{\prime}=\frac{1}{2 x} e^{\frac{1}{x}}(x-1)>0,
$$

for all $x \in(1, n-1]$. Hence,

$$
K_{\max }\left(f_{e^{\mathcal{H}}}\right)=\{(n-1, n-1)\} .
$$

Now we apply [12, Theorem 2.3] to obtain

$$
e^{\mathcal{H}}(G) \leq n f_{e^{\mathcal{H}}}(n-1, n-1)=\frac{1}{2}(n-1) n e^{\frac{1}{n-1}}=e^{\mathcal{H}}\left(K_{n}\right) .
$$

Moreover, equality holds if and only if $m_{r s}(G)=0$ for all $(r, s) \neq(n-1, n-1)$, i.e., $G=K_{n}$.

2. By (2.3), the minimal value of $f_{e^{\mathscr{H}}}$ over $\widehat{K}$ is attained in the vertical line

$$
V=\{(x, y) \in \widehat{K}: x=1\} \text {. }
$$

Note that

$$
\left(f_{e^{\mathcal{H}}}(1, y)\right)^{\prime}=\left(y \frac{e^{\frac{2}{y+1}}}{y+1}\right)^{\prime}=-e^{\frac{2}{y+1}} \frac{y-1}{(y+1)^{3}}<0,
$$

for all $y \in(1, n-1]$. It follows that,

$$
K_{\min }\left(f_{e^{\mathcal{H}}}\right)=\{(1, n-1)\}
$$

Now, by [12, Theorem 2.3],

$$
e^{\mathcal{H}}(G) \geq n f_{e^{\mathcal{H}}}(1, n-1)=(n-1) e^{\frac{2}{n}}=e^{\mathcal{H}}\left(S_{n}\right),
$$

for all $G \in \mathcal{G}_{n}$. Equality holds if and only if $m_{r s}(G)=0$ for all $(r, s) \neq(1, n-1)$, i.e., $G=S_{n}$. 
The extremal values of the exponential of the Randic index $\chi$ and the AugmentedZagreb $\mathcal{A} z$ can also be computed using an identical argument as in the proof of Theorem 2.4. We state them without proof.

Theorem 2.5. ([14, Theorem 4.3]). Let $e^{\chi}$ be the exponential of the Randic index $\chi$. Then:

(a) $K_{n}$ is the unique maximal graph over $\mathcal{G}_{n}$ with respect to $e^{\chi}$, with value

$$
\frac{1}{2}(n-1) n e^{\frac{1}{n-1}}
$$

(b) $S_{n}$ is the unique minimal graph over $\mathcal{G}_{n}$ with respect to $e^{\chi}$, with value

$$
(n-1) e^{\frac{1}{\sqrt{n-1}}}
$$

Theorem 2.6. Let $e^{\mathcal{A Z}}$ be the exponential of the Augmented-Zagreb index AZ. Then:

(a) $K_{n}$ is the unique maximal graph over $\mathcal{G}_{n}$ with respect to $e^{\mathcal{A z}}$, with value

$$
\frac{1}{2}(n-1) n e^{\left(\frac{(n-1)^{2}}{2(n-2)}\right)^{3}}
$$

(b) $S_{n}$ is the unique minimal graph over $\mathcal{G}_{n}$ with respect to $e^{\mathcal{A z}}$, with value

$$
(n-1) e^{\left(\frac{n-1}{n-2}\right)^{3}}
$$

Next we consider the exponential of the Geometric-Arithmetic index $\mathcal{G A}$.

Theorem 2.7. Let $e^{\mathcal{G A}}$ be the exponential of the Geometric-Arithmetic index $\mathcal{G A}$. Then

(a) $K_{n}$ is the unique maximal graph over $\mathcal{G}_{n}$ with respect to $e^{\mathcal{G A}}$, with value $\frac{1}{2}(n-1) n e ;$

(b) If $n \leq 34$ is even (resp. odd), $\frac{n}{2} K_{2}$ (resp. $\left.\frac{n-3}{2} K_{2} \cup P_{3}\right)$ is the unique minimal graph over $\mathcal{G}_{n}$ with respect to $e^{\mathcal{G A}}$ with value $\frac{n}{2} e\left(\right.$ resp. $\left.\frac{n-3}{2} e+2 e^{\frac{2 \sqrt{2}}{3}}\right)$;

(c) If $n \geq 35$, then $S_{n}$ is the unique minimal graph over $\mathcal{G}_{n}$ with respect to $e^{\mathcal{G A}}$, with value $(n-1) e^{\frac{2}{n} \sqrt{n-1}}$.

Proof. The associated function for $e^{\mathcal{G A}}$ is

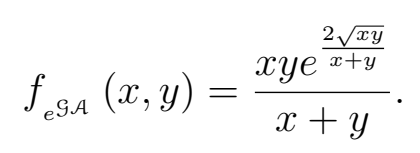

Note that

$$
\frac{\partial}{\partial x}\left(\frac{x y e^{\frac{2 \sqrt{x y}}{x+y}}}{x+y}\right)=y^{2} e^{2 \frac{\sqrt{x y}}{x+y}} \frac{\sqrt{x y}(x+y+\sqrt{x y})-x^{2}}{\sqrt{x y}(x+y)^{3}}>0,
$$

for all $(x, y) \in \widehat{K}$.

(a) By (2.4), the maximal value of $f_{e \mathcal{A A}}$ over $\widehat{K}$ is attained in the diagonal

$$
D=\{(x, y) \in \widehat{K}: y=x\} \text {. }
$$


Note that

$$
\left(f_{e \mathcal{A}}(x, x)\right)^{\prime}=\left(\frac{1}{2} e x\right)^{\prime}=\frac{1}{2} e>0,
$$

for all $x \in[1, n-1]$. Hence,

$$
K_{\max }\left(f_{e^{\mathcal{G}}}\right)=\{(n-1, n-1)\} .
$$

It follows from [12, Theorem 2.3] that

$$
e^{\mathcal{G A}}(G) \leq n f_{e^{\mathcal{S A}}}(n-1, n-1)=\frac{1}{2}(n-1) n e,
$$

for all $G \in \mathcal{G}_{n}$. Equality holds if and only if $m_{r s}(G)=0$ for all $(r, s) \neq(n-1, n-1)$, i.e., $G=K_{n}$.

(b) By (2.4), the minimal value of $f_{e \mathcal{G A}}$ over $\widehat{K}$ is attained in the vertical line

$$
V=\{(x, y) \in \widehat{K}: x=1\} .
$$

The graph of the one variable function $f_{e^{\mathcal{A}}}(1, y)=\frac{y}{y+1} e^{\frac{2 \sqrt{y}}{y+1}}$ is shown in Figure 1 .

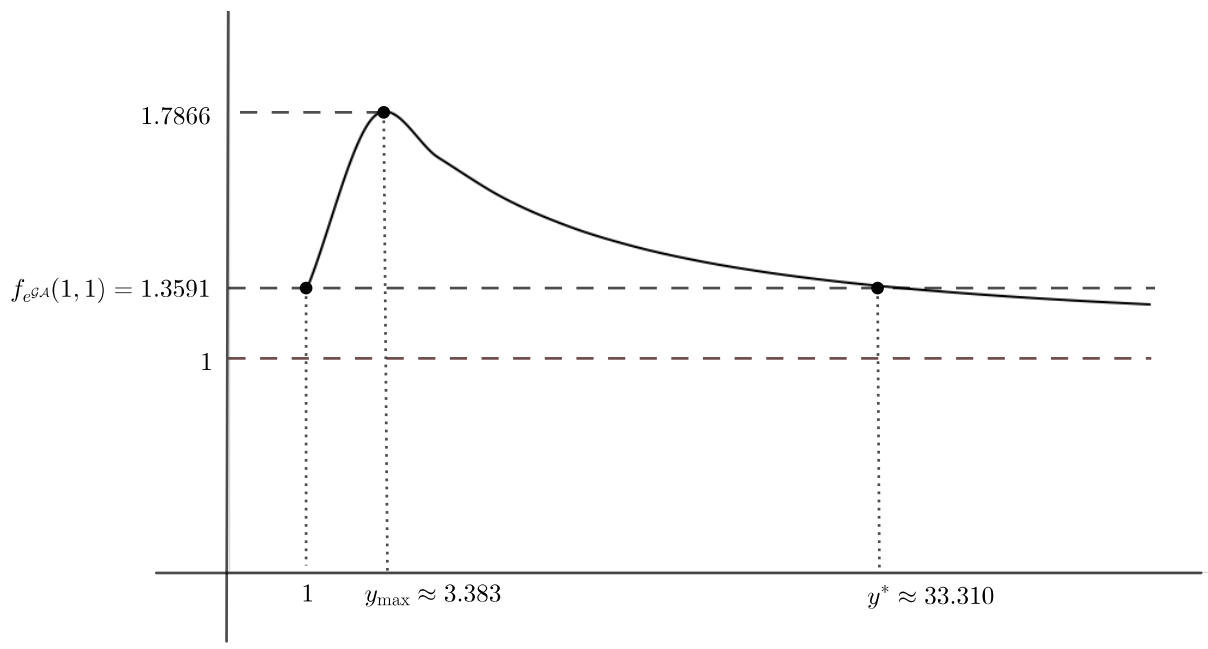

FIGURE 1. Graph of $f_{e^{\mathcal{A}}}(1, y)$.

The function $f_{e \mathcal{S A}_{\mathcal{A}}}(1, y)$ attains its maximal value at $y_{\max } \approx 3.383$, is strictly decreasing for $y>y_{\max }, f_{e \mathcal{G A}_{\mathcal{A}}}\left(1, y^{*}\right)=f_{e^{\mathcal{S}_{\mathcal{A}}}}(1,1)=1.3591$ for $y^{*} \approx 33.310$ and

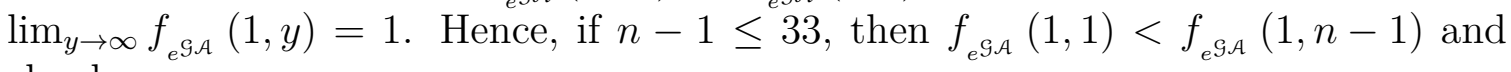
clearly

$$
K_{\min }\left(f_{e^{\mathcal{S}} \mathcal{A}}\right)=\{(1,1)\}
$$


A similar argument as in the proof of Theorem 2.1 shows that if $n \leq 34$ is even (resp. odd), then the minimal value of $e^{\mathcal{G A}}$ over $\mathcal{G}_{n}$ is attained uniquely in $\frac{n}{2} K_{2}$ (resp. $\left.\frac{n-3}{2} K_{2} \cup P_{3}\right)$ with value $\frac{n}{2} e$ (resp. $\frac{n-3}{2} e+2 e^{\frac{2 \sqrt{2}}{3}}$ ). On the other hand, if $n-1 \geq 34$, then

$$
K_{\min }\left(f_{e \mathcal{S A}}\right)=\{(1, n-1)\} .
$$

Now, by [12, Theorem 2.3],

$$
e^{\mathcal{G A}}(G) \geq n f_{e^{\mathcal{A}}}(1, n-1)=(n-1) e^{\frac{2}{n} \sqrt{n-1}}=e^{\mathcal{H}}\left(S_{n}\right),
$$

for all $G \in \mathcal{G}_{n}$. Equality holds if and only if $m_{r s}(G)=0$ for all $(r, s) \neq(1, n-1)$, i.e., $G=S_{n}$.

A very similar argument to the one used in the proof of Theorem 2.7, gives the extremal values for the exponential of the Sum-Connectivity index SC. In the case of the minimal value of $e^{\mathcal{S E}}$ over $\widehat{K}$, the one variable function $f_{e^{s e}}(1, y)=\frac{y}{y+1} e^{\frac{1}{\sqrt{y+1}}}$, behaves similarly to the function $f_{e \mathcal{A}}(1, y)$ for $y \geq 1$. It attains its maximal value at $y_{\max } \approx 4.8284$, is strictly decreasing for $y>y_{\max }, f_{e^{8 \mathcal{C}}}\left(1, y^{*}\right)=f_{e^{\text {se }}}(1,1)=1.0141$ for $y^{*} \approx 4986,3$ and $\lim _{y \rightarrow \infty} f_{e \text { se }}(1, y)=1$. Hence, if $n-1 \leq 4986$, then $f_{e^{\text {se }}}(1,1)<$ $f_{\text {ese }}(1, n-1)$ and if $n-1 \geq 4987$, then $f_{e s e}(1, n-1)<f_{e s e}(1,1)$. We state it without proof.

Theorem 2.8. Let $e^{\text {se }}$ be the exponential of the Sum-Connectivity index SC. Then:

(a) $K_{n}$ is the unique maximal graph over $\mathcal{G}_{n}$ with respect to $e^{\text {se }}$, with value

$$
\frac{1}{2}(n-1) n e^{\frac{1}{\sqrt{2(n-1)}}}
$$

(b) if $n \leq 4987$ is even (resp. odd), $\frac{n}{2} K_{2}$ (resp. $\frac{n-3}{2} K_{2} \cup P_{3}$ ) is the unique minimal graph over $\mathcal{G}_{n}$ with respect to $e^{\text {se }}$ with value $\frac{n}{2} e^{\frac{1}{\sqrt{2}}}$ (resp. $\left.\frac{n-3}{2} e^{\frac{1}{\sqrt{2}}}+2 e^{\frac{1}{\sqrt{3}}}\right)$;

(c) if $n \geq 4988$, then $S_{n}$ is the unique minimal graph over $\mathcal{G}_{n}$ with respect to $e^{\text {se }}$, with value $(n-1) e^{\frac{1}{\sqrt{n}}}$.

\section{REFERENCES}

[1] R. Cruz, T. Pérez and J. Rada, Extremal values of vertex-degree-based topological indices over graphs, J. Appl. Math. Comput. 48 (2015), 395-406.

[2] J. Devillers and A. T. Balaban, Topological Indices and Related Descriptors, in: QSAR and QSPR, Gordon \& Breach, Amsterdam, 1999.

[3] T. Došlić, B. Furtula, A. Graovac, I. Gutman, S. Moradi and Z. Yarahmadi, On vertex-degreebased molecular structure descriptors, MATCH Commun. Math. Comput. Chem. 66 (2011), 613-626.

[4] E. Estrada, L. Torres, L. Rodríguez and I. Gutman, An atom-bond connectivity index: Modelling the enthalpy of formation of alkanes, Indian Journal of Chemistry 37A (1998), 849-855.

[5] B. Furtula, A. Graovac and D. Vukičević, Augmented Zagreb index, J. Math. Chem. 48 (2010), 370-380.

[6] B. Furtula, I. Gutman and M. Dehmer, On structure-sensitivity of degree-based topological indices, Appl. Math. Comput. 219 (2013), 8973-8978. 
[7] I. Gutman and N. Trinajstić, Graph theory and molecular orbitals. Total $\pi$-electron energy of alternant hydrocarbons, Chemical Physics Letters 17 (1972), 535-538.

[8] I. Gutman and J. Tošović, Testing the quality of molecular structure descriptors. Vertex-degreebased topological indices, Journal of the Serbian Chemical Society 78 (2013), 805-810.

[9] I. Gutman, Degree-based topological indices, Croatica Chemica Acta 86 (2013), 351-361.

[10] L. Kier and L. Hall, Molecular Connectivity in Chemistry and Drug Research, Academic Press, New York, 1976.

[11] L. Kier and L. Hall, Molecular Connectivity in Structure-Activity Analysis, Wiley, New York, 1986.

[12] J. Rada and R. Cruz, Vertex-degree-based topological indices over graphs, MATCH Commun. Math. Comput. Chem. 72 (2014), 603-616.

[13] J. Rada and S. Bermudo, Is every graph the extremal value of a vertex-degree-based topological index?, MATCH Commun. Math. Comput. Chem. 81 (2019), 315-323.

[14] J. Rada, Exponential vertex-degree-based topological indices and discrimination, MATCH Commun. Math. Comput. Chem. 82 (2019), 29-41.

[15] M. Randić, On characterization of molecular branching, Journal of the American Chemical Society 97 (1975), 6609-6615.

[16] R. Todeschini and V. Consonni, Handbook of Molecular Descriptors, Wiley-VCH, Weinheim, 2000.

[17] R. Todeschini and V. Consonni, Molecular Descriptors for Chemoinformatics, Wiley-VCH, Weinheim, 2009.

[18] D. Vukičević and B. Furtula, Topological index based on the ratios of geometrical and arithmetical means of end-vertex degrees of edges, J. Math. Chem. 46 (2009), 1369-1376.

[19] L. Zhong, The harmonic index for graphs, Appl. Math. Lett. 25 (2012), 561-566.

[20] B. Zhou and N. Trinajstić, On a novel connectivity index, J. Math. Chem. 46 (2009), 1252-1270.

\author{
${ }^{1}$ Instituto de Matematicas, \\ UniversidAd DE ANTIOQUiA, \\ Medellín, Colombia \\ Email address: roberto.cruz@udea.edu.co \\ Email address: pablo.rada@udea.edu.co
}



Kragujevac Journal of Mathematics

Volume 46(1) (2022), Pages 115-124.

\title{
DIFFERENTIAL SUBORDINATION RESULTS FOR HOLOMORPHIC FUNCTIONS RELATED TO GENERALIZED DIFFERENTIAL OPERATOR
}

\author{
ABBAS KAREEM WANAS ${ }^{1}$
}

\begin{abstract}
In the present investigation, we use the principle of subordination to introduce a new family for holomorphic functions defined by generalized differential operator. Also we establish some interesting geometric properties for functions belonging to this family.
\end{abstract}

\section{Introduction AND PRELIMINARIES}

Let $\mathcal{A}_{m}$ stands for the family of functions $f$ of the form:

$$
f(z)=z+\sum_{n=m+1}^{\infty} a_{n} z^{n} \quad(m \in \mathbb{N}=\{1,2, \ldots\}, z \in U),
$$

which are holomorphic in the open unit disk $U=\{z \in C:|z|<1\}$.

For two functions $f$ and $g$ holomorphic in $U$, we say that the function $f$ is subordinate to $g$, written $f \prec g$ or $f(z) \prec g(z)(z \in U)$, if there exists a Schwarz function $w$ holomorphic in $U$ with $w(0)=0$ and $|w(z)|<1, z \in U$, such that $f(z)=g(w(z))$, $z \in U$. In particular, if the function $g$ is univalent in $U$, then $f \prec g$ if and only if $f(0)=g(0)$ and $f(U) \subset g(U)$ (see [6]).

If $f \in \mathcal{A}_{m}$ is given by (1.1) and $g \in \mathcal{A}_{m}$ given by

$$
g(z)=z+\sum_{n=m+1}^{\infty} b_{n} z^{n} \quad(m \in \mathbb{N}=\{1,2, \ldots\}, z \in U),
$$

Key words and phrases. Holomorphic functions, Differential subordination, Convex univalent, Hadamard product, Generalized differential operator.

2010 Mathematics Subject Classification. Primary: 30C45. Secondary: 30A20.

DOI 10.46793/KgJMat2201.115W

Received: March 29, 2019.

Accepted: September 06, 2019. 
then the Hadamard product (or convolution) $f * g$ of $f$ and $g$ is defined by

$$
(f * g)(z)=z+\sum_{n=m+1}^{\infty} a_{n} b_{n} z^{n}=(g * f)(z) .
$$

A function $f \in \mathcal{A}_{m}$ is said to be starlike of order $\rho$ in $U$ if

$$
\operatorname{Re}\left\{\frac{z f^{\prime}(z)}{f(z)}\right\}>\rho \quad(0 \leq \rho<1, z \in U) .
$$

Indicate the class of all starlike functions of order $\rho$ in $U$ by $S^{*}(\rho)$.

A function $f \in \mathcal{A}_{m}$ is said to be prestarlike of order $\rho$ in $U$ if

$$
\frac{z}{(1-z)^{2(1-\rho)}} * f(z) \in S^{*}(\rho) \quad(\rho<1)
$$

Indicate the class of all prestarlike functions of order $\rho$ in $U$ by $\operatorname{Re}(\rho)$.

Clearly a function $f \in \mathcal{A}_{m}$ is in the class $\operatorname{Re}(0)$ if and only if $f$ is convex univalent in $U$ and $\operatorname{Re}\left(\frac{1}{2}\right)=S^{*}\left(\frac{1}{2}\right)$.

For $\sigma \in N_{0}=N \cup\{0\}, \alpha, \delta \geq 0, \tau, \lambda, \beta>0$ and $\alpha \neq \lambda$, we consider the generalized differential operator $A_{\tau, \lambda, \delta}^{\sigma}(\alpha, \beta): \mathcal{A}_{m} \rightarrow \mathcal{A}_{m}$, introduced by Amourah and Darus [2], where

$$
A_{\tau, \lambda, \delta}^{\sigma}(\alpha, \beta) f(z)=z+\sum_{n=m+1}^{\infty}\left[1+\frac{(n-1)((\lambda-\alpha) \beta+n \delta)}{\tau+\lambda}\right]^{\sigma} a_{n} z^{n} .
$$

It is readily verified from (1.2) that

$$
\begin{aligned}
z\left(A_{\tau, \lambda, \delta}^{\sigma}(\alpha, \beta) f(z)\right)^{\prime}= & \frac{\tau+\lambda}{(\lambda-\alpha) \beta+n \delta} A_{\tau, \lambda, \delta}^{\sigma+1}(\alpha, \beta) f(z) \\
& +\left(1-\frac{\tau+\lambda}{(\lambda-\alpha) \beta+n \delta}\right) A_{\tau, \lambda, \delta}^{\sigma}(\alpha, \beta) f(z) .
\end{aligned}
$$

Here, we would point out some of the special cases of the operator defined by (1.2) can be found in $[1,4,5,11]$.

Let $H$ be the class of functions $h$ with $h(0)=1$, which are holomorphic and convex univalent in $U$.

Definition 1.1. A function $f \in \mathcal{A}_{m}$ is said to be in the class $\mathcal{M}(\eta, \sigma, \tau, \lambda, \delta, \alpha, \beta, m ; h)$ if it satisfies the subordination condition:

$$
\frac{1}{z}\left[\left(1-\frac{\eta(\tau+\lambda)}{(\lambda-\alpha) \beta+n \delta}\right) A_{\tau, \lambda, \delta}^{\sigma}(\alpha, \beta) f(z)+\frac{\eta(\tau+\lambda)}{(\lambda-\alpha) \beta+n \delta} A_{\tau, \lambda, \delta}^{\sigma+1}(\alpha, \beta) f(z)\right] \prec h(z),
$$

where $\eta \in C, \sigma \in N_{0}=N \cup\{0\}, \alpha, \delta \geq 0, \tau, \lambda, \beta>0, \alpha \neq \lambda$ and $h \in H$.

Now, we need the following lemmas that will be used to prove our main results. 
Lemma $1.1([8])$. Let $g$ be holomorphic in $U$ and let $h$ be holomorphic and convex univalent in $U$ with $h(0)=g(0)$. If

$$
g(z)+\frac{1}{\mu} z g^{\prime}(z) \prec h(z),
$$

where $\operatorname{Re}(\mu) \geq 0$ and $\mu \neq 0$, then

$$
g(z) \prec \breve{h}(z)=\mu z^{-\mu} \int_{0}^{z} t^{\mu-1} h(t) d t \prec h(z)
$$

and $\breve{h}$ is the best dominant of (1.5).

Lemma $1.2([10])$. Let $\rho<1, f \in S^{*}(\rho)$ and $g \in \operatorname{Re}(\rho)$. Then, for any holomorphic function $F$ in $U$

$$
\frac{g *(f F)}{g * f}(U) \subset \overline{c o}(F(U)),
$$

where $\overline{c o}(F(U))$ denotes the closed convex hull of $F(U)$.

Such type of study was carried out by various authors for another classes, like, Liu [7], Prajapat and Raina [9], Atshan and Wanas [3], Wanas [12] and Wanas and Majeed [13].

\section{Main Results}

Theorem 2.1. Let $0 \leq \eta<\varepsilon$. Then

$$
\mathcal{M}(\varepsilon, \sigma, \tau, \lambda, \delta, \alpha, \beta, m ; h) \subset \mathcal{M}(\eta, \sigma, \tau, \lambda, \delta, \alpha, \beta, m ; h) .
$$

Proof. Let $0 \leq \eta<\varepsilon$ and $f \in \mathcal{M}(\varepsilon, \sigma, \tau, \lambda, \delta, \alpha, \beta, m ; h)$. Assume that

$$
g(z)=\frac{A_{\tau, \lambda, \delta}^{\sigma}(\alpha, \beta) f(z)}{z}=1+\sum_{n=m+1}^{\infty}\left[1+\frac{(n-1)((\lambda-\alpha) \beta+n \delta)}{\tau+\lambda}\right]^{\sigma} a_{n} z^{n-1} .
$$

It is obvious that the function $g$ is holomorphic in $U$ and $g(0)=1$. Since $f \in$ $\mathcal{M}(\varepsilon, \sigma, \tau, \lambda, \delta, \alpha, \beta, m ; h)$, then we deduce that

$$
\frac{1}{z}\left[\left(1-\frac{\varepsilon(\tau+\lambda)}{(\lambda-\alpha) \beta+n \delta}\right) A_{\tau, \lambda, \delta}^{\sigma}(\alpha, \beta) f(z)+\frac{\varepsilon(\tau+\lambda)}{(\lambda-\alpha) \beta+n \delta} A_{\tau, \lambda, \delta}^{\sigma+1}(\alpha, \beta) f(z)\right] \prec h(z) .
$$

Differentiating both sides of (2.1) with respect to $z$ and using (1.3) and (2.2), we find that

$$
\begin{aligned}
& \frac{1}{z}\left[\left(1-\frac{\varepsilon(\tau+\lambda)}{(\lambda-\alpha) \beta+n \delta}\right) A_{\tau, \lambda, \delta}^{\sigma}(\alpha, \beta) f(z)+\frac{\varepsilon(\tau+\lambda)}{(\lambda-\alpha) \beta+n \delta} A_{\tau, \lambda, \delta}^{\sigma+1}(\alpha, \beta) f(z)\right] \\
= & \frac{1}{z}\left[(1-\varepsilon) A_{\tau, \lambda, \delta}^{\sigma}(\alpha, \beta) f(z)+\varepsilon\left(1-\frac{\tau+\lambda}{(\lambda-\alpha) \beta+n \delta}\right) A_{\tau, \lambda, \delta}^{\sigma}(\alpha, \beta) f(z)\right. \\
& \left.+\frac{\varepsilon(\tau+\lambda)}{(\lambda-\alpha) \beta+n \delta} A_{\tau, \lambda, \delta}^{\sigma+1}(\alpha, \beta) f(z)\right]
\end{aligned}
$$




$$
\begin{aligned}
& =(1-\varepsilon) \frac{A_{\tau, \lambda, \delta}^{\sigma}(\alpha, \beta) f(z)}{z}+\varepsilon\left(A_{\tau, \lambda, \delta}^{\sigma}(\alpha, \beta) f(z)\right)^{\prime} \\
& =\frac{A_{\tau, \lambda, \delta}^{\sigma}(\alpha, \beta) f(z)}{z}+\varepsilon z\left(\frac{A_{\tau, \lambda, \delta}^{\sigma}(\alpha, \beta) f(z)}{z}\right)^{\prime} \\
& =g(z)+\varepsilon z g^{\prime}(z) \prec h(z) .
\end{aligned}
$$

An application of Lemma 1.1 with $\mu=\frac{1}{\varepsilon}$, yields

$$
g(z) \prec h(z) .
$$

Evidently, $0 \leq \frac{\eta}{\varepsilon}<1$ and that $h$ is convex univalent in $U$, it follows from (2.1), (2.2) and (2.3) that

$$
\begin{aligned}
& \frac{1}{z}\left[\left(1-\frac{\eta(\tau+\lambda)}{(\lambda-\alpha) \beta+n \delta}\right) A_{\tau, \lambda, \delta}^{\sigma}(\alpha, \beta) f(z)+\frac{\eta(\tau+\lambda)}{(\lambda-\alpha) \beta+n \delta} A_{\tau, \lambda, \delta}^{\sigma+1}(\alpha, \beta) f(z)\right] \\
= & \frac{\eta}{\varepsilon z}\left[\left(1-\frac{\varepsilon(\tau+\lambda)}{(\lambda-\alpha) \beta+n \delta}\right) A_{\tau, \lambda, \delta}^{\sigma}(\alpha, \beta) f(z)+\frac{\varepsilon(\tau+\lambda)}{(\lambda-\alpha) \beta+n \delta} A_{\tau, \lambda, \delta}^{\sigma+1}(\alpha, \beta) f(z)\right] \\
& +\left(1-\frac{\eta}{\varepsilon}\right) g(z) \prec h(z) .
\end{aligned}
$$

Hence, $f \in \mathcal{M}(\eta, \sigma, \tau, \lambda, \delta, \alpha, \beta, m ; h)$ and the proof of Theorem 2.1 is completed.

Theorem 2.2. Let $\operatorname{Re}\left\{\frac{\tau+\lambda}{(\lambda-\alpha) \beta+n \delta}\right\} \geq 0$ and $\frac{\tau+\lambda}{(\lambda-\alpha) \beta+n \delta} \neq 0$. Then

$$
\mathcal{M}(\eta, \sigma+1, \tau, \lambda, \delta, \alpha, \beta, m ; h) \subset \mathcal{M}(\eta, \sigma, \tau, \lambda, \delta, \alpha, \beta, m ; h) .
$$

Proof. Let $f \in \mathcal{M}(\eta, \sigma+1, \tau, \lambda, \delta, \alpha, \beta, m ; h)$ and suppose that

$$
g(z)=\frac{1}{z}\left[\left(1-\frac{\eta(\tau+\lambda)}{(\lambda-\alpha) \beta+n \delta}\right) A_{\tau, \lambda, \delta}^{\sigma}(\alpha, \beta) f(z)+\frac{\eta(\tau+\lambda)}{(\lambda-\alpha) \beta+n \delta} A_{\tau, \lambda, \delta}^{\sigma+1}(\alpha, \beta) f(z)\right] .
$$

By taking the derivatives in the both sides of (2.4) with respect to $z$ and using (1.3), we conclude that

$$
\begin{aligned}
& g(z)+z g^{\prime}(z) \\
= & \frac{1}{z}\left[\left(1-\frac{\eta(\tau+\lambda)}{(\lambda-\alpha) \beta+n \delta}\right)\left(1-\frac{\tau+\lambda}{(\lambda-\alpha) \beta+n \delta}\right) A_{\tau, \lambda, \delta}^{\sigma}(\alpha, \beta) f(z)\right. \\
& +\left(1+\eta\left(1-\frac{2(\tau+\lambda)}{(\lambda-\alpha) \beta+n \delta}\right)\right) \frac{\tau+\lambda}{(\lambda-\alpha) \beta+n \delta} A_{\tau, \lambda, \delta}^{\sigma+1}(\alpha, \beta) f(z) \\
& \left.+\eta\left(\frac{\tau+\lambda}{(\lambda-\alpha) \beta+n \delta}\right)^{2} A_{\tau, \lambda, \delta}^{\sigma+2}(\alpha, \beta) f(z)\right] .
\end{aligned}
$$

In the light of (2.4) and (2.5), we deduce that

$$
\frac{\tau+\lambda}{(\lambda-\alpha) \beta+n \delta} g(z)+z g^{\prime}(z)
$$




$$
\begin{aligned}
= & \frac{1}{z}\left[\left(1-\frac{\eta(\tau+\lambda)}{(\lambda-\alpha) \beta+n \delta}\right)\left(\frac{\tau+\lambda}{(\lambda-\alpha) \beta+n \delta}\right) A_{\tau, \lambda, \delta}^{\sigma+1}(\alpha, \beta) f(z)\right. \\
& \left.+\eta\left(\frac{\tau+\lambda}{(\lambda-\alpha) \beta+n \delta}\right)^{2} A_{\tau, \lambda, \delta}^{\sigma+2}(\alpha, \beta) f(z)\right],
\end{aligned}
$$

that is

$$
\begin{aligned}
g(z)+\frac{(\lambda-\alpha) \beta+n \delta}{\tau+\lambda} z g^{\prime}(z)= & \frac{1}{z}\left[\left(1-\frac{\eta(\tau+\lambda)}{(\lambda-\alpha) \beta+n \delta}\right) A_{\tau, \lambda, \delta}^{\sigma+1}(\alpha, \beta) f(z)\right. \\
& \left.+\frac{\eta(\tau+\lambda)}{(\lambda-\alpha) \beta+n \delta} A_{\tau, \lambda, \delta}^{\sigma+2}(\alpha, \beta) f(z)\right] .
\end{aligned}
$$

Since $f \in \mathcal{M}(\eta, \sigma+1, \tau, \lambda, \delta, \alpha, \beta, m ; h)$, then it follows from (2.6) that

$$
g(z)+\frac{(\lambda-\alpha) \beta+n \delta}{\tau+\lambda} z g^{\prime}(z) \prec h(z),
$$

where

$$
\operatorname{Re}\left\{\frac{\tau+\lambda}{(\lambda-\alpha) \beta+n \delta}\right\} \geq 0, \quad \frac{\tau+\lambda}{(\lambda-\alpha) \beta+n \delta} \neq 0 .
$$

An application of Lemma 1.1, with $\mu=\frac{\tau+\lambda}{(\lambda-\alpha) \beta+n \delta}$, yields $g(z) \prec h(z)$. In view of (2.4), we have

$$
\frac{1}{z}\left[\left(1-\frac{\eta(\tau+\lambda)}{(\lambda-\alpha) \beta+n \delta}\right) A_{\tau, \lambda, \delta}^{\sigma}(\alpha, \beta) f(z)+\frac{\eta(\tau+\lambda)}{(\lambda-\alpha) \beta+n \delta} A_{\tau, \lambda, \delta}^{\sigma+1}(\alpha, \beta) f(z)\right] \prec h(z) .
$$

This shows that $f \in \mathcal{M}(\eta, \sigma, \tau, \lambda, \delta, \alpha, \beta, m ; h)$ and the proof of Theorem 2.2 is completed.

Theorem 2.3. Let $\eta>0, \gamma>0$ and $f \in \mathcal{M}(\eta, \sigma, \tau, \lambda, \delta, \alpha, \beta, m ; \gamma h+1-\gamma)$. If $\gamma \leq \gamma_{0}$, where

$$
\gamma_{0}=\frac{1}{2}\left(1-\frac{1}{\eta} \int_{0}^{1} \frac{u^{\frac{1}{\eta}-1}}{1+u} d u\right)^{-1}
$$

then $f \in \mathcal{M}(0, \sigma, \tau, \lambda, \delta, \alpha, \beta, m ; h)$. The bound $\gamma_{0}$ is the sharp when $h(z)=\frac{1}{1-z}$.

Proof. Assume that

$$
g(z)=\frac{A_{\tau, \lambda, \delta}^{\sigma}(\alpha, \beta) f(z)}{z}
$$

Let $f \in \mathcal{M}(\eta, \sigma, \tau, \lambda, \delta, \alpha, \beta, m ; \gamma h+1-\gamma)$ with $\eta>0$ and $\gamma>0$. Then we obtain

$$
\begin{aligned}
& g(z)+\eta z g^{\prime}(z) \\
= & \frac{1}{z}\left[\left(1-\frac{\eta(\tau+\lambda)}{(\lambda-\alpha) \beta+n \delta}\right) A_{\tau, \lambda, \delta}^{\sigma}(\alpha, \beta) f(z)+\frac{\eta(\tau+\lambda)}{(\lambda-\alpha) \beta+n \delta} A_{\tau, \lambda, \delta}^{\sigma+1}(\alpha, \beta) f(z)\right] \\
\prec & \gamma h(z)+1-\gamma .
\end{aligned}
$$


Making use of Lemma 1.1, we observe that

$$
g(z) \prec \frac{\gamma}{\eta} z^{-\frac{1}{\eta}} \int_{0}^{z} t^{\frac{1}{\eta}-1} h(t) d t+1-\gamma=(h * \phi)(z),
$$

where

$$
\phi(z)=\frac{\gamma}{\eta} z^{-\frac{1}{\eta}} \int_{0}^{z} \frac{t^{\frac{1}{\eta}-1}}{1-t} d t+1-\gamma .
$$

If $0<\gamma \leq \gamma_{0}$, where $\gamma_{0}>1$ is given by (2.7), then we find from (2.10) that

$$
\operatorname{Re}(\phi(z))=\frac{\gamma}{\eta} \int_{0}^{1} u^{\frac{1}{\eta}-1} \operatorname{Re}\left(\frac{1}{1-u z}\right) d u+1-\gamma>\frac{\gamma}{\eta} \int_{0}^{1} \frac{u^{\frac{1}{\eta}-1}}{1+u} d u+1-\gamma \geq \frac{1}{2}
$$

By using (2.8) and (2.9), we have

$$
\frac{A_{\tau, \lambda, \delta}^{\sigma}(\alpha, \beta) f(z)}{z} \prec(h * \phi)(z) .
$$

In the light of (2.11), we note that the function $\phi(z)$ has the Herglotz representation

$$
\phi(z)=\int_{|x|=1} \frac{d \mu(x)}{1-x z} \quad(z \in U),
$$

where $\mu(x)$ is a probability measure defined on the unit circle $|x|=1$ and

$$
\int_{|x|=1} d \mu(x)=1 \text {. }
$$

Since $h$ is convex univalent in $U$, then we deduce from (2.12) and (2.13) that

$$
\frac{A_{\tau, \lambda, \delta}^{\sigma}(\alpha, \beta) f(z)}{z} \prec(h * \phi)(z)=\int_{|x|=1} \phi(x z) d \mu(x) \prec h(z) .
$$

This shows that $f \in \mathcal{M}(0, \sigma, \tau, \lambda, \delta, \alpha, \beta, m ; h)$. For $h(z)=\frac{1}{1-z}$ and $f \in \mathcal{A}_{m}$ defined by

$$
\frac{A_{\tau, \lambda, \delta}^{\sigma}(\alpha, \beta) f(z)}{z}=\frac{\gamma}{\eta} z^{-\frac{1}{\eta}} \int_{0}^{z} \frac{t^{\frac{1}{\eta}-1}}{1-t} d t+1-\gamma
$$

we obtain

$$
\begin{aligned}
& \frac{1}{z}\left[\left(1-\frac{\eta(\tau+\lambda)}{(\lambda-\alpha) \beta+n \delta}\right) A_{\tau, \lambda, \delta}^{\sigma}(\alpha, \beta) f(z)+\frac{\eta(\tau+\lambda)}{(\lambda-\alpha) \beta+n \delta} A_{\tau, \lambda, \delta}^{\sigma+1}(\alpha, \beta) f(z)\right] \\
= & \gamma h(z)+1-\gamma .
\end{aligned}
$$

Thus, $f \in \mathcal{M}(\eta, \sigma, \tau, \lambda, \delta, \alpha, \beta, m ; \gamma h+1-\gamma)$. Also, for $\gamma>\gamma_{0}$, we have

$$
\operatorname{Re}\left\{\frac{A_{\tau, \lambda, \delta}^{\sigma}(\alpha, \beta) f(z)}{z}\right\} \rightarrow \frac{\gamma}{\eta} \int_{0}^{1} \frac{u^{\frac{1}{\eta}-1}}{1+u} d u+1-\gamma<\frac{1}{2} \quad(z \rightarrow-1),
$$

which implies that $f \notin \mathcal{M}(0, \sigma, \tau, \lambda, \delta, \alpha, \beta, m ; h)$. Thus, the bound $\gamma_{0}$ cannot be increased when $h(z)=\frac{1}{1-z}$. This completes the proof of the theorem. 
Theorem 2.4. Let $f \in \mathcal{M}(\eta, \sigma, \tau, \lambda, \delta, \alpha, \beta, m ; h)$ be defined as in (1.1). Then the function I defined by

$$
I(z)=\frac{c+1}{z^{c}} \int_{0}^{z} t^{c-1} f(t) d t \quad(\operatorname{Re}(c)>-1)
$$

is also in the class $\mathcal{M}(\eta, \sigma, \tau, \lambda, \delta, \alpha, \beta, m ; h)$.

Proof. Let $f \in \mathcal{M}(\eta, \sigma, \tau, \lambda, \delta, \alpha, \beta, m ; h)$ be defined as in (1.1). Then, we find that (2.14)

$\frac{1}{z}\left[\left(1-\frac{\eta(\tau+\lambda)}{(\lambda-\alpha) \beta+n \delta}\right) A_{\tau, \lambda, \delta}^{\sigma}(\alpha, \beta) f(z)+\frac{\eta(\tau+\lambda)}{(\lambda-\alpha) \beta+n \delta} A_{\tau, \lambda, \delta}^{\sigma+1}(\alpha, \beta) f(z)\right] \prec h(z)$.

We can easily see that

$$
I(z)=\frac{c+1}{z^{c}} \int_{0}^{z} t^{c-1} f(t) d t=z+\sum_{n=m+1}^{\infty} \frac{c+1}{c+n} a_{n} z^{n} .
$$

We have from (2.15) that $I \in \mathcal{A}_{m}$ and

$$
f(z)=\frac{c I(z)+z I^{\prime}(z)}{c+1} .
$$

Define the function $J$ by

$$
J(z)=\frac{1}{z}\left[\left(1-\frac{\eta(\tau+\lambda)}{(\lambda-\alpha) \beta+n \delta}\right) A_{\tau, \lambda, \delta}^{\sigma}(\alpha, \beta) I(z)+\frac{\eta(\tau+\lambda)}{(\lambda-\alpha) \beta+n \delta} A_{\tau, \lambda, \delta}^{\sigma+1}(\alpha, \beta) I(z)\right] .
$$

Differentiating both sides of (2.17) with respect to $z$ and using (2.14) and (2.16), we obtain

$$
\begin{aligned}
& \frac{1}{z}\left[\left(1-\frac{\eta(\tau+\lambda)}{(\lambda-\alpha) \beta+n \delta}\right) A_{\tau, \lambda, \delta}^{\sigma}(\alpha, \beta) f(z)+\frac{\eta(\tau+\lambda)}{(\lambda-\alpha) \beta+n \delta} A_{\tau, \lambda, \delta}^{\sigma+1}(\alpha, \beta) f(z)\right] \\
=\frac{1}{z} & {\left[\left(1-\frac{\eta(\tau+\lambda)}{(\lambda-\alpha) \beta+n \delta}\right) A_{\tau, \lambda, \delta}^{\sigma}(\alpha, \beta)\left(\frac{c I(z)+z I^{\prime}(z)}{c+1}\right)\right.} \\
& \left.+\frac{\eta(\tau+\lambda)}{(\lambda-\alpha) \beta+n \delta} A_{\tau, \lambda, \delta}^{\sigma+1}(\alpha, \beta)\left(\frac{c I(z)+z I^{\prime}(z)}{c+1}\right)\right] \\
= & \frac{c}{z(c+1)}\left[\left(1-\frac{\eta(\tau+\lambda)}{(\lambda-\alpha) \beta+n \delta}\right) A_{\tau, \lambda, \delta}^{\sigma}(\alpha, \beta) I(z)\right. \\
& \left.+\frac{\eta(\tau+\lambda)}{(\lambda-\alpha) \beta+n \delta} A_{\tau, \lambda, \delta}^{\sigma+1}(\alpha, \beta) I(z)\right] \\
& +\frac{1}{z(c+1)}\left[\left(1-\frac{\eta(\tau+\lambda)}{(\lambda-\alpha) \beta+n \delta}\right) A_{\tau, \lambda, \delta}^{\sigma}(\alpha, \beta)\left(z I^{\prime}(z)\right)\right. \\
& \left.+\frac{\eta(\tau+\lambda)}{(\lambda-\alpha) \beta+n \delta} A_{\tau, \lambda, \delta}^{\sigma+1}(\alpha, \beta)\left(z I^{\prime}(z)\right)\right] \\
= & \frac{c}{c+1} J(z)+\frac{1}{c+1}\left(z J^{\prime}(z)+J(z)\right)=J(z)+\frac{1}{c+1} z J^{\prime}(z) \prec h(z) .
\end{aligned}
$$


An application of Lemma 1.1 with $\mu=c+1$, yields $J(z) \prec h(z)$. By using (2.17), we get

$$
\frac{1}{z}\left[\left(1-\frac{\eta(\tau+\lambda)}{(\lambda-\alpha) \beta+n \delta}\right) A_{\tau, \lambda, \delta}^{\sigma}(\alpha, \beta) I(z)+\frac{\eta(\tau+\lambda)}{(\lambda-\alpha) \beta+n \delta} A_{\tau, \lambda, \delta}^{\sigma+1}(\alpha, \beta) I(z)\right] \prec h(z),
$$

which implies that $I \in \mathcal{M}(\eta, \sigma, \tau, \lambda, \delta, \alpha, \beta, m ; h)$.

Theorem 2.5. Let $f \in \mathcal{M}(\eta, \sigma, \tau, \lambda, \delta, \alpha, \beta, m ; h), g \in \mathcal{A}_{m}$ and

$$
\operatorname{Re}\left\{\frac{g(z)}{z}\right\}>\frac{1}{2}
$$

Then $f * g \in \mathcal{M}(\eta, \sigma, \tau, \lambda, \delta, \alpha, \beta, m ; h)$.

Proof. Let $f \in \mathcal{M}(\eta, \sigma, \tau, \lambda, \delta, \alpha, \beta, m ; h)$ and $g \in \mathcal{A}_{m}$. Then, we have

$$
\begin{aligned}
& \frac{1}{z}\left[\left(1-\frac{\eta(\tau+\lambda)}{(\lambda-\alpha) \beta+n \delta}\right) A_{\tau, \lambda, \delta}^{\sigma}(\alpha, \beta)(f * g)(z)\right. \\
& \left.+\frac{\eta(\tau+\lambda)}{(\lambda-\alpha) \beta+n \delta} A_{\tau, \lambda, \delta}^{\sigma+1}(\alpha, \beta)(f * g)(z)\right] \\
& =\left(1-\frac{\eta(\tau+\lambda)}{(\lambda-\alpha) \beta+n \delta}\right)\left(\frac{g(z)}{z}\right) *\left(\frac{A_{\tau, \lambda, \delta}^{\sigma}(\alpha, \beta) f(z)}{z}\right) \\
& +\frac{\eta(\tau+\lambda)}{(\lambda-\alpha) \beta+n \delta}\left(\frac{g(z)}{z}\right) *\left(\frac{A_{\tau, \lambda, \delta}^{\sigma+1}(\alpha, \beta) f(z)}{z}\right)=\left(\frac{g(z)}{z}\right) * \varphi(z),
\end{aligned}
$$

where

$$
\begin{aligned}
\varphi(z) & =\frac{1}{z}\left[\left(1-\frac{\eta(\tau+\lambda)}{(\lambda-\alpha) \beta+n \delta}\right) A_{\tau, \lambda, \delta}^{\sigma}(\alpha, \beta) f(z)+\frac{\eta(\tau+\lambda)}{(\lambda-\alpha) \beta+n \delta} A_{\tau, \lambda, \delta}^{\sigma+1}(\alpha, \beta) f(z)\right] \\
& \prec h(z) .
\end{aligned}
$$

In view of (2.18), we note that the function $\frac{g(z)}{z}$ has the Herglotz representation

$$
\frac{g(z)}{z}=\int_{|x|=1} \frac{d \mu(x)}{1-x z} \quad(z \in U)
$$

where $\mu(x)$ is a probability measure defined on the unit circle $|x|=1$ and

$$
\int_{|x|=1} d \mu(x)=1
$$

Since $h$ is convex univalent in $U$, then we find from (2.19), (2.20) and (2.21) that

$$
\begin{aligned}
& \frac{1}{z}\left[\left(1-\frac{\eta(\tau+\lambda)}{(\lambda-\alpha) \beta+n \delta}\right) A_{\tau, \lambda, \delta}^{\sigma}(\alpha, \beta)(f * g)(z)+\frac{\eta(\tau+\lambda)}{(\lambda-\alpha) \beta+n \delta}\right. \\
& \left.\quad \times A_{\tau, \lambda, \delta}^{\sigma+1}(\alpha, \beta)(f * g)(z)\right]
\end{aligned}
$$




$$
=\int_{|x|=1} \varphi(x z) d \mu(x) \prec h(z) .
$$

This shows that $f * g \in \mathcal{M}(\eta, \sigma, \tau, \lambda, \delta, \alpha, \beta, m ; h)$.

Theorem 2.6. Let $f \in \mathcal{M}(\eta, \sigma, \tau, \lambda, \delta, \alpha, \beta, m ; h)$ and $g \in \mathcal{A}_{m}$ be prestarlike of order $\alpha,(\alpha<1)$. Then $f * g \in \mathcal{M}(\eta, \sigma, \tau, \lambda, \delta, \alpha, \beta, m ; h)$.

Proof. For $f \in \mathcal{M}(\eta, \sigma, \tau, \lambda, \delta, \alpha, \beta, m ; h)$ and $g \in \mathcal{A}_{m}$, from (2.19) (used in the proof of Theorem 2.5), we can write

$$
\begin{aligned}
& \frac{1}{z}\left[\left(1-\frac{\eta(\tau+\lambda)}{(\lambda-\alpha) \beta+n \delta}\right) A_{\tau, \lambda, \delta}^{\sigma}(\alpha, \beta)(f * g)(z)+\frac{\eta(\tau+\lambda)}{(\lambda-\alpha) \beta+n \delta}\right. \\
& \left.\quad \times A_{\tau, \lambda, \delta}^{\sigma+1}(\alpha, \beta)(f * g)(z)\right] \\
& =\frac{g(z) *(z \varphi(z))}{g(z) * z} \quad(z \in U),
\end{aligned}
$$

where $\varphi(z)$ is defined as in (2.20). Since $h$ is convex univalent in $U, \psi(z) \prec h(z)$, $g(z) \in \operatorname{Re}(\alpha)$ and $z \in S^{*}(\alpha), \alpha<1$, it follows from (2.22) and Lemma 1.2, we obtain the result.

\section{REFERENCES}

[1] F. M. Al-Oboudi, On univalent functions defined by a generalized Sãlãgean operator, Int. J. Math. Math. Sci. 27 (2004), 1429-1436.

[2] A. Amourah and M. Darus, Some properties of a new class of univalent functions involving a new generalized differential operator with negative coefficients, Indian Journal of Science and Technology 9(36) (2016), 1-7.

[3] W. G. Atshan and A. K. Wanas, Differential subordinations of multivalent analytic functions associated with Ruscheweyh derivative, Analele Universitǎtii din Oradea - Fascicola Matematică XX(1) (2013), 27-33.

[4] N. E. Cho and T. H. Kim, Multiplier transformations and strongly close-to-convex functions, Bull. Korean Math. Soc. 40(3) (2003), 399-410.

[5] M. Darus and R. W. Ibrahim, On subclasses for generalized operators of complex order, Far East Journal of Mathematical Sciences 33(3) (2009), 299-308.

[6] P. L. Duren, Univalent Functions, Grundlehren der Mathematischen Wissenschaften, Band 259, Springer Verlag, New York, Berlin, Heidelberg, Tokyo, 1983.

[7] J. L. Liu, On a class of multivalent analytic functions associated with an integral operator, Bulletin of the Institute of Mathematics 5(1) (2010), 95-110.

[8] S. S. Miller and P. T. Mocanu, Differential subordinations and univalent functions, Michigan Math. J. 28 (1981), 157-171.

[9] J. K. Prajapat and R. K. Raina, Some applications of differential subordination for a general class of multivalently analytic functions involving a convolution structure, Math. J. Okayama Univ. 52 (2010), 147-158.

[10] S. Ruscheweyh, Convolutions in Geometric Function Theory, Les Presses de I'Université de Montréal, Montréal, 1982.

[11] G. St. Sălăgean, Subclasses of univalent functions, Lecture Notes in Mathematics 1013, Springer Verlag, Berlin, 1983, 362-372. 
[12] A. K. Wanas, Differential subordination results defined by new class for higher-order derivatives of multivalent analytic functions, International Journal of Advanced Research in Science, Engineering and Technology 4(8) (2017), 4363-4368.

[13] A. K. Wanas and A. H. Majeed, Differential subordinations for higher-order derivatives of multivalent analytic functions associated with Dziok-Srivastava operator, Analele Universitǎtii din Oradea - Fascicola Matematicǎ XXV(1) (2018), 33-42.

${ }^{1}$ Department of Mathematics,

College of Science, University of Al-QAdisiyah, IRAQ

Email address: abbas.kareem.w@qu.edu.iq 
Kragujevac Journal of Mathematics

Volume 46(1) (2022), Pages 125-137.

\title{
PSEUDO-BCK ALGEBRAS DERIVED FROM DIRECTOIDS
}

\author{
AKBAR REZAEI ${ }^{1}$
}

\begin{abstract}
The aim of this paper is to derive pseudo-BCK algebras from directoids and vice versa. We generalize some results proved by Ivan Chajda et al. in the case of BCK-algebras. We assign to an arbitrary pseudo-BCK algebra a semilattice-like structure and observe that this is the point where directoids are different from the semilattice-like structures. Finally, the relation between commutative deductive systems and derive directoids from a bounded pseudo-BCK(pDN) algebras and a characterization of commutative deductive systems of a bounded pseudo-BCK $(\mathrm{pDN})$ algebra in terms of directoids is discussed.
\end{abstract}

\section{INTRODUCTION}

BCK-algebras were introduced by Y. Imai and K. Iséki in 1966 ([15, 19]) as algebras with a binary operation $*$ modeling the set-theoretical difference and with a constant element 0 that is a least element. S. Tanaka defined a special class of BCK-algebras called commutative BCK-algebras in 1975 (see [31]). In BCK-algebras, some lattices, as bounded commutative BCK-algebras, involutive BCK-lattices and bounded implicative BCK-algebras were defined and among the relationship between them were discussed [23]. Some recent researchers led to generalizations of the notion of pseudo structure on some types of algebras. G. Georgescu et al. [10] and independently J. Rachůnek [24], introduced pseudo-MV algebra which is a non-commutative generalization of MV-algebra. After a pseudo-MV algebra, the pseudo-BL algebra [11], the pseudo-BCK algebra [12] and as a generalization of BCI-algebra, the notion of pseudo-BCI algebra is introduced by W. A. Dudek et al. in [9]. A. Walendziak [32] introduced pseudo-BCH algebras as an extension of BCH-algebras. Further, he

Key words and phrases. (Commutative) pseudo-BCK algebra, pseudo-BCK lattice, (commutative) deductive system, directoid, semilattice.

2010 Mathematics Subject Classification. 03G25, 06F35, 03F50.

DOI 10.46793/KgJMat2201.125R

Received: November 30, 2018.

Accepted: September 11, 2019. 
proved that every branchwise commutative pseudo-BCH algebra is a pseudo-BCI algebra [33]. Commutative pseudo-BCK algebras were originally defined by G. Georgescu et al. in [12] under the name of semilattice-ordered pseudo-BCK algebras and some properties of these structures were investigated by J. Kühr in [21,22]. R. A. Borzooei et al. introduced in [1] (see also $[2,26,27]$ ) a pseudo-BE algebra as generalization of BE-algebra, and the commutative pseudo-BE algebra have recently been investigated by L. C. Ciungu. It was proved that the class of commutative pseudo-BE algebras is equivalent to the class of commutative pseudo-BCK algebras. Based on this result, all results holding for commutative pseudo-BCK algebras also hold for commutative pseudo-BE algebras [5]. Then she gave a characterization of commutative pseudo-BCK algebras and defined the commutative deductive systems of pseudo-BCK algebras and proved that a pseudo-BCK algebra $\mathfrak{X}$ is commutative if and only if all the deductive systems of $\mathfrak{X}$ are commutative. Also, she showed that the class of commutative pseudo-BCK algebras is a variety [6] (see also, [14]). A. Rezaei et al. introduced the notion of pseudo-CI algebras as an extension of pseudo-BE algebras and proved that the class of commutative pseudo-CI algebras coincide with the class of commutative pseudo-BCK algebras [28]. G. Georgescu et al. proved that every Wajsberg pseudo-hoop is a basic pseudo-hoop and every simple basic pseudo-hoop is a linearly ordered Wajsberg pseudo-hoop [13]. L. C. Ciungu in [7] showed that every pseudo-hoop is a pseudo-BCK-meet semilattice. The relation between $\mathrm{FL}_{w}$-algebras, bounded pseudo-BCK $(\mathrm{pP})$ algebras, pseudo-MTL algebras, pseudo-BL algebras and pseudo-MV algebras proved in [16]. Also, in [29,30], the interrelationships between dual pseudo-Q/QC algebras and other pseudo algebras are visualized with a diagram and then they introduced the concepts of branchwise commutative pseudo-CI algebras and pointed pseudo-CI algebras and investigated some of properties. A. Iorgulescu for the first time introduced the notation of quasi-pseudo-M algebras as generalizations of pseudo-M algebras and (involutive) quasi-implicative-groups and the (strong involutive) (super) quasi-implicative-hoops, as generalizations of implicative-groups and implicative-hoops, respectively in [18]. I. Chajda et al. showed that one can be assign to an arbitrary BCK-algebra a semilattice-like structure every section of which possesses a certain antitone mappings [3], it arises a natural question of generalization of these concepts also for pseudo-BCK algebras. Since lattice theory has many applications in computer science and has an important and vital role in investigating the structure of a logical system, this motivated our investigations on directoids and pseudo-BCK-algebras to characterized several of its important properties. The main result of this paper establishes a bijective correspondence between pseudo-BCK algebras and some algebraic structures defined by two directoids. A characterization of commutative deductive systems of a bounded pseudo-BCK $(\mathrm{pDN})$ algebra in terms of directoids is discussed and various results obtained mentioned in this paper can be transferred to the pseudo-BCK algebras. The core of the paper is based on by presenting a survey of some results of logic in the non-commutative case (see [3] for the commutative case) and extension of [25] (see also [4]). 


\section{Preliminaries}

In this section we recall some basic notions and results regarding (commutative) pseudo-BCK algebras.

Definition $2.1([9,17])$. An algebra $\mathfrak{X}=(X ; \rightarrow, \rightsquigarrow, 1)$ of type $(2,2,0)$ is called a pseudo-BCI algebra if it satisfies the following axioms for all $x, y, z \in X$ :

$\left(\mathrm{psBCI}_{1}\right)(x \rightarrow y) \rightsquigarrow((y \rightarrow z) \rightsquigarrow(x \rightarrow z))=1$ and $(x \rightsquigarrow y) \rightarrow((y \rightsquigarrow z) \rightarrow(x \rightsquigarrow z))=1$;

$\left(\mathrm{psBCI}_{2}\right) x \rightarrow((x \rightarrow y) \rightsquigarrow y)=1$ and $x \rightsquigarrow((x \rightsquigarrow y) \rightarrow y)=1$;

$\left(\mathrm{psBCI}_{3}\right) x \rightarrow x=x \rightsquigarrow x=1$;

$\left(\mathrm{psBCI}_{4}\right) x \rightarrow y=y \rightsquigarrow x=1 \Rightarrow x=y$;

$\left(\operatorname{psBCI}_{5}\right) x \preceq y$ if and only if $x \rightarrow y=1$ if and only if $x \rightsquigarrow y=1$.

A pseudo-BCK algebra [20] is a pseudo-BCI algebra $\mathfrak{X}=(X ; \rightarrow, \rightsquigarrow, 1)$ satisfying the condition (psBCK), for all $x \in X$ :

$(\mathrm{psBCK}) x \rightarrow 1=1$.

I. Chajda et al. proved that for every pseudo-BCI algebra $x \rightarrow y=1$ if and only if $x \rightsquigarrow y=1$ (see [4, Lemma 2.1]).

Remark 2.1. If $\mathfrak{X}=(X ; \rightarrow, \rightsquigarrow, 1)$ is a pseudo-BCI algebra satisfying $x \rightarrow y=x \rightsquigarrow y$, for all $x, y \in X$, then $\mathfrak{X}=(X ; \rightarrow, 1)$ is a BCI-algebra. Hence, every BCI-algebra is a pseudo-BCI algebra in a natural way.

Remark 2.2. By definition ( $\left.\mathrm{psBCI}_{1}\right)-\left(\mathrm{psBCI}_{5}\right)$, pseudo-BCK algebras are contained in the class of pseudo-BCI algebras. A pseudo-BCI algebra which is not a pseudo-BCK algebra will be called proper.

From now on, $\mathfrak{X}$ is a pseudo-BCK algebra, unless it is stated.

Proposition 2.1 ([12,17]). In any pseudo-BCK algebra $\mathfrak{X}$ the following conditions hold for all $x, y, z \in X$ :

(1) $x \preceq y$ implies $z \rightarrow x \preceq z \rightarrow y$ and $z \rightsquigarrow x \preceq z \rightsquigarrow y$;

(2) $x \preceq y$ implies $y \rightarrow z \preceq x \rightarrow z$ and $y \rightsquigarrow z \preceq x \rightsquigarrow z$;

(3) $x \rightarrow y \preceq(z \rightarrow x) \rightsquigarrow(z \rightarrow y)$ and $x \rightsquigarrow y \preceq(z \rightsquigarrow x) \rightarrow(z \rightsquigarrow y)$;

(4) $x \rightarrow(y \rightsquigarrow z)=y \rightsquigarrow(x \rightarrow z)$ and $x \rightsquigarrow(y \rightarrow z)=y \rightarrow(x \rightsquigarrow z)$;

(5) $x \preceq y \rightarrow x$ and $x \preceq y \rightsquigarrow x$;

(6) $((x \rightarrow y) \rightsquigarrow y) \rightarrow y=x \rightarrow y$ and $((x \rightsquigarrow y) \rightarrow y) \rightsquigarrow y=x \rightsquigarrow y$.

Theorem 2.1 ([6]). Let $\mathfrak{X}$ be a pseudo-BCK algebra. The following statements are equivalent for all $x, y \in X$ :

(1) $\mathfrak{X}$ is commutative;

(2) $x \rightarrow y=((y \rightarrow x) \rightsquigarrow x) \rightarrow y$ and $x \rightsquigarrow y=((y \rightsquigarrow x) \rightarrow x) \rightsquigarrow y$;

(3) $(x \rightarrow y) \rightsquigarrow y=(((x \rightarrow y) \rightsquigarrow y) \rightarrow x) \rightsquigarrow x$ and $(x \rightsquigarrow y) \rightarrow y=(((x \rightsquigarrow y) \rightarrow y) \rightsquigarrow x) \rightarrow x ;$

(4) $x \preceq y$ implies $y=(y \rightarrow x) \rightsquigarrow x=(y \rightsquigarrow x) \rightarrow x$. 
Definition 2.2 ([16]). If there is an element 0 of a pseudo-BCK algebra $\mathfrak{X}$, such that $0 \preceq x$ (i.e., $0 \rightarrow x=0 \rightsquigarrow x=1$ ), for all $x \in X$, then 0 is called the zero of $\mathfrak{X}$. A pseudo-BCK algebra with zero is called bounded pseudo-BCK algebra and it is denoted by $\mathfrak{X}=(X ; \rightarrow, \rightsquigarrow, 0,1)$.

Definition 2.3 ([16]). A pseudo-BCK $(p P)$ algebra is a pseudo-BCK algebra $\mathfrak{X}$ satisfying $(\mathrm{pP})$ condition:

$(\mathrm{pP})$ There exists $x \odot y=\min \{z: x \preceq y \rightarrow z\}=\min \{z: y \preceq x \rightsquigarrow z\}$ for all $x, y \in X$.

Definition $2.4([16,20])$.

(1) A pseudo-BCK lattice is a pseudo-BCK algebra $\mathfrak{X}$ such that $(X ; \preceq)$ is a lattice.

(2) A pseudo-BCK join-semilattice is a pseudo-BCK algebra $\mathfrak{X}$ such that $(X ; \vee)$ is a join-semilattice, and $x \rightarrow y=1$ if and only if $x \vee y=y$.

(3) A pseudo-BCK meet-semilattice is a pseudo-BCK algebra $\mathfrak{X}$ such that $(X ; \wedge)$ is a meet-semilattice, and $x \rightarrow y=1$ if and only if $x \wedge y=x$.

Definition $2.5([16])$. A pseudo-BCK algebra $(p D N)$ is a bounded pseudo-BCK algebra $\mathfrak{X}=(X ; \preceq, \rightarrow, \rightsquigarrow, 0,1)$ satisfying the condition:

$(\mathrm{pDN})\left(x^{\rightarrow}\right)^{\rightsquigarrow}=\left(x^{\rightsquigarrow}\right) \rightarrow=x$, where $x^{\rightarrow}=x \rightarrow 0$ and $x^{\rightsquigarrow}=x \rightsquigarrow 0$ for all $x \in X$.

Definition 2.6 ([12]). A pseudo-BCK algebra $\mathfrak{X}$ is called commutative if for all $x, y, z \in X$, it satisfies the following identities:

$\left(\mathrm{C}_{1}\right)(x \rightarrow y) \rightsquigarrow y=(y \rightarrow x) \rightsquigarrow x ;$

$\left(\mathrm{C}_{2}\right)(x \rightsquigarrow y) \rightarrow y=(y \rightsquigarrow x) \rightarrow x$.

Proposition 2.2 ([6]). Any commutative pseudo-BCK algebra is a join-semilattice with respect to $\preceq$.

Theorem $2.2([8])$. Let $\mathfrak{X}$ be a pseudo-BCK(pDN) algebra. The following statements are equivalent:

(1) $(X ; \preceq)$ is a meet-semilattice;

(2) $(X ; \preceq)$ is a join-semilattice;

(3) $(X ; \preceq)$ is a lattice.

Definition $2.7([6])$. A subset $D$ of a pseudo-BCK algebra $\mathfrak{X}$ is called a deductive system of $\mathfrak{X}$ if it satisfies the following conditions:

$\left(\mathrm{DS}_{1}\right) 1 \in D$;

$\left(\mathrm{DS}_{2}\right) x \in D$ and $x \rightarrow y \in D$ imply $y \in D$.

A subset $D$ of $\mathfrak{X}$ is a deductive system if and only if it satisfies $\left(\mathrm{DS}_{1}\right)$ and the condition:

$\left(\mathrm{DS}_{3}\right) x \in D$ and $x \rightsquigarrow y \in D$ imply $y \in D$.

We will denote by $\mathfrak{D} S(X)$ the set of all deductive systems of $\mathfrak{X}$.

Definition $2.8([6])$. A deductive system $D$ of a pseudo-BCK algebra $\mathfrak{X}$ is called commutative if it satisfies the following conditions for all $x, y \in X$ : 
$\left(\mathrm{CDS}_{1}\right) y \rightarrow x \in D$ implies $((x \rightarrow y) \rightsquigarrow y) \rightarrow x \in D$;

$\left(\mathrm{CDS}_{2}\right) y \rightsquigarrow x \in D$ implies $((x \rightsquigarrow y) \rightarrow y) \rightsquigarrow x \in D$.

We will denote by $\mathfrak{D} S_{c}(X)$ the set of all commutative deductive systems of a pseudo-BCK algebra $\mathfrak{X}$.

Definition $2.9([3])$. A directoid is a groupoid $\mathfrak{G}=(G ; \vee)$ satisfying the following identities for all $x, y, z \in G$ :

$\left(\mathrm{D}_{1}\right) x \vee x=x$;

$\left(\mathrm{D}_{2}\right)(x \vee y) \vee x=x \vee y$;

$\left(\mathrm{D}_{3}\right) y \vee(x \vee y)=x \vee y$;

$\left(\mathrm{D}_{4}\right) x \vee((x \vee y) \vee z)=(x \vee y) \vee z$.

The relation $\leq$ given by $x \leq y$ if and only if $x \vee y=y$ is a partial order. The binary operation $\vee$ assigns to a pair $\{x, y\}$ is a common upper bound of them.

\section{Pseudo-BCK Algebras Derived from Directoids}

Following the idea used by I. Chajda and J. Kühr [3] for BCK-algebras in what follows we give a generalization of this results for pseudo-BCK algebras. In this section, we assign a semilattice-like structure the sections of which have certain antitone mappings, and also conversely. We have the following results.

Let $\mathfrak{X}$ be a pseudo-BCK algebra. Define binary operations $\vee_{1}$ and $\vee_{2}$ by:

(A) $x \vee_{1} y:=(x \rightarrow y) \rightsquigarrow y$ and $x \vee_{2} y:=(x \rightsquigarrow y) \rightarrow y$ for all $x, y \in X$.

The following examples shows that these operations $\vee_{1}$ and $\vee_{2}$ need not coincide in general.

Example 3.1 ([8]). Consider the set $X=\{0, a, b, c, 1\}$, where $0<a, b<c<1, a, b$ inomparable and the operations $\rightarrow$ and $\rightsquigarrow$ given by the following tables:

\begin{tabular}{c|cccccc|ccccc}
$\rightarrow$ & 0 & $a$ & $b$ & $c$ & 1 & $\rightsquigarrow$ & 0 & $a$ & $b$ & $c$ & 1 \\
\hline 0 & 1 & 1 & 1 & 1 & 1 & 0 & 1 & 1 & 1 & 1 & 1 \\
$a$ & 0 & 1 & $b$ & 1 & 1 & $a$ & $b$ & 1 & $b$ & 1 & 1 \\
$b$ & $a$ & $a$ & 1 & 1 & 1 & $b$ & 0 & $a$ & 1 & 1 & 1 \\
$c$ & 0 & $a$ & $b$ & 1 & 1 & $c$ & 0 & $a$ & $b$ & 1 & 1 \\
1 & 0 & $a$ & $b$ & $c$ & 1 & 1 & 0 & $a$ & $b$ & $c$ & 1
\end{tabular}.

Then $\mathfrak{X}=(X ; \rightarrow, \rightsquigarrow, 0,1)$ is a bounded pseudo-BCK algebra, but

$$
a \vee_{1} 0=(a \rightarrow 0) \rightsquigarrow 0=0 \rightsquigarrow 0=1 \neq a \vee_{2} 0=(a \rightsquigarrow 0) \rightarrow 0=b \rightarrow 0=a
$$

Theorem 3.1. Let $\mathfrak{X}$ be a pseudo-BCK algebra. For every $a \in X$, define unary operations $\rightarrow a$ and ${ }^{\rightsquigarrow a}$ by $x^{\rightarrow a}=x \rightarrow a$ and $x^{\rightsquigarrow a}=x \rightsquigarrow a$. Then the algebraic structure $\mathfrak{S}(\mathfrak{X})=\left(X ; \vee_{1}, \vee_{2},\left({ }^{\rightarrow a}\right)_{a \in X},\left({ }^{\rightsquigarrow a}\right)_{a \in X}, 1\right)$ satisfies the following quasi-identities:

(1) $x \vee_{1} 1=1$ and $x \vee_{2} 1=1$;

(2) $x^{\rightarrow x}=1$ and $x^{\rightsquigarrow x}=1$;

(3) $1^{\rightarrow x}=x$ and $1^{\rightsquigarrow x}=x$;

(4) $x \vee_{1} x=x$ and $x \vee_{2} x=x$; 
(5) $\left(x \vee_{1} y\right)^{\rightarrow y}=x \rightarrow y$ and $\left(x \vee_{2} y\right)^{\rightsquigarrow y}=x \rightsquigarrow y$;

(6) $x \preceq y$ if and only if $x \vee_{1} y=y$ and $x \vee_{2} y=y$;

(7) $x \vee_{1} y=y$ and $y \vee_{1} x=x$ imply $x=y$ and $x \vee_{2} y=y$ and $y \vee_{2} x=x$ imply $x=y$;

(8) $x \vee_{1} y=\left(x \vee_{1} y\right) \vee_{1} y=x \vee_{1}\left(x \vee_{1} y\right)=y \vee_{1}\left(x \vee_{1} y\right)$ and $x \vee_{2} y=\left(x \vee_{2} y\right) \vee_{2} y=x \vee_{2}\left(x \vee_{2} y\right)=y \vee_{2}\left(x \vee_{2} y\right)$

(9) $\left(x \vee_{1} z\right) \vee_{1}\left(\left(x \vee_{1} y\right) \vee_{1} z\right)=\left(x \vee_{1} y\right) \vee_{1} z$ and $\left(x \vee_{2} z\right) \vee_{2}\left(\left(x \vee_{2} y\right) \vee_{2} z\right)=\left(x \vee_{2} y\right) \vee_{2} z$;

(10) $x \vee_{1} y=\left(x \vee_{1} y\right)^{\rightarrow y \rightsquigarrow y}=\left(\left(x \vee_{1} y\right)^{\rightarrow y} \vee_{2} y\right)^{\rightsquigarrow y}$ and $x \vee_{2} y=\left(x \vee_{2} y\right)^{\rightsquigarrow y \rightarrow y}=\left(\left(x \vee_{2} y\right)^{\rightsquigarrow y} \vee_{1} y\right)^{\rightarrow y}$

(11) $\left(x \vee_{1}\left(y \vee_{2} z\right)^{\rightsquigarrow z}\right)^{\rightarrow\left(y \vee_{2} z\right)^{\rightsquigarrow z}}=\left(y \vee_{2}\left(x \vee_{1} z\right)^{\rightarrow z}\right)^{\rightsquigarrow\left(x \vee_{1} z\right)^{\rightarrow z}}$;

(12) $\left(x \vee_{1} y\right)^{\rightarrow y} \vee_{2}\left(\left(x \vee_{1} z\right) \vee_{1}\left(y \vee_{1} z\right)\right) \rightarrow\left(y \vee_{1} z\right)=\left(\left(x \vee_{1} z\right) \vee_{1}\left(y \vee_{1} z\right)\right) \rightarrow\left(y \vee_{1} z\right)$ and $\left(x \vee_{2} y\right)^{\rightsquigarrow y} \vee_{1}\left(\left(x \vee_{2} z\right) \vee_{2}\left(y \vee_{2} z\right)\right)^{\rightsquigarrow\left(y \vee_{2} z\right)}=\left(\left(x \vee_{2} z\right) \vee_{2}\left(y \vee_{2} z\right)\right)^{\rightsquigarrow\left(y \vee_{2} z\right)}$;

(13) $\left(\left(x \vee_{2} z\right)^{\rightsquigarrow z} \vee_{1}\left(y \vee_{1} z\right)\right) \rightarrow\left(y \vee_{1} z\right)=\left(\left(y \vee_{1} z\right)^{\rightarrow z} \vee_{2}\left(x \vee_{2} z\right)\right)^{\rightsquigarrow\left(x \vee_{2} z\right)}$ and $\left(\left(x \vee_{1} z\right)^{\rightarrow z} \vee_{2}\left(y \vee_{2} z\right)\right)^{\rightsquigarrow\left(y \vee_{2} z\right)}=\left(\left(y \vee_{2} z\right)^{\rightsquigarrow z} \vee_{1}\left(x \vee_{1} z\right)\right) \rightarrow\left(x \vee_{1} z\right)$;

(14) $\left(\left(x \vee_{1} y\right) \vee_{1} x\right)^{\rightarrow x}=\left(x \vee_{1} y\right)^{\rightarrow x}$ and $\left(\left(x \vee_{2} y\right) \vee_{2} x\right)^{\rightsquigarrow x}=\left(x \vee_{2} y\right)^{\rightsquigarrow x}$.

Proof. The proof of (1)-(6) is straightforward by the definition and properties of pseudo-BCK algebras. (6) Assume that $x \preceq y$. Then $x \vee_{1} y=(x \rightarrow y) \rightsquigarrow y=1 \rightsquigarrow$ $y=y$. Also,

$$
x \vee_{2} y=(x \rightsquigarrow y) \rightarrow y=1 \rightarrow y=y .
$$

Conversely, suppose that $x \vee_{1} y=y$ and $x \vee_{2} y=y$. Since

$$
x \rightarrow y=x \rightarrow((x \rightarrow y) \rightsquigarrow y)=(x \rightarrow y) \rightsquigarrow(x \rightarrow y)=1
$$

and

$$
x \rightsquigarrow y=x \rightsquigarrow((x \rightsquigarrow y) \rightarrow y)=(x \rightsquigarrow y) \rightarrow(x \rightsquigarrow y)=1,
$$

then $x \preceq y$.

(7) Suppose that $(x \rightarrow y) \rightsquigarrow y=y$ and $(y \rightarrow x) \rightsquigarrow x=x$. Then by $\left(\mathrm{psBCI}_{3}\right)$ we have

$$
x \rightarrow y=x \rightarrow((x \rightarrow y) \rightsquigarrow y)=(x \rightarrow y) \rightsquigarrow(x \rightarrow y)=1
$$

and

$$
y \rightsquigarrow x=y \rightsquigarrow((y \rightsquigarrow x) \rightarrow x)=(y \rightsquigarrow x) \rightarrow(y \rightsquigarrow x)=1 .
$$

Now, using $\left(\mathrm{psBCI}_{5}\right) x=y$. By a similar argument the second part is valid.

(8) By Proposition 2.1 (6), we have

$$
\begin{aligned}
\left(x \vee_{1} y\right) \vee_{1} y & =\left(\left(x \vee_{1} y\right) \rightarrow y\right) \rightsquigarrow y \\
& =(((x \rightarrow y) \rightsquigarrow y) \rightarrow y) \rightsquigarrow y \\
& =(x \rightarrow y) \rightsquigarrow y \\
& =x \vee_{1} y .
\end{aligned}
$$

Similarly, we see that $x \vee_{1}\left(x \vee_{1} y\right)=x \vee_{1} y$ and $y \vee_{1}\left(x \vee_{1} y\right)=x \vee_{1} y$. 
(9) According to $\left(\mathrm{psBCI}_{2}\right), x \preceq x \vee_{1} y$. By Proposition 2.1 (1) and (2), we have $x \vee_{1} z \preceq\left(x \vee_{1} y\right) \vee_{1} z$. Now, using (6) it follows that $\left(x \vee_{1} z\right) \vee_{1}\left(\left(x \vee_{1} y\right) \vee_{1} z\right)=\left(x \vee_{1} y\right) \vee_{1} z$. By a similar argument we can verify $x \vee_{2} y=\left(x \vee_{2} y\right) \vee_{2} y=x \vee_{2}\left(x \vee_{2} y\right)=y \vee_{2}\left(x \vee_{2} y\right)$.

(10) $\left(x \vee_{1} y\right)^{\rightarrow y \rightsquigarrow y}=\left(\left(x \vee_{1} y\right) \rightarrow y\right) \rightsquigarrow y=x \vee_{1} y$ and $\left(x \vee_{2} y\right)^{\rightsquigarrow y \rightarrow y}=\left(\left(x \vee_{2} y\right) \rightsquigarrow\right.$ $y) \rightarrow y=x \vee_{2} y$. By Proposition 2.1 (6), we have

$$
\begin{aligned}
\left(\left(x \vee_{1} y\right)^{\rightarrow y} \vee_{2} y\right)^{\rightsquigarrow y} & =\left(\left(\left(x \vee_{1} y\right) \rightarrow y\right) \vee_{2} y\right)^{\rightsquigarrow y} \\
& =\left((((x \rightarrow y) \rightsquigarrow y) \rightarrow y) \vee_{2} y\right)^{\rightsquigarrow y} \\
& =\left((x \rightarrow y) \vee_{2} y\right)^{\rightsquigarrow y} \\
& =(((x \rightarrow y) \rightsquigarrow y) \rightarrow y)^{\rightsquigarrow y} \\
& =(x \rightarrow y)^{\rightsquigarrow y} \\
& =(x \rightarrow y) \rightsquigarrow y \\
& =x \vee_{1} y .
\end{aligned}
$$

Also, the proof of the second part is similar.

(11) From (5) and Proposition 2.1 (4), we conclude

$$
\begin{aligned}
\left(x \vee_{1}\left(y \vee_{2} z\right)^{\rightsquigarrow z}\right) \rightarrow\left(y \vee_{2} z\right)^{\rightsquigarrow z} & =\left(x \vee_{1}(y \rightsquigarrow z)\right)^{\rightarrow(y \rightsquigarrow z)} \\
& =x \rightarrow(y \rightsquigarrow z)=y \rightsquigarrow(x \rightarrow z) \\
& =\left(y \vee_{2}(x \rightarrow z)\right)^{\rightsquigarrow(x \rightarrow z)} \\
& =\left(y \vee_{2}\left(x \vee_{1} z\right)^{\rightarrow z}\right)^{\rightsquigarrow\left(x \vee_{1} z\right)^{\rightarrow z}} .
\end{aligned}
$$

(12) Using (5), we have

$$
\begin{aligned}
\left(\left(x \vee_{1} z\right) \vee_{1}\left(y \vee_{1} z\right)\right) \rightarrow\left(y \vee_{1} z\right) & =\left(x \vee_{1} z\right) \rightarrow\left(y \vee_{1} z\right) \\
& =((x \rightarrow z) \rightsquigarrow z) \rightarrow((y \rightarrow z) \rightsquigarrow z) \\
& =(y \rightarrow z) \rightsquigarrow(((x \rightarrow z) \rightsquigarrow z) \rightarrow z) \\
& =(y \rightarrow z) \rightsquigarrow(x \rightarrow z) .
\end{aligned}
$$

We have $(x \rightarrow y) \vee_{2}((y \rightarrow z) \rightsquigarrow(x \rightarrow z))=1 \rightarrow((y \rightarrow z) \rightsquigarrow(x \rightarrow z))$. From this and $\left(\mathrm{psBCI}_{1}\right)$ we conclude

$$
\begin{aligned}
\left(x \vee_{1} y\right)^{\rightarrow y} \vee_{2}\left(\left(x \vee_{1} z\right) \vee_{1}\left(y \vee_{1} z\right)\right)^{\rightarrow\left(y \vee_{1} z\right)} & =\left(x \vee_{1} y\right)^{\rightarrow y} \vee_{2}((y \rightarrow z) \rightsquigarrow(x \rightarrow z)) \\
& =(x \rightarrow y) \vee_{2}((y \rightarrow z) \rightsquigarrow(x \rightarrow z)) \\
& =1 \rightarrow((y \rightarrow z) \rightsquigarrow(x \rightarrow z)) \\
& =(y \rightarrow z) \rightsquigarrow(x \rightarrow z) \\
& =\left(\left(x \vee_{1} z\right) \vee_{1}\left(y \vee_{1} z\right)\right) \rightarrow\left(y \vee_{1} z\right)
\end{aligned}
$$


(13) Applying (5), we have

$$
\begin{aligned}
\left(\left(x \vee_{2} z\right)^{\rightsquigarrow z} \vee_{1}\left(y \vee_{1} z\right)\right)^{\rightarrow\left(y \vee_{1} z\right)} & =(x \rightsquigarrow z) \rightarrow\left(y \vee_{1} z\right) \\
& =(x \rightsquigarrow z) \rightarrow((y \rightarrow z) \rightsquigarrow z) \\
& =(y \rightarrow z) \rightsquigarrow((x \rightsquigarrow z) \rightarrow z) \\
& =(y \rightarrow z) \rightsquigarrow\left(x \vee_{2} z\right) \\
& =\left(\left(y \vee_{1} z\right)^{\rightarrow z} \vee_{2}\left(x \vee_{2} z\right)\right)^{\rightsquigarrow\left(x \vee_{2} z\right)} .
\end{aligned}
$$

By a similar argument we have

$$
\left(\left(x \vee_{1} z\right)^{\rightarrow z} \vee_{2}\left(y \vee_{2} z\right)\right)^{\rightsquigarrow\left(y \vee_{2} z\right)}=\left(\left(y \vee_{2} z\right)^{\rightsquigarrow z} \vee_{1}\left(x \vee_{1} z\right)\right)^{\rightarrow\left(x \vee_{1} z\right)}
$$

(14) Using Proposition 2.1 (6), we get

$$
\begin{aligned}
\left(\left(x \vee_{1} y\right) \vee_{1} x\right)^{\rightarrow x} & =\left(((x \rightarrow y) \rightsquigarrow y) \vee_{1} x\right)^{\rightarrow x} \\
& =((((x \rightarrow y) \rightsquigarrow y) \rightarrow x) \rightsquigarrow x)^{\rightarrow x} \\
& =((x \rightarrow y) \rightsquigarrow y)^{\rightarrow x} \\
& =((x \rightarrow y) \rightsquigarrow y) \rightarrow x \\
& =\left(x \vee_{1} y\right) \rightarrow x \\
& =\left(x \vee_{1} y\right)^{\rightarrow x}
\end{aligned}
$$

Similarly, $\left(\left(x \vee_{2} y\right) \vee_{2} x\right)^{\rightsquigarrow x}=\left(x \vee_{2} y\right)^{\rightsquigarrow x}$.

Lemma 3.1. Let $\mathfrak{X}=\left(X ; \vee_{1}, \vee_{2}\right)$ be an algebra of type $(2,2)$ satisfying the quasiidentities (4), (7), (8) and (9) of Theorem 3.1. Then the binary relation $\preceq$ defined by

(B) $x \preceq y$ if and only if $x \vee_{1} y=y$ and $x \vee_{2} y=y$ is a partial order on $X$.

Proof. By (4) and (7), $\preceq$ is reflexive and antisymmetric. For transitivity, assume that $x \preceq y$ and $y \preceq z$. Using (8) and (9), we get

$$
\begin{aligned}
x \vee_{1} z & =\left(x \vee_{1} z\right) \vee_{1} z \\
& =\left(x \vee_{1} z\right) \vee_{1}\left(y \vee_{1} z\right) \\
& =\left(x \vee_{1} z\right) \vee_{1}\left(\left(x \vee_{1} y\right) \vee_{1} z\right) \\
& =\left(x \vee_{1} y\right) \vee_{1} z \\
& =y \vee_{1} z=z
\end{aligned}
$$

and if $x \vee_{2} y=y$ and $y \vee_{2} z=z$, then we have

$$
\begin{aligned}
x \vee_{2} z & =\left(x \vee_{2} z\right) \vee_{2} z \\
& =\left(x \vee_{2} z\right) \vee_{2}\left(y \vee_{2} z\right) \\
& =\left(x \vee_{2} z\right) \vee_{2}\left(\left(x \vee_{2} y\right) \vee_{2} z\right) \\
& =\left(x \vee_{2} y\right) \vee_{2} z \\
& =y \vee_{2} z=z .
\end{aligned}
$$


Thus, $\preceq$ is a partial order on $X$.

The following example shows that for every pseudo-BCK algebra $\mathfrak{X},\left(X ; \vee_{1}\right)$ and $\left(X ; \vee_{2}\right)$ are not directoids in general.

Example 3.2. Let $\mathfrak{X}$ be the algebra given in Example 3.1. Then $\left(X ; \vee_{1}\right)$ and $\left(X ; \vee_{2}\right)$ are not directoids, since

$c \vee_{1} 0=(c \rightarrow 0) \rightsquigarrow 0=0 \rightsquigarrow 0=1 \neq\left(c \vee_{1} 0\right) \vee_{1} c=0 \vee_{1} c=(0 \rightarrow c) \rightsquigarrow c=1 \rightsquigarrow c=c$ and

$c \vee_{2} 0=(c \rightsquigarrow 0) \rightarrow 0=0 \rightarrow 0=1 \neq\left(c \vee_{2} 0\right) \vee_{2} c=0 \vee_{2} c=(0 \rightsquigarrow c) \rightarrow c=1 \rightarrow c=c$.

Theorem 3.2. Let $\mathfrak{X}$ be a pseudo-BCK algebra, $\vee_{1}$ and $\vee_{2}$ be the binary operations defined by $(\mathrm{A})$. Then the following conditions are equivalent:

(1) $\left(X ; \vee_{1}\right)$ and $\left(X ; \vee_{2}\right)$ are directoids;

(2) $\mathfrak{X}$ is a commutative pseudo-BCK algebra;

(3) $(X$; $)$ is a join-semilattice, where $\preceq$ is defined by (B).

Proof. $(1) \Rightarrow(2)$ Assume that $\left(X ; \vee_{1}\right)$ is a directoid. Then $x \preceq y$ implies $y \vee_{1} x=y$ and so $\mathfrak{X}$ satisfies the quasi-identity

$$
x \preceq y \Rightarrow y=(y \rightarrow x) \rightsquigarrow x .
$$

Similary, $x \preceq y$ implies $y=(y \rightsquigarrow x) \rightarrow x$. Therefore, $\mathfrak{X}$ is a commutative pseudo-BCK algebra by Theorem 2.1 .

$(2) \Rightarrow(3)$ It follows from Proposition 2.2.

$(3) \Rightarrow(1)$ It is obvious that every join-semilattice is a directoid.

Corollary 3.1. Let $\mathfrak{X}$ be a pseudo-BCK $(p D N), \vee_{1}$ and $\vee_{2}$ be the binary operations defined by $(\mathrm{A})$. Then the following conditions are equivalent:

(1) $\left(X ; \vee_{1}\right)$ and $\left(X ; \vee_{2}\right)$ are directoids;

(2) $\mathfrak{X}$ is a commutative pseudo-BCK algebra;

(3) $(X ; \preceq)$ is a join-semilattice;

(4) $(X ; \preceq)$ is a meet-semilattice;

(5) $(X ; \preceq)$ is a lattice.

Proof. It follows from Theorems 3.2 and 2.2.

Corollary 3.2. Let $\mathfrak{X}$ be a pseudo- $B C K(p D N), \vee_{1}$ and $\vee_{2}$ be the binary operations defined by $(\mathrm{A})$. Then the following conditions are equivalent:

(1) $\left(X ; \vee_{1}\right)$ and $\left(X ; \vee_{2}\right)$ are directoids;

(2) $\mathfrak{X}$ is a commutative pseudo-BCK algebra;

(3) $\{1\} \in \mathfrak{D} S_{c}(X)$;

(4) $\mathfrak{D} S(X)=\mathfrak{D} S_{c}(X)$.

Proof. It follows from Theorem 3.2 and [6, Corollary 4.6, Theorem 4.7 and Corollary 4.8]. 
In [8], L. C. Ciungu proved that for every pseudo-BCK(pDN) lattice the following conditions are equivalent (see [8, Proposition 3.5]):

$\left(\mathrm{P}_{1}\right)(x \wedge y) \rightarrow z=(x \rightarrow z) \vee(y \rightarrow z)$ and $(x \wedge y) \rightsquigarrow z=(x \rightsquigarrow z) \vee(y \rightsquigarrow z)$;

$\left(\mathrm{P}_{2}\right) z \rightarrow(x \vee y)=(z \rightarrow x) \vee(z \rightarrow y)$ and $z \rightsquigarrow(x \vee y)=(z \rightsquigarrow x) \vee(z \rightsquigarrow y)$

Also, she showed that the class of pseudo-BCK $(\mathrm{pDN})$ lattices satisfies the conditions $\left(\mathrm{P}_{1}\right)$ and $\left(\mathrm{P}_{2}\right)$ is not empty, since every pseudo-MV algebra satisfies these conditions. Further, It was proved that if a pseudo-BCK $(\mathrm{pDN})$ lattice $\mathfrak{X}$ satisfying $\left(\mathrm{P}_{1}\right)$ or $\left(\mathrm{P}_{2}\right)$, then $(X ; \preceq)$ is a distributive lattice (see $[8$, Theorem 3.4, Corollary 3.2]).

Theorem 3.3. Let $\mathfrak{S}=\left(S ; \vee_{1}, \vee_{2},\left({ }^{\rightarrow a}\right)_{a \in S},\left({ }^{\rightsquigarrow a}\right)_{a \in S}, 1\right)$ be a structure algebraic, where $\vee_{1}$ and $\vee_{2}$ are binary operations on $S$ and for each $a \in S, \rightarrow a$ and ${ }^{\rightsquigarrow a}$ are unary operations on $\left\{x \in S: a \vee_{1} x=1\right.$ and $\left.a \vee_{2} x=1\right\}$ and 1 is a distinguished element of $S$, satisfying the quasi-identities (1)-(12) from Theorem 3.1. Define the new binary operations $\rightarrow$ and $\rightsquigarrow$ on $S$ by

(C) $x \rightarrow y=\left(x \vee_{1} y\right)^{\rightarrow y}$ and $x \rightsquigarrow y=\left(x \vee_{2} y\right)^{\rightsquigarrow y}$.

Then $\mathfrak{X}(\mathfrak{S})=(S ; \rightarrow, \rightsquigarrow, 1)$ is a pseudo-BCK algebra.

Proof. The definition of $\rightarrow$ and $\rightsquigarrow$ are well define from Theorem 3.1 (8). Furthermore, we note that

(D) $x \vee_{1} y=y$ and $x \vee_{2} y=y$ if and only if $x \rightarrow y=1$ and $x \rightsquigarrow y=1$.

Indeed, if $x \vee_{1} y=y$, then $x \rightarrow y=\left(x \vee_{1} y\right)^{\rightarrow y}=y^{\rightarrow y}=1$, by Theorem 3.1 (2). Similarly, if $x \vee_{2} y=y$, then $x \rightsquigarrow y=\left(x \vee_{2} y\right)^{\rightsquigarrow y}=y^{\rightsquigarrow y}=1$.

For conversely, $1=x \rightarrow y=\left(x \vee_{1} y\right)^{\rightarrow y}$ implies $y=1^{\rightsquigarrow y}=\left(x \vee_{1} y\right)^{\rightarrow y \rightsquigarrow y}=x \vee_{1} y$. Also, $1=x \rightsquigarrow y=\left(x \vee_{2} y\right)^{\rightsquigarrow y}$ implies $y=1^{\rightarrow y}=\left(x \vee_{2} y\right)^{\rightsquigarrow y \rightarrow y}=x \vee_{2} y$, by Theorem 3.1 (3) and (10). Now, we verify the axioms of pseudo-BCK algebras as follows.

(psBCI $\left.)_{1}\right)$ Using Theorem 3.1 (5) and (12), we obtain

$$
\begin{aligned}
(x \rightarrow y) \vee_{2}\left(\left(x \vee_{1} z\right) \rightarrow\left(y \vee_{1} z\right)\right) & =\left(x \vee_{1} y\right)^{\rightarrow y} \vee_{2}\left(\left(x \vee_{1} z\right) \vee_{1}\left(y \vee_{1} z\right)\right) \rightarrow\left(y \vee_{1} z\right) \\
& =\left(\left(x \vee_{1} z\right) \vee_{1}\left(y \vee_{1} z\right)\right) \rightarrow\left(y \vee_{1} z\right) \\
& =\left(x \vee_{1} z\right) \rightarrow\left(y \vee_{1} z\right)
\end{aligned}
$$

Thus, $(x \rightarrow y) \rightsquigarrow\left(\left(x \vee_{1} z\right) \rightarrow\left(y \vee_{1} z\right)\right)=1$.

Also, according to Theorem 3.1 (10) and (11), we get

$$
(x \rightarrow y) \rightsquigarrow y=\left(\left(x \vee_{1} y\right)^{\rightarrow y} \vee_{2} y\right)^{\rightsquigarrow y}=x \vee_{1} y
$$

and

$$
x \rightarrow(y \rightsquigarrow z)=\left(x \vee_{1}\left(y \vee_{2} z\right)^{\rightsquigarrow z}\right)^{\rightarrow\left(y \vee_{2} z\right)}=\left(y \vee_{2}\left(x \vee_{1} z\right)^{\rightarrow z}\right)^{\rightsquigarrow\left(x \vee_{1} z\right)}=y \rightsquigarrow(x \rightarrow z)
$$

Then

$$
\begin{aligned}
\left(x \vee_{1} z\right) \rightarrow\left(y \vee_{1} z\right) & =((x \rightarrow z) \rightsquigarrow z) \rightarrow((y \rightarrow z) \rightsquigarrow z) \\
& =(y \rightarrow z) \rightsquigarrow(((x \rightarrow z) \rightsquigarrow z) \rightarrow z) \\
& =(y \rightarrow z) \rightsquigarrow(x \rightarrow z),
\end{aligned}
$$


since

$$
\begin{aligned}
((x \rightarrow z) \rightarrow z) \rightsquigarrow z & =\left(\left(\left(x \vee_{1} z\right)^{\rightarrow z} \vee_{2} z\right)^{\rightarrow z} \vee_{1} z\right)^{\rightarrow z} \\
& =\left(\left(x \vee_{1} z\right) \vee_{1} z\right)^{\rightarrow z} \\
& =\left(x \vee_{1} z\right)^{\rightarrow z} \\
& =x \rightarrow z
\end{aligned}
$$

Altogether, we have

$$
(x \rightarrow y) \rightsquigarrow((y \rightarrow z) \rightsquigarrow(x \rightarrow z))=(x \rightarrow y) \rightsquigarrow\left(\left(x \vee_{1} z\right) \rightarrow\left(y \vee_{1} z\right)\right)=1 .
$$

The second part of axiom ( $\left.\mathrm{psBCI}_{1}\right)$ follows by duality.

$\left(\mathrm{psBCI}_{2}\right)$ Using Theorem 3.1 (10), we get

$$
(x \rightarrow y) \rightsquigarrow y=\left(\left(x \vee_{1} y\right)^{\rightarrow y} \vee_{2} y\right)^{\rightsquigarrow y}=x \vee_{1} y .
$$

Hence, $x \vee_{1}((x \rightarrow y) \rightsquigarrow y)=x \vee_{1}\left(x \vee_{1} y\right)=x \vee_{1} y=(x \rightarrow y) \rightsquigarrow y$. Then $x \rightarrow((x \rightarrow y) \rightsquigarrow y)=1$.

By a similar argument we have $x \rightsquigarrow((x \rightsquigarrow y) \rightarrow y)=1$.

( $\mathrm{psBCI}_{3}$ ) Applying (D), from $x \vee_{1} x=x \vee_{2} x=x$ it follows $x \rightarrow x=x \rightsquigarrow x=1$.

$\left(\mathrm{psBCI}_{4}\right)$ If $x \rightarrow y=1$ and $y \rightarrow x=1$, then by Theorem 3.1 (6), we have $x \vee_{1} y=y$ and $y \vee_{1} x=x$. Now, using Theorem 3.1 (7), it follows $x=y$.

$\left(\mathrm{psBCI}_{5}\right)$ This follows from Theorem 3.1 (6) and (D).

(psBCK) By Theorem 3.1 (1), $x \vee_{1} 1=x \vee_{2} 1=1$. From (D) we see that $x \rightarrow 1=$ $x \rightsquigarrow 1=1$.

Theorem 3.4. Let $\mathfrak{S}=\left(S ; \vee_{1}, \vee_{2},(\rightarrow a)_{a \in S},\left({ }^{\sim a}\right)_{a \in S}, 1\right)$ be an algebra as in Theorem 3.3 satisfying (1)-(12) of Theorem 3.1 and $\mathfrak{X}$ be a pseudo-BCK algebra. Then $\mathfrak{X}(\mathfrak{S}(\mathfrak{X}))=\mathfrak{X}$ and $\mathfrak{S}(\mathfrak{X}(\mathfrak{S}))=\mathfrak{S}$.

Proof. By Theorem 3.1, $\mathfrak{S}(\mathfrak{X})=\left(X ; \vee_{1}, \vee_{2},\left({ }^{\rightarrow a}\right)_{a \in X},\left({ }^{\rightsquigarrow a}\right)_{a \in X}, 1\right)$ is the structure satisfying (1)-(12) which is assigned to a given pseudo-BCK algebra $\mathfrak{X}$. Then in $\mathfrak{X}(\mathfrak{S}(\mathfrak{X}))=\left(X ; \rightarrow_{1}, \rightsquigarrow_{1}, 1\right)$ we have

$$
x \rightarrow_{1} y=\left(x \vee_{1} y\right)^{\rightarrow y}=((x \rightarrow y) \rightsquigarrow y) \rightarrow y=x \rightarrow y
$$

and

$$
x \rightsquigarrow_{2} y=\left(x \vee_{2} y\right)^{\rightsquigarrow y}=((x \rightsquigarrow y) \rightarrow y) \rightsquigarrow y=x \rightsquigarrow y .
$$

Therefore, $\mathfrak{X}(\mathfrak{S}(\mathfrak{X}))=\mathfrak{X}$.

Also, assume that $\mathfrak{S}=\left(S ; \vee_{1}, \vee_{2},\left({ }^{\rightarrow a}\right)_{a \in S},\left({ }^{\cdots a}\right)_{a \in S}, 1\right)$ is a structure that satisfies (1)-(12) of Theorem 3.1, $\mathfrak{X}(\mathfrak{S})=(S ; \rightarrow, \rightsquigarrow, 1)$ its corresponding pseudo-BCK algebra (cf. Theorem 3.3) and $\mathfrak{S}(\mathfrak{X}(\mathfrak{S}))=\left(S ; \sqcup_{1}, \sqcup_{2},\left(r_{1 a}\right)_{a \in S},\left(r_{2 a}\right)_{a \in S}, 1\right)$. Then

$$
x \sqcup_{1} y=(x \rightarrow y) \rightsquigarrow y=\left(\left(x \vee_{1} y\right)^{\rightarrow y} \vee_{2} y\right)^{\rightsquigarrow y}=x \vee_{1} y
$$

and

$$
x \sqcup_{2} y=(x \rightsquigarrow y) \rightarrow y=\left(\left(x \vee_{2} y\right)^{\rightsquigarrow y} \vee_{1} y\right)^{\rightarrow y}=x \vee_{2} y .
$$


Further, for $x \in[a, 1]$, we have

$$
r_{1 a}(x)=x \rightarrow a=\left(x \vee_{1} a\right)^{\rightarrow a}=\left(\left(a \vee_{1} x\right) \vee_{1} a\right)^{\rightarrow a}=\left(a \vee_{1} x\right)^{\rightarrow a}=x^{\rightarrow a}
$$

and

$$
r_{2 a}(x)=x \rightsquigarrow a=\left(x \vee_{2} a\right)^{\rightsquigarrow a}=\left(\left(a \vee_{2} x\right) \vee_{2} a\right)^{\rightsquigarrow a}=\left(a \vee_{2} x\right)^{\rightsquigarrow a}=x^{\rightsquigarrow a} .
$$

Therefore, $\mathfrak{S}(\mathfrak{X}(\mathfrak{S}))=\mathfrak{S}$.

Corollary 3.3. Let $\mathfrak{S}=\left(S ; \vee_{1}, \vee_{2},(\rightarrow a)_{a \in S},\left({ }^{\rightsquigarrow a}\right)_{a \in S}, 1\right)$ be an algebraic structure satisfying (1)-(13) of Theorem 3.1. Then the relation defined by $(B)$ is a partial order on $S, 1$ is the greatest element of $S$ and for every $x, y \in S, x, y \preceq x \vee y$, where $\vee=\vee_{1}=\vee_{2}$. Moreover, for each $a \in S, \rightarrow a$ and ${ }^{\rightsquigarrow a}$ are antitone mappings on $[a, 1]=\{x \in S: a \preceq x\}$.

\section{CONCLUSION}

We consider that this paper could contribute to the study of algebraic structures and to the development of pseudo-BCK algebras. So, we hope it would be served as a foundation and another topic of research to define and investigate among algebraic structures derived from pseudo-BCK algebras. As another direction of research, one could investigate relationship between commutative pseudo-valuation on pseudo-BCK algebras with directoids.

Acknowledgements. The author would like to express their sincere thanks to the referees for the valuable suggestions which lead to an improvement of this paper. Support of the research by Payame Noor University, the office of Vice Chancellor for Research, Grant No. 49103/7, is gratefully acknowledged.

\section{REFERENCES}

[1] R. A. Borzooei, A. B. Saeid, A. Rezaei, A. Radfar and R. Ameri, On pseudo-BE algebras, Discuss. Math. Gen. Algebra Appl. 33 (2013), 95-108.

[2] R. A. Borzooei, A. B. Saeid, A. Rezaei, A. Radfar and R. Ameri, Distributive pseudo-BE algebras, Fasc. Math. 54 (2015), 21-39.

[3] I. Chajda and J. Kühr, Algebraic structures derived from BCK-algebras, Miskolc Math. Notes 8(1) (2007), 11-21.

[4] I. Chajda and H. Länger, An ordered structure of pseudo-BCI algebras, Math. Bohem. 141(1) (2016), 91-98.

[5] L. C. Ciungu, Commutative pseudo-BE algebras, Iran. J. Fuzzy Syst. 13(1) (2016), 131-144.

[6] L. C. Ciungu, Commutative deductive systems of pseudo BCK-algebras, Soft Computing 22(4) (2018), 1189-1201.

[7] L. C. Ciungu, Non-Commutative Multiple-Valued Logic Algebras, Springer, Cham, Heidelberg, New York, Dordrecht, London, 2014.

[8] L. C. Ciungu, On pseudo-BCK algebras with pseudo-double negation, An. Univ. Craiova Ser. Mat. Inform. 37(1) (2010), 19-26.

[9] W. A. Dudek and Y. B. Jun, Pseudo-BCI algebras, East Asian Math. J. 24 (2008), 187-190.

[10] G. Georgescu and A. Iorgulescu, Pseudo-MV algebras, Multiple-Valued Logic 9 (2001), 95-135. 
[11] G. Georgescu and A. Iorgulescu, Pseudo-BL algebras: a non-commutative extension of BLalgebras, Fifth International Conference FSTA 2000, Slovakia, February, 2000, 90-92.

[12] G. Georgescu and A. Iorgulescu, Pseudo-BCK Algebras: an Extension of BCK-Algebras, DMTCS01: Combinatorics, Computability and Logic, Springer, London, 2001, 97-114.

[13] G. Georgescu, L. Leustean and V. Preoteasa, Pseudo-hoops, J. Mult.-Valued Logic Soft Comput. 11 (2005), 153-184.

[14] R. Halaš and J. Kühr, Deductive systems and annihilators of pseudo-BCK algebras, Italian Journal of Pure and Applied Mathematics 25 (2009), 83-94.

[15] Y. Imai and K. Iséki, On axiom systems of propositional calculi, Proc. Japan Acad. 42 (1966), 19-22.

[16] A. Iorgulescu, Classes of pseudo-BCK algebras - Part I, J. Mult.-Valued Logic Soft Comput. 12 (2006), 71-130.

[17] A. Iorgulescu, Algebras of Logic as BCK-Algebras, Academy of Economic Studies Press, Bucharest, 2008.

[18] A. Iorgulescu, Implicative vs. Groups and Generalizations, Matrix Rom, Bucharest, 2018.

[19] K. Iséki, An algebra related with a propositional calculus, Proc. Japan Acad. 42 (1966), 26-29.

[20] J. Kühr, Pseudo-BCK semilattices, Demonstr. Math. 40 (2007), 495-516.

[21] J. Kühr, Pseudo-BCK Algebras and Related Structures, Univerzita Palackeho v Olomouci, Olomouci, 2007.

[22] J. Kühr, Commutative pseudo-BCK algebras, Southeast Asian Bull. Math. 33 (2009), 451-475.

[23] J. Meng and Y. B. Jun, BCK-Algebras, Kyung Moon Sa Co., Seoul, Korea, 1994.

[24] J. Rachůnek, A non commutative generalization of $M V$-algebras, Czechoslovak Math. J. 52(2) (2002), 255-273.

[25] A. Rezaei, A new trend to pseudo-BCK algebras, in: Proceeding of $6^{\text {th }}$ Iranian Joint Congress on Fuzzy and Intelligent Systems (CFIS), Kerman, Iran, 2018, 117-119.

[26] A. Rezaei, A. B. Saeid, A. Radfar and R. A. Borzooei, Congruence relations on pseudo-BE algebras, An. Univ. Craiova Ser. Mat. Inform. 41 (2014), 166-176.

[27] A. Rezaei, L. C. Ciungu and A. B. Saeid, States on pseudo-BE algebras, J. Mult.-Valued Logic Soft Comput. 28 (2017), 591-618.

[28] A. Rezaei, A. B. Saeid and K. Y. S. Saber, On pseudo-CI algebras, Soft Computing 23(13) (2019), 4643-4654.

[29] A. Rezaei, A. B. Saeid and A. Walendziak, Some results on pseudo-Q algebras, Ann. Univ. Paedagog. Crac. Stud. Math. 16 (2017), 61-72.

[30] A. Rezaei, A. Walendziak and A. B. Saeid, Some remarks on commutative and pointed pseudo-CI algebras, Mathematica Aeterna 8(4) (2018), 269-277.

[31] S. Tanaka, A new class of algebras, Mathematical Seminar Notes 3 (1975), 37-43.

[32] A. Walendziak, Pseudo-BCH algebras, Discuss. Math. Gen. Algebra Appl. 35 (2015), 5-19.

[33] A. Walendziak, On branchwise commutative pseudo-BCH algebras, Ann. Univ. Mariae CurieSklodowska Sect. A LXXI(2) (2017), 79-89.

${ }^{1}$ Department of Mathematics,

PAyAme Noor University,

P. O. Box. 19395-3697, TEHran, IrAN

Email address: rezaei@pnu.ac.ir 

Kragujevac Journal of Mathematics

Volume 46(1) (2022), Pages 139-148.

\title{
A NOTE ON COMPARISON OF ANNULI CONTAINING ALL THE ZEROS OF A POLYNOMIAL
}

\author{
SUNIL HANS ${ }^{1}$, AMIT TOMAR ${ }^{1}$, AND JIANHENG CHEN ${ }^{2}$
}

\begin{abstract}
If $P(z)$ is a polynomial of degree $n$, then for a subclass of polynomials, Dalal and Govil [7] compared the bounds, containing all the zeros, for two different results with two different real sequences $\lambda_{k}>0, \sum_{k=1}^{n} \lambda_{k}=1$. In this paper, we prove a more general result, by which one can compare the bounds of two different results with the same sequence of real or complex $\lambda_{k}, \sum_{k=0}^{n}\left|\lambda_{k}\right| \leq 1$. A variety of other results have been extended in this direction, which in particular include several known extensions and generalizations of a classical result of Cauchy [4], from this result by a fairly uniform manner.
\end{abstract}

\section{INTRODUCTION}

Properties of polynomials and especially the location of zeros is an old subject and it has been studied since the time of Gauss and Cauchy, as it finds important application in many areas of applied mathematics. Quite a few results giving the bound for some and all the zeros of a polynomial in terms of its coefficients can be found in [13-15].

Gauss was the earliest contributor to the study of location of zeros of a polynomial, who proved that a $n^{\text {th }}$ degree polynomial

$$
P(z):=a_{n} z^{n}+a_{n-1} z^{n-1}+\cdots+a_{1} z+a_{0},
$$

with all real $a_{k}$, has no zeros outside the circle $|z|=R$, where

$$
R=\max _{1 \leq k \leq n}\left(n \sqrt{2}\left|a_{k}\right|\right)^{1 / k} .
$$

Cauchy [4] improved the above result of Gauss by proving the following result.

Key words and phrases. Polynomial, zeros, Fibonacci's numbers.

2010 Mathematics Subject Classification. Primary: 30C15. Secondary: 26C10.

DOI 10.46793/KgJMat2201.139H

Received: November 16, 2018.

Accepted: September 11, 2019. 
Theorem 1.1. Let $P(z)=\sum_{k=0}^{n} a_{k} z^{k}$ be a complex polynomial of degree $n$. Then all the zeros of $P(z)$ lie in the disc

$$
\{z:|z| \leq r\} \subset\{z:|z|<1+M\}
$$

where

$$
M:=\max _{0 \leq k \leq n-1} \frac{\left|a_{k}\right|}{\left|a_{n}\right|}
$$

and $r$ is the unique positive real root of the real coefficients equation

$$
\left|a_{n}\right| z^{n}-\left|a_{n-1}\right| z^{n-1}-\left|a_{n-2}\right| z^{n-2}-\cdots-\left|a_{1}\right| z-\left|a_{0}\right|=0,
$$

when $P(z)$ is not a monomial, and as zero otherwise.

On applying the above theorem to the polynomial $Q(z)=z^{n} P(1 / z)$ and combining it with the above theorem, one can easily get the following theorem.

Theorem 1.2 (Cauchy). All the zeros of a $n^{\text {th }}$ degree polynomial $P(z)=\sum_{k=0}^{n} a_{k} z^{k}$ lie in the annulus $r_{1} \leq|z| \leq r_{2}$, where $r_{1}$ is the unique positive real root of the equation

$$
\left|a_{n}\right| z^{n}+\left|a_{n-1}\right| z^{n-1}+\cdots+\left|a_{1}\right| z-\left|a_{0}\right|=0,
$$

and $r_{2}$ is the unique positive real root of the equation

$$
\left|a_{n}\right| z^{n}-\left|a_{n-1}\right| z^{n-1}-\cdots-\left|a_{1}\right| z-\left|a_{0}\right|=0,
$$

when $P(z)$ is not a monomial, and as zero otherwise.

The above result of Cauchy has been sharpened among others by Joyal et al. [11], Datt and Govil [8], Affane-Aji et al. [1], and Sun and Hsieh [16].

Diaz-Barrero [9] proved the following result, which provides an annulus containing all the zeros of a polynomial by involving the Fibonacci numbers and binomial coefficients.

Theorem 1.3. Let $P(z)=\sum_{k=0}^{n} a_{k} z^{k}, a_{k} \neq 0,1 \leq k \leq n$, be a non-constant polynomial with complex coefficients. Then all its zeros lie in the annulus $\mathcal{C}=$ $\left\{z: r_{1} \leq|z| \leq r_{2}\right\}$, where

$$
r_{1}=\frac{3}{2} \min _{1 \leq k \leq n}\left(\frac{2^{n} F_{k} C(n, k)}{F_{4 n}}\left|\frac{a_{0}}{a_{k}}\right|\right)^{1 / k}
$$

and

$$
r_{2}=\frac{2}{3} \max _{1 \leq k \leq n}\left(\frac{F_{4 n}}{2^{n} F_{k} C(n, k)}\left|\frac{a_{n-k}}{a_{n}}\right|\right)^{1 / k} .
$$

Here $C(n, k)$ is the binomial coefficient and $F_{k}$ is the $k^{\text {th }}$ Fibonacci number.

Kim [12] used the identity $\sum_{k=0}^{n} C(n, k)=2^{n}$ to prove the following result. 
Theorem 1.4. Let $P(z)=\sum_{k=0}^{n} a_{k} z^{k}, a_{k} \neq 0,1 \leq k \leq n$, be a non-constant polynomial with complex coefficients. Then all the zeros of $P(z)$ lie in the annulus $\mathcal{C}=\left\{z: r_{1} \leq|z| \leq r_{2}\right\}$, where

$$
r_{1}=\min _{1 \leq k \leq n}\left(\frac{C(n, k)}{2^{n}-1}\left|\frac{a_{0}}{a_{k}}\right|\right)^{1 / k}
$$

and

$$
r_{2}=\max _{1 \leq k \leq n}\left(\frac{2^{n}-1}{C(n, k)}\left|\frac{a_{n-k}}{a_{n}}\right|\right)^{1 / k} .
$$

Here $C(n, k)$ is the binomial coefficient.

There are many results, including the above Theorems 1.3 and 1.4, available in this direction (for example, see $[3,5,10]$ ).

Aziz and Qayoom [2] (see also [5]) proved the following theorem which generalizes all the above results.

Theorem 1.5. Let $\lambda_{1}, \lambda_{2}, \ldots, \lambda_{n}$ be any set of $n$ real or complex numbers such that $\sum_{k=1}^{n}\left|\lambda_{k}\right| \leq 1$, and let $P(z)=\sum_{k=0}^{n} a_{k} z^{k}, a_{k} \neq 0,1 \leq k \leq n$, be a non-constant polynomial with complex coefficients. Then all the zeros of $P(z)$ lie in the annulus $\mathcal{C}=\left\{z: r_{1} \leq|z| \leq r_{2}\right\}$, where

$$
r_{1}=\min _{1 \leq k \leq n}\left|\lambda_{k} \frac{a_{0}}{a_{k}}\right|^{1 / k}
$$

and

$$
r_{2}=\max _{1 \leq k \leq n}\left|\frac{1}{\lambda_{k}} \frac{a_{n-k}}{a_{n}}\right|^{1 / k} .
$$

Theorem 1.5 can generate infinitely many results, including Theorem 1.2 to 1.4, giving an annulus containing all the zeros of a polynomial, and over the years, mathematicians were comparing the bounds with the existing bounds in the literature by giving some examples. Dalal and Govil [6] have shown that these bounds cannot, in general, be compared, implying that every result obtained can be useful. More recently, Dalal and Govil [7] successfully compared the bounds of two different results with two different real sequences $\lambda_{k}>0, \sum_{k=0}^{n} \lambda_{k}=1$ for a subclass of polynomials.

The main aim of this paper is to prove the following more general results (Theorem 2.1, 2.2) of Section 2 using well known Hölder Inequality

$$
\sum_{k=1}^{n} \alpha_{k} \beta_{k} \leq\left(\sum_{k=1}^{n} \alpha_{k}^{q}\right)^{1 / q}\left(\sum_{k=1}^{n} \beta_{k}^{p}\right)^{1 / p},
$$

where $\alpha_{k}>0, \beta_{k}>0$ for all $1 \leq k \leq n$, and $p>1, q>1$, with $1 / p+1 / q=1$, by which one can easily compare the bounds of two different results with the same sequence of real or complex $\lambda_{k}, \sum_{k=0}^{n}\left|\lambda_{k}\right| \leq 1$. Our results not only extend Theorem 1.5 but also 
improve it. Besides that theorems like Theorem 1.3, 1.4 and other similar to them also extended and shown as corollaries of Theorem 2.1 in the Table 1.

As above mentioned, the annuli obtained by Theorem 2.1 of Section 2 and Theorem 1.5 cannot, in general, be compared, however under some condition by MATLAB, we construct example of polynomial, for which the bounds obtained by our Theorem 2.1 and 2.2 are considerably sharper than the bounds obtained by Theorem 1.5. This has been done in Section 4.

\section{Main Results}

Theorem 2.1. Let $\lambda_{1}, \lambda_{2}, \ldots, \lambda_{n}$ be any set of $n$ real or complex numbers such that $\sum_{k=1}^{n}\left|\lambda_{k}\right| \leq 1$. For $p$ and $q$ such that $p>1, q>1, \frac{1}{p}+\frac{1}{q}=1$, the non-constant polynomial $P(z)=\sum_{k=0}^{n} a_{k} z^{k}, a_{k} \neq 0,1 \leq k \leq n$, with complex coefficients, has all its zeros lie in the annulus $\mathcal{C}=\left\{z: r_{1}^{\prime} \leq|z| \leq r_{2}^{\prime}\right\}$, where

$$
r_{1}^{\prime}=\min _{1 \leq k \leq n}\left|\frac{\lambda_{k}^{1 / p}}{n^{1 / q}} \frac{a_{0}}{a_{k}}\right|^{1 / k}
$$

and

$$
r_{2}^{\prime}=\max _{1 \leq k \leq n}\left|\frac{n^{1 / q}}{\lambda_{k}^{1 / p}} \frac{a_{n-k}}{a_{n}}\right|^{1 / k} .
$$

Remark 2.1. If for all $1 \leq k \leq n$, we take $\left|\lambda_{k}\right|=1 / n$, then by using $1 / p+1 / q=1$, we have from Theorem 2.1

$$
r_{1}^{\prime}=\min _{1 \leq k \leq n}\left|\frac{1}{n^{1 / p} n^{1 / q}} \frac{a_{0}}{a_{k}}\right|^{1 / k}=\min _{1 \leq k \leq n}\left|\frac{1}{n} \frac{a_{0}}{a_{k}}\right|^{1 / k}
$$

and

$$
r_{2}^{\prime}=\max _{1 \leq k \leq n}\left|n \frac{a_{n-k}}{a_{n}}\right|^{1 / k}
$$

which is similar to the bounds of Theorem 1.5 with $\left|\lambda_{k}\right|=1 / n, 1 \leq k \leq n$, but by Theorem 2.8 of Dalal and Govil [7], it is easy to see that if $\left|\lambda_{k}\right|<1 / n, 1 \leq k \leq n$, then Theorem 2.1 always gives a better bound than Theorem 1.5. Although, we are not able to prove analytically that whether any condition required over the coefficients of a polynomial or not, but with the help of MATLAB, we construct 1 million random polynomials, without any condition on coefficients, with different degrees, random $\lambda_{k}$, with $\left|\lambda_{k}\right|<1 / n$, and random $p>1, q>1$, with $1 / p+1 / q=1$, and that shows the bounds of our Theorem 2.1 is always better than Theorem 1.5. For reference, a result of a $30^{\text {th }}$ degree polynomial has been shown in Figure 2.1 below.

Remark 2.2. Let $r$ be the positive root of the equation

$$
z^{n p}\left|a_{n}\right|^{p}-z^{(n-1) p} n^{p / q}\left|a_{n-1}\right|^{p}-\cdots-z^{p} n^{p / q}\left|a_{1}\right|^{p}-n^{p / q}\left|a_{0}\right|^{p}=0 .
$$



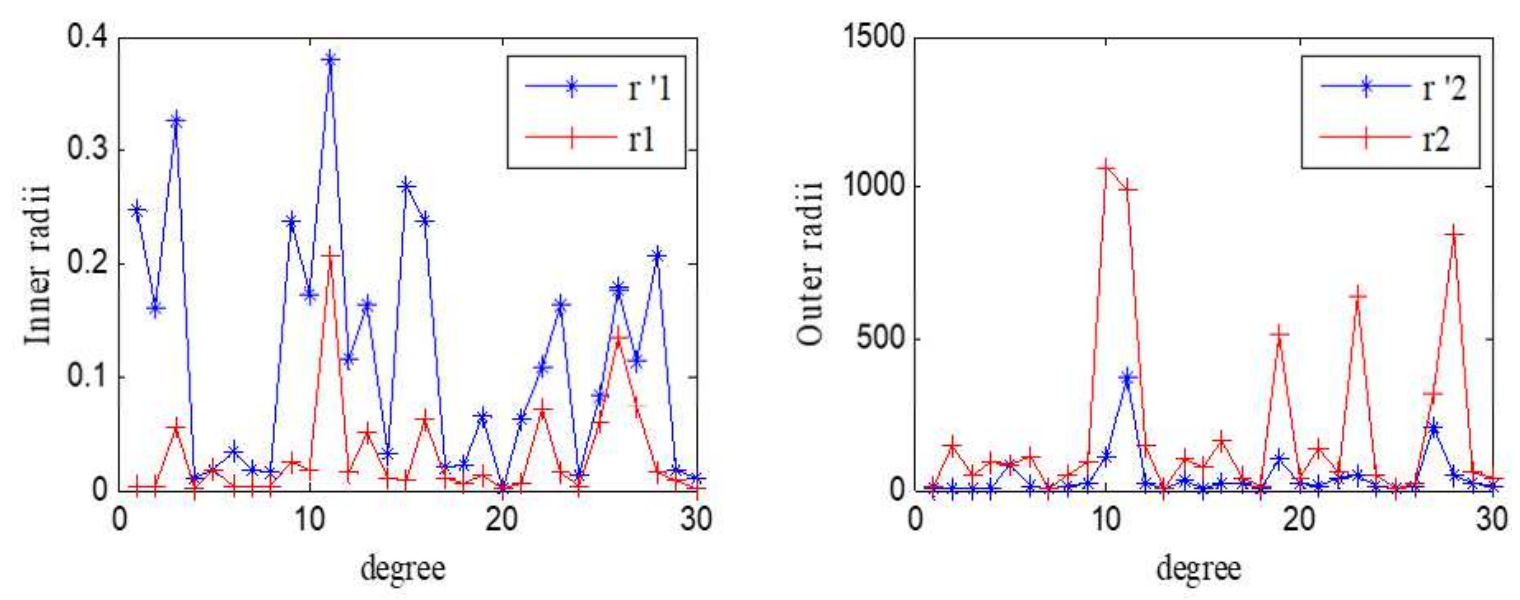

FiguRE 1. Comparison of bounds of Theorem 2.1 and Theorem 1.5

Therefore, we have

$$
\left\{\left|\frac{a_{0}}{a_{n}}\right|^{p} \frac{n^{p / q}}{r^{n p}}+\left|\frac{a_{1}}{a_{n}}\right|^{p} \frac{n^{p / q}}{r^{(n-1) p}}+\cdots+\left|\frac{a_{n-1}}{a_{n}}\right|^{p} \frac{n^{p / q}}{r^{p}}\right\}=1 .
$$

If we take

$$
\lambda_{k}=\left|\frac{a_{n-k}}{a_{n}}\right|^{p} \frac{n^{p / q}}{r^{k p}}, \quad k=1,2, \ldots, n,
$$

the equality (2.4) yields

$$
\sum_{k=1}^{n}\left|\lambda_{k}\right|=1
$$

Now, Theorem 2.1 implies that

$$
r_{2}=\max _{1 \leq k \leq n}\left\{\frac{n^{1 / q}}{\left|\lambda_{k}\right|^{1 / p}}\left|\frac{a_{n-k}}{a_{n}}\right|\right\}^{1 / k}=r .
$$

Hence, it follows that all the zeros of a polynomial $P(z)$ lie in $|z| \leq r$, where $r$ is the positive root of the equation (2.3). This generalizes Theorem 1.2 due to Cauchy [4], which is fairly deduced by our Theorem 2.1. The lower bound can be obtained in a similar way. Taking $q \rightarrow+\infty(p \rightarrow 1)$, this result reduces to Theorem 1.2.

Table 1 represents corollaries using some of the existing identities. The left column of the table contains the identities used in place of $\left|\lambda_{k}\right|$ in Theorem 2.1 and the right column shows the extension of corresponding existing results. So many other results can be obtained using different identities.

Remark 2.3. By (2.5) and (2.6), one can see that the best possible result this direction comes when $D_{r_{1}}^{\prime}:=1-\left(\sum_{k=1}^{n}\left|\frac{a_{k}}{a_{0}}\right|^{p} n^{p / q} r_{1}^{k p}\right)^{1 / p}=0$ and 
TABLE 1. Corollaries

\begin{tabular}{|l|l|}
\hline \multicolumn{1}{|c|}{$\left|\lambda_{k}\right|$} & \multicolumn{1}{|c|}{ Existing Results } \\
\hline 1. $\frac{2^{n-k} 3^{k} F_{k} C(n, k)}{F_{4 n}}, F_{k}$ is the $k^{\text {th }}$ Fibonacci number & Theorem 1.3 of $[9]$ \\
\hline 2. $\frac{C(n, k)}{2^{n}-1}, C(n, k)$ are the Binomial coefficients & Theorem 1.4 of $[12]$ \\
\hline 3. $\frac{C(n, k) A_{k} B_{j}^{k}\left(b B_{j-1}\right)^{n-k}}{A_{j n}}$ & Theorem 2 of [10] \\
\hline 4. $\frac{L_{k}}{L_{n+2}-3}, L_{k}$ is $k^{\text {th }}$ Lucas number & Corollary 2.1 of [5] \\
\hline 5. $\frac{C_{k-1} C_{n-k}}{C_{n}}, C_{k}$ is the $k^{\text {th }}$ Catalan number & Corollary 2.2 of [5] \\
\hline
\end{tabular}

$D_{r_{2}}^{\prime}:=1-\left(\sum_{k=1}^{n}\left|\frac{a_{n-k}}{a_{n}}\right|^{p} \frac{n^{p / q}}{r_{2}^{k p}}\right)^{1 / p}=0$. But, this result is implicit in the sense that in order to find the annulus containing all the zeros of a polynomial, we need to compute the roots of another associated equation (2.3). Our Theorem 2.1 provide a family of annuli that contains all the zeros of a polynomial based only on the polynomial coefficients.

Our next result improves upon any bound $r_{1}, r_{2}$ obtainable from any of the above mentioned results if $D_{r_{1}}^{\prime}>0$ and $D_{r_{2}}^{\prime}>0$.

Theorem 2.2. Let $\lambda_{1}, \lambda_{2}, \ldots, \lambda_{n}$ be any set of $n$ real or complex numbers such that $\sum_{k=1}^{n}\left|\lambda_{k}\right| \leq 1$. If $r_{1}$ and $r_{2}$ be any positive numbers such that $D_{r_{1}}^{\prime} \geq 0$ and $D_{r_{2}}^{\prime} \geq 0$, then for $p$ and $q$ such that $p>1, q>1, \frac{1}{p}+\frac{1}{q}=1$, the non-constant polynomial $P(z)=\sum_{k=0}^{n} a_{k} z^{k}, a_{k} \neq 0,1 \leq k \leq n$, has all its zeros lie in the annulus $\mathcal{C}=$ $\left\{z: r_{1}^{\prime \prime} \leq|z| \leq r_{2}^{\prime \prime}\right\}$, where

$$
r_{1}^{\prime \prime}=\min _{1 \leq k \leq n}\left(r_{1}^{k}+D_{r_{1}}^{\prime}\left|\frac{\lambda_{k}^{1 / p}}{n^{1 / q}} \frac{a_{0}}{a_{k}}\right|\right)^{1 / k}
$$

and

$$
r_{2}^{\prime \prime}=\max _{1 \leq k \leq n}\left(r_{2}^{-k}+D_{r_{2}}^{\prime}\left|\frac{\lambda_{k}^{1 / p}}{n^{1 / q}} \frac{a_{n}}{a_{n-k}}\right|\right)^{-1 / k} .
$$

Remark 2.4. (a) Since, it is clear that $r_{1}^{\prime \prime} \geq r_{1}$ and $r_{2}^{\prime \prime} \leq r_{2}$ therefore the Theorem 2.2 always gives bound sharper than any of the bound that has been obtained in terms of $r_{1}$ and $r_{2}$.

(b) In order to obtain better and better bound, Theorem 2.2 can be applied multiple times by substituting newly computed $r_{1}^{\prime \prime}$ and $r_{2}^{\prime \prime}$ as $r_{1}$ and $r_{2}$ in the formula, and making corresponding substitutions in $D_{r_{1}}^{\prime}$ and $D_{r_{2}}^{\prime}$ respectively and this has been shown in Section 4.

If we take $q \rightarrow+\infty(p \rightarrow 1)$ in the above Theorem 2.2 with $\lim _{q \rightarrow+\infty} D_{r_{1}}^{\prime}=$ $1-\sum_{k=1}^{n}\left|\frac{a_{k}}{a_{0}}\right| r_{1}^{k}=: D_{r_{1}}$ and $\lim _{q \rightarrow+\infty} D_{r_{2}}^{\prime}=1-\sum_{k=1}^{n}\left|\frac{a_{n-k}}{a_{n}}\right| \frac{1}{r_{2}^{k}}=: D_{r_{2}}$, we get the following corollary, which in particular gives a result due to Dalal and Govil [6]. 
Corollary 2.1. Let $\lambda_{1}, \lambda_{2}, \ldots, \lambda_{n}$ are any set of $n$ real or complex numbers such that $\sum_{k=1}^{m}\left|\lambda_{k}\right| \leq 1$. If $r_{1}$ and $r_{2}$ be any positive numbers such that $D_{r_{1}} \geq 0$ and $D_{r_{2}} \geq 0$, then the non-constant polynomial $P(z)=\sum_{k=0}^{n} a_{k} z^{k}, a_{k} \neq 0,1 \leq k \leq n$, has all its zeros lie in the annulus $\mathcal{C}=\left\{z: r_{1}^{\prime \prime} \leq|z| \leq r_{2}^{\prime \prime}\right\}$, where

$$
r_{1}^{\prime \prime}=\min _{1 \leq k \leq n}\left(r_{1}^{k}+D_{r_{1}}\left|\lambda_{k} \frac{a_{0}}{a_{k}}\right|\right)^{1 / k}
$$

and

$$
r_{2}^{\prime \prime}=\max _{1 \leq k \leq n}\left(r_{2}^{-k}+D_{r_{2}}\left|\lambda_{k} \frac{a_{n}}{a_{n-k}}\right|\right)^{-1 / k}
$$

\section{Proof of the Theorems}

Proof of Theorem 2.1. Proof of Theorem 2.1 follows on the same lines of Theorem 2.2, therefore we omit the details.

Proof of Theorem 2.2. If $a_{0}=0$, then $r_{1}^{\prime}=0$ which implies $r_{1}^{\prime \prime}=r_{1}^{\prime}=0$. Therefore, without loss of generality, we can suppose that $a_{0} \neq 0$. Let $z$ be such that $|z|<r_{1}^{\prime \prime}$. Then we have

$$
\begin{aligned}
|P(z)| & \geq\left|a_{0}\right|-\left|\sum_{k=1}^{n} a_{k} z^{k}\right| \geq\left|a_{0}\right|-\sum_{k=1}^{n}\left|a_{k}\right||z|^{k}>\left|a_{0}\right|-\sum_{k=1}^{n}\left|a_{k}\right|\left(r_{1}^{\prime \prime}\right)^{k} \\
& =\left|a_{0}\right|\left[1-\sum_{k=1}^{n}\left|\frac{a_{k}}{a_{0}}\right|\left(r_{1}^{\prime \prime}\right)^{k}\right] .
\end{aligned}
$$

Now, from (2.7), we get

$$
\left|\frac{a_{k}}{a_{0}}\right|\left(r_{1}^{\prime \prime}\right)^{k} \leq\left|\frac{a_{k}}{a_{0}}\right| r_{1}^{k}+D_{r_{1}}^{\prime} \frac{\left|\lambda_{k}\right|^{1 / p}}{n^{1 / q}}, \quad \text { for } k=1,2, \ldots, n .
$$

Substituting (3.2) in (3.1), and using Hölder inequality (1.3), we obtain

$$
\begin{aligned}
|P(z)| & >\left|a_{0}\right|\left[1-\sum_{k=1}^{n}\left|\frac{a_{k}}{a_{0}}\right| r_{1}^{k}-\sum_{k=1}^{n} D_{r_{1}}^{\prime} \frac{\left|\lambda_{k}\right|^{1 / p}}{n^{1 / q}}\right] \\
& \geq\left|a_{0}\right|\left[1-n^{1 / q}\left(\sum_{k=1}^{n}\left|\frac{a_{k}}{a_{0}}\right|^{p} r_{1}^{k p}\right)^{1 / p}-D_{r_{1}}^{\prime}\left(\sum_{k=1}^{n}\left|\lambda_{k}\right|\right)^{1 / p}\right] \\
& =\left|a_{0}\right|\left[D_{r_{1}}^{\prime}-D_{r_{1}}^{\prime}\left(\sum_{k=1}^{m}\left|\lambda_{k}\right|\right)^{1 / p}\right] \\
& =\left|a_{0}\right| D_{r_{1}}^{\prime}\left[1-\left(\sum_{k=1}^{m}\left|\lambda_{k}\right|\right)^{1 / p}\right] \geq 0
\end{aligned}
$$

as $\sum_{k=1}^{m}\left|\lambda_{k}\right| \leq 1$, and consequently $P(z)$ does not have any zero in $|z|<r_{1}^{\prime \prime}$. 
To prove the second part of the theorem, we shall use the first part. Consider the polynomial

$$
Q(z)=z^{n} P\left(\frac{1}{z}\right)=a_{0} z^{n}+a_{1} z^{n-1}+\cdots+a_{n-1} z+a_{n} .
$$

By the first part of the theorem, all the zeros of the polynomial $Q(z)$ lie in

$$
\begin{aligned}
|z| & \geq \min _{1 \leq k \leq n}\left(\frac{1}{r_{2}^{k}}+\frac{D_{r_{2}}^{\prime}\left|\lambda_{k}\right|^{1 / p}}{n^{1 / q}}\left|\frac{a_{n}}{a_{n-k}}\right|\right)^{1 / k} \\
& =\min _{1 \leq k \leq m} \frac{1}{\left(r_{2}^{-k}+\frac{D_{r_{2}}^{\prime}\left|\lambda_{k}\right|^{1 / p}}{n^{1 / q}}\left|\frac{a_{n}}{a_{n-k}}\right|\right)^{-1 / k}} \\
& =\frac{1}{\max _{1 \leq k \leq m}\left(r_{2}^{-k}+\frac{D_{r_{2}}^{\prime}\left|\lambda_{k}\right|^{1 / p}}{n^{1 / q}}\left|\frac{a_{n}}{a_{n-k}}\right|\right)^{-1 / k}}=\frac{1}{r_{2}^{\prime \prime}}
\end{aligned}
$$

Replacing $z$ by $\frac{1}{z}$ and observing that $P(z)=z^{n} Q\left(\frac{1}{z}\right)$, we conclude that all the zeros of $P(z)$ lie in

$$
|z| \leq r_{2}^{\prime \prime}=\max _{1 \leq k \leq m}\left(r_{2}^{-k}+\frac{D_{r_{2}}^{\prime}\left|\lambda_{k}\right|^{1 / p}}{n^{1 / q}}\left|\frac{a_{n}}{a_{n-k}}\right|\right)^{-1 / k}
$$

and (2.8) is thus established.

\section{NUMERICAL EXPERIMENTS}

By Figure 2.1, it is easy to see that for any random polynomial, random sequence of $\lambda_{k}, \sum_{k=0}^{n}\left|\lambda_{k}\right|<1 / n, 1 \leq k \leq n$ and random $p>1, q>1$, with $1 / p+1 / q=1$, our Theorem 2.1 always gives better bounds than Theorem 1.5. Here, we construct an example of a polynomial in order to compare our results, Theorems 2.1 and 2.2, with Theorem 1.5.

Example 4.1. Let $P(z)=z^{4}+0.01 z^{3}+0.1 z^{2}+0.2 z+0.4$.

TABLE 2. Comparison of Annuli (Theorem 2.2 applying again to $P(z)$ using the bounds $r_{1}=0.5187$ and $r_{2}=1.0628$ )

\begin{tabular}{lccc}
\hline Result & $r_{1}$ & $r_{2}$ & Area of annulus \\
\hline Theorem 1.5 & 0.2847 & 1.1647 & 4.0070 \\
Theorem 2.1 & 0.4343 & 1.1345 & 3.4509 \\
Theorem 2.2 & 0.5187 & 1.0628 & 2.7033 \\
Theorem 2.2 & 0.5539 & 1.0442 & 2.4615 \\
Actual bound & 0.7412 & 0.8532 & 0.5610 \\
\hline
\end{tabular}


In Table 2, we first apply Theorems 1.5 and 2.1 to $P(z)$ using the random sequence $\lambda_{k}, \sum_{k=0}^{n}\left|\lambda_{k}\right|<1 / n$ and random $p>1, q>1$, with $1 / p+1 / q=1$. We see that Theorem 2.1 gives sharper bounds than Theorem 1.5. We then apply Theorem 2.2 to $P(z)$ using the same sequence of $\lambda_{k}, p, q$ and the radii $r_{1}=0.4343$ and $r_{2}=1.1345$, and we obtain better bounds than Theorem 2.1. Applying again Theorem 2.2 to $P(z)$ using the radii $r_{1}=0.5187$ and $r_{2}=1.0628$ of Theorem 2.2 , we get new bounds $r_{1}^{\prime \prime}=0.5539$ and $r_{2}^{\prime \prime}=1.0442$, which are more closer to the actual radii of the annulus that contains all the zeros of $P(z)$.

Acknowledgements. The authors are extremely grateful to the referee for his valuable suggestions.

\section{REFERENCES}

[1] C. Affane-Aji, S. Biaz and N. K. Govil, On annuli containing all the zeros of a polynomial, Math. Comp. Modelling 52 (2010) 1532-1537.

[2] A. Aziz and A. Qayoom, Estimates for the modulii of the zeros of a polynomial, Math. Inequal. Appl. 9 (2006) 107-116.

[3] M. Bidkham and E. Shashahani, An annulus for the zeros of polynomials, Appl. Math. Lett. 24 (2011) 122-125.

[4] A. L. Cauchy, Exercises de Mathématiques, IV, Anné de Bure Fréres, Paris, 1829.

[5] A. Dalal and N. K. Govil, On region containing all the zeros of a polynomial, Appl. Math. Comput. 219 (2013) 9609-9614.

[6] A. Dalal and N. K. Govil, Annulus containing all the zeros of a polynomial, Appl. Math. Comput. 249 (2014) 429-435.

[7] A. Dalal and N. K. Govil, On comparison of annuli containing all the zeros of a polynomial, Appl. Anal. Discrete Math. 11 (2017) 232-241.

[8] B. Datt and N. K. Govil, On the location of zeros of a polynomial, J. Approx. Theory 24 (1978) $78-82$.

[9] J. L. Diaz-Barrero, An annulus for the zeros of polynomials, J. Math. Anal. Appl. 273 (2002) 349-352.

[10] J. L. Diaz-Barrero and J. J. Egozcue, Bounds for the moduli of zeros, Appl. Math. Lett. 17 (2004) 993-996.

[11] A. Joyal, G. Labelle and Q. I. Rahman, On the location of zeros of polynomials, Canad. Math. Bull. 10 (1967) 53-63.

[12] S. H. Kim, On the moduli of the zeros of a polynomial, Amer. Math. Monthly 112 (2005) 924-925.

[13] M. Marden, Geometry of Polynomials, 2nd edition, Mathematical Surveys 3, Amer. Math. Soc. Providence, RI, USA, 1966.

[14] G. V. Milovanović, D. S. Mitrinović and Th. M. Rassias, Topics in Polynomials: Extremal Problems, Inequalities and Zeros, World Scientific, Singapore, 1994.

[15] Q. I. Rahman and G. Schmeisser, Analytic Theory of Polynomials, Oxford University Press Inc., New York, 2002.

[16] Y. J. Sun and J. G. Hsieh, A note on circular bound of polynomial zeros, IEEE Trans. Circuits Syst. I. Regul. Pap. 43 (1996) 476-478. 
${ }^{1}$ Department of Applied Mathematics, AMITY University,

NoIDA-201313, INDIA

Email address: sunil.hans82@yahoo.com

Email address: atomar@amity.edu

${ }^{2}$ School of Mathematics and Computing Science, Guilin University of Electronic Technology, Guilin-541004, ChinA

Email address: cjh868@126.com 
Kragujevac Journal of Mathematics

Volume 46(1) (2022), Pages 149-165.

\title{
SOME RESULTS ON POST-WIDDER OPERATORS PRESERVING TEST FUNCTION $x^{r}$
}

\author{
VIJAY GUPTA ${ }^{1}$ AND GANCHO TACHEV ${ }^{2}$
}

\begin{abstract}
In the present paper, we consider Post-Widder operators and its modified form which preserve the test function $x^{r}, r \in \mathbb{N}$. We estimate direct results in terms of modulus of continuity for the modified operators. Also, some estimates for polynomially bounded functions and linear combinations are considered. Our estimates improve in some sense the previous results for the original Post-Widder operators.
\end{abstract}

\section{INTRODUCTION}

The Post-Widder operators for $n \in \mathbb{N}$ and $x>0$ considered by Widder [18] is defined by

$$
P_{n}(f, x)=\frac{1}{n !}\left(\frac{n}{x}\right)^{n+1} \int_{0}^{\infty} t^{n} e^{-\frac{n t}{x}} f(t) d t .
$$

These operators preserve constant functions only. The $q$ analogue of these operators was recently studied by Aydin et al. [3]. Earlier Rathore and Singh [15] (also for related results see [9]) established an asymptotic formula, and deduced inverse and saturation theorems in simultaneous approximation. They considered a parameter $p$ and defined the operators in following way

$$
P_{n}^{p}(f, x)=\frac{1}{(n+p) !}\left(\frac{n}{x}\right)^{n+p+1} \int_{0}^{\infty} t^{n+p} e^{-\frac{n t}{x}} f(t) d t .
$$

Key words and phrases. Post-Widder operators, exponential functions, moments, polynomially bounded functions, linear combinations.

2010 Mathematics Subject Classification. Primary: 41A25. Secondary: 41A36.

DOI $10.46793 / \mathrm{KgJMat} 2201.149 \mathrm{G}$

Received: May 15, 2019.

Accepted: September 19, 2019. 
The special case $p=0$ provides the operator (1.1) and for $p=-1$, these operators reduce to the operators due to May [13], which preserve the linear functions and considered earlier in the book of Ditizian and Totik [5], by Li and Wang in [12], also in the papers of Draganov and Ivanov [6,7]. Rempulska and Skorupka in [16] further modified the Post-Widder operators of the form considered by May [13] in order to preserve the test function $e_{2}$, where $e_{r}(x)=x^{r}$. It was observed in [16] that the modified form provide better approximation results over the form of [13], but in that case the modified form loses the preservation of test function $e_{1}$. It may be observed that at a time only two preservations can be made either constant and $e_{1}$ or constant and the function $e_{r}, r>1, r \in \mathbb{N}$. Here we deal with the modification of Post-Widder operators which preserve constants and $e_{r}, r \in \mathbb{N}$.

Following [16], the $r$-th order moment $\mu_{r}^{P_{n}}(x)=P_{n}\left(e_{r}, x\right)$, where $e_{r}(t)=t^{r}, r \in$ $\mathbb{N} \cup\{0\}$ are given by

$$
P_{n}\left(e_{r}, x\right)=\frac{1}{n !}\left(\frac{n}{x}\right)^{n+1} \int_{0}^{\infty} t^{n} e^{-\frac{n t}{x}} t^{r} d t=\frac{1}{n !}\left(\frac{n}{x}\right)^{n+1} \int_{0}^{\infty} t^{n+r} e^{-\frac{n t}{x}} d t .
$$

Put $n t / x=u$ implying $(n / x) d t=d u$, thus

$$
\begin{aligned}
P_{n}\left(e_{r}, x\right) & =\frac{1}{n !}\left(\frac{n}{x}\right)^{n+1} \int_{0}^{\infty}\left(\frac{x}{n}\right)^{n+r+1} u^{n+r} e^{-u} d u \\
& =\frac{1}{n !}\left(\frac{x}{n}\right)^{r} \Gamma(n+r+1) \\
& =\frac{1}{n !}\left(\frac{x}{n}\right)^{r}(n+r) ! \\
& =\frac{(n+r)(n+r-1) \cdots(n+1) n !}{n !} \cdot \frac{x^{r}}{n^{r}}=\frac{(n+1)_{r} x^{r}}{n^{r}},
\end{aligned}
$$

where $(n)_{r}=n(n+1)(n+2) \cdots(n+r-1)$.

$$
\mu_{r}^{P_{n}}(x)=\frac{(n+1)_{r} x^{r}}{n^{r}}
$$

with $(n)_{0}=1$. If the central moments are denoted by $T_{m}^{P_{n}}(x)=P_{n}\left((t-x)^{m}, x\right)$, then

$$
\begin{aligned}
& T_{1}^{P_{n}}(x)=\frac{x}{n}, \\
& T_{2}^{P_{n}}(x)=\frac{(n+2) x^{2}}{n^{2}} .
\end{aligned}
$$

From the results given in [5, Ch. 9], denoting the space of all real valued continuous and bounded functions on $(0, \infty)$ by $C_{B}(0, \infty)$ for every $f \in C_{B}(0, \infty)$ and $\delta>0$ there holds

$$
|f(t)-f(x)| \leq \omega(f, \delta)\left(\frac{|t-x|}{\delta}+1\right)
$$


where $\omega(f, \cdot)$ is the first order modulus of continuity of $f$ defined by

$$
\omega(f, \delta)=\sup _{0<h \leq \delta, x \geq 0}|f(x+h)-f(x)| .
$$

Thus,

$$
\begin{aligned}
\left|P_{n}(f, x)-f(x)\right| & \leq \frac{1}{n !}\left(\frac{n}{x}\right)^{n+1} \int_{0}^{\infty} t^{n} e^{-\frac{n t}{x}}|f(t)-f(x)| d t \\
& \leq \frac{1}{n !}\left(\frac{n}{x}\right)^{n+1} \int_{0}^{\infty} t^{n} e^{-\frac{n t}{x}} \omega(f, \delta)\left(\frac{|t-x|}{\delta}+1\right) d t \\
& \leq \omega(f, \delta)\left(\frac{\sqrt{T_{2}^{P_{n}}(x)}}{\delta}+1\right) .
\end{aligned}
$$

Choosing $\delta=\sqrt{T_{2}^{P_{n}}(x)}$, we immediately get

$$
\left|P_{n}(f, x)-f(x)\right| \leq 2 \omega\left(f, \frac{\sqrt{n+2}}{n} x\right) .
$$

\section{Modified Post-Widder Operators Preserving $e_{r}$}

Let us consider that the Post-Widder operators preserve the test function $x^{r}, r \in \mathbb{N}$, then we start with the following form

$$
\widetilde{P}_{n, r}(f, x)=\frac{1}{n !}\left(\frac{n}{a_{n, r}(x)}\right)^{n+1} \int_{0}^{\infty} t^{n} e^{-\frac{n t}{a_{n}, r(x)}} f(t) d t .
$$

Here $\widetilde{P}_{n, r}$ preserves constants for any positive function $a_{n, r}(x)$. Then

$$
\begin{aligned}
x^{r}=\widetilde{P}_{n, r}\left(e_{r}, x\right) & =\frac{1}{n !}\left(\frac{n}{a_{n, r}(x)}\right)^{n+1} \int_{0}^{\infty} t^{n+r} e^{-\frac{n t}{a_{n, r}(x)}} d t \\
& =\frac{(n+r) !}{n !}\left(\frac{a_{n, r}(x)}{n}\right)^{r}=(n+1)_{r}\left(\frac{a_{n, r}(x)}{n}\right)^{r},
\end{aligned}
$$

implying

$$
a_{n, r}(x)=\frac{n x}{\left((n+1)_{r}\right)^{1 / r}} .
$$

Thus, our modified operators $\widetilde{P}_{n, r}, r \in \mathbb{N}, x \in(0, \infty)$, take the following form

$$
\widetilde{P}_{n, r}(f, x)=\int_{0}^{\infty} k_{n}(x, t) f(t) d t,
$$

where

$$
k_{n}(x, t)=\frac{1}{n !}\left[\frac{\left((n+1)_{r}\right)^{1 / r}}{x}\right]^{n+1} t^{n} e^{-\frac{t}{x}\left((n+1)_{r}\right)^{1 / r}},
$$


with $\widetilde{P}_{n, r}(f, 0)=f(0)$ which preserve the function $x^{r}$ and the constant function.

Following (1.2), the $m$-th order moments are given by

$$
\widetilde{P}_{n, r}\left(e_{m}, x\right)=\frac{(n+1)_{m}\left(a_{n, r}(x)\right)^{m}}{n^{m}}=\frac{(n+1)_{m}}{\left((n+1)_{r}\right)^{m / r}} x^{m} .
$$

Lemma 2.1. The first few images of monomials are given by

$$
\begin{aligned}
& \widetilde{P}_{n, r}\left(e_{0}, x\right)=1, \\
& \widetilde{P}_{n, r}\left(e_{1}, x\right)=\frac{(n+1)}{\left((n+1)_{r}\right)^{1 / r}} x, \\
& \widetilde{P}_{n, r}\left(e_{2}, x\right)=\frac{(n+1)(n+2)}{\left((n+1)_{r}\right)^{2 / r}} x^{2} .
\end{aligned}
$$

Remark 2.1. We may point out here that when $r=1$, then the operator $\widetilde{P}_{n, 1}$ preserves constant as well as linear functions. When $r=2$ these preserve constant and test function $x^{2}$.

Lemma 2.2. If we denote the central moment as $T_{m}^{\widetilde{P}_{n, r}}(x)=\widetilde{P}_{n, r}\left((t-x)^{m}, x\right)$, then we have the following recurrence relation:

$T_{m+1}^{\widetilde{P}_{n, r}}(x)=\frac{x^{2}}{\left((n+1)_{r}\right)^{1 / r}}\left[T_{m}^{\widetilde{P}_{n, r}}(x)\right]^{\prime}+\left[\frac{(n+1)}{\left((n+1)_{r}\right)^{1 / r}}-1\right] x T_{m}^{\widetilde{P}_{n, r}}(x)+\frac{m x^{2} T_{m-1}^{\widetilde{P}_{n, r}}(x)}{\left((n+1)_{r}\right)^{1 / r}}$.

In particular

$$
\begin{aligned}
T_{1}^{\widetilde{P}_{n, r}}(x)= & {\left[\frac{(n+1)}{\left((n+1)_{r}\right)^{1 / r}}-1\right] x, } \\
T_{2}^{\widetilde{P}_{n, r}}(x)= & {\left[\frac{(n+1)_{2}}{\left((n+1)_{r}\right)^{2 / r}}-2 \frac{(n+1)}{\left((n+1)_{r}\right)^{1 / r}}+1\right] x^{2}, } \\
T_{4}^{\widetilde{P}_{n, r}}(x)= & {\left[\frac{(n+1)_{4}}{\left((n+1)_{r}\right)^{4 / r}}-4 \frac{(n+1)_{3}}{\left((n+1)_{r}\right)^{3 / r}}+6 \frac{(n+1)_{2}}{\left((n+1)_{r}\right)^{2 / r}}-4 \frac{(n+1)}{\left((n+1)_{r}\right)^{1 / r}}+1\right] x^{4}, } \\
T_{6}^{\widetilde{P}_{n, r}}(x)= & {\left[\frac{(n+1)_{6}}{\left((n+1)_{r}\right)^{6 / r}}-6 \frac{(n+1)_{5}}{\left((n+1)_{r}\right)^{5 / r}}+15 \frac{(n+1)_{4}}{\left((n+1)_{r}\right)^{4 / r}}\right.} \\
& \left.-20 \frac{(n+1)_{3}}{\left((n+1)_{r}\right)^{3 / r}}+15 \frac{(n+1)_{2}}{\left((n+1)_{r}\right)^{2 / r}}-6 \frac{(n+1)}{\left((n+1)_{r}\right)^{1 / r}}+1\right] x^{6} .
\end{aligned}
$$

For any $r \in \mathbb{N}$ we have $T_{m}^{\widetilde{P}_{n, r}}(x)=O\left(n^{-[(m+1) / 2]}\right)$.

Proof. The kernel $k_{n}(x, t)$ of our modified operators $\widetilde{P}_{n, r}$, satisfy the following identity

$$
x^{2} \frac{\partial}{\partial x} k_{n}(x, t)=\left[\left(\left((n+1)_{r}\right)^{1 / r}\right) t-(n+1) x\right] k_{n}(x, t),
$$


we have

$$
\begin{aligned}
x^{2}\left[T_{m}^{\widetilde{P}_{n, r}}(x)\right]^{\prime}= & \int_{0}^{\infty} x^{2}\left[k_{n}(x, t)\right]_{x}^{\prime}(t-x)^{m} d t-m x^{2} T_{m-1}^{\widetilde{P}_{n, r}}(x) \\
= & \int_{0}^{\infty}\left[\left((n+1)_{r}\right)^{1 / r} t-(n+1) x\right] k_{n}(x, t)(t-x)^{m} d t-m x^{2} T_{m-1}^{\widetilde{P}_{n, r}}(x) \\
= & \left((n+1)_{r}\right)^{1 / r} T_{m+1}^{\widetilde{P}_{n, r}}(x)+\left[\left((n+1)_{r}\right)^{1 / r}-(n+1)\right] x T_{m}^{\widetilde{P}_{n, r}}(x) \\
& -m x^{2} T_{m-1}^{\widetilde{P}_{n, r}}(x) .
\end{aligned}
$$

This completes the proof of recurrence relation. From recurrence relation by induction on $m$, it is easy to verify that the magnitude of the central moments satisfy $T_{m}^{\widetilde{P}_{n, r}}(x)=$ $O\left(n^{-[(m+1) / 2]}\right)$ for any $r \in \mathbb{N}$. The other consequences follow from the recurrence relation.

We have the following observations for our modified operator, corresponding to the estimate (1.3).

Let us suppose that the operators preserve the test functions $e_{1}, e_{2}, e_{3}, e_{4}$ respectively, then, by Lemma 2.2 for every continuous and bounded function $f$ on $(0, \infty)$, we have the following estimates:

$$
\begin{aligned}
& \left|\widetilde{P}_{n, 1}(f, x)-f(x)\right| \leq 2 \omega\left(f, \frac{x}{\sqrt{n+1}}\right) \\
& \left|\widetilde{P}_{n, 2}(f, x)-f(x)\right| \leq 2 \omega\left(f, \sqrt{2} \sqrt{\left(1-\sqrt{\frac{n+1}{n+2}}\right)}\right) \\
& \left|\widetilde{P}_{n, 3}(f, x)-f(x)\right| \\
& \leq 2 \omega\left(f, \sqrt{\left(\frac{[(n+1)(n+2)]^{1 / 3}}{(n+3)^{2 / 3}}-2 \frac{(n+1)^{2 / 3}}{[(n+2)(n+3)]^{1 / 3}}+1\right) x}\right) \\
& \left|\widetilde{P}_{n, 4}(f, x)-f(x)\right| \\
& \leq 2 \omega\left(f, \sqrt{\left(\frac{[(n+1)(n+2)]^{1 / 2}}{[(n+3)(n+4)]^{1 / 2}}-2 \frac{(n+1)^{3 / 4}}{[(n+2)(n+3)(n+4)]^{1 / 4}}+1\right) x}\right) .
\end{aligned}
$$

If we compare the above results, with the estimate (1.3), we find that the error becomes smaller and monotonically decreasing for $n \in \mathbb{N}, x \in(0, \infty)$, till the preservation of the test function $e_{3}$ as the following is true for second order moments:

$$
\begin{aligned}
\frac{\sqrt{n+2}}{n} & \geq \frac{1}{\sqrt{n+1}} \geq \sqrt{2} \sqrt{\left(1-\sqrt{\frac{n+1}{n+2}}\right)} \\
& >\sqrt{\left(\frac{[(n+1)(n+2)]^{1 / 3}}{(n+3)^{2 / 3}}-2 \frac{(n+1)^{2 / 3}}{[(n+2)(n+3)]^{1 / 3}}+1\right)},
\end{aligned}
$$


TABLE 1. Table for approximation

\begin{tabular}{|c|c|c|c|c|}
\hline $\mathrm{n}$ & $\sqrt{T_{2}^{\widetilde{P}_{n, 1}}(x)}$ & $\sqrt{T_{2}^{\widetilde{P}_{n, 2}}(x)}$ & $\sqrt{T_{2}^{\widetilde{P}_{n, 3}}(x)}$ & $\sqrt{T_{2}^{\widetilde{P}_{n, 4}}(x)}$ \\
\hline 5 & $0.408248 \mathrm{x}$ & $0.385175 \mathrm{x}$ & $0.378018 \mathrm{x}$ & $0.381509 \mathrm{x}$ \\
\hline 15 & $0.250000 \mathrm{x}$ & $0.244367 \mathrm{x}$ & $0.242538 \mathrm{x}$ & $0.243903 \mathrm{x}$ \\
\hline 25 & $0.196116 \mathrm{x}$ & $0.193356 \mathrm{x}$ & $0.192451 \mathrm{x}$ & $0.193203 \mathrm{x}$ \\
\hline 35 & $0.166667 \mathrm{x}$ & $0.164961 \mathrm{x}$ & $0.164399 \mathrm{x}$ & $0.164890 \mathrm{x}$ \\
\hline 40 & $0.156174 \mathrm{x}$ & $0.154767 \mathrm{x}$ & $0.154303 \mathrm{x}$ & $0.154716 \mathrm{x}$ \\
\hline 75 & $0.114708 \mathrm{x}$ & $0.114147 \mathrm{x}$ & $0.113961 \mathrm{x}$ & $0.114135 \mathrm{x}$ \\
\hline 10000 & $0.0099995 \mathrm{x}$ & $0.00999913 \mathrm{x}$ & $0.0099990 \mathrm{x}$ & $0.00999913 \mathrm{x}$ \\
\hline
\end{tabular}

but for higher order preservation of test function for example preservation of test function $e_{4}$, one can not have better approximation, which is also shown in the above table. Although the convergence takes places in all the cases for $n$ sufficiently large.

We prove below the direct estimate for the modified operators which preserve $e_{r}$. Let $\pi_{r}$ denote the space of all algebraic polynomials of degree $r$ and suppose $C_{B}(0, \infty)$ is the space of all continuous and bounded functions on $(0, \infty)$ endowed with the norm $\|f\|=\sup \{|f(x)|: x \in(0, \infty)\}$. Further let us consider the following $K$-functional:

$$
K_{2}(f, \delta)=\inf _{g \in C_{B}^{2}(0, \infty)}\left\{\|f-g\|+\delta\left\|g^{\prime \prime}\right\|\right\}
$$

where $\delta>0$ and $C_{B}^{2}(0, \infty)=\left\{g \in C_{B}(0, \infty): g^{\prime}, g^{\prime \prime} \in C_{B}(0, \infty)\right\}$.

Theorem 2.1. Let $f \in C_{B}(0, \infty)$, then for $r \in \mathbb{N}$ we have

$$
\left|\widetilde{P}_{n, r}(f, x)-f(x)\right| \leq C \omega_{2}\left(f, \sqrt{\delta_{n, r}}\right)+\omega\left(f,\left|\frac{(n+1)}{\left((n+1)_{r}\right)^{1 / r}}-1\right| x\right),
$$

where $C$ is a positive constant and $\delta_{n, r}$ is given as

$$
\delta_{n, r}=\left[\frac{(n+1)(2 n+3)}{\left((n+1)_{r}\right)^{2 / r}}-\frac{4(n+1)}{\left((n+1)_{r}\right)^{1 / r}}+2\right] x^{2} .
$$

Proof. We introduce the auxiliary operators $P_{n, r}: C_{B}(0, \infty) \rightarrow C_{B}(0, \infty)$ as follows

$$
P_{n, r}(f, x)=\widetilde{P}_{n, r}(f, x)-f\left(\frac{(n+1) x}{\left((n+1)_{r}\right)^{1 / r}}\right)+f(x) .
$$

These are linear operators and preserve linear functions. As by Lemma 2.1 and the positivity of $\widetilde{P}_{n, r}(t, x)$, we have

$$
P_{n, r}(t, x)=\widetilde{P}_{n, r}(t, x)-\frac{(n+1) x}{\left((n+1)_{r}\right)^{1 / r}}+x=\frac{(n+1) x}{\left((n+1)_{r}\right)^{1 / r}}-\frac{(n+1) x}{\left((n+1)_{r}\right)^{1 / r}}+x=x .
$$

Let $g \in C_{B}^{2}(0, \infty)$ and $x, t \in(0, \infty)$. By Taylor's formula, we have

$$
g(t)=g(x)+(t-x) g^{\prime}(x)+\int_{x}^{t}(t-u) g^{\prime \prime}(u) d u
$$


Then using Lemma 2.2 and by positivity of $\widetilde{P}_{n, r}$, we have

$$
\begin{aligned}
\left|P_{n, r}(g, x)-g(x)\right|= & \left|P_{n, r}\left(\int_{x}^{t}(t-u) g^{\prime \prime}(u) d u, x\right)\right| \\
= & \mid \widetilde{P}_{n, r}\left(\int_{x}^{t}(t-u) g^{\prime \prime}(u) d u, x\right) \\
& -\int_{x}^{\frac{(n+1) x}{((n+1) r)^{1 / r}}}\left(\frac{(n+1) x}{\left((n+1)_{r}\right)^{1 / r}}-u\right) g^{\prime \prime}(u) d u \mid \\
\leq & {\left[T_{2}^{\widetilde{P}_{n, r}}(x)+\left(\frac{(n+1) x}{\left((n+1)_{r}\right)^{1 / r}}-x\right)^{2}\right]\left\|g^{\prime \prime}\right\| } \\
:= & \delta_{n, r}\left\|g^{\prime \prime}\right\| .
\end{aligned}
$$

Next, by (2.7) and from Lemma 2.1, we have

$$
\left|P_{n, r}(f, x)\right| \leq \widetilde{P}_{n, r}(1, x)\|f\|+2\|f\| \leq 3\|f\| .
$$

Using (2.7), (2.8) and (2.9), we have

$$
\begin{aligned}
\left|\widetilde{P}_{n, r}(f, x)-f(x)\right| \leq & \left|P_{n, r}(f-g, x)-(f-g)(x)\right|+\left|P_{n, r}(g, x)-g(x)\right| \\
& +\left|f\left(\frac{(n+1) x}{\left((n+1)_{r}\right)^{1 / r}}\right)-f(x)\right| \\
\leq & 4|| f-g \|+\delta_{n, r}|| g^{\prime \prime}||+\left|f\left(\frac{(n+1) x}{\left((n+1)_{r}\right)^{1 / r}}\right)-f(x)\right| \\
\leq & C\left\{\|f-g\|+\delta_{n, r}|| g^{\prime \prime}||\right\}+\omega\left(f,\left|\frac{(n+1) x}{\left((n+1)_{r}\right)^{1 / r}}-x\right|\right) .
\end{aligned}
$$

Finally, if we take the infimum over all $g \in C_{B}^{2}(0, \infty)$, and using the inequality due to Gonska [8], $K_{2}(f, \delta) \leq C \omega_{2}(f, \sqrt{\delta}), \delta>0$, we get at once the desired result.

Corollary 2.1. Let $f \in \pi_{1}+C_{B}(0, \infty)$. Then

$$
\left|\widetilde{P}_{n, 1}(f, x)-f(x)\right| \leq C \omega_{2}\left(f, \frac{x}{\sqrt{(n+1)}}\right),
$$

where $C$ is certain positive constant.

Let us consider

$$
B_{2}(0, \infty):=\left\{f: f \in C(0, \infty) \rightarrow \mathbb{R} \text { and }|f(x)| \leq C(f)\left(1+x^{2}\right)\right\},
$$

where $C(f)$ is a positive constant depending only on $f$ and we denote $C_{2}(0, \infty)=$ $C(0, \infty) \cap B_{2}(0, \infty)$, by $C_{2}^{*}(0, \infty)$, we denote the subspace of all $f \in C_{2}(0, \infty)$ for which $\lim _{x \rightarrow \infty} \frac{f(x)}{1+x^{2}}<\infty$. The weighted modulus of continuity $\Omega(f, \delta)$ defined on infinite 
interval $\mathbb{R}^{+}$(see [11]) is defined as

$$
\boldsymbol{\Omega}(f, \delta)=\sup _{|h|<\delta, x \in \mathbb{R}^{+}} \frac{|f(x+h)-f(x)|}{\left(1+h^{2}+x^{2}+h^{2} x^{2}\right)}, \quad \text { for each } f \in C_{2}(0, \infty) .
$$

We now estimate the following quantitative Voronovskaja-type asymptotic formula.

Theorem 2.2. Let $f^{\prime \prime} \in C_{2}^{*}(0, \infty)$ and $r \in \mathbb{N}$. Then for $x>0$, we have

$$
\begin{aligned}
& \mid \widetilde{P}_{n, r}(f, x)-f(x)-\left[\frac{(n+1)}{\left((n+1)_{r}\right)^{1 / r}}-1\right] x f^{\prime}(x) \\
& \quad-\left[\frac{(n+1)_{2}}{\left((n+1)_{r}\right)^{2 / r}}-2 \frac{(n+1)}{\left((n+1)_{r}\right)^{1 / r}}+1\right] \frac{x^{2}}{2} f^{\prime \prime}(x) \mid \\
& \leq C\left(1+x^{2}\right) \Omega\left(f^{\prime \prime}, n^{-1 / 2}\right)\left[T_{2}^{\widetilde{P}_{n, r}}(x)+n^{2} T_{6}^{\widetilde{P}_{n, r}}(x)\right],
\end{aligned}
$$

where $C$ is certain absolute constant.

Proof. By Taylor's expansion,

$$
f(t)=\sum_{i=0}^{2}(t-x)^{i} \frac{f^{(i)}(x)}{i !}+h(t, x)(t-x)^{2},
$$

where

$$
h(t, x):=\frac{f^{\prime \prime}(\eta)-f^{\prime \prime}(x)}{2}
$$

we have

$$
\begin{aligned}
\widetilde{P}_{n, r}(f, x)-f(x) & =\widetilde{P}_{n, r}((f(t)-f(x), x) \\
& =T_{1}^{\widetilde{P}_{n, r}}(x) f^{\prime}(x)+\frac{T_{2}^{\widetilde{P}_{n, r}}(x)}{2} f^{\prime \prime}(x)+\widetilde{P}_{n, r}\left(h(t, x)(t-x)^{2}, x\right),
\end{aligned}
$$

and $h$ is a continuous function which vanishes at 0 and $\eta$ lies between $x$ and $t$.

Proceeding along the lines of [1, Theorem 2], we have

$$
\widetilde{P}_{n, r}\left(|h(t, x)|(t-x)^{2}, x\right) \leq C\left(1+x^{2}\right) \Omega\left(f^{\prime \prime}, \delta\right)\left(T_{2}^{\widetilde{P}_{n, r}}(x)+\frac{1}{\delta^{4}} T_{6}^{\widetilde{P}_{n, r}}(x)\right) .
$$

Finally, using Lemma 2.2 and choosing $\delta=\frac{1}{\sqrt{n}}$, we get the desired result.

Corollary 2.2. Let $f^{\prime \prime} \in C_{2}^{*}(0, \infty)$, then for $x>0$, we have

$$
\begin{aligned}
& \left|\widetilde{P}_{n, 1}(f, x)-f(x)-\frac{x^{2}}{2(n+1)} f^{\prime \prime}(x)\right| \\
\leq & C\left(1+x^{2}\right)\left(\frac{x^{2}}{(n+1)}+\frac{n^{2} x^{6}}{(n+1)^{5}}\left(5 n^{2}+160 n+265\right)\right) \Omega\left(f^{\prime \prime}, n^{-1 / 2}\right),
\end{aligned}
$$

where $C$ is certain absolute constant. 


\section{Estimates for Polynomially Bounded Functions}

In this section, we are going to extend our point wise estimates for unbounded functions defined on $(0, \infty)$ and having polynomial growth of order greater than 2 . In recent years several authors in $[2,4,17]$ and [14] have studied such problems. We recall that in Section 4 , we used $\Omega(f ; \delta)$ appropriate for functions with polynomial growth of order at most 2. To overcome this R. Păltănea introduced in [14] the weighted modulus $\omega_{\varphi}(f ; h)$ defined as

$$
\omega_{\varphi}(f ; h)=\sup \left\{|f(x)-f(y)|: x \geq 0, y \geq 0,|x-y| \leq h \varphi\left(\frac{x+y}{2}\right)\right\}, \quad h \geq 0,
$$

where $\varphi(x)=\frac{\sqrt{x}}{1+x^{m}}, x \in(0, \infty), m \in \mathbb{N}, m \geq 2$. We consider here those functions, for which we have the property

$$
\lim _{h \rightarrow 0} \omega_{\varphi}(f ; h)=0 .
$$

It is easy to verify that this property is fulfilled for $f$ an algebraic polynomial of degree less than or equal to $m$. This follows from Theorem 2 in [14], which states that $\lim _{h \rightarrow 0} \omega_{\varphi}(f ; h)=0$ whenever the function $f \circ e_{2}$ is uniformly continuous on $(0,1]$ and the function $f \circ e_{v}, v=\frac{2}{2 m+1}$ is uniformly continuous on $[1, \infty)$, where $e_{v}(x)=x^{v}$, $x \geq 0$. Let us denote by $W_{\varphi}(0, \infty)$ the subspace of all real functions defined on $(0, \infty)$, for which the two conditions mentioned above hold true. In [17] we studied positive linear operators $L_{n}: E \rightarrow C(0, \infty)$, where $E$ is a subspace of $C(0, \infty)$, such that $C_{k}(0, \infty) \subset E$ with $k=\max \{m+3,6,2 m\}$ and

$C_{k}(0, \infty)=\left\{f \in C(0, \infty)\right.$ exists $M>0:|f(x)| \leq M\left(1+x^{k}\right)$ for all $\left.x>0\right\}, \quad k \in \mathbb{N}$.

One of main results of [17] which we are going to apply for $\widetilde{P}_{n, r}$ is the following quantitative estimate in terms of weighted modulus $\omega_{\varphi}(f ; h)$ (see [17, Theorem 2.2]).

Theorem 3.1 (Theorem A). Let $L_{n}: E \rightarrow C(0, \infty), C_{k}(0, \infty) \subset E, k=\max \{m+$ $3,6,2 m\}$ be sequence of linear positive operators, preserving the linear functions. If $f \in C^{2}(0, \infty) \cap E$ and $f^{\prime \prime} \in W_{\varphi}(0, \infty)$, then we have for $x \in(0, \infty)$ that

$$
\begin{aligned}
& \left|L_{n}(f, x)-f(x)-\frac{1}{2} f^{\prime \prime}(x) \mu_{n, 2}^{L}(x)\right| \\
\leq & \frac{1}{2}\left[\mu_{n, 2}^{L}(x)+\sqrt{2} \sqrt{L_{n}\left(\left[1+\left(x+\frac{|t-x|}{2}\right)^{m}\right]^{2} ; x\right)}\right] \omega_{\varphi}\left(f^{\prime \prime} ;\left(\frac{\mu_{n, 6}^{L}}{x}\right)^{1 / 2}\right) .
\end{aligned}
$$

Here $\mu_{n, k}^{L}(x)=L_{n}\left((t-x)^{k}, x\right)$ is the $k$-th order central moment of $L_{n}$.

We point out here that the statement in Theorem A can be extended for positive linear operators which don't preserve linear functions what we need in this case is to add the term $\mu_{n, 1}^{L}(x) f^{\prime}(x)$ in the left side of (3.1). As an application of Theorem A, we have the following result for modified Post-Widder operators. 
Theorem 3.2. Let $\widetilde{P}_{n, r}: E \rightarrow C(0, \infty), C_{k}(0, \infty) \subset E, k=\max \{m+3,6,2 m\}$, be the sequence of linear positive operators, preserving the test function $e_{r}$. If $f \in$ $C^{2}(0, \infty) \cap E$ and $f^{\prime \prime} \in W_{\varphi}(0, \infty)$, then we have for $x \in(0, \infty)$ that

$$
\begin{aligned}
& \mid \widetilde{P}_{n, r}(f, x)-f(x)-\left[\frac{(n+1)}{\left((n+1)_{r}\right)^{1 / r}}-1\right] x f^{\prime}(x) \\
& \quad-\frac{x^{2}}{2} f^{\prime \prime}(x)\left[\frac{(n+1)_{2}}{\left((n+1)_{r}\right)^{2 / r}}-2 \frac{(n+1)}{\left((n+1)_{r}\right)^{1 / r}}+1\right] \mid \\
& \leq \frac{1}{2}\left[\left(\frac{(n+1)_{2}}{\left((n+1)_{r}\right)^{2 / r}}-2 \frac{(n+1)}{\left((n+1)_{r}\right)^{1 / r}}+1\right) x^{2}\right. \\
& \left.\quad+\sqrt{2} \sqrt{\widetilde{P}_{n, r}\left(\left[1+\left(x+\frac{|t-x|}{2}\right)^{m}\right]^{2} ; x\right)}\right] \\
& \quad \times \omega_{\varphi}\left(f^{\prime \prime} ;\left(\left[\frac{(n+1)_{6}}{\left((n+1)_{r}\right)^{6 / r}}-6 \frac{(n+1)_{5}}{\left((n+1)_{r}\right)^{5 / r}}+15 \frac{(n+1)_{4}}{\left((n+1)_{r}\right)^{4 / r}}\right.\right.\right. \\
& \left.\left.\left.\quad-20 \frac{(n+1)_{3}}{\left((n+1)_{r}\right)^{3 / r}}+15 \frac{(n+1)_{2}}{\left((n+1)_{r}\right)^{2 / r}}-6 \frac{(n+1)}{\left((n+1)_{r}\right)^{1 / r}}+1\right] x^{5}\right)^{1 / 2}\right) .
\end{aligned}
$$

We observe that the argument of $\omega_{\varphi}\left(f^{\prime \prime} ; \delta\right)$ in above theorem is of order $\delta=O\left(n^{-3 / 2}\right)$, $n \rightarrow \infty$.

Corollary 3.1. Under the assumption of above theorem, if the operators preserve test function $e_{1}$, then we have

$$
\begin{aligned}
& \left|\widetilde{P}_{n, 1}(f, x)-f(x)-\frac{x^{2}}{2(n+1)} f^{\prime \prime}(x)\right| \\
& \leq \frac{1}{2}\left[\frac{x^{2}}{n+1}+\sqrt{2} \sqrt{\widetilde{P}_{n, 1}\left(\left[1+\left(x+\frac{|t-x|}{2}\right)^{m}\right]^{2} ; x\right)}\right] \\
& \times \omega_{\varphi}\left(f^{\prime \prime} ;\left(\left[\frac{(n+2)_{5}}{(n+1)^{5}}-6 \frac{(n+2)_{4}}{(n+1)^{4}}+15 \frac{(n+2)_{3}}{(n+1)^{3}}\right.\right.\right. \\
& \left.\left.\left.-20 \frac{(n+2)_{2}}{(n+1)^{2}}+15 \frac{(n+2)}{(n+1)}-5\right] x^{5}\right)^{1 / 2}\right) \text {. }
\end{aligned}
$$

We consider $M_{n, k}^{L}=L_{n}\left(|t-x|^{k}, x\right)$ as the $k$-th order absolute moments of operators $L_{n}$.

The next main result of [17], which we are going to apply for the operators $P_{n, r}$ is the following quantitative variant of Voronovskaja theorem (see [17, Theorem 2.3]).

Theorem 3.3 (Theorem B). Let $L_{n}: E \rightarrow C(0, \infty), C_{k}(0, \infty) \subset E, k=\max \{m+$ $3,4\}$, be sequence of linear positive operators, preserving the linear functions. If 
$f \in C^{2}(0, \infty) \cap E$ and $f^{\prime \prime} \in W_{\varphi}(0, \infty)$, then we have for $x \in(0, \infty)$ that

$$
\begin{aligned}
& \left|L_{n}(f, x)-f(x)-\frac{1}{2} f^{\prime \prime}(x) \mu_{n, 2}^{L}(x)\right| \\
\leq & \frac{1}{2}\left[\mu_{n, 2}^{L}(x)+\frac{\sqrt{2}}{\sqrt{x}} \mu_{n, 2}^{L}(x) C_{n, 2, m}(x)\right] \omega_{\varphi}\left(f^{\prime \prime} ; \sqrt{\frac{\mu_{n, 4}^{L}(x)}{\mu_{n, 2}^{L}(x)}}\right),
\end{aligned}
$$

where

$$
C_{n, 2, m}(x)=1+\frac{1}{M_{n, 3}^{L}(x)} \sum_{k=0}^{m}\left(\begin{array}{c}
m \\
k
\end{array}\right) x^{m-k} \frac{M_{n, k+3}^{L}(x)}{2^{k}} .
$$

We suppose for the operators $L_{n}$ that

$$
\frac{M_{n, k}^{L}}{M_{n, 3}^{L}}, \quad 4 \leq k \leq m,
$$

is a bounded ratio for fixed $x$ and $m$, when $n \rightarrow \infty$.

Using Cauchy-Schwarz inequality, we have

$$
\widetilde{P}_{n, r}\left(|t-x|^{k}, x\right) \leq \sqrt{\widetilde{P}_{n, r}\left((t-x)^{2 k}, x\right)}
$$

Applying Lemma 2.2, we have

$$
\widetilde{P}_{n, r}\left(|t-x|^{k}, x\right)=O\left(n^{-k / 2}\right), \quad n \rightarrow \infty,
$$

and so it is easy to observe that $C_{n, 2, m}(x)$ is a bounded term for fixed $x$ and $m$ when $n \rightarrow \infty$. Also as in Theorem A, we point out that the statement in Theorem B can be extended in a similar way for positive linear operators, which don't preserve linear functions. As an application of Theorem B we obtain the following quantitative asymptotic Voronovskaja theorem for $\widetilde{P}_{n, r}$.

Theorem 3.4. Let $\widetilde{P}_{n, r}: E \rightarrow C(0, \infty), C_{k}(0, \infty) \subset E, k=\max \{m+3,4\}$, be sequence of linear positive operators, preserving the test function $e_{r}$. If $f \in C^{2}(0, \infty) \cap$ $E$ and $f^{\prime \prime} \in W_{\varphi}(0, \infty)$, then we have for $x \in(0, \infty)$ that

$$
\begin{aligned}
& \left|\widetilde{P}_{n, r}(f, x)-f(x)-T_{1}^{\widetilde{P}_{n, r}}(x) f^{\prime}(x)-\frac{T_{2}^{\widetilde{P}_{n, r}}(x)}{2} f^{\prime \prime}(x)\right| \\
\leq & \frac{T_{2}^{\widetilde{P}_{n, r}}(x)}{2}\left[1+\frac{\sqrt{2}}{\sqrt{x}} C_{n, 2, m}(x)\right] \omega_{\varphi}\left(f^{\prime \prime} ; x \sqrt{\frac{T_{4}^{\widetilde{P}_{n, r}}(x)}{T_{2}^{\widetilde{P}_{n, r}}(x)}}\right),
\end{aligned}
$$

where

$$
C_{n, 2, m}(x)=1+\frac{1}{M_{n, 3}^{\widetilde{P}_{n, r}}(x)} \sum_{k=0}^{m}\left(\begin{array}{c}
m \\
k
\end{array}\right) x^{m-k} \frac{M_{n, k+3}^{\widetilde{P}_{n, r}}(x)}{2^{k}} .
$$


Corollary 3.2. If $f, f^{\prime \prime}$ satisfy the same conditions as in the assumption of Theorem 3.4 , then we have for $x \in(0, \infty)$ that

$$
\lim _{n \rightarrow \infty} n\left[\widetilde{P}_{n, r}(f, x)-f(x)\right]=x f^{\prime}(x)+\frac{x^{2}}{2} f^{\prime \prime}(x) .
$$

Corollary 3.3. Under the assumption of above Theorem 3.4, if the operators preserve test function $e_{1}$, then we have

$$
\begin{aligned}
& \left|\widetilde{P}_{n, 1}(f, x)-f(x)-\frac{x^{2}}{2(n+1)} f^{\prime \prime}(x)\right| \\
\leq & \frac{x^{2}}{2(n+1)}\left[1+\frac{\sqrt{2}}{\sqrt{x}} C_{n, 2, m}(x)\right] \omega_{\varphi}\left(f^{\prime \prime} ; x \frac{\sqrt{3(n+3)}}{n+1}\right) .
\end{aligned}
$$

Our next goal in this section is to obtain estimates in terms of $K$-functional for polynomially bounded functions. We recall some notations from [4].

If $m \in \mathbb{N}$ is fixed we consider the weight

$$
\rho(x)=\rho_{m}(x)=(1+x)^{-m}, \quad x \in I \equiv[0, \infty) .
$$

The polynomials weighted space associated to $\rho$ is defined by

$$
C_{\rho}(I)=\left\{f \in C(I):\|f\|_{\rho}<\infty\right\},
$$

where

$$
\|f\|_{\rho}=\sup _{x \geq 0} \rho(x)|f(x)|
$$

In [4] it was used

$$
\phi(x)=\sqrt{(1+a x)(b x+c)}, \quad a \in \mathbb{N}_{0}, b>0, c \geq 0 .
$$

Here we set $a=b=c=1$, i.e.,

$$
\phi(x)=1+x .
$$

For $\lambda \in[0,1], s=1,2$, and $f \in C_{\rho}(I)$, we consider the $K$-functional

$$
K_{s, \phi^{\lambda}}(f, t)_{\rho}=\inf \left\{\|f-g\|_{\rho}+t^{s}\left\|\phi^{\lambda s} \cdot g^{(s)}\right\|_{\rho}, g \in W_{s, \lambda}^{\infty}(\phi)\right\},
$$

where $\left.W_{s, \lambda}^{\infty}(\phi)\right\}$ consists of all functions $g \in C_{\rho}(0, \infty)$ such that $g^{(s-1)}$ is absolutely continuous on every finite closed subinterval of $(0, \infty)$ and $\left\|\phi^{\lambda s} \cdot g^{(s)}\right\|_{\rho}<\infty$.

One of the main results in [4] is Theorem 1 which we cite here as (see [4, page 1498]).

Theorem 3.5 (Theorem C). For a positive integer $m, \rho(x)=(1+x)^{-m}$ and $\phi(x)=$ $\sqrt{(1+a x)(b x+c)}, a \in \mathbb{N}_{0}, b>0, c \geq 0$, and for positive linear operator $L_{n}: C_{\rho}(I) \rightarrow$ $C(I)$, we suppose the following conditions:

(i) $L_{n}\left(e_{0}\right)=e_{0}$;

(ii) there exist a constant $C_{1}$ and a sequence $\left\{\alpha_{n}\right\}, \alpha_{n} \rightarrow 0, n \rightarrow \infty$, such that

$$
L_{n}\left((t-x)^{2}, x\right) \leq C_{1} \alpha_{n} \phi^{2}(x) ;
$$


(iii) there exists a constant $C_{2}=C_{2}(m)$ such that for each $m \in \mathbb{N}$

$$
L_{n}\left((1+t)^{m}\right) \leq C_{2}(1+x)^{m}, \quad x \geq 0 ;
$$

(iv) there exists a constant $C_{3}=C_{3}(m)$, such that for each $m \in \mathbb{N}$

$$
\rho(x) L_{n}\left((t-x)^{2} / \rho(t) ; x\right) \leq C_{3} \alpha_{n} \phi^{2}(x), \quad x \geq 0 .
$$

Then for $\lambda \in[0,1]$ there exists a constant $C_{4}=C_{4}(m, \lambda)$ such that for any $f \in C_{\rho}(I)$, $x \in I, n \in \mathbb{N}$, one has

$$
\rho(x)\left|f(x)-L_{n}(f ; x)\right| \leq C_{4} K_{1, \phi^{\lambda}}\left(f ; \sqrt{\alpha_{n}} \phi^{1-\lambda}(x)\right)_{\rho}, \quad x \geq 0,
$$

where $K_{1, \phi^{\lambda}}(f ; t)_{\rho}$ is defined in (3.6) for $s=1$.

If in addition $L_{n}\left(e_{1}\right)=e_{1}$, then there exists a constant $C_{5}=C_{5}(m, \lambda)$ such that

$$
\rho(x)\left|f(x)-L_{n}(f ; x)\right| \leq C_{5} K_{2, \phi^{\lambda}}\left(f ; \sqrt{\alpha_{n}} \phi^{1-\lambda}(x)\right)_{\rho},
$$

where $K_{2, \phi^{\lambda}}(f ; t)_{\rho}$ is the $K$-functional defined in (3.6) for $s=2$.

We apply Theorem $\mathrm{C}$ for the modified Post-Widder operators $\widetilde{P}_{n, r}$. The condition (i) is trivial. The condition (ii) follows from the representation of second central moment $T_{2}^{\widetilde{P}_{n, r}}(x)$ in Lemma 2.2, with $\alpha_{n}=\frac{1}{n}$. The condition (iii) follows from representation of $\widetilde{P}_{n, r}\left(t^{k} ; x\right)=\frac{(n+1)_{k}}{\left((n+1)_{r}\right)^{k / r}} x^{k}$ obtained in previous section. To verify condition (iv) we apply Cauchy-Schwarz inequality and, by Lemma 2.2,

$$
\begin{aligned}
(1+x)^{-m} \widetilde{P}_{n, r}\left((t-x)^{2}(1+t)^{m} ; x\right) & \leq(1+x)^{-m} \sqrt{\widetilde{P}_{n, r}\left((t-x)^{4} ; x\right)} \sqrt{\widetilde{P}_{n, r}\left((1+t)^{2 m} ; x\right)} \\
& \leq(1+x)^{-m} C(r) T_{2}^{\widetilde{P}_{n, r}}(x) \sqrt{C_{2}(m)(1+x)^{2 m}} \\
& \leq C(r) \sqrt{C_{2}(m)} T_{2}^{\widetilde{P}_{n, r}}(x) \\
& \leq C_{3} \alpha_{n} \phi^{2}(x), \quad x \geq 0,
\end{aligned}
$$

where we have used condition (iii) and representation of $T_{2}^{\widetilde{P}_{n, r}}$ in Lemma 2.2. Therefore, as a consequence from Theorem $\mathrm{C}$, we obtain the following.

Theorem 3.6. Let $\rho(x)=(1+x)^{-m}, m \in \mathbb{N}, f \in C_{\rho}(I), \phi(x)=1+x, r \in \mathbb{N}, r \geq 2$, $\alpha=\frac{1}{n}$. Then for $\lambda \in[0,1]$ we have

$$
\rho(x)\left|\widetilde{P}_{n, r}(f ; x)-f(x)\right| \leq C(m, \lambda) K_{1, \phi^{\lambda}}\left(f ; \sqrt{\alpha_{n}} \phi^{1-\lambda}(x)\right)_{\rho} .
$$

For $r=1$ we have

$$
\rho(x)\left|\widetilde{P}_{n, 1}(f ; x)-f(x)\right| \leq C(m, \lambda) K_{2, \phi^{\lambda}}\left(f ; \sqrt{\alpha_{n}} \phi^{1-\lambda}(x)\right)_{\rho} .
$$

Remark 3.1. It is known (see [5]) that the $K$-functional $K_{s, \phi^{\lambda}}(f ; t)_{\rho}$ is equivalent to Ditzian-Totik moduli $\omega_{\phi^{\lambda}}^{s}(f ; t)_{\rho}$. In the most important cases $\lambda=0$ (point-wise Becker-type estimate) and $\lambda=1$ (estimate in norm) we can formulate (3.9) and (3.10) in terms of $\omega_{\phi^{\lambda}}^{s}(f ; t)_{\rho}$. If we denote by $C_{\rho, \infty}$ the set of all continuous functions 
on $(0, \infty)$ such that $\rho(x) f(x)$ has finite limit as $x \rightarrow \infty$, then it can be proved that $\lim _{t \rightarrow 0^{+}} \omega_{\phi^{\lambda}}^{s}(f ; t)_{\rho}=0$ whenever $f \in C_{\rho, \infty}(0, \infty)$. For $\lambda=0$ we get from here estimates (1.3) and (2.3)-(2.6) with some constant $C$ independent of $f$ and $n$ in place of 2 .

\section{Linear Combinations of Modified Post-Widder Operators}

From Corollary 2.1 and Corollary 3.2 we see that bounded continuous functions $f \in C_{B}(0, \infty)$ and also polynomially bounded functions can be approximated by $\widetilde{P}_{n, r}$ with order of approximation not greater than $O\left(n^{-1}\right)$. To increase the order of approximation we consider the linear combinations $L_{n, r}$ of $\widetilde{P}_{n, r}$. For more general settings and recent results for approximation by positive linear operators we refer the readers to the monograph [9]. Following the idea from [9] we will consider the following linear combinations

$$
L_{n, r}=\sum_{i=0}^{r} \alpha_{i}(n) \widetilde{P}_{n_{i}, r}
$$

where $n_{i}, i=0,1, \ldots$, are different positive numbers, $r \in \mathbb{N}$. Determine $\alpha_{i}(n)$ such that $L_{n, r} p=p$ for all $p \in \pi_{r}$ the set of algebraic polynomials of degree less than or equal to $r$. This seems to be natural as the operators $\widetilde{P}_{n, r}$ don't preserve linear functions if $r \geq 2$. The requirement that each polynomial of degree at most $r$ should be reproduced leads to the linear system

$$
L_{n, r}\left(t^{k}, x\right)=x^{k}, \quad 0 \leq k \leq r .
$$

From the nice representation of the images of monomials obtained in Section 2, $\widetilde{P}_{n, r}\left(t^{m}, x\right)=\frac{(n+1)_{m}}{\left((n+1)_{r}\right)^{m / r}} x^{m}$ and $(4.1)$, we obtain the system

$$
\left\{\begin{array}{l}
\alpha_{0}(n)+\alpha_{1}(n)+\alpha_{2}(n)+\cdots+\alpha_{r}(n)=1, \\
\sum_{i=0}^{r} \alpha_{i}(n) \frac{\left(n_{i}+1\right)_{m}}{\left(\left(n_{i}+1\right)_{r}\right)^{m / r}}=1, \quad 1 \leq m \leq r .
\end{array}\right.
$$

We observe that if $m=r$ in (4.3), the last equation coincides with the first one. So to have a unique solution for the coefficients $\alpha_{i}(n)$, we may impose additional condition

$$
L_{n, r}\left(t^{r+1}, x\right)=x^{r+1} .
$$

Then the system (4.3) will have the form

$$
\left\{\begin{array}{l}
\alpha_{0}(n)+\alpha_{1}(n)+\alpha_{2}(n)+\cdots+\alpha_{r}(n)=1, \\
\sum_{i=0}^{r} \alpha_{i}(n) \frac{\left(n_{i}+1\right)_{m}}{\left(\left(n_{i}+1\right)_{r}\right)^{m / r}}=1, \quad 1 \leq m \leq r-1, \\
\sum_{i=0}^{r} \alpha_{i}(n) \frac{\left(n_{i}+1\right)_{r+1}}{\left(\left(n_{i}+1\right)_{r}\right)^{(r+1) / r}}=1 .
\end{array}\right.
$$

Then from (4.5), we observe that all the polynomials of degree up to $r+1$ will be preserved by the combinations $L_{n, r}$ from (4.1). To obtain the direct estimate for 
approximation by linear combinations $L_{n, r}$, one need two additional conditions

$$
\begin{gathered}
n=n_{0}<n_{1}<n_{2}<\cdots<n_{r} \leq A n, \quad A=A(r), \\
\sum_{i=0}^{r}\left|\alpha_{i}(n)\right| \leq C .
\end{gathered}
$$

The condition (4.6) guarantees that

$$
L_{n, r}\left(|t-x|^{r+2}, x\right)=O\left(n^{-(r+2) / 2}\right), \quad n \rightarrow \infty
$$

The fact that all polynomials of degree less or equal to $r+1$ are preserved allow us to consider approximating functions $f$ from much larger class than $C_{B}(0, \infty)$, namely we consider $f \in \pi_{r+1}+C_{B}(0, \infty)$, where $\pi_{r+1}$ is the set of all algebraic polynomials of degree $\leq r+1$. Then following the same method applied by the authors in [10, Theorem 4] we arrive at the proof of following theorem.

Theorem 4.1. Let $f \in \pi_{r+1}+C_{B}(0, \infty)$. Then for every $x \in(0, \infty)$ and for $C>0$, $n>r$ and if the coefficients $\alpha_{i}(n)$ and numbers $n_{i}, 0 \leq i \leq r$, satisfy the conditions (4.5), (4.6) and (4.7), we have

$$
\left|L_{n, r}(f, x)-f(x)\right| \leq C \omega_{r+2}\left(f, n^{-1 / 2}\right) .
$$

Corollary 4.1. If $f \in \pi_{r+1}+C_{B}(0, \infty)$ and $f^{(r+2)} \in C_{B}(0, \infty)$, then

$$
\left|L_{n, r}(f, x)-f(x)\right| \leq C n^{-(r+2) / 2}|| f^{(r+2)} \|_{C_{B}(0, \infty)} .
$$

Let us consider the case $r=1$. In this case, to determine the coefficients $\alpha_{0}(n)$ and $\alpha_{1}(n)$ from (4.5), we get

$$
\left\{\begin{array}{l}
\alpha_{0}(n)+\alpha_{1}(n)=1, \\
\alpha_{0}(n) \frac{\left(n_{0}+1\right)_{2}}{\left(n_{0}+1\right)^{2}}+\alpha_{0}(n) \frac{\left(n_{1}+1\right)_{2}}{\left(n_{1}+1\right)^{2}}=1 .
\end{array}\right.
$$

The solution of this system is

$$
\alpha_{0}(n)=-\frac{n_{0}+1}{n_{1}-n_{0}}, \quad \alpha_{1}(n)=\frac{n_{1}+1}{n_{1}-n_{0}} .
$$

Obviously $\alpha_{1}(n)>0, \alpha_{0}(n)<0$. According to the conditions (4.6), (4.7), we must be careful with the choice of $\alpha_{0}(n), \alpha_{1}(n)$. For example, if $n_{0}=n, n_{1}=2 n$, then (4.11) implies

$$
\alpha_{0}(n)=-1-\frac{1}{n}, \quad \alpha_{1}(n)=2+\frac{1}{n},
$$

and (4.7) is satisfied. But if $n_{0}=n$ and $n_{1}=n+1$, then $\alpha_{0}(n)=-(n+1)$, $\alpha_{1}(n)=n+2$ and (4.7) is not fulfilled although (4.6) is true.

We observe that linear combinations $L_{n, 1}$ preserve $e_{0}, e_{1}, e_{2}$. Therefore, if $\pi_{2}$ denotes the space of all algebraic polynomials of degree 2 , we may consider the approximating functions $f$ to be every $f \in \pi_{2}+C_{B}(0, \infty)$, which means that we consider $f=g+h$, where $g \in \pi_{2}$ is an arbitrary algebraic polynomial of degree less than or equal to 2 
and $g \in C_{B}(0, \infty)$ is an arbitrary bounded continuous function. With $\alpha_{0}, \alpha_{1}$ defined in (4.11) we have

Theorem 4.2. Let $f \in \pi_{2}+C_{B}(0, \infty)$. Then for every $C>0, n>1$, we have the following:

$$
\left|L_{n, 1}(f, x)-f(x)\right| \leq C \omega_{3}\left(f, n^{-1 / 2}\right) .
$$

If $f^{\prime \prime \prime} \in C_{B}(0, \infty)$, then

$$
\left|L_{n, 1}(f, x)-f(x)\right| \leq C n^{-3 / 2}|| f^{\prime \prime \prime}||_{C_{B}(0, \infty)} .
$$

Remark 4.1. We observe that the estimates with linear combinations $L_{n, r}$ from Theorem 4.1 and Corollary 4.1 are better than the previous estimates [16, (1.3)], also from [10]. The reason for this effect is that our modified Post-Widder operators $P_{n, r}$ preserve $e_{0}$ and $e_{r}$.

Acknowledgements. The authors are thankful to the reviewers for helpful remarks and suggestions which lead to essential improvement of the whole manuscript.

\section{REFERENCES}

[1] T. Acar, A. Aral and I. Rasa, The new forms of Voronovskaja's theorem in weighted spaces, Positivity 20 (2016), 25-40.

[2] A. Aral, H. Gonska, M. Heilmann and G. Tachev, Quantitative Voronovskaya-type results for polynomially bounded functions, Results Math. 70(3-4) (2016), 313-324.

[3] D. Aydin, A. Aral and G. B-Tunca, A generalization of Post-Widder operators based on qintegers, An. Stiint. Univ. Al. I. Cuza Iasi. Mat. (N.S.), DOI 10.2478/aicu-2014-0012.

[4] J. Bustamante, J. M. Quesada and L. M. de la Cruz, Direct estimate for positive linear operators in polynomial weighted spaces, J. Approx. Theory 162(8) (2010), 1495-1508.

[5] Z. Ditzian and V. Totik, Moduli of Smoothness, Springer-Verlag, New York, 1987.

[6] B. R. Draganov and K. G. Ivanov, A characterization of weighted approximation by the PostWidder and the Gamma operators, J. Approx. Theory 146 (2007), 3-27.

[7] B. R. Draganov and K. G. Ivanov, A characterization of weighted approximation by the Gamma and the Post-Widder operators, in: B. Bojanov, M. Drinov (Eds.), Proceedings of the International Conference on "Constructive Theory of Functions, Varna 2005", Academic Publishing House, Sofia, 2006, 80-87.

[8] H. Gonska, Quantitative Aussagen zur Approximation durch positive lineare Operatoren, $\mathrm{Ph}$. D. Thesis, Universität Duisburg, Duisburg, 1979.

[9] V. Gupta and G. Tachev, Approximation with Positive Linear Operators and Linear Combinations, Series: Developments in Mathematics 50, Springer, Cham, 2017.

[10] V. Gupta and G. Tachev, Approximation by Szász-Mirakyan Baskakov operators, J. Appl. Funct. Anal. 9(3-4) (2014), 308-319.

[11] N. Ispir, On modified Baskakov operatos on weighted spaces, Turk. J. Math. 25 (2001), 355-365.

[12] S. Li and R. T. Wang, The characterization of the derivatives for linear combinations of Post Widder operators in $L_{p}$, J. Approx. Theory 97 (1999), 240-255.

[13] C. P. May, Saturation and inverse theorems for combinations of a class of exponential-type operators, Canad. J. Math. 28 (1976), 1224-1250.

[14] R. Pǎltǎnea, Estimates of approximation in terms of a weighted modulus of continuity, Bull. Transilv. Univ. Brasov 4(53) (2011), 67-74. 
[15] R. K. S. Rathore and O. P. Singh, On convergence of derivatives of Post-Widder operators, Indian J. Pure Appl. Math. 11 (1980), 547-561.

[16] L. Rempulska and M. Skorupka, On Approximation by Post-Widder and Stancu operators Preserving $x^{2}$, Kyungpook Math. J. 49 (2009), 57-65.

[17] G. Tachev and V. Gupta, General form of Voronovskaja's theorem in terms of weighted modulus of continuity, Results Math. 69(3-4) (2016), 419-430.

[18] D. V. Widder, The Laplace Transform, Princeton Mathematical Series, Princeton University Press, Princeton, New Jersey, 1941.

${ }^{1}$ Department of Mathematics,

Netaji Subhas University of Technology,

Sector 3 Dwarka, New Delhi 110078, India

Email address: vijaygupta2001@hotmail.com

${ }^{2}$ UnIVERSITY OF ARChiteCtURE,

Civil Engineering and Geodesy,

1 Hristo Smirnenski, Blvd., Sofia-1046, Bulgaria

Email address: gtt_fte@uacg.bg 



\title{
KRAGUJEVAC JOURNAL OF MATHEMATICS
}

\begin{abstract}
About this Journal
The Kragujevac Journal of Mathematics (KJM) is an international journal devoted to research concerning all aspects of mathematics. The journal's policy is to motivate authors to publish original research that represents a significant contribution and is of broad interest to the fields of pure and applied mathematics. All published papers are reviewed and final versions are freely available online upon receipt. Volumes are compiled and published and hard copies are available for purchase. From 2018 the journal appears in one volume and four issues per annum: in March, June, September and December. From 2021 the journal appears in one volume and six issues per annum: in February, April, June, August, October and December.

During the period 1980-1999 (volumes 1-21) the journal appeared under the name Zbornik radova Prirodno-matematičkog fakulteta Kragujevac (Collection of Scientific Papers from the Faculty of Science, Kragujevac), after which two separate journalsthe Kragujevac Journal of Mathematics and the Kragujevac Journal of Science-were formed.
\end{abstract}

\section{Instructions for Authors}

The journal's acceptance criteria are originality, significance, and clarity of presentation. The submitted contributions must be written in English and be typeset in $\mathrm{T}_{\mathrm{EX}}$ or $\mathrm{AT}_{\mathrm{E} X} \mathrm{x}$ using the journal's defined style (please refer to the Information for Authors section of the journal's website http://kjm.pmf.kg.ac.rs). Papers should be submitted using the online system located on the journal's website by creating an account and following the submission instructions (the same account allows the paper's progress to be monitored). For additional information please contact the Editorial Board via e-mail (krag_j_math@kg.ac.rs). 